\title{
Mereological nihilism: quantum atomism and the impossibility of material constitution
}

\author{
Jeffrey Grupp
}

Received: 1 September 2005/ Accepted: 20 April 2006

(C) Springer Science+Business Media B.V. 2006

\begin{abstract}
Mereological nihilism is the philosophical position that there are no items that have parts. If there are no items with parts then the only items that exist are partless fundamental particles, such as the true atoms (also called philosophical atoms) theorized to exist by some ancient philosophers, some contemporary physicists, and some contemporary philosophers. With several novel arguments I show that mereological nihilism is the correct theory of reality. I will also discuss strong similarities that mereological nihilism has with empirical results in quantum physics. And I will discuss how mereological nihilism vindicates a few other theories, such as a very specific theory of philosophical atomism, which I will call quantum abstract atomism. I will show that mereological nihilism also is an interpretation of quantum mechanics that avoids the problems of other interpretations, such as the widely known, metaphysically generated, quantum paradoxes of quantum physics, which ironically are typically accepted as facts about reality. I will also show why it is very surprising that mereological nihilism is not a widely held theory, and not the premier theory in philosophy.
\end{abstract}

Keywords Mereology · Parts and wholes - Composition · Material constitution · Relations · Philosophy of physics · Atomism - Quantum theory · Levels of reality · Mereological nihilism · Particles · Democritus · Indian Buddhism · Quantum physics - Interpretations of quantum mechanics: material constitution ·

Wave-particle duality · Quantum uncertainty · Metaphysics · Relational properties · Strata $\cdot$ Immaterialism $\cdot$ Energy

\section{Introduction}

Mereological nihilism is the theory that the only things that exist are the atomic (partless) quantum particles. In other words, only partless fundamental particles

J. Grupp (ه)

Philosophy Department, Purdue University, West Lafayette 47907 IN, USA

e-mail: jeffgrupp@aol.com 
exist (electrons, quarks, etc.), they do not compose any composite objects, and thus empirical reality does not exist. Traditionally, and more specifically, mereological nihilism is the philosophical position that objects that have parts do not exist. ${ }^{1}$ If an item $\mathrm{C}$ is believed to exist due to the belief that it is composed of two parts, $\mathrm{a}$ and $\mathrm{b}$, $\mathrm{C}$ does not exist; it is a figment of the conceptual imagination, a mental fabrication. (Also, if a, for example, is believed to exist due to the belief that it is made of parts $\mathrm{p}$ and $\mathrm{q}$, then a also does not exist.)

In this article, I will show that there is compelling scientific evidence, and robust philosophical reasoning, which shows that mereological nihilism is the correct theory of reality. If it is, then the only objects that exist are quantum objects that do not have parts, such as electrons, quarks, and any other partless quantum objects. Hereafter, I will call these "quantum atoms," "quantum philosophical atoms," or "quantum abstract atoms," for reasons I will clarify. As I will discuss in this introductory section, the data of experimental quantum physics reveals that the partless quantum abstract atoms are point-sized (sizeless), unstructured, non-material, surfaceless, non-interacting, irreducible, and perhaps indistinguishable quantum objects (true philosophical atoms) that have an incredibly short-lived and/or nearly instantaneous existence. Philosophical reasoning will also be given later in this article to show that philosophical argumentation about the quantum realm is in agreement these experimental findings. These experimental findings of quantum physics show that quantum objects are not the sorts of items that can constitute macroscopic objects-or any objects whatsoever: material constitution is an illusion, and thus everyday ordinary empirical-material reality is some sort of a dream.

In this article, in discussing quantum philosophical atoms, I will use the description of them given in the previous paragraph since that is the way that quantum physicists have discovered the quantum philosophical atoms to be. This description will be clarified in detail in this section, and it is a description of the quantum philosophical atoms that is the strictly empirical, non-metaphysical account of quantum mechanics. In this article, I will explain how the word "empirical" will be used, and how there is consequently no conflict with how I argue for both of the following positions: quantum mechanics is empirical, mereological nihilism show the empirical does not exist. ${ }^{2}$

Quantum physics is an empirical science, but oddly, many quantum physicists have combined it with a metaphysical philosophy that involves all sorts of unobservable, non-empirical, non-scientific, non-experiential, and nonsensical items (e.g. collapsing probability waves, existent past times, smeared point-particles, waveparticle duality, where waves are unobservables, etc.). As I will discuss, these

\footnotetext{
${ }^{1}$ Mereological nihilism is also called compositional nihilism (Rosen and Dorr 2002)) or eliminativism (Merricks 2001). Some philosophers also consider mereological nihilism and monadism to be identical positions. This would be the case if "monadism" is defined as Hoffman and Rosenkrantz do in the following passage: "...monadism attacks the commonsense view on the ground that only true atoms or indivisibles exist... Monadism has the radical implication that there are no compound material objects, either living or non-living." (Hoffman and Rosenkrantz 1997, 77)

${ }^{2}$ What will be shown is that there is more than one way to use the word "empirical." There is the ordinary macroscopic empirical reality, which is a dream, and there is the quantum empirical reality, which is real. "Empirical" is usually used to refer to something to do with the macroscopic objects of immediate ordinary experience. I will however discuss, as others have, that the word "empirical" must have several meanings in addition to that one. And I will find that the ordinary empirical reality cannot exist, and only another sort of empirical experiencing that is associated with quantum science can be considered empirical experience that actually represents real items.
} 
metaphysical items can be shown to be impossible entities (i.e., non-existent entities) that have nothing to do with the strictly empirical quantum findings (i.e. the strictly scientific findings) of quantum physics. I will show that, for that reason, the metaphysical items only introduce unneeded and unjustified unintelligibility and contradiction into the quantum mechanics, and can even prohibit further investigation and/or understanding of quantum reality.

Most of the currently accepted interpretations of the data of quantum mechanics involve the idea that quantum reality involves paradox, but I will show that the mereological nihilist interpretation of quantum mechanics is non-paradoxical. I do this by showing that quantum reality is best describable as a mereological nihilist reality, and since mereological nihilism is non-paradoxical, then quantum reality is also non-paradoxical. In other words, the mereological nihilist account of reality is non-metaphysical and consistent, whereas the other interpretations of quantum physics (such as Copenhagen) are paradoxical and are diametrically opposed to the mereological nihilist interpretation. I will point out that this makes the nonmetaphysical mereological nihilistic interpretation of quantum mechanics more appealing than the other existing and popular interpretations of quantum reality, which involve extramental and extrasensory items. I will also point out how it is actually the ordinary macroscopic empirical realm of reality-which is only a conceptual realm - that is riddled with paradox and antinomy (e.g., the problem of change).

I will show that quantum physicists have often mistakenly asserted that quantum reality cannot be atomistic, and I will show that quantum reality is composed of point atoms. I will show that if quantum physics is merely purged of its metaphysical elements, and if "atomism" ceases to inappropriately be used to only denote ancient Greek atomism (since that is just one of the many atomism traditions), then an empirically based quantum atomism is verified which does not involve metaphysical elements nor paradox. I will show that because physicists have erroneously considered atomism to be only of one variety (Greek Democritean atomism), and because physicists have so often needlessly strayed from the strictly empirical findings of quantum mechanics in order to incorporate extrasensory, non-scientific, metaphysical theorization, it is for those two reasons that physicists have oddly not espoused empirical and logical theory of quantum atomism.

I will show that, in accord with the empirical data, the quantum point-atoms do not accumulate, connect, attach, touch, mix, adhere, gel, behave, or interrelate in any way to compose composite objects. Ubiquitously, it is demanded by scientists and philosophers that quantum reality partially or fully gives rise to macroscopic reality. But no theory exists to give us even the faintest hint of how it does. ${ }^{3}$ Quantum reality and macroscopic ordinary empirical reality utterly contradict one another, where the

\footnotetext{
${ }^{3}$ Some physicists may disagree with this, maintaining that, for example, metaphysical electron probability clouds give an explanation of how point-sized electrons give rise to smooth macro-like shapes in quantum reality, wherein these shaped items can just bunch up to accumulate into a universe. I however attack this in Sect. 6 below, finding that there cannot be any such metaphysical clouds and wavefunctions. The clouds are generated on computer screens, and do not represent the real nature of the indirectly observable electron, which whenever observed is only a point. Rather, the clouds are generated from many unconnected observations put together. Other than that, there are clear conceptual difficulties with maintaining that a point object can compose an extended surface, which, as mentioned in the next footnote, will also be attacked; and there are obvious problems with maintaining that an electron can be an extended object with a shape.
} 
construction of empirical reality out of quantum flashes of energy is analogous to maintaining that $0+0+0 \ldots$ could add up to one of the positive integers $[1,2,3,4, \ldots]^{4}$ It is only due to the non-empirical and metaphysical concepts that physicists and philosophers have filled quantum physics with, and due to misguided trust in ordinary empirical experience, that allow philosophers and physicists to unconvincingly attempt to assert that quantum reality composes a macroscopic reality. For these reasons, and for reasons I will discuss in detail in Sect. 6, mereological nihilism is an interpretation of quantum mechanics that is an antidote to the largely metaphysical interpretations we have been offered by physicists. ${ }^{5}$

This introduction is divided into several subsections where I will discuss introductory concepts to do with experimental quantum physics, and which are essential to understanding the quantum theory of mereological nihilism. In Sect. 2 of this article I introduce mereological nihilism in more detail, and my specific novel arguments that show that material constitution is impossible and that mereological nihilism is the best interpretation of quantum physics, are given in Sects. 3 and 4.

\subsection{Basic issues in conceptual quantum physics}

The rudimentary conceptual features of experimental quantum physics are not widely developed, nor widely agreed upon, by the quantum physicists. The goal of this article is to advance the conceptual understanding of quantum physics by showing that mereological nihilism appears to be the only possible interpretation of quantum mechanics that matches the empirical data, and thus the atomism of mereological nihilism offers the best conceptual understanding of quantum reality. In this first subsection I will discuss a few of the basic issues of conceptual quantum physics. Then in the rest of the introductory section I will introduce the mereological nihilist model of quantum physics.

\subsubsection{Quantum particles are unstructured and sizeless/point-sized}

The data of quantum physics show that quantum objects appear to be points (sizeless). (I will show in Sect. 4 of this article that philosophical reasoning also shows that there can only be point-particles in quantum reality.) Consider the passage about electrons from the physicist Watson from his recent book on quarks:

\footnotetext{
${ }^{4}$ Many philosophers will argue here that this is not an absurdity, since something like this was proven by Grünbaum (1952), when he proved that a continuum of points can compose an extended line. But I will show in Sect. 4.6 that his solution is incorrect, that extensions composed of points are impossible, and Zeno's Measure Paradox is no paradox at all.

${ }^{5}$ It will become apparent in Sect. 4 that mereological nihilism can only be anti-metaphysical. This will be become clear in that section, but it might be worthwhile to introduce these ideas here. According to mereological nihilism, it can be shown that no items contact or connect. This would include, for example, properties linking to particulars, relations relating relata, and so forth. If properties cannot link to particulars, then they are free-floating. This is to say that there are no properties that are instantiated, and thus reality cannot involve property instantiation. Thus, describing reality with property talk (e.g., $\mathrm{x}$ has $\mathrm{F}$, where $\mathrm{F}$ is a property) is impossible according to the mereological nihilist account. But virtually all contemporary metaphysics depends on property instantiation (see Grupp 2006a) and/or relational properties (see Grupp 2005b), and thus if mereological nihilism undercuts those, then there is no non-contradictory metaphysics. If what I have just written here is correct, then there is a clear connection between mereological nihilism and blob theory (which is the theory that $\mathrm{n}$-adic properties do not exist).
} 
"Electrons are accepted as truly fundamental particles, indivisible and having no measurable size, so far as anybody knows." "Watson goes on later to talk about other quantum particles as being points, such as neutrinos. ${ }^{7}$ There are a few reasons why this is the case, and one is that many quantum objects appear to not have any parts. It is however not entirely clear from what physicists have shown us that electrons, neutrinos, and other partless quantum objects are points just because nobody has found any parts that they might have. I will however present a new argument in Sect. 4.2 that shows why any quantum philosophical atom can only be a point (i.e. any partless quantum object can only be a point).

Physicists often call partless quantum objects unstructured objects. An object is unstructured if it does not have parts or size, just as an electron or neutrino is apparently unstructured. This is the common way physicists refer to the quantum particles that do not have parts or size, as show in the following passage by the famous physicist Gordon Kane:

Why... do we [physicists] think that electrons and quarks are the true 'Greek atoms'...? [I]nvestigators have tried by many means to determine whether electrons, quarks... and gluons show any evidence of structure, and they have not found any. These experiments probed perhaps 10,000 times further than it took to see structure in the past, but electrons and quarks continue to behave as point-like objects with no parts. (Kane 2000, 21)

\subsubsection{Quantum particles are indistinguishable and "not separate" from one another}

Indistinguishability between quantum objects (in particular the quantum atoms, since they are the only quantum objects that exist) means there are often no differences that can be pointed out between particles that are at different places. $^{8}$ In other words, quantum philosophical atoms are indistinguishable particles that are not coinciding (not overlapping): quantum atoms share all the same perceived properties, and thus from what is observed about them through quantum instrumentation, those observations do not provide data that allows quantum researchers to clearly distinguish quantum abstract atoms from one another. ${ }^{9}$ Along the same lines, the evidence for mereological nihilism also leads to the thesis that quantum philosophical atoms are indistinguishable: mereological nihilism is the theory that reality does not involve any parts and wholes, and if it does not, then only one thing exists. For that reason, two principle points can be made about mereological nihilism and the particles observed in quantum experiential physics:

\footnotetext{
${ }^{6}$ Watson $(2004,5)$.

7 Ibid., p. 7.

8 I will point out below why what might be believed to be differences of location do not constitute genuine differences between quantum particles.

9 I use the phrase "perceived properties" deliberately here, since properties of quantum particles do not appear to be actual properties of the particles themselves, but rather they appear to be events caused in the consciousness of the observer. On this account, a property of a quantum object, such as charge, is an idea rather than a real mind-dependent property. I will show below why mereological nihilism leads to the thesis that all properties are of this nature-they are concepts, not real entities out in the world separate from consciousness.
} 
1. The particles that exist can only be philosophical atoms (for example, electrons exist, and protons do not), and

2. The particles that exist must be indistinguishable.

Empirically based quantum physics does not necessarily lead to the position that all quantum atoms are indistinguishable, but it comes quite close to such a position for a number of reasons. The famous physicists, Brown and Davies, write: "All members of a given particle species are identical; there is on way to distinguish, say, one electron from another." 10 This passage appears to be in violation with mereological nihilism since it implies that reality can involve distinctions-in this case, distinctions between electrons and quarks, for example (but not between electrons and electrons, or quarks and quarks). But it is likely that such supposed distinctions between the electrons and the quarks, for example, will be wiped out with further research in the future in physics and philosophy. I discuss several reasons for this in later sections, but it is worth making a few points here about this issue.

Quantum non-separability (also called quantum non-locality) involves the discovery that analysis and manipulation of a particle here affects a particle somewhere else (perhaps even trillions of miles away) instantly, since the two particles are in some sense not separate. Quantum non-separability was first confirmed in the lab by the French physicist Alain Aspect, and it shows how particles that seem to be distinct since they appear to be at different locations, in fact are not distinct. Quantum nonseparability was resisted for decades by physicists, but with repeatable laboratory confirmation it has moved toward becoming a mainstream quantum thesis. ${ }^{11}$ Quantum non-separability gives more reason to conjecture that physics will continue to move from the idea that particles are separate and distinct, to the idea where particles are found to be indistinguishable and not separate. Non-local and entangled (non-separate) quantum atoms are not individualistic form one to the next. The discovery that quantum atoms are non-local indicates that they exhibit inseparability (or non-locality, which is also called quantum wholeness, or quantum entanglement), and it may be the case that all particles exhibit quantum inseparability. ${ }^{12}$ This quantum finding does away with the strict individuality of all particles.

\subsubsection{Quantum particles are unconnected}

Quantum abstract atoms do not stand in any relations to one another, and they are unconnected and unattached to one another. Contrary to popular belief, forces and quantum fields are not continuous (unbroken) connections between quantum objects. These issues will be discussed and clarified upon to a great extent in this article, since they are the essence of mereological nihilism.

There are many reasons why the erroneous idea that there are relations and continuous connections between quantum atoms have arisen. For example, metaphysicians might suggest that if quantum particles are entangled and exhibit quantum inseparability, it may seem to follow that they are connected or interrelated, in

\footnotetext{
${ }^{10}$ Davies and Brown $(1992,21)$.

${ }^{11}$ Even with the empirical confirmation, discussion of quantum non-separability is for some reason resisted or rejected by some relativity theorists.

12 Nadeau and Kafatos (1999, 4).

望 Springer
} 
some sense, and it may seem that reference to these indistinguishable quantum philosophical atoms also involves the reference to metaphysical connections or relations-namely the relations indistinguishable and not collocated, that stand between the quantum particles. Another way to put the issue is as follows: if particle $\mathrm{A}$ that is here, and particle $\mathrm{B}$ that is there, where $\mathrm{A}$ and $\mathrm{B}$ are one light year apart, it might seem that they exist in a relationship to one another, which could be called, at a distance of one light year apart. As just eluded to, I will show with multiple arguments, and with the quantum scientific data, in sections below that there are no relations or connections of any sort between any objects, ${ }^{13}$ what are ordinarily believed to be extramental connections or relations between objects are merely mental-imaginary concepts, and no such metaphysical items exist whatsoever outside of consciousness. The reasoning in that section will show that relations can only be concepts, and merely the products of the ways that humans organize their experiences about empirical reality. I have argued this point elsewhere $^{14}$ where I have shown that reality is utterly relationless and connectionless. Here is a passage of mine from another article where I showed that a reality involving metaphysical relations is merely a construction of consciousness, not a discovery of what reality is like:

Perhaps humans, in experiencing physical objects, have certain experiences of the objects, such as seeing $\mathrm{p}_{1}$ at $\mathrm{x}$, and seeing $\mathrm{p}_{2}$ at $\mathrm{y}$, and in comparing them mentally, invent concepts, such as that there are real, mind-independent relations in nature (such as many of the relations I have discussed in this paper: taller than, brotherhood, distance, and so on) between entities, where we imagine a real connection between them, and we do not recognize that we might only behold just the non-collocated spatial objects. In other words, it is arguable that what is experienced are two objects, $\mathrm{p}_{1}$ and $\mathrm{p}_{2}$, and the mind adds on, from concepts to do with spatial issues (such as size and location), ideas of relations or relatedness between spatially separated objects. It may be the case that a perceiver experiences objects $\mathrm{p}_{1}$ at $\mathrm{x}$ and $\mathrm{p}_{2}$ at $\mathrm{y}$, but only in the mind is there any sort of connection between them. For these reasons, the sentence, "the elephant is taller than the lion", need not be false, for it corresponds to three specific entities: the statement describes three experiences: (i) the experience of a lion, (ii) the experience of a elephant, and (iii) the experience of comparing the of (i) and (ii), where, unlike the experiences of (i) and (ii), the item denoted by "taller than" is a concept that does not represent anything outside of that experience. (Each of (i)-(iii) are experiences a perceiver has, but (iii), unlike (i) and (ii), is an experience of something not in the world outside the mind. ${ }^{15}$

Notice that reasoning such as this would be reasoning against other philosophies that are often considered similar to mereological nihilism, such as Quinnean nominalism, or the supposed nihilism of Trenton Merricks-both of which involve relations between objects (the relation set membership for the former, and the relation arrangement for the latter).

\footnotetext{
${ }^{13}$ I also argued this point in Grupp (2005b, d, 2006a, b), but the arguments I present in this article are quite different than those, and there is no redundancy between those articles and this one.

${ }^{14}$ Such as Grupp (2005b).

15 Grupp (2005b, 121-122).
} 


\subsubsection{The only quantum particles that exist are philosophical atoms}

Atomism traditionally has been very often oriented toward nihilism, as was the case in ancient Greece and ancient India, and often involved the rejection of the idea that there are metaphysical connections or relations between philosophical atoms. Atomism is a philosophy that goes back to the ancient philosophers, and some accounts of it-especially some accounts of the Buddhism of pre-classical India-appear to be surprisingly consistent with contemporary experimental quantum physics, and with the mereological interpretation of quantum physics that I will present in this article.

Instead of using the term "philosophical atoms," now the terms "simples," "physical simples," "mereological simples," or "material simples" are being extensively used. (In this introduction I will discuss why I do not use any of the terms just listed, and why I will instead use quantum abstract atoms to denote the partless fundamental particles.) I am not aware of any article that specifically points out why there has been this change from using the term "atoms" to "simples", but it seems we can find out what it's all about if we look at the philosophy of simples in the recent literature and compare it to atomism in the history of philosophy. It seems that the difference is that, unlike the philosophy of simples, the older theory, atomism, is more in accord with mereological nihilism. Schaffer writes that "the atomist claims that there are no macro-entities at all but only fundamental entities in various arrangements." ${ }^{, 16,17}$ But the philosophy of simples involves both basic building blocks and (at least some) wholes, and thus involves either a semi-nihilistic reality (i.e., only some of the wholes that humans ordinarily perceive actually exist) or an anti-nihilistic reality (i.e., there are as many wholes as humans ordinarily believe there are). In this article, for these reasons, I will use the term "atom", and I will not use "simple". Interestingly, the quasi-nihilism of philosophers such as Merricks and van Inwagen (both use "simples," and it seems van Inwagen got the whole trend of using "simples" going) are not thoroughgoing mereological nihilisms, since each of their nihilisms involve some composites, and since each of their nihilisms are not attempts to deny common sense and ordinary language. In this article, which can be considered a pure and quantum nihilism, no objects but partless quantum items are admitted, and an explicit of goal of this article is the rejection of ordinary language, where except in very rare circumstances, ordinary language can only be representative of illusions that the mind generates, not to reality itself.

The only medium I can use for communicating and describing mereological nihilism is language. For that reason, if I am to write an article on mereological nihilism, I have to write it from the non-nihilistic perspective since language is not nihilistic. Even though language is not found to be applicable to what really exists, as the reasoning of this article shows, if efforts are made to use language to discuss the partless atoms, then language can, in that case, come close to denoting real items, rather than illusory mental concepts. This does involve trickiness, however. For

\footnotetext{
16 Schaffer $(2003,498)$.

17 As I discuss elsewhere in this article, it is not entirely clear why a particle arrangement is not able to give rise to a composite entity: why isn't a topological network a mereological whole? Only if it can be shown, as I did elsewhere (Grupp 2005b, 2006a), that reality cannot involve any relations of arrangement is there no possibility of a composite entity, thus vindicating mereological nihilism as the correct theory.
} 
example, even though quantum philosophical atoms can only be indistinguishable in quantum mereological nihilism, in this article I however can do little more than discuss indistinguishable atoms as if they are distinct, such as when I write, for example, "atom $A_{1}$ and atom $A_{2}$ do not coincide." This sentence makes it appear that $\mathrm{A}_{1}$ and $\mathrm{A}_{2}$ are not indistinguishable, when in fact they are. This is an example of the difficultly of using ordinary language-which is composite-object-oriented, not a quantum oriented language-to discuss mereological nihilism.

\subsubsection{Quantum particles are immaterial}

I will discuss at great length in Sect. 2 of this article why quantum philosophical atoms are immaterial, rather than physical or material. There has been a lot of discussion among philosophers about non-physical or immaterial items. For example, holes have been suggested to be immaterial, since they are "made of nothing." This is just one of the many formulations that metaphysicians have given when discussing non-physical or immaterial items. In this article I will not focus on any of those metaphysical discussions, and I will only consider the concept of immateriality from one angle: an entity is immaterial if it does not have any of the features that an ordinary macroscopic object has. For example, if a macroscopic object is believed to have extension and be located in space, then an immaterial object is spatially unextended and spatially unlocated.

If quantum particles are immaterial, then that would indicate that all of reality is immaterial, since, as the quantum mereological nihilist argues, only immaterial particle-atoms exist. As quantum physicists have probed deeper and deeper into matter, they have discovered that matter does not exist, since literally nothing materialistic exists in quantum reality. The history of quantum physics can be looked at as, to some degree, quantum physicists attempting to describe to other people (who are not quantum scientists) a reality they cannot understand since their minds are attuned to the illusory and the materialistic, whereas the quantum reality is immaterialistic. Regarding this move toward the immaterialization of "matter" at the quantum level, Zimmerman writes: "The 19th and 20th centuries... witnessed the gradual 'dematerialization of matter'."18 George J. Stack also discusses this "dematerilzation" in a passage I give next. Stack's passage is quite significant since it is from the "Materialism" entry of the Routledge Encyclopedia of Philosophy. ${ }^{19}$ A passage about the immaterialism of quantum reality in such a widely read Encyclopedia is apparent evidence for how mainstream the idea is that there is no "matter" at the quantum level.

The twentieth-century conception of matter as composed of electrons, protons and other subatomic particles has spawned a rich speculative literature that effectively undermines previous forms of materialism. What the late US philosopher of science, Norwood Hanson, called the 'dematerialization' of matter, raises questions concerning what 'materialism' means in terms of the theories of microphysics. Many of the arguments that sustained earlier forms of materialism (including the assumption of causality as universal in nature) have been put in question.

\footnotetext{
18 Zimmerman (1996a, 166-167).

19 Edward Craig (ed.). 1998, New York: Routledge.
} 


\title{
1.1.6 Quantum particles do not touch each other or interact with one another
}

Even though philosophers and physicists often casually refer to "particle interaction" when they discuss what goes on in quantum reality (as if that means touching or contacting between quantum particles occurs), philosophers and physicists however have long maintained that interaction between particles cannot occur by contact or touching between particles. (This is one of the many ways that physicists and philosophers of physics have misled the public when they have attempted to explain the details of the immaterial quantum domain to ordinary people, who are oriented to think in terms of the macroscopic.) For example, consider the passage from Kline and Matheson that is from an article called "The Logical Impossibility of Collision":

\begin{abstract}
Absolutely no one still believes that every physical interaction consists of material bodies bumping into each other. Those who have tried to work out a completely mechanistic physics have been unable to explain common phenomena like liquidity, gravitation and magnetism. ${ }^{20}$
\end{abstract}

They go on to explain that particles interact via interrelating, or by mediating fields or forces. But I will show in this article that relations, fields, and forces do not exist, and I will show that the data from experimental quantum physics has never supported the idea that they do. I will also discuss many reasons why touching and contact between quantum particles is impossible.

Hoffman and Rosenkrantz, in their book Substance, also discuss this issue, and how it might lead to skepticism about the existence of parts and wholes (i.e., as evidence for mereological nihilism):

Skepticism about the intelligibility of the relations which are commonly thought to unify or organize the parts of material objects... might be thought to provide... argument in favor of monadism. ${ }^{21}$ Such skepticism or claims of ignorance might be fueled by developments in modern science, developments which are themselves well confirmed by experimental data. For example, the laws of physics imply that in all but extremely exceptional circumstances, no two physical objects touch (strictly speaking) because of the presence of repulsive forces between fundamental particles. ${ }^{22}$

Hoffman and Rosenkrantz do not indicate which "exceptional circumstances" they are referring to when particles touch. I will show below through textual evidence from physicists, and through philosophical reasoning, that quantum particles never touch, and thus the claim in the last sentence of Hoffman and Rosenkrantz is incorrect.

\subsubsection{Real quantum particles are philosophical atoms}

It is widely held by physicists that quantum particles are not true philosophical atoms. In this article I will show that physicists have not given evidence for why this

\footnotetext{
${ }^{20}$ Kline and Matheson $(1987,509)$. See Sect. 4 below for more discussion on this issue. van Inwagen discusses this $(1990,34)$.

${ }^{21}$ Hoffman and Rosenkrantz are using "monadism" here as synonymous with "mereological nihilism," see p. 77 of their 1997.

${ }^{22}$ Hoffman and Rosenkrantz $(1997,78)$.

Springer
} 
is the case, and I will show that such a position is incorrect. The word "atom", as I am using it here, does not refer to the atoms that scientists discuss (which are believed to be composed of one or more electrons and a nucleus), but rather to the philosophy of atomism: the philosophical position that what reality is, is no more than innumerable true atoms (partless atoms). Simons writes:

An atom is an individual with no proper parts, it is accordingly indivisible either in fact or in theory, as befits the etymology of its name. Atoms in this strict sense are not to be confused with atoms in the sense of physics and chemistry, which may have numerous proper parts and are far from indivisible, even in fact. Here the etymology of the name has lost touch with progress in physics; 'atom' is no longer a functional but a rigid term for certain natural kinds. Whether there really are mereological atoms is an unresolved question, and it is at present difficult to see what could resolve it. ${ }^{23}$

Many philosophers and physicists use the words "atom" or "atomism" as if they only refer to one theory: ancient Greek atomism (which carried over to later thinkers, such as Newton). This incorrect perspective has led many physicists to mistakenly believe that quantum reality cannot be an atomistic reality: physicists have imagined that atomist theories are only Greek/Democritean theories, and since quantum reality is not Greek/Democritean then quantum reality is not atomistic either. ${ }^{24}$ Greek/Democritean atomism involves the idea that philosophical atoms are like little irreducible solid objects, as if they are very similar to macroscopic objects except only much smaller-which is an idea diametrically opposed to quantum findings. In other words, the Greek/Democritean theory of atomism that goes back over 2,000 years to some of the ancient Greek philosophers is entirely outdated by the quantum discoveries that I will discuss in this article. I will show that the history of philosophy is in stark disagreement with the idea that there is only the Greek/ Democritean variety of atomism, as quantum physicists for some reason typically assume; atomism has had many varieties other than only the ancient Greek version. The primary non-Greek atomistic theory I will discuss is found in some versions of pre-classical Indian Buddhist atomism, which is in astonishingly close agreement with the quantum nihilistic atomism described in this article.

In discussing ancient Greek atomism, a few philosophers have attempted to maintain that a philosophical atom somehow can have parts, in some sense, such as Hoffman and Rosenkrantz, in a passage where they discuss voluminous material atoms (the so-called Democritean atoms, which have no similar characteristics to the quantum abstract atoms I argue for in this article):

...atoms are voluminous and (hence) spatially extended... First, by an atom we mean a material substance which has volume and which is necessarily indivisible. Second, because an atom is voluminous, it has parts. Thus what we maintain is that the parts of an atom are not substances. ${ }^{25}$

\footnotetext{
23 Simons $(1987,16)$.

24 This is just one reason that physicists have referred to their quantum physics as non-atomistic. They have added into their quantum physics all sorts of metaphysical items, as mentioned above, such as probability waves, which can be shown to be erroneous ideas, and the implanting of these metaphysical items into the interpretations of the quantum data are also reasons why physicists have espoused anti-atomism.
}

25 Hoffman and Rosenkrantz (1997, 51). 
I find this passage to be confusing, if not paradoxical. How can an atom be a substance made of non-substances? How can an atom be made of anything else? How can a philosophical atom have parts? It seems that Hoffman and Rosenkrantz merely present an ad hoc thesis by referring to the extended philosophical atom as a substance, and to refer to its "parts" as non-substances. The parts must also be extended, so why would they not also be substances, in the way that Hoffman and Rosenkrantz are using the word "substance"? Hoffman's and Rosenkrantz's account is in opposition to some, if not the majority, of the traditional accounts of atomism and ways of using the word "atomism" though history, wherein that traditional usage an atom is held to be partless and indivisible. Hoffman's and Rosenkrantz's ad hoc account appears to be an attempt to describe Democritean (extended) atoms in a way that covers-up the inconsistent ideas surrounding them (e.g., an atom has size, extension, but no parts ${ }^{26}$ ). Their passage shows how I will not use the word "atom" (or other synonymous terms: "philosophical atom," "quantum atom," or "quantum abstract atom") in this article. I use the word "atom" to only refer to partless items that do not have a spatial size, and I will attack Hoffman and Rosenkrantz's conception of the Democritean atom-in addition to all other accounts of the Democritean atom-in Sect. 4 below.

Quantum mereological nihilism can only be considered a non-Greek, nonDemocritean theory of philosophical atomism: an empirically based, scientifically aligned, anti-metaphysical, anti-realist atomism, where the quantum philosophical atoms are immaterial, non-solid, partless, inseparable (non-local), flashing points of immaterial energy (all these concepts will be discussed in detail in later sections). A shorter description of such particle might be, for example, "point particle," "unstructured particle," or, for example, "point charge" (these are all terms used by physicist, and point charge is the term physicists use to describe the electron). Since the dawn of the quantum revolution, it has become commonplace to find quantum physicists rejecting the idea that any quantum objects are philosophical atoms: "Quantum mechanics seems to contradict atomism."27 But it is hard to imagine how the unstructured (partless) point particles that physicists have discovered, such as electrons, quarks, etc., are not true philosophical atoms. (I specifically show with a novel argument in Sect. 4 that there cannot be point-sized objects that have parts.) There is no evidence that these items have parts, and there is no evidence that they have size, so how can't it be the case that the leading thesis for what these are, are true atoms? We can't cut electrons due to the fact that they are points and thus have no surface that we can plunge a knife into. If an electron, for example, did have parts, those parts would also be points, and thus would be, it seems, identical to the whole.

\subsubsection{Quantum particles are momentary and do not exhibit much motion}

The physicist, Ford, discusses the unfathomably temporary (momentary) existence of quantum atoms:

For a particle,... a hundredth of a second is an eternity... A gluon (the "glue" particle within a nucleus) lasts about that long between its creation and

\footnotetext{
${ }^{26}$ This is one of many inconsistencies I will point out in this article.

${ }^{27}$ Gibbins $(1987,2)$. Gibbins does not support this with evidence after making this assertion.

笔 Springer
} 
annihilation. A pion (a particle created in nuclear collisions) that moves a whole foot has traveled nearly a million billion times the diameter of a proton and has taken a lengthy $10^{-9}$ second to do it. Particles that live long enough to leave tracks in a detector have lifetimes of $10^{-10}$ to $10^{-6} \mathrm{~s}$. The neutron is a strange special case. With an average lifetime of fifteen minutes, it is the Methuselah of the particle world. ${ }^{28}$

Also, consider another passage from the physicist Paul Davies.

Until recently it was believed that electrons, protons, photons, and neutrinos are absolutely stable, i.e., that they have an infinite lifetime. The neutron can remain stable when trapped in a nucleus, but a free neutron decays in about $15 \mathrm{~min}$. All other known particles are highly unstable, their lifetimes varying from microseconds down to $10^{-23} \mathrm{~s}$. If these times seem incomprehensibly short, remember that a particle traveling at near the speed of light (which most accelerator products do) can cover $300 \mathrm{~m}$ in a microsecond. Particles that decay do so by the action of quantum processes, and so there is always an element of unpredictability. ${ }^{29}$

(Neutrons do not exist according to the mereological nihilist. The quarks that are believed to make up the bound neutron are never actually observed through the quantum machinery in the particle lab; they are only observed when they are free particles, wherein which they are momentary. The bound neutron is perceived, via the quantum machinery, to exist for minutes, rather than microseconds or shorter, and from that it is typically inferred that the quarks that allegedly compose a neutron are not momentary. This is, however, a metaphysical claim, since the quarks that allegedly compose the bound neutron are non-observables in these circumstances, and thus cannot be inferred to be non-momentary. Composite objects of any sort are perceived to be non-momentary, and quantum atoms are perceived through the quantum apparatuses in the lab to be momentary. Therefore, it is not surprising that neutrons are believed to be existent items that are non-momentary. But just as we cannot know anything about the nature of the quantum domain from analysis of composite bodies - such as a universe, a house, a bug, a chromosome, or a virus-we cannot infer from a non-momentary neutron that it is made of quarks that are non-momentary. For these reasons, I will not entertain the idea in this article that the quarks that are thought to be the constituents of bound neutrons are non-momentary, since no observations exist to support this conclusion.)

Quantum atoms are so short-lived that they cannot exhibit significant motion in their lifetimes. A macroscopic object, such as a pen, may travel with me from Purdue to UCLA and back, and then continue to travel with me all over West Lafayette wherever I go, perhaps for years. But quantum atoms are, conversely, unable to move in any such way, and comparably they are hardly moveable at all. Quantum atoms are momentary and do not exhibit much motion, which is quite the opposite of any sorts of objects of the macroscopic empirical level of perception. Even a cloud that is perceived to form and evaporate in $5 \mathrm{~min}$ is perceived to exist through an infinitude of duration as compared to any quantum atom. Combining these experimental findings about the momentariness of quantum objects with what has been

\footnotetext{
${ }^{28}$ Ford $(2004,15-16)$.

${ }^{29}$ Davies $(1984,82)$.
} 
described in the previous subsections above, it appears that perhaps the best way to describe a quantum atom would be to refer to it as a momentary flash of immaterial energy. The physicist Nick Herbert writes:

In the ... world of the quantum, a particle can vanish without a trace (quantum annihilation), or come into existence out of nowhere (quantum creation), move from location A to location B without being in between (quantum tunneling), or instantly flip from one state of being to another (quantum jumping). ${ }^{30}$

\begin{abstract}
All quantum measurements when scrutinized at their finest level of resolution consists of tiny particlelike events called "quanta", or "quantum jumps"flashes of light on a phosphor screen, for instance; or a bubble, spark, or click in a particle detector; the blackening of a silver grain in a photographic emulsion; or the sudden excitation of a light-sensitive molecule in your eye. The world when looked at closely appears to be made of little dots, much like color photos in a magazine. The first law of quantum theory is that these quantum jumps occur completely at random-no theory, quantum or otherwise, can predict where or when the next light-induced flash will occur in your retina. ${ }^{31}$
\end{abstract}

The overall picture of quantum particles that has been given so far in this section is one where they are observed to be flashes of energy that are nearly durationless. Particles do not exhibit a continuous existence, and for these reasons, particles do not exhibit the sorts of properties that material items do.

\title{
1.1.9 What about string theory?
}

Many physicists that are called string theorists attempt to argue with sophisticated mathematics that reality is not composed of point-like quantum atoms, but rather of 1-dimensional vibrating strings of energy or 2-dimensional vibrating sheets of energy. The appeal to this theory is enormous, as string theory may give rise to one of the greatest revolutions in science. But in this subsection, I will briefly show why I do not see how I can avoid the conclusion that string theory is in need of one modification: the fundamental items in string theory can only be points, not the aforementioned strings or sheets. My reasoning in sections 3 and 4 of this article show that 1- and 2-dimensional items cannot exist. But in addition to those reasons in Sects. 3 and 4, there is another significant problem for the 1- and 2-dimensional items of string theory that I can point out here, and which I will do in this subsection. Due to the incredible popularity of string theory, and the obvious opposition it has with the mereological nihilist interpretation of quantum physics, it seems worthwhile to point out at this early in this article why string theorists may have a few problems to address that mereological nihilism avoids.

Before the 1900s the particles at the basic level of reality were thought to be either point-particles or Democritean (voluminous) atoms. But with the onset of quantum mechanics there arose a third option: string theory. We are told by string theorists that there is nothing more fundamental than these strings (which to my knowledge indicates that they are apparently basic building blocks), as Matthieu and the physicist Thuan write:

\footnotetext{
${ }^{30}$ Herbert $(1989,160)$.

${ }^{31}$ Ibid.

型 Springer
} 
At the moment we don't know how to unify the twentieth century's two great physical theories-quantum mechanics and relativity. The former describes the... [very] small, and account for the behavior of atoms and light when gravity isn't dominant. The latter describes the... [very] large, and allows us to understand the universe and its structures at a cosmic scale, where the two nuclear forces and the electromagnetic force are not predominant...

Some physicists, who are working on the theory of superstrings,... say that... elementary particles are created by the vibration of tiny "strings" of energy that are Planck length long. Because nothing can be smaller than these strings, the problem of what happens to space on scales smaller than Planck length disappears. Space simply can't have a smaller dimension. This theory seems to have the potential of unifying quantum physics and relativity. But right now it's wrapped in a thick mathematical veil and hasn't been proved experimentally. ${ }^{32}$ (Emphasis added.)

Strings, roughly speaking, are vibrating basic building blocks that (somehow) are theorized to amalgamate and split (apparently when they exist they are basic: the initial string, and the post-split strings, are all basic items). Regardless of what physicists assert, we can apparently find that strings are not fundamental, as I will do next. We are told that the sheets and strings vibrate. And even though string theorists apparently tend to reject the concepts of time and space, ${ }^{33}$ to my knowledge the vibration of the strings and sheets indicates that in some sense they involve persistence: they persist through time. (I only say this because vibration seems to only be a "process" that can occur through time where there is an object that persists while vibrating.) This vibration through time is where the problem I wish to point out comes in. If strings and sheets are considered fundamental, and if their fundamentalness means they are partless, it can be pointed out there is an apparent absurdity involved with the idea that there can be partless items that vibrate through time. So, assuming that I have the account of the string theorists correct, I will proceed.

If, while a string is vibrating, it does so in some sort of wavelike manner, there could be a problem if the waving occurs with more than one wave crest. I don't see how the conclusion can be avoided that such a vibrational string cannot have at least two segments moving in opposite directions, such as when in the act of vibrating, the top half of a string that is vertical with respect to an observer moves to the left, and the bottom half moves to the right. It appears to me that the only way this can occur is if the energy string (and the same would hold for an energy sheet) has parts that compose it, so that the bottom and top, for example, of the string can move in opposite directions. For these reasons, and for reasons pointed out in Sects. 3 and 4, I do not consider string theory in this article, as it appears that the superstrings the string theorists discuss are not partless building blocks, which would be a problem if the strings are supposed to be truly fundamental, as the passage above from Matthieu and Xuan indicated: the parthood of the strings would apparently indicate that there is something more fundamental than the strings

${ }_{32}$ Matthieu and Thuan (2001, 27-28).
${ }^{33}$ See Greene (2004). 


\subsection{Quantum abstract atomism}

Now that I have outlined some of the basic conceptual issues surrounding the experimental data of quantum mechanics, I will use the rest of this introductory section to discuss the philosophy of mereological nihilism.

If mereological nihilism is correct, quantum philosophical atoms do not make up any sort of composite entity, such as a tree, a mind, a human body, a molecule, or a universe. The quantum philosophical atoms never converge, act, ${ }^{34}$ contact, or connect in any way in which they give rise to a mereological whole that is made of the philosophical atoms. ${ }^{35,36,37}$ Mereological nihilism is an attack on all philosophies of mereology and material constitution. "[T]he term 'mereology' [refers to]... any formal theory of part-whole and associated concepts.... ... '[M]ereology'... literally [means] 'science or theory of parts'...",38 It is one of the three areas of philosophy and conceptual physics that attempts to explain material constitution (also referred to as material composition), where topology and mereotopology are the other two-and all of which I show are impossible. ${ }^{39}$

As pointed out in Sect. 1.1, the philosophy of mereological nihilism is also a philosophy of quantum philosophical atomism. In this article I will refer to the philosophical atoms of mereological nihilist reality as quantum abstract atoms. These are the aforementioned partless, momentary point-sized, non-material particles of physics that physicists have discovered: electrons, quarks, gluons, etc. Usually these partless particles, that I am calling quantum abstract atoms, are just referred to as subatomic particles, or point particles, which are terms scientists and philosophers

${ }^{34}$ I use "act" specifically because another nihilist (but which I describe in this article as a quasinihilist), van Inwagen uses the concept of activity in his somewhat nihilistic analysis: it is by some activity of partless subatomic particles that they give rise to a composite item (if, that is, particles can give rise to composites).

${ }^{35}$ Some readers may wonder why reality cannot be atomless, where there are parts "all the way down." This position is against mereological nihilism, since mereological nihilism rejects for any parts whatsoever. According to the philosophy of atomlessness, any material object or topological region can be described as an infinite regress of parts: a spatially extended object can be divided into halves, each half can be further divided into quarters, each quarter into eighths, ad infinitum. The atomless object is never divided down to atoms and involves no atoms (and it is interesting how often philosophers miss this point, for example: Sider $(1993,287)$.) and each part is extended. (See Pyle 1995, 2-6 for a very lucid explanation of these details to do with atomlessness). But if mereological nihilism is correct, I will specifically point out in a section below why atomlessness is an incorrect theory, since atomlessness involves composition and mereology, which I argue in this article does not exist.

${ }^{36}$ In this article I limit my discussion to partless quantum objects, and I do not discuss other possibly partless objects, such as platonia or Armstrongian universals (if Armstrongian universals or platonia are partless, and if Armstrongian universals or platonia even exist [See Grupp articles 2003-2006 for arguments against the existence of platonia, mind-independent $\mathrm{n}$-adic properties, and against any metaphysical objects whatsoever]).

37 The reader can probably tell at this point in the article that by "part" I am referring to "proper part." Proper parts are not identical to the whole, and thus are less than the whole (Simons 1987, 11). An improper proper is one where it is a part identical to the whole ( $\mathrm{x}$ is a part of itself). Mereological nihilism, then, is the position that there are no objects that have proper parts.

38 Simons $(1987,5)$.

39 The reasoning below in Sects. 3 and 4 shows that any other theory of constitution that may be invented in addition to the three just listed are impossible, since the arguments in Sects. 3 and 4 below show that the quantum atoms must exist (i.e., any reality can only be composed of them), and necessarily they cannot interact in any way with one another. 
typically use to refer to all items of the quantum world, whether partless or composite (this is true for all quantum items except the scientists' atom, which is conceptualization of a non-existent composite item allegedly composed of electrons and a nucleus, and is dissimilar to the historically old concept of a philosophical atom). Protons (which have a diameter and are allegedly mereological wholes) and quarks (which have no diameter and are partless) each involve the inverse properties of each other in many significant ways. For that reason, I am not sure why it could be considered a good idea to fit them both under the same label, "particle," or "subatomic particle," given their rather extreme disparateness. Due to the importance of the quantum atoms (as opposed to the quantum composites that are alleged to exist), due to the specific idiosyncratic properties of the quantum atoms, and due to the ways that quantum atoms differ from any compose items (such as the former being real and the latter being non-existent), it appears that a renaming of the partless quantum objects could prove useful for philosophical analysis. After the quantum revolution, no name was adopted to specifically denote the idiosyncratic details of the partless fundamental particles, and to also denote them as immaterial points of momentary energy. "Atom" appears applicable since the partless quantum objects are atomic (in one of the original meanings of the word "atom" (partless object)). (I explain the various reasons why there is reason for quantum mereological nihilists to espouse the word "atom" at many places in this article.) Mereological nihilism is a quantum mechanical philosophy, since the fundamental level of reality, which is empirically shown to be obviously quantum (i.e., nonclassical, involving randomness, non-mechanistic, non-local, non-material). For that reason, what is needed in a renaming is a retreat from the Greek connotations, and antiquated classical and mechanistic connotations of the word "atom," to the empirical atomism of modern quantum physics. The most appropriate word I can think of to denote the unusualness of the non-mechanistic quantum atoms is the word "abstract," since that word could be considered broad enough to denote many aspects of the non-logical (i.e., non-mechanistic), non-solid, simple (partless), nonlocal (non-separable), immaterial, and momentary (instantaneous) quantum flashes of energy. ${ }^{40}$ That is reason for my employing the name quantum abstract atoms, which is meant to denote only such items, and for other reasons I will give throughout this article (and which I have elaborated on elsewhere ${ }^{41}$ ), this name seems most appropriate. This renaming is meant (i) to bring atomism out of being thought of as primarily a mechanistic, medieval or ancient theory where atoms are imagined to behave in the ways that ordinary empirical objects do but only on a much smaller scale, and (ii) to emphasize that mereological nihilist atomism appears to be the best description of the experimental empirical findings in quantum physics.

\subsection{Philosophers and the quantum revolution}

I will show that the experimental data of quantum physics (as distinct from the interpretation(s) of the data of quantum physics) strongly supports mereological nihilism. (In this article, I will only discuss one of the interpretations offered to us by physicists - the positivistic version of the Copenhagen Interpretation-which I do in

\footnotetext{
${ }^{40}$ I adopt the phrases "flashing energy" from a passage by the famous physicist, Herbert $(1989,160)$, and from the famous Buddhist philosopher Stcherbatsky $(1962,19)$.

${ }^{41}$ Grupp (2005a), section 5.1.
} 
6.1 below, due to the fact that it comes to a few of the same conclusions about fundamental reality that mereological nihilism does.) The vast majority of philosophers do not properly acknowledge the implications that quantum physics has to philosophy, and how quantum physics draws into serious question the correctness of ordinary empirical experience. They have not given sufficient consideration to the seriousness of the conflict between the objects of empirical experience and the quantum objects that are supposed to compose those empirical objects that are given in empirical experience. There has been a revolution in science, called the quantum revolution, that the majority of contemporary philosophers in the Western tradition appear to have distanced themselves from. Philosophers have instead all-too-often chosen to align themselves with ancient and pre-scientific metaphysical theories of reality (such as Aristotelian substance theory), and with mereological and metaphysical viewpoints of reality that involve rather obvious and stark problems, antinomies, and paradoxes (such as identity through time and change, ${ }^{42}$ Zeno's Measure Paradox, the paradox of material constitution, just to give merely a few examples). Put in different words, rather than an aligning with the quantum revolution, it appears that a significant percentage of philosophers instead resort to describing reality in terms of non-scientific, extrasensory, metaphysical items and ideas in their description of reality: platonic entities, possible worlds, four-dimensional world-lines, thin or thick particulars, space, souls, God, mind-independent nadic properties, etc. As for philosophers who are metaphysicians, they ask us to believe that there are items such as these, all of which no one has ever perceived, and which they would tell us nobody ever will (but which are needed to make realist metaphysical philosophy coherent), and they recommend to us that we not be overly worried about the data of quantum physics, nor about the immense conflict its empirical findings have with their metaphysical realism. The thesis of mereological nihilism involves a rejection of all metaphysical and non-empirical items, and an alliance with the empirical findings of quantum physics. Quantum physics is perhaps the simplest and most straightforward science (it is "perhaps the most successful theory in the history of science",43). In overlooking or ignoring quantum physics, far too many philosophers often fail to take note of how well-developed the experi-

\footnotetext{
${ }^{42}$ In Grupp (2005a) I discussed how the problem of change is still as much of an unsolved problem as ever for Western philosophers, who nevertheless debate it endlessly with no sign of a solution in sight. (See Loux 2001, especially p. 321.) The problem of change-how can object A remain itself if it changes-has prohibits change from involving endurance or perdurance. The problem, however, is treated in Western philosophy as if it is not really so much of a problem (if the problem was taken seriously, the endurance and perdurance debate would not be options to debate). Consequently, there is no coherent account of change since the problem of change is apparently an unsolvable paradox. Rather, if philosophers want to describe change only do so in the following way: demand that we see that the problem of change is not so much of a problem at all, and imagine that there are enduring or perduring objects. Consider this passage from Zimmerman, where he is writing about David Lewis's complaints about presentism, and notice how the terms "incredible," "obvious," and "obviously" are used:

But, by Lewis's lights, presentism is too incredible to be believed...Presentism "rejects endurance; because it rejects persistence altogether",... And yes, says Lewis, the presentist denies these obvious facts... Lewis takes it that the following thesis of "Persistence through Change" is obviously true: ...There are (at least) two different times; one at which I am bent, another at which I am straight. (Zimmerman 1998, 213)

${ }^{43}$ This is the first line of Faye (2002). Also consider Greene's comment about this: "Eight decades of experiments have shown that the predictions of quantum mechanics are confirmed to spectacular precision." $(2004,90)$

Springer
} 
mental data of quantum physics is, and by doing this, I cannot see how to avoid the conjecture that many philosophers are in some sense trying to find the ether.

Philosophers largely continue to discuss fundamental reality in classical (mechanistic, pre-quantum) terms, often with 17 th century, scientifically outdated classical concepts (I will give examples in later sections) - and even though classical interpretations of the quantum domain cannot provide a single prediction regarding the quantum abstract atoms, whereas non-classical quantum descriptions are astonishingly precise in the predictive power they provide. (This makes it all the more surprising that philosophers are often even disdainful of quantum physics. ${ }^{44}$ ) It may be surprising to many philosophers that ordinary empirical objects are theorized by mereological nihilists to be illusions, but, as I will discuss in detail, mereological nihilism is not any sort of assumptive or careless thesis, since it is the macroscopic ordinary empirical domain (which is only mental conceptualization) that involves absurdity, and also since when humans probe fully into matter, we find that empirical objects are composed of these quantum abstract atoms. When humans fully dissect any of the material objects they conceptualize from moment-to-moment, they only find their concepts to be nonsensical (images of what is not real), where they can only be non-existent concepts (i.e. illusions), that necessarily reduce into unstructured points. Therefore, the conclusions of the mereological nihilist are the inverse of the standard position: philosophers ubiquitously assert that macroscopic reality exists and is coherent (but I will show that it is really antinomic, paradoxical, and non-existent) and quantum reality is paradoxical but somehow composes macroreality (but I will show that quantum reality is non-paradoxical and cannot compose anything).

There may be many reasons why philosophers often do not pay sufficient attention to the quantum data (for example, I have never seen philosophers, other than maybe a few dozen philosophers of physics, discuss the Aspect experiment, quantum entanglement, and so on). Perhaps one reason is that a significant percentage of philosophers do not know enough of the physics involved in the quantum theory, ${ }^{45}$ and they consequently have not seen with their own minds the empirical power of the theories. This could lead philosophers to not sufficiently understand why quantum physicists have come to the empirical descriptions about reality that they have. Or, perhaps many philosophers often will only hear about or pay attention to some of the philosophical theories and interpretations of a few of the high-profile quantum physicists (rather than the raw experiential data produced overall throughout the vast discipline of quantum physics) but where the "philosophical theorization" is shoddy, and thereby philosophers justifiably turn away from

\footnotetext{
${ }^{44}$ The only philosophers I have seen go against this trend, and which are taking part in the quantum revolution, are some philosophers of physics, such as those who focus on philosophical issues in quantum mechanics. A good example is Lockwood: "The scientific picture that emerged in the 17th century has, I believe, been effectively shattered by Einstein, Bohr, Heisenberg, Shrödinger and Dirac. And philosophy needs, however belatedly, to come to terms with this scientific revolution." (Lockwood 1989, 8)

${ }^{45}$ It is worth noting that I also do not have the training that a professional physicist has. In this article, I will heavily cite physicists and philosophers of physics due to the fact that I am not a trained physicist, and due to the fact that many of the philosophers who read this article will not be either. It should also be noted that some of the physics books I am quoting from are not overly technical books but rather are books with more general information about quantum mechanics. But all the passages from physics books that I cite are only from some of the most prestigious physics professors in the world.
} 
quantum theory due to the amateurishness of the few high-profile philosophical theories (e.g., wave-particle duality). ${ }^{46}$ Or perhaps many philosophers will hear about the so-called paradoxes of quantum mechanics, such as wave-particle duality, and since allowing reality to contain objects that involve contradiction goes strictly against their philosophical training, philosophers may therefore turn away from quantum physics, and instead of accepting paradox and contradiction in reality the philosphers may espouse the belief that physicists merely have run up against apparent paradox because they do not yet have a good enough understanding of the quantum world. Another reason that philosophers ignore quantum physics might be because a few famous quantum physicists have (quite amateurishly) been mudslinging vocalizations of their utter contempt toward philosophers. Some famous quantum physicists have literally been observed to frequently berate philosophers as being thoughtless buffoons, and, for example, as being inappropriate in their merely attempting to question quantum physicists on things like the so-called paradoxes of quantum mechanics. Perhaps the best example of this was Nobel Prize quantum physicist Richard Feynman, who is famous for many of his frequent extremely derogatory, but unsubstantial (i.e. non-inferentially based), remarks against philosophers (even incorporating them into his undergraduate lectures), for their merely attempting to carry out investigations of language and thought, and for their having justifiable questions about the philosophy of paradox. Even Einstein, who was known to be very much indebted to philosophers, took jabs at them. ${ }^{47}$

Bringing philosophy and quantum physics into collaboration can only occur if quantum physics is not considered to be a set of disparate interpretations that a few high-profile quantum physicists have (unfortunately) given the world, but is considered to be empirical, based primarily on confirmation via experiment. For purely empirical purposes, the interpretations of the data are largely irrelevant. It is the experimental findings in quantum physics that I will focus on in this article when I discuss issues to do with quantum physics. And I will find that quantum experimentation is one of the reasons to conclude that mereological nihilism is the correct theory.

\subsection{Empirical versus quantum}

The only real objects that the experimental findings of quantum reality reveal to exist are particle-like point-objects. An item such as the scientists' atom, which is alleged to be a composite with a nucleus and one or more electrons, will be shown to not be a real entity by the evidence of this article. The experimental findings show that the ordinary empirical experience that humans ordinarily have involves serious error. Nevertheless, in the literature, philosophers in all areas of philosophy (including philosophy of physics) typically discuss ordinary macroscopic empirical experience as if there is no reason whatsoever to be suspicious about it. In writing

\footnotetext{
${ }^{46}$ A great example is the Shrödinger's cat problem (which apparently was done away with by the discovery of decoherence, according to Omnés (1999a, ix)). Many of the questionable interpretations are beginning to be rejected more by physicists (Stenger 2000,14-15). It is important to note that many physicists take issue with some of the ways that the interpretations of quantum physics have proliferated. Omnés, for example, refers to some of them as "wild speculations" and as "a dangerous trend, as much for the public at large as for philosophers, who may have difficulty finding their way, especially since some reputable physicists are among the [speculators]," (Omnés 1999b, 147).

47 Einstein $(1950,2)$.

型 Springer
} 
about mereological nihilism, one of the main objectives I have in this article is to show what a mistake it is to believe that ordinary macroscopic empirical reality is real and exists in the way that humans experience it.

Quantum physics is empirical, but is of a different, more refined, scientific order of empirical experience that macroscopic experience is. Quantum empirical data is not of the same order of empirical experience as the everyday, unrefined, macroscopic empirical level, which is so prone to error and deception. Here are a few examples of the common error and deception that ordinary empirical experience involves: continuous pitches of sound, smooth glass surfaces, mirages, twinkling stars, movies (successions of still frames interpreted as involving motion), leaves appearing to have continuous green color that is devoid of interstices, long rectangular airplane propellers that change into a nearly invisible disk when they are turned on, straight light rays that look curved when shined through falling elevators, ${ }^{48}$ solid rocks, train tracks meeting at the horizon, colorless-empty interstellar night skies that however appear black, military planes that look like UFOs, blue skies - and that is just the beginning. In each of these examples, the macro-oriented empirical mind perceives items that do not exist. The ordinary macroscopic empirical level of reality that humans ordinarily perceived is riddled with dubiety. Mereological nihilism is not about the rejection of the empirical, it is about the rejection of all empirical items except quantum atoms, as I will elaborate on in this subsection.

Mereological nihilism involves an assault on ordinary empirical experience, and the objects believed to exist according to that experience. ${ }^{49}$ I will discuss in detail how quantum reality and ordinary empirical realty are the inverse of one another. Ordinary empirical reality is dominated by the so-called classical concepts, which are concepts that describe material items as existing in time, having a definite position, entering into causal relations with other objects, having a surface extension, and so on. A particularly lucid passage from Faye helps to understand the ideas behind, and the history of, classical concepts:

Not unlike Kant, Bohr thought that we could have objective knowledge only in case we can distinguish between the experiential subject and the experienced object. It is a precondition for the knowledge of a phenomenon as being something distinct from the sensorial subject, that we can refer to it as an object without involving the subject's experience of the object. In order to separate the object from the subject itself, the experiential subject must be able to distinguish between the form and the content of his or her experiences. This is possible only if the subject uses causal and spatial-temporal concepts for describing the sensorial content, placing phenomena in causal connection in space and time, since it is the causal space-time description of our perceptions that constitutes the criterion of reality for them. Bohr therefore believed that

\footnotetext{
48 This is a famous example from Einstein's falling elevators examples. See Sklar (1977), 69.

49 I use the word "assault" to make the point of how important it is to quantum mereological nihilism that faith in ordinary macroscopic sense information be attacked and challenged. In this article I will point out many problems to do with physics and philosophy, but we will see that perhaps nearly all of which stem from one simple issue: oddly, philosophers and physicists have tended to not consider the possibility that the way they ordinarily experience reality is radically incorrect, and that there is a difference from reality as it is ordinarily empirically perceived to the way reality really is. In my dealings with philosophers and physicists, I typically perceive in them an extreme resistance to these ideas-ubiquitously they appear to not open up to the obvious evidence that ordinary empirical experience involves significant flaws and needs to be seriously questioned.
} 
what gives us the possibility of talking about an object and an objectively existing reality is the application of those necessary concepts, and that the physical equivalents of "space," "time," "causation," and "continuity" were the concepts "position," "time," "momentum," and "energy," which he referred to as the classical concepts. He also believed that the above basic concepts exist already as preconditions of unambiguous and meaningful communication, built in as rules of our ordinary language. So, in Bohr's opinion the conditions for an objective description of nature given by the concepts of classical physics were merely a refinement of the preconditions of human knowledge. ${ }^{50}$

The concepts for quantum reality, as I will discuss, involve a rejection of all of the classical concepts. In other words, what holds for the classical level is the inverse of what holds for the quantum level: the quantum level is non-classical. On the opening page of the Preface of his 1987 book on quantum logic, Gibbins writes:

Quantum mechanics is most easily interpreted antirealistically, that is, as a theory which, though it works, does not describe the way the world is. Therefore, philosophers go out of their way to interpret it realistically. Realism in the philosophy of quantum mechanics means the idea that quantum systems are really like classical particles. Everything points against it. ${ }^{51}$

Also consider an interesting passage from the famous physicists, Davies and Brown:

The discoveries of the electron and of radioactivity, the success of Planck's quantum hypothesis and the inception of Einstein's theory of relativity swept away the entire basis of Newtonian-Maxwellian physics. Newton's laws of motion and his commonsense assumptions about space and time were abandoned. Even Democritus' atomic hypothesis had to be replaced by a more subtle and complex view of the microworld in which atoms could no longer be regarded as indestructible particles with a well-defined position and motion. It became apparent that the foundations of classical physics had collapsed.

By about 1930 their place had been taken by new theoretical schemes: quantum mechanics, the general theory of relativity and a more elaborate model of the atom. $^{52}$

Before the quantum revolution, the classical concepts were also believed to describe the partless fundamental particles. That ended with the discovery that the basic level of reality is governed by quantum (non-classical) concepts. Therein came the following unsolved inconsistency: how do the quantum atoms (allegedly) compose empirical reality when nothing about the quantum abstract atoms appears to allow for them to do so. A principle tenet of quantum mereological nihilism is the idea that the quantum abstract atoms cannot compose empirical reality (and they cannot compose anything at all). This inconsistency only arises when one believes that the quantum abstract atoms are supposed to compose the empirical level. Asserting that they do is analogous to the assertion that a human society can be made out of cats,

\footnotetext{
${ }^{50}$ Faye (2002), end of Sect. 2.

${ }^{51}$ Gibbins (1987, ix).

52 Davies and Brown $(1988,4)$.

笔 Springer
} 
that ice can be made of fire, or an ocean can be made of dirt. In each of these cases, the constituents cannot lead to the constituted; the constituents hold incompatible properties with what's needed to construct the constituted.

Quantum mereological nihilism is obviously a philosophy that is at odds with the ordinary material world that humans (believe they) experience in their daily lives. ${ }^{53}$ For reasons I will discuss, this is a strength of mereological nihilism. I mean the word "material" in the previous sentence and throughout this article to denote the objects in reality that humans ordinarily believe exist in the world outside of them, and which is empirically perceivable "stuff" that allegedly exists in space (has a spatial location), has a spatial extension (i.e., has a surface and/or a color), and endures (or perdures) through time without flashing in and out of existence (without quickly coming-to-be and passing-away). These characteristics of matter written in the previous sentence all lead to the famous antinomies and problems of paradox and vagueness that are all-to-well-known in philosophy. They are not characteristics of the particles of quantum physics, since the particles have no location, ${ }^{54}$ have no enduring existence (they have no significant duration), no structure, they do not touch, they exhibit little or no movement, and they do not have spatial extension (they are not observed to have surface, and thus no color, and quantum abstract atoms cannot be directly empirically observed at all, as I discuss below) But the current landscape of philosophy is replete with realists (naïve realists and direct realists, metaphysical realists, semi-realists, etc.) who invent theories of reality-in direct opposition to the mereological nihilist-based primarily on faith in the existence of the material world that humans believe they experience. I will use the terms "realism" or "realists" in this article to refer to any of the theories of realism, and to refer to any philosophers (or scientists) who unquestioningly accept the information of

\footnotetext{
53 Just to avoid any possible confusion that could arise, I want to point out that I am going to use the word "experience" throughout this to be synonymous with "consciousness." Consider how Galen Strawson uses the word "experience":

I want to establish a certain broad use of the word 'experience'. In talking about mental goings-on, I will rarely use the words 'conscious' and 'consciousness'. Instead I will talk of experience: of experience in general, of experiencers, particular experiences, experiential states, experiential episodes, experiential phenomena-human, Martian, dolphin, canine, and so on. I will use the term 'experience' to cover everything that philosophers usually have in mind when they talk of consciousness or conscious experience, taking it that "the stream of consciousness" could equally well be called "the stream of experience" and that the expression 'conscious experience' is, strictly speaking, pleonastic. (Strawson 1994, 2-3.)

${ }^{54}$ The casual reader may believe that I am making this assertion due to quantum uncertainty, according to which location is not clearly defined for particles. But this is not what I mean by asserting that a quantum abstract atom is locationless. What I do have in mind, rather, is that atoms are locationless because space does not exist, and it doesn't because it is an item that (allegedly) has parts. In Grupp (2005b) I showed that space does not exist. I come to the same conclusion in this article but with very different arguments. The philosophy of spacelessness I have in mind (mereological nihilist spacelessness) is loosely similar to the widely discussed relationalist position since both mereological nihilism and relationalism involve the non-existence of space; but, as I will explain, mereological nihilist spacelessness has stark differences with relationalism (such as that relations do not exist in mereological nihlism). Space is an invisible (metaphysical, extrasensory) item that, I will show, only leads to unneeded conceptual difficulties, such as quantum uncertainty. (I will show in a later section that the concept of quantum uncertainty is misguided since without space and locatedness there is no location, $x$, in the uncertainty equation, $\Delta x \Delta p=$ (Planck's Constant)/2.) In this equation, $x$ is merely a concept.
} 
their ordinary empirical experience to be approximately correct or completely correct. $^{55}$

The true scientist will often choose to reject some of the empirical experiences she has as being false. For example, the acoustical scientist will have daily ordinary empirical experience of continuous pitches of sound that appear to her as smooth and unbroken, but she will likely nevertheless reject them, and consider them illusory, since her training as a scientist shows her that pitches are discontinuous, regardless of what her ordinary empirical experience reports to her. Similar reasoning could be given for light, which is associated with another one of the most common ordinary empirical experiences (sight experiences). Sight and sound dominate ordinary human empirical experience with what illusorily appears to be chunks of smooth, unbroken, continuous streams of information. But what I am calling a realist is any person who is prone to merely accept obviously questionable empirical experiences (e.g., "continuous" light, sound) as being reliable (correct) accounts of reality. Given the current wild popularity of the theories of realism, it can be predicted that in the current zeitgeist in philosophy, mereological nihilism will likely be rejected without argumentation due to the realists' preference for, and the desire for, realism-but where this is only an issue of preference rather than inference.

Mereological nihilism involves the position that not only are all such realist philosophies to be rejected, but also that all scientific theories and scientific items (items scientists perceive in order to do their scientific work) are to be abandoned if they are not scientific accounts of real items (of quantum abstract atoms) ${ }^{56,57}$ Only science and philosophy that involves quantum abstract atoms is admitted. The quantum abstract atoms are, however, not directly empirical, but rather can be considered to be indirectly empirical. What I mean by indirectly empirical is given in an example: If I am in the glade and I see the footprints of a panther in the mud, though there are no panthers around, I surmise that one was recently. I do not see a panther; it is an unobservable. But I nevertheless have an experience that lets me know a panther was present. In other words, I have indirect empirical evidence for the existence of a panther. This is how physicists know about quantum abstract

\footnotetext{
55 Theories of realism in philosophy tend to be highly metaphysical (as I will discuss), and are very often aligned with what is called metaphysical realism. In the Cambridge Dictionary of Philosophy (Cambridge University Press, 1995) pp. 562-63, Butchvarov describes what is meant by "metaphysical realism":
}

\begin{abstract}
Metaphysical realism, in the widest sense, [is] the view that (a) there are real objects (usually the view is concerned with spatiotemporal objects), (b) they exist independently of our experience or our knowledge of them, and (c) they have properties that enter into relations independently of the concepts which we understand them or of the language with which we describe them. Anti-realism is any view that rejects one or more of these theses, though if (a) is rejected the rejection of (b) and (c) follows trivially...
\end{abstract}

Also, for an excellent description of what is meant by "realism," see Dreyfus $(1997,54)$.

56 This sentence shows how much affinity mereological nihilism with ancient Buddhism, since this sentence in some ways reveals core issues involved in traditional Buddhist ethics.

57 This sentence only is meant to indicate that there should be an abandonment of all non-quantum science when one is not tied to, not subservient to, the ordinary empirical material level of experience. If one is trying to understand ordinary empirical reality, the history of science shows that it is the best way to do that is via scientific means. But if one aptly understands the dubiety of the material-empirical realm, then one has little choice but to endorse a rejection of it. Quantum reality is the inverse of this material realm, and dubiety of matter should lead to trust only in the quantum. 
atoms: they do not see them directly, but they see their "footprints" on the computer monitor. ${ }^{58}$ Since no metaphysical item is indirectly empirical in this way, then quantum abstract atoms cannot be classified as metaphysical objects even though they are invisible. Throughout this article I will refer to the quantum abstract atoms as being I-empirical, for reasons just given.

\subsection{The futility of anti-reductionism}

Anti-reductionists put forth the presumption that somehow there are ways to theorize about these two levels-the quantum and the macroscopic, which have properties that contradict one another-where it can be shown that quantum items must compose the empirical macroscopic bodies. This is the anti-reductionist position, which is describable in the way I will explain next. Empirical object E might be composed of more than one quantum system: E can be made up of quantum state $\mathrm{Q}_{1}$, but if those quantum particles were replaced by all new particles and/or a new quantum state $\mathrm{Q}_{2}$, E would appear to the ordinary empirical experiencer as the same object $\mathrm{E}$ through both states $\mathrm{Q}_{1}$ and $\mathrm{Q}_{2}$, and therefore $\mathrm{E}$ is not reducible to the quantum states. Consider the following diagram:
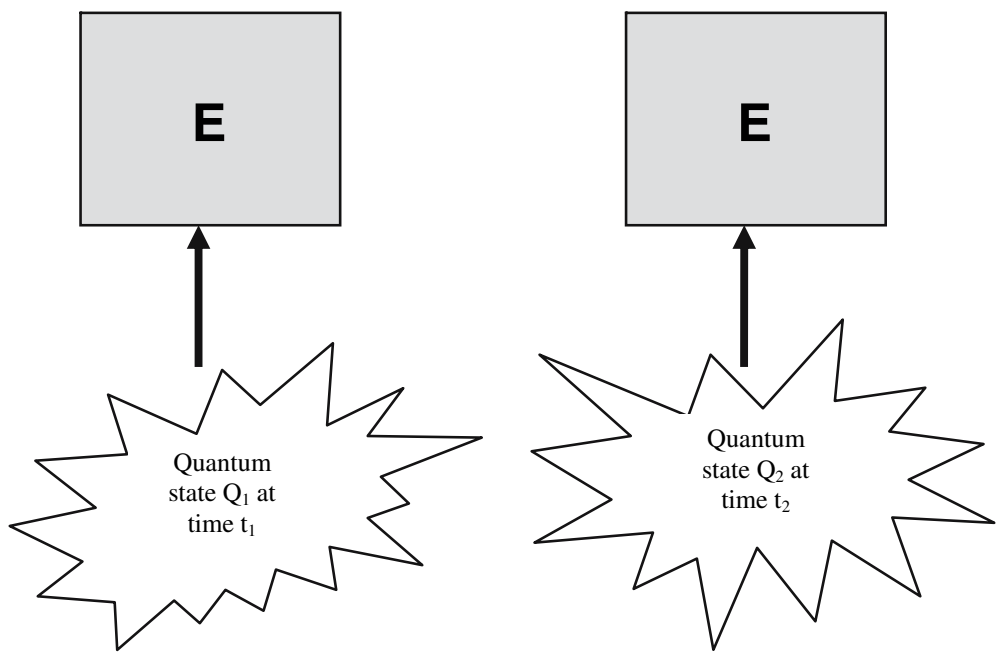

$$
\mathbf{Q}_{1} \neq \mathbf{Q}_{2} \text {, but } \mathbf{E}=\mathbf{E} \text {. }
$$

This is much like the problem of the ship of Theseus: parts of E are coming and going, but E (allegedly) persists, and remains identical through time. The argumentation I give in Sects. 3 and 4 shows that $\mathrm{E}$, in the example given above, cannot exist.

The belief that there is "independence" that $\mathrm{E}$ (allegedly) has from $\mathrm{Q}_{1}, \mathrm{Q}_{2}$, or any quantum abstract atoms that (allegedly) compose it, is easy to understand if we consider, for example, a lion, $\mathrm{L}$, that is believed to be composed of quantum abstract atoms. L might often appear to the ordinary empirical observer as being the very

58 How it is that a scientist can have knowledge of a particle (real item) via a computer or machine (composite and macroscopic item, and thus not a real item) is discussed in an footnote below in Sect. 2.4 . 
same object from moment to moment. But quantum physicists show us that the particles that make up L are going through tremendous activity (flashing in and out of existence, or if they are moving, they are moving near the speed of light, etc.) all while the ordinary empirical observer observes L empirically to not change much, if at all-and thus L appears to persist through time. Anti-reductionists might take their ordinary empirical experience of the alleged persistence of $\mathrm{L}$ to be verification that L persists, regardless of what quantum abstract atoms are "doing" from moment to moment. Anti-reductionists might take this to show that the quantum level is independent of the quantum systems that (allegedly) compose it. Also, if E is believed to exist and is constituted by more than one quantum state, is nevertheless unclear how this alleged "multiple-realizability" implies that E is not reducible to its particles. I am apparently not alone on wondering how this is the case. Jones writes:

One of the difficulties with discussions of MR [the multiple realizability argument] is that few writers have ever tried to spell out precisely what it amounts to. "Indeed, as far as I know," writes Lawrence Shapiro, "no philosopher has ever tried to complete the sentence, ' $\mathrm{N}$ and $\mathrm{M}$ are distinct realizations of T when and only when-' (2000, 636). I find a similar lack of precision in philosophers' discussions of how the existence of MR is supposed to show that physical reduction is impossible. ${ }^{59}$

To sum up the problem that anti-reductionism involves, consider that if $\mathrm{E}$ is not identical to the ultimate parts (quantum parts), and in some sense transcends any parts (it may be believed to have), but is a whole object not reducible to the ultimate parts, then E, if not parts, is in some sense partless. This indicates that $\mathrm{E}$ has parts but can only be partless, which is a contradiction. These issues will be elaborated on extensively in Sect. 4.

Lastly, there are more serious problems for the anti-reductionist. How can a metaphysical relation of part-whole connect parts and the whole? The single relation must connect a partless object (whole) to parts, and thus it must do some rather interesting things, such as connect up to an extended single item (the whole), and to a multiplicity of items (the parts). How does a metaphysical relation do this without itself having parts? How can a metaphysical relation connect to distinct items without itself having distinctions "within" it? If the metaphysical part-whole relation has parts, then serious questions arise as to how the relation can itself be a mereological whole. The metaphysical part-whole relation would have to have a part that, for example, connects to part \#1 of the whole, and since part \#1 is not where part \#2 is, then the relation would have to have a separate non-coinciding part of it that connects to part \#2 of the whole. In other words, the two parts are not coinciding, and thus if it the relation links up to each of them in order to connect them to the whole then the relation has noncoinciding aspects of it. (Again, I will show why there are no wholes with perfectly coinciding parts in Sect. 4.) But if that is the case, then this relation that itself has parts also is in need of a mereological relation, call it $R_{2}$, that connects part \#1 and part \#2 of $R_{1}$. But these non-coinciding parts, \#1 and \#2, require that $R_{2}$ have distinct non-coinciding regions where it links $\# 1$ and $\# 2$, and thus $R_{2}$ is a composite that is in need of a mereological relation, $\mathrm{R}_{3}$. This sets up an infinite regress, and there is never a step in the regress that the task of relating parts is

\footnotetext{
59 Jones $(2004,623)$.

Springer
} 
completed since there will always be non-coinciding relation-parts to be interconnected.

The problems all just pointed out could be avoided by maintaining that the metaphysical part-whole relation is partless, but I just showed above that to connect distinct relata, the relation would have to itself involve some distinctions. There seem to be too many problems with the part-to-whole relation for the anti-reductionist to avoid. And matters are only compounded for the anti-reductionist when the additional reasoning I give in Sect. 4, where I show that all metaphysical relations whatsoever do not exist, is considered.

From what has been written here, it seems that anti-reductionism fails to offer any sort of means by which we can assert that non-reducible wholes can be composed of quantum abstract atoms. This reasoning in this subsection about the problems with the mereological relation between non-coinciding parts of any alleged composite item is in fact an argument against the existence of mereological relations, and thus an argument against composition (since composition can only be given in germs of non-coinciding items-see Sect. 4) distinct from, and in addition to, the numerous arguments I give in Sects. 3 and 4.

\subsection{The basics of quantum mereological nihilism}

The heart of my arguments for mereological nihilism consist in showing through several novel arguments that no quantum abstract atoms can touch, contact, connect, or relate to one another (this is given in Sects. 3 and 4). If they cannot, I will show that there is no way that particles can accumulate to give rise to composites, and material constitution is impossible. If there are no parts and wholes, then there are only quantum atoms that cannot touch, contact, relate, or connect, and cannot compose an object made of parts. ${ }^{60}$ Mereological nihilism leads to the position that what actually exists (quantum abstract atoms), what reality is, is both immaterial and Iempirical. ${ }^{61}$ To maintain that is to reject the existence of the surfaces and colors that humans typically believe exit externally to them. Humans experience empirical reality only via surfaces and colors. According to mereological nihilism the surfaces and colors (believed to exist) in external reality are mere concepts in the mind. ${ }^{62}$ Consider the following diagram:

\footnotetext{
${ }^{60}$ Notice that the claims just made in this paragraph begin with the presumption that there are particles in the first place, rather than there being an infinitely divisible reality that ultimately contains no particles. In Sect. 4, however, I will show why reality cannot be infinitely divisible, and thus must involve quantum abstract atoms.

${ }^{61}$ The only tradition I know of that maintains that the quantum atoms can be directly empirical (unmediated objects of consciousness), are some Buddhist traditions. The word for this empirical experience is "nirvana."

${ }^{62}$ Stroll, on page 15 of Stroll 1988, discusses how "some philosophers have held that it is a necessary condition of something's being an object (or what they sometimes call a "physical object" or "material object," of which a marble would be a prime example) that is have a surface." While this is certainly not one of the primary ways that philosophers and physicists have come to define material objects, it is worth noting since it illustrates how what philosophers and physicists often describe as being material has the opposite properties of the quantum abstract atoms. For example, if surface possession was a defining quality of an object for the object to count as a material object, then an electron, for example, would be an immaterial object. This sort of reasoning will be very significant in the discussion in Sects. 2.4, and 5.4 below regarding what I call the philosophy of immaterialism, according to which, reality is composed only of immaterial items, no material entities exist. I will show how quantum mereological nihilism leads to this position.
} 


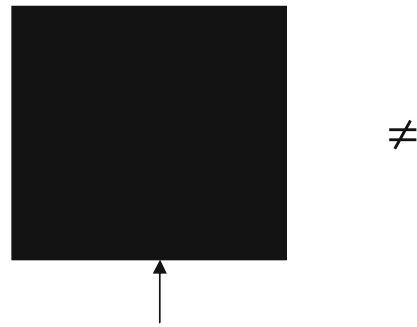

This is how things are observed via ordinary empirical experience: there appears whole (unbroken) items that appear to be individual single items, apprehended by the mind as a surface with a color, with solidity and smoothness, and a definite location.

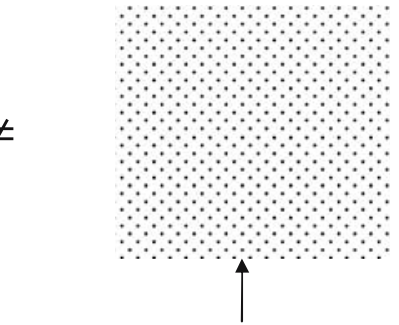

This is more in accord with reality at the quantum nihilist domain: a seeming infinitude of things, each without parts, and each with no surface, color, solidity and there is no smoothness, and no location.

The left side shows how humans ordinarily experience reality, and the right is more in accord with the way that quantum physicists have found reality to be. On the left is a picture of a solid object with a locally smooth surface (an object with no visible interstices); and the right picture does not involve solidity or a surface. (Some readers may imagine that the particles in the picture on the right look like a gasstate. But note that the picture on the right is meant to be a picture of the most fundamental items - electrons, gluons, etc.- known to exist, and thus the picture on the right is not at all identical to the chemical phenomenon that is ordinarily referred to with the term "gas-state," such as when scientists are discussing gasses like air or helium.)

When the typical human looks at a cup on a table in their ordinary empirical state, the cup seems unmoving, solid, and it appears to be persisting through time, but if I peer into the cup to merely see what it is composed of, I would find that the constituents of the cup are not solid, not unmoving, and they do not persist, such as when I am indirectly experiencing, for example, the "electron [which] is a speck of negative electricity that zips around..."63 If an ordinary empirically oriented conceptualizer (which is a word I will use to describe the generator of empiricalmaterial surface and color images in consciousness) has experiences of a cup breaking apart repeatedly (as when peering into the cup-the cup surfaces-to see what they are compose of), where her conceptualization reveals smaller and smaller surface and color bits in her surface and color experiences, she will eventually arrive at the obliteration of surface and color experience-she will arrive at I-empirical quantum abstract atom experience. When having the succession of surface and color experiences with respect to the cup, she will have glass shard surface and color experiences, which will be replaced by molecule surface and color experiences, which be replaced by I-empirical quantum experiences (if she has some means of Iempirically apprehending the invisible items). This series of experiences will occur for any empirical conceptualizer that breaks apart surfaces in their ordinary empirical conceptual experiencing: the surfaces and colors will be obliterated-just as when the physicist happens to peer "beyond" the surfaces and colors. The right side of the diagram is the converse of the left: which is the reason for the $\neq$ between them in the diagram. The quantum stuff that makes up reality is not stable (it only exists in flashes, for exceedingly tiny or instantaneous durations, meaning that it is

\footnotetext{
63 Ford (2004, 2). 
durationless or it does not endure for more than a tiny duration), and it does not have structure or spatial magnitude (it is not observed to have surface, and thus it does not have color).

\subsection{The only coherent theory is banished in western philosophy}

Even though mereological nihilism is strictly aligned with the data of quantum physics, and even though some of the most famous philosophers may have been mereological nihilists or have held positions very similar to mereological nihilism (Democritus, Dharmakīrti, Hobbes, Quine, Sellars, perhaps Kant, etc.), surprisingly, mereological nihilism is currently a minority position, and even more surprisingly, it is, for some reason, often labeled "extreme," or "radical" (textual evidence of this will be given in sections below). There are many reasons why it is very startling that mereological nihilism is not considered the premier theory in contemporary philosophy. This is surprising, as mentioned, due to mereological nihilism's agreement with quantum physics (which is thoroughly explained later), but also due to the enormous fallibility and unreliability of the ordinary empirical-material domain, and due to the antinomies and paradoxes that have always plagued non-nihilistic philosophy (discussed in Sect. 2.2). One would imagine that, in light of much of the data from experimental quantum physics, mereological nihilism would be instead gaining popularity, rather than dropping into the status of being one of the least popular philosophical theories in Western philosophy. (This is not the case in some non-Western philosophy. In writing about the pre-classical Indian Buddhists, Stcherbatsky writes: "For the Buddhists the parts alone are real, the whole is a fiction." $\left.{ }^{64}\right)$. Only a few contemporary philosophers, such as Trenton Merricks, ${ }^{65}$ Jeffrey Grupp, ${ }^{66}$ Cian Dorr and Gideon Rosen, ${ }^{67}$ and Peter van Inwagen ${ }^{68}$ have argued for nihilistic or at least somewhat nihilistic positions in their philosophy. I will show through textual evidence that the vast majority of Western philosophers are unjustifiably and unreasonably disdainful of mereological nihilism, even though, as I will discuss, there is not one noteworthy argument against it that has been given. ${ }^{69}$

\footnotetext{
64 Stcherbatsky $(1962,86)$.

65 Merricks (2001).

66 2005a, b, c, d, 2006a, b.

67 Rosen and Dorr (2002).

68 Van Inwagen (1990).
}

${ }^{69}$ It is not even a secret that there are no arguments against mereological nihilism, which make it very suspicious that there is such pervasive rejection of the theory among Western philosophers. (If there is no evidence against mereological nihilism, then what is the reason for the rejection of it? Non-evidence?) For example, Donald Smith, in a forthcoming paper in Pacific Philosophical Quarterly titled "The Vagueness Argument for Mereological Universalism," writes at the very end of his introduction section that "Throughout, I assume the falsity of mereological nihilism, the thesis that necessarily, there are no composite objects." (Emphasis added.) Notice that he writes that he "assumes" the falsity of mereological nihilism, which is an admission by a non-nihilist that there is no clear reason at all as to why mereological nihilism is incorrect. Also, in his article, Smith merely rejects mereological nihilism for no clear reason. This is the standard way philosophers handle mereological nihilism, as I will show. The widespread rejection of mereological nihilism-which, I will show, is even often filled with statements involving nothing more than non-evidential content involving mere derision and ad hominem - rather than inference and counterevidence-could perhaps lead one to imagine that there is a non-philosophical, non-evidence-based motivation for this widespread rejection. 
Some anti-nihilists have alleged that mereological nihilism is false since it is not in agreement with the exceedingly misleading reality humans apprehend in their ordinary empirical experience, but I will show that this is not a noteworthy attack against mereological nihilism. I will show why there are very good reasons for outright rejection of all empirical experience, except the I-empirical experience of quantum objects through scientific means. Most attacks against mereological nihilism involve no more than non-arguments and/or ad hominem claims that can be easily shown to be informal fallacies (begging the question, red herring, etc.) as I will explicitly show by example.

Some of the reasons for this outright rejection of the ordinary empirical experience of materiality that I just mentioned are actually well-known by philosophers (and some physicists), but are, however, typically ignored and/or derided, for some reason, by philosophers (and even by many physicists). For example, if one tries to describe the most ordinary features of the objects of empirical reality, such as the motion or change of any empirical object, or the identity of an object through change or through motion and time, one finds that they cannot do so without insurmountable paradoxes, absurdities, and antinomies coming into play. These absurdities, paradoxes and antinomies have been widely discussed (but ironically also pervasively downplayed), and have been pointed out by some of the most famous philosophers (Kant, Zeno, Nagarjuna, etc.). These well-known paradoxes, in addition to the impossibility of material constitution that I will show quantum atomism involves, reveal that there is something very erroneous about our ordinary understanding of empirical reality. I will show that the philosophical and scientific evidence for mereological nihilism that I present in Sect. 4 shows that empirical reality is impossible, and thus should be rejected. Mereological nihilism solves the widely known antinomies and paradoxes of philosophy, and, interestingly, also removes the widely discussed quantum paradoxes in quantum physics. It is as if mereological nihilism is the only non-antinomous, nonparadoxical theory in philosophy and science, but it is the least widely accepted theory. This indicates an alarming collective error apparently being committed by the academic community, on the order of past collective errors such as believing in ether or disdaining the EPR thought experiment. These are reasons why it is surprising that the mereological nihilist solution to the problems of physics and philosophy has been ignored by contemporary scientists and philosophers-ignored by the same scientists who in fact have discovered strong evidence for mereological nihilism. Physicists have discovered a mereological nihilist reality in quantum physics through their I-empirical data, but rather than acknowledging that, many have instead tended to add the aforementioned impalpable and supernatural metaphysical concepts into their research-splicing I-empirical discovery with metaphysical fabrication-and only by that means, as we will see, have they arrived as paradox.

In addition to its scientific alignment, and its antinomy-less status, it is perhaps most surprising that mereological nihilism is a banished theory in Western philosophy when we consider that it is no secret that there is no consistent theory of mereology and material composition that is currently available. The mereological nihilist of course maintains that we can only expect this, and expect that no consistent theory of mereology and material composition will be available since those are the wrong theories, according to her. But this is not recognized, and instead, the current state of philosophy is one where mereological nihilism is nearly banished from Western philosophy, and material constitution (which is littered with the antinomies and paradoxes, and which relies on metaphysics and the rejection of basic science, 
and which is "unacceptable", in Markosian's words ${ }^{70}$ ) is accepted. (I will give textual evidence showing that this unacceptableness of the metaphysical mereological and compositional theories is commonly accepted and discussed in the literature among philosophers.) I call mereology and compositional theories metaphysical theories, as if they are in all cases, because I do not know of any compositional and mereological theories that do not rely on usage of relations, emergentism, or in general any of the other metaphysical, extrasensory, non-scientific tools of the realists and metaphysical realists.

What this appears to amount to is this: the academic community of American philosophers involves a majority of philosophers who endorse problem-filled, undeveloped, non-empirical, antiquity-based metaphysical "theories" of material constitution that have virtually no explanatory value (in terms of empirical experience and thus in terms of explaining anything about one's experiences of the empirical universe), and instead they reject and/or ignore the strictly nonmetaphysical, utterly progressive and modern, empirical (I-empirical) scientific results that are perhaps of the most developed and remarkable experimental outcomes in all of science. If this is what so many philosophers are doing, how is this any different from, for example, turning our backs on relativity theory in order to go back and try to find the ether? Or how is that any different than if physicists everywhere chose to turn their backs on the theory of the Big Bang singularity in order to study the intelligent design theory? Or if biologists were to reject evolution in order to accept abiogenesis or the Garden of Eden story? Or if astrophysicists rejected the expanding universe theory for the steady-state universe theory?

It would seem that philosophers would be flocking to mereological nihilism, but instead it is a fringe thesis, and philosophers who study composition nevertheless typically assume or demand that there is composition and/or mereology-which, by comparison, appears to be an anti-scientific, and perhaps to some degree tends toward being an anti-philosophical quest for ether, intelligent design, abiogenesis, the Garden of Eden, and a steady-state universe. Rather than it being the case that the well-known problems with composition and mereology provide evidence for the non-existence of mereology and composition, philosophers instead commonly assume mereology and composition to exist and the problems with them to merely be evidence that mereology and composition are mere puzzles that will be figured out in the future. Consider a passage from Michael Rae, at the very beginning of his anthology on material constitution, and where he discusses composition as a mere puzzle that need not be too troublesome, rather than as an impossibility (as I will show it is in Sects. 3 and 4):

Imagine calling on a friend to collect a debt and receiving, instead..., the following... argument:

....a human being is just a collection of particles... [and] if you add particles to or subtract particles form a collection of particles, you get a new collection. ... I am [now] a different collection of particles form the one that contracted the debt... Thus,... I am a different human being form the one who contracted the debt. Therefore, I do not owe you any money.

Obviously this line of reasoning is flawed... But how it is flawed?... The answer is not immediately clear.

$\overline{70}$ Markosian (1998b, 214-215). 
What the debtor has raised is a puzzle about material constitution... This phenomenon is puzzling because it is not at all clear what the relationship is between the relevant $a$ and $b$ [Rae is using the letters $a$ and $b$ to symbolize the words "whole" and "parts"]...

Puzzles like this are among the oldest, most widely discussed puzzles in the history of philosophy. They have appeared in the writings of Epicharmus, Aristotle, Chrysippus, Abelard, Locke, Leibniz, Hume, and Hobbes, to name just a few. ${ }^{71}$ (My emphasis)

Notice how Rae maintains that "obviously this line of reasoning is flawed," but then two sentences after that he maintains that the reason it is obviously flawed "is not immediately clear." For that reason, I am not sure why Rae maintains that it is obvious that the reasoning is flawed when he states two sentences after this that-to paraphrase-it is not obvious.

Then Rae acknowledges that "it is not at all clear what the relationship is between relevant [parts and whole]." If the part-whole relation is not well understood, this seems to indicate that mereological and compositional nihilism is an (obvious) alternative to material constitution, which is a theory with ancient metaphysical roots rather than modern scientific foundations, and which relies on a metaphysical relationship that is not even known to be non-paradoxical (and thus not even useful). But Rae does not mention mereological nihilism as being an alternative theory that is ready to fill the philosophical void that compositional and mereological philosophy creates-which implies that, for Rae, mereological nihilism is for some undisclosed reason, not an option.

Rae's passage illustrates how contemporary philosophers are aware of the problems of composition, but they nevertheless reject these problems (i.e., they do not take them seriously), thus believing and asserting that there is composition, even though they have no clear account for it, and even though theories of composition lead to paradox and contradiction. By analogy, the situation is much like when Einstein discovered the Big Bang by discovering that his equations showed strong evidence that the universe is expanding, but surprisingly he rejected the idea of an expanding universe because he was prejudiced by the assumption and the belief that humans live in a steady state universe, which he had literally no evidence for. Later he called this the biggest blunder of his career, and what he discovered is now known as dark energy, which is the recently empirically verified process of cosmic expansion of the universe.

\subsection{Article overview}

One of the primary objectives I have in this article is, in Sects. 3 and 4, to put forth a number of novel arguments, but specifically the following two: (i) a novel

\footnotetext{
${ }^{71}$ Rae (1997, xv-xvi). This passage is at the beginning of Rae's anthology on material composition. In that anthology he maintains there are essays that (allegedly) solve some or all of the problems of material constitution (xvi). Mereological nihilism is aimed at undercutting any such (alleged) solutions by showing that the relations needed for material constitution needed are in fact impossible, and thus no supposed solution(s) to the problem(s) of material composition and constitution can get started.
} 
argument for the impossibility of composite objects, and (ii) a novel argument for the impossibility of Democritean atoms (which are extended quantum atoms [explained below]). But it will be shown in Sect. 4 that (ii) is just an additional means of showing that there are no composite objects, distinct from the way that (i) shows there are no composite objects. (ii) also has to do with showing why there are no objects that can connect or touch any other objects, and thus no objects can accumulate into any composite object, as if to act as a part of a mereological whole. There are two ways composition is described by physicists, philosophers, and anybody else: parts contact (touch, attach, link, abut, adhere, etc.) to one another and thereby accumulate into composites, and parts connect (relate, interrelate, etc.) to one another and thereby accumulate into composites. With the former, parts directly interface, but they need not with the latter. With the aforementioned arguments in Sects. 3 and 4, I will show why these accounts are impossible, and instead why they only lead us to the conclusion that there are no composite objects. If there is no connecting or contacting at the quantum scale, then there is no contacting or connecting in macroscopic reality either, and this indicates that material constitution, which requires contacting and/or connecting of items, is impossible (and anti-reductionism cannot save the non-nihilists from this conclusion).

In Sects. 2, 6, and 7, I discuss several ways that I-empirical quantum physics provides evidence for mereological nihilism. I will point out that mereological nihilism may actually provide a clearer way, and a non-metaphysical way, to understand the data and experimental results of quantum physics than those we have been given by physicists. I will also discuss in detail in what ways that the many interpretations of the experimental data of physics are metaphysical, how the argumentation for mereological nihilism not only attacks compositional and mereological philosophies but also attacks the metaphysical constructions of the quantum physicists in their famous interpretation models of quantum mechanics (for example, the extended metaphysical/invisible waves believed to exist as an aspect of the wave-particle duality equation cannot exist given the reasoning against the existence of extended objects that I will present in Sects. 3 and 4), and what the quantum atomistic reality is like in light of the philosophy of mereological nihilism (this largely consists of giving an account of the I-empirical results of quantum studies). In Sects. 7 and 8 I will discuss the attacks against mereological nihilism that non-nihilistic philosophers (and physicists) have presented. We will see at various points in this article that the only substantial attack actually given against mereological nihilism is that mereological nihilism is in disagreement with ordinary empirical experience, and we will see why this is in fact not a good attack against mereological nihilism. In Sect. 7, I discuss the obvious point that if mereological nihilism is correct theory, then mereological nihilism shows that ordinary human perception is utterly flawed. This may be considered a problem by the vast majority of philosophers, who, of course, do not reject most or all information of empirical experience and empirical knowledge, but mereological nihilism appears to be in agreement with quantum mechanical theories-the theories that describe the smallest building blocks scientists have discovered-so there is reason to reject ordinary empirical evidence. I will show throughout this article, and often in explicit detail, why ordinary empirical experience is inaccurate and needs to be rejected. I will also discuss what I see as the only two possible theories of consciousness if mereological nihilism is a correct theory: Colin McGinn's cognitive closure, or a specific variety of quantum consciousness. Both of these theories of consciousness 
involve an extreme form of conceptualism, ${ }^{72}$ and both of these theories are unsatisfactory, as I will explain. In Sect. 2, I next discuss several important preliminary issues that need to be covered before a proper analysis of mereological nihilism can be given. Although it is nearly impossible to give a terse summary of this article in this introduction, the following passage from the University of Michigan physicist, Gordon Kane, can however serve to give some basis to the thesis of mereological nihilism:

The picture of the electromagnetic force that emerges is that electrons, and any particles that have electric charge, interact by exchanging photons. The photons can carry energy between electrons; two electrons can scatter off one another by exchanging a photon; and an electron and a proton bind by exchanging many photons, which provide an attractive force that keeps the electron and proton connected in a stable object, a hydrogen atom. All the forces work in a similar way. ${ }^{73}$

This passage shows how quantum reality does not involve interrelated, interconnected, or contacting quantum abstract atoms - there are no continuous (unbroken) fields, forces, relations, or topological connections between quantum atoms, and interaction is not clearly known to involve contact between particles (but is rather through exchange of particle and other non-contacting, non-relating means, as I will discuss). Kane's use of the word "connected" in the penultimate sentence of his passage can be seen from the sentences before to be referring to unconnected, unattached, non-contacting quantum abstract atoms. If this is the case, and if eliminativism (anti-antireductionism, anti-emergentism) can be shown to be correct, as I will in Sects. 3 and 4, then reality does not involve anything but unattached, unconnected quantum abstract atoms.

I am sure that critics of mereological nihilism and of this article will accuse me of absurdity since I argue for mereological nihilism with words like "section," as I did in the last sentence of the last paragraph, and which is just one of many examples of how I am obviously using part-whole talk in this article. But if I am to write an article

\footnotetext{
72 By conceptualism I mean that empirical experiences (representations) are mental fabrications, and all the experiences of colorfulness and extension humans have with their ordinary empirical experience about the external world are illusions, since they are based on erroneous construction of mental representations.

Conceptualism is typically defined as being halfway between nominalism and realism, where universals exist but only as mental contents, and they have no external (mind-independent) or substantial reality. Concepts have an ideal value, and they do not have a real value (non-ideal or mind-independent value). Since the term "conceptualism" is not used too often in philosophy anymore since the theory has not been in vogue, I will give passages from two other philosophers who discuss the theory. Doing this will help to ensure readers of what I am discussing when I use the word "conceptualism." First, Quentin Smith writes:
}

Conceptualism with respect to propositions is the theory that it is necessarily the case that propositions are effects of mental causes. It is necessarily true that for any $\mathrm{x}$, if $\mathrm{x}$ is a proposition, then $\mathrm{x}$ is an effect of some prepositional attitude. It may be said that the conceiving of a proposition (which may be taken as an element common to all prepositional attitude(s) is what sustains the proposition in existence, such that the proposition exists by virtue of this conceiving. (Smith 1984, 38-39)

And Jeffrey Brower writes: "Conceptualism is the view that there isn't anything in extramental reality corresponding to any of our relational concepts." $(2002,230)$.

73 Kane $(2000,18)$.

望 Springer 
on mereological nihilism and on the nihilism of quantum abstract atomism, the only language available for me to use to communicate the philosophy to other people is a part-whole, non-quantum-oriented language, which is based on ordinary empirical experience and on practical usage, not on philosophical and scientific precision. Minds that experience in the ordinary empirical mode tend to see beaches rather than grains of sand, shirts rather than strands of cotton, lawns rather than individual blades of grass, skin rather than skin cells, and surfaces and colors rather than quantum abstract atoms. I will explain at other places in this article that this is a selective mode of experiencing based on eliminating chaos and being overwhelmed by ordinary empirical experience, and not on philosophical precision. ${ }^{74}$ Ordinary empirical experience apparently and inadvertently tends to often forget that selectiveness in perceiving is going on, and, for example, it may be forgotten that when looking at a skin that the skin is cells. The mistake is typically made where it is believed that the primary things that exist are those that are experienced via the selective perceiving (skin rather than cells, beaches rather than individual sand grains). I do not see how to avoid the conclusion that language is based not on scientific and philosophical precision, but rather on the imprecision due to its being oriented toward ordinary empirical experiences and to the customs of ordinary empirical experience. The Inuit have dozens of words for snow, whereas in English we have one. The Amish do not use the word "Zen" nor have a concept like the Tao. The Advaita Vedantins do not use the phrase "zero-point energy" and the astrophysicists do not use the concept of Brahman. These are a few of countless examples that could be given that seem to point to the (apparently obvious) fact that language is utilitarian-as philosophers before me have famously pointed out. We only need disciplines like science in order to help correct for the crudeness of ordinary empirical experience. If ordinary empirical experience was sharp and precise, why would there be a need for scientific thinking, and logical argumentation? I am therefore forced to use the ordinary empirically based, surface- and color-based, mereology-based language (in my case, English) in order that others will understand and in order to communicate the philosophy of mereological nihilism. This is of course what quantum physicists also do, but where they have often "humanized" (Kane) the discourse more than I will (and their doing so has led to serious confusion, even among themselves, as I will explain, and as I will try to avoid in this article.)

\footnotetext{
74 I am using the word "chaos" here in the same way that William James tended to use it, such as in the following passage (which is a passage that is only about DIV2 reality) that helps us to understand the way that ordinary empirical experience is so limited in scope:
}

The world's contents are given to each of us in an order so foreign to our subjective interests that we can hardly by an effort of the imagination picture to ourselves what it is like. We have to break that order altogether-and by picking out from it the items which concern us, and connecting them with others far away, which we say "belong" with them, we are able to make our definite threads of sequence and tendency; ... and to enjoy simplicity and harmony in place of what was chaos. Is not the sum of your actual experience taken at this moment and impartiality added together an utter chaos? The strains of my voice, the lights and shades inside the room and out, the murmur of the wind, the ticking of the clock, the various organic feelings you may happen individually to posses, do these make a whole at all? Is it not the only condition of your mental sanity in the midst of them that most of them are should become non-existent for you, and that a few others... rational... The real world [DIV2 world] as it is given objective at this moment is the sum total of all its being and events now. But can we think of such a sum? Can we realize for an instant what a cross-section of all existence at a definite point of time would be? (James 1992, 545-546). 


\section{Problems with the empirical and the ramifications of mereological nihilism}

Before going on to my specific arguments against mereology, mereotopology, topology, and any theory of material constitution, in this section I will begin to shed light on what the ramifications are of a quantum mereological nihilism being the correct theory of reality, and I will more specifically clarify why ordinary empiricalmaterial reality (but not I-empirical reality) is paradoxical. I will specifically show how philosophers ubiquitously ignore and suppress the evidence that shows that ordinary empirical-material reality does not exist, and that quantum mereological nihilism is the correct theory. Also, I will discuss how there are different types of mereological nihilists, and how some of them may not be pure and thoroughgoing nihilists who maintain that only quantum philosophical atoms exist.

\subsection{Widespread doubts about mereology}

Philosophers commonly believe that parts and wholes exist, and that this is so obvious that there need not be any concern about the question of whether or not there are any in the first place: the existence of parts and wholes is so commonsensical that no debate over whether or not parts and wholes exist is needed. For example, in their book on substance, consider how Hoffman and Rosenkrantz discuss the relation of part to whole, as if it is a main intuitive aspect of reality: "According to a key commonsense intuition, there is a relation that unites parts which compose a compound physical object." $" 75$ In this way, philosophers (especially those who are not specialists in mereology, the philosophy of composition and material constitution, or mereological nihilism) often freely refer to parts and wholes when doing their philosophical work, as if there is no question that there are things that are parts, and that there are things that are wholes. But specialists in mereology,

\footnotetext{
75 Hoffman and Rosenkrantz (1997, 73).
}

In their book, Hoffman and Rosenkrantz base the existence of part-whole relations solely on this demand that they are commonsensical. Even when addressing important attacks by mereological nihilists they nevertheless only fall back on this one claim of commonsense as a supposed counter attack.

Many philosophers who discuss mereology and composition (or, compounds, as Hoffman and Rosenckrantz refer to them) maintain that mereological relations perhaps involve some problems but (paradoxically) they are still commonsensical (for example, see Simons 1987, 10-11, where he maintains that part-whole relations are utterly commonsensical, even though his entire book Parts is based on the discussion of serious problems with these relations.) Hoffman and Rosenkrantz base all their discussion on mereological relations from the alleged commonsense nature of them, seemingly believing that's enough for their existence-despite their problems - and thus enough to not entertain mereological nihilism. For example, on pp. 181-182 of 1997 they object to van Inwagen's non-mereological metaphysics by merely passing over van Inwagen's pointing out of problems to do with mereological relations, instead letting their trust in their commonsensicality guide them. Also, on page 79 they maintain that they are willing to usurp some results of science which are "well confirmed" (78) and which point toward mereological nihilism in order to "defend the commonsense view [of composition]" (79). This is a good example of philosophers deliberately rejecting scientific findings (and even simple logical findings) - and even admitting that they carry out such a rejection-in favor of commonsense (where "commonsense" is a very crude and unrefined commonsense-i.e., it is a plebian DIV2-empiricism that is not honed by science). I will discuss all these issues in detail in Sect. 8, where I will show other specific examples of philosophers rejecting science and logic, and admitting that they are basing their philosophy only on a plebian prephilosophical intuition and commonsense, as Hoffman and Rosenkrantz appear to be doing here. 
mereotopology, topology, or the philosophy of material composition very commonly admit and put emphasis on the serious problems involved with how any objects can be composite. Consider what Simons writes at the very opening of his widely discussed book, Parts: "The principle theme of this book is that despite their formal elegance, these [mereological] theories leave much to be desired as general theories of part and whole."76 From what I can tell, contemporary philosophers who specifically write about material constitution and mereology emphasize the utter commonsensicality of the fact that there are parts and wholes (Simons does this on pages 10-11 of Parts), but also put significant emphasis in their research on the problems and inadequacies of the theories of composite objects. (Of the aforementioned theories of material composition, mereology and topology seems to be the most widely criticized regarding this issue, mereotopology the least.) It is as if they want to say: "We can see or intuit it, but we can't tell you how it works," which is the hallmark of ordinary empirical reality's most basic issues: motion, time, space, change, extension, material constitution, etc. Consider a passage from Markosian, where he goes as far as to admit that none of the theories of material constitution are sufficient:

Recently, a growing body of philosophical literature has concerned itself with questions about the nature of composition... The main question [which is called the SCQ] that has been raised about composition is roughly this: Under what circumstances does something compose, or add up to, or form, a single object? It turns out that it is surprisingly difficult to give a satisfactory answer to this question that accords with standard, pre-philosophical intuitions about the universe's composite objects... No one has yet defended a view in response to the above question about composition that is consistent with standard, pre-philosophical intuitions about the universe's composite objects. ${ }^{77}$ (My emphasis)

Markosian also writes that "... all of the... leading responses to SCQ are unacceptable.",78

There has been enough difficulty to do with the theories of mereology that a new theory, the aforementioned mereotopology theory, has been developed, ${ }^{79}$ and is the result of mixing mereology and topology in order to replace mereological theories. Casati and Varzi write:

How much of the spatial universe can be grasped and described by means of purely mereological notions? We argue that one cannot go very far. In our view (and this is a view we share with others...), a purely mereological outlook is too

\footnotetext{
76 Simons $(1987,5)$.

77 Markosian (1998b, 211). van Inwagen introduces the SCQ on page 20 of 1990.

78 Ibid., 214-215. Markosian writes this in trying to combat the existing theories of composition (universalism, etc.), and he argues all theories "are unacceptable" so as to try to make way for his new theory he presents in Markosian (1998b).

79 See Grupp (2005b, 91-93, 2005b, d, 2006b) for attacks on mereotopological theories.
} 
restrictive. At the very least, one needs to integrate it with concepts and principles of a topological nature. ${ }^{80}$

In this article I will show, as I have with different arguments elsewhere, that mereotopology cannot save or make coherent the philosophy of material constitution, as some philosophers allege it can. This is because mereotopology suffers from the same flaw I will show that topology and mereology suffer from: topology, mereology, and mereotopology all depend on invisible (not I-empirical) metaphysical relations between items, and/or on there being contact between items, but I will show all connections, touching, and contacting between items is impossible, and thus mereotopology (just like mereology and topology) is impossible, regardless of what one might believe reality is like based on their ordinary empirical information about reality. I will argue in this article that the difficulty with mereology and composition, and the reason why (in Markosian's words) "[n]o one has defended a view..." of it is because there cannot be any composition of any sort, and it is merely a product of the concepts of the mind.

\subsection{Antinomies and infinite divisibility}

Ubiquitously, scientists and philosophers tell us that ordinary empirical reality is coherent, and that if we find a problem in our analysis of it, then there is a problem with our analysis, not with nature. The evidence of this article shows that this is incorrect. It has been discussed for thousands of years by philosophers that if we merely attempt to describe the very simplest issues about ordinary empirical reality-motion, change, time, space, contacting or touching, extension, and so on-we arrive at apparently insurmountable paradox and contradiction. This would imply that what is known in philosophy as a reductio argument (if I accurately describe $\mathrm{x}$, but in doing so arrive at a contradiction, then I know that not-x is correct) could be given to show the non-existence of ordinary empirical reality: if we describe the most basic features of ordinary empirical reality, but arrive at contradiction, then ordinary empirical reality does not exist. This is what occurs with ordinary empirical reality: if we attempt to describe its most basic features, we arrive at contradiction, and its inverse-not ordinary empirical reality-must be real, and the best candidate for what "not empirical reality" could denote is the I-empirical quantum atomic reality. Interestingly, this article demonstrates that current science (quantum mechanics) also brings us to the came conclusion. Humans have a plethora of empirical experiences, but those experiences contradict one another. For example, if I probe into the smooth surface of a piece of paper in order to find out what the paper is made of, I arrive at point-particles that are surfaceless - which is a contradiction, since sizeless objects (points) cannot compose objects with size (surfaces). These empirical experiences contradict, and we are forced to make a decision on which of

${ }^{80}$ Casati and Varzi $(1999,4)$. They further add on page 10 that

...things are not as straightforward as this [it] might sound. Even if the spatial structure of an object depends crucially on the relations among its parts, it does not follow that mereology... affords the right way to investigate such relations... [A] purely mereological outlook may be utterly inadequately for the purpose of spatial representation, and it may be necessarily to integrate mereology with concepts and principles of various other sorts... [W] e will argue that some such concepts and principles are of a topological nature. The move from mereology to mereotopology... is a crucial first step in the direction of a good theory of spatial representation.

Springer 
the two opposed experiences is correct: the experiences of the extended empirical paper, or the experiences of I-empirical point-particles. In this article I show that only the I-empirical experiences of quantum abstract atoms involve non-contradiction (and thus are the experiences of real objects), and ordinary empirical objects are impossible (involve contradiction) and thus are not real. In other words, visible objects, objects with a surface and a color, do not exist. (To show this point, I will have to show that physicists are incorrect when they assert that quantum reality involves quantum paradox. I do this in Sect. 6.)

An interesting outcome of mereological nihilism is that the paradoxes and absurdities involving the ordinary empirical level are avoided: mereological nihilism avoids the antinomies, absurdities, paradoxes, and problems that have been widely acknowledged and discussed by philosophers. For the mereological nihilist, they are not problems, paradoxes, and so on, but rather are expected outcomes of the attempt to describe impossible items (composite items) that cannot exist, similar to the difficulties that would arise if one attempted to describe a round square. When one accepts that reality really is the way ordinary empirical experience indicates, disaster however strikes when a mere attempt is made to describe the most basic issues about that reality-such as how macroscopic objects move, how they change, how they persist through time, what they are made of, and so on. The paradoxes and absurdities are the result of attempting to describe all of reality in a way that conforms to this ordinary (unrefined) empirical experience. (In Sect. 8.1 below I will show how ordinary empirical experience that is not scientifically oriented is "unrefined" experience, meaning that it is involves conflicts with scientific empirical information.) There is a substantial list of rather implacable and widely known dilemmas waiting for the philosopher who accepts as real the surfaces and colors of their ordinary empirical experiencing, but there is no such list waiting for the mereological nihilist philosopher, who avoids all these problems. Mere attempt at description of any composite and/or ordinary empirical object leads to paradox that is revealed by the deconstruction of the object that the description involves. (In other words, analysis of any composite object, including space, time, and so on, shows that the object does not make sense.) Only composite objects, which involve complexities, can be deconstructed and shown to be absurd. But since non-composite objects (quantum abstract atoms) do not involve complexities, they cannot be deconstructed to reveal any paradoxes and absurdities. This is because only objects that involve complexities (distinctions, contrasts, etc.) involve paradox and absurdity. The simplest example is this one: for any composite object, it must be explained how it can be made of other objects (the composite's parts) that are not identical to it (which is an absurdity, since it involves the idea that composite $\mathrm{C}$ is self-identical, but is composed of other items which are not C). No such difficulties arise with noncomposites, which are unstructured, complexity-free quantum abstract atoms, which cannot be deconstructed (and which are ultimately almost, but perhaps not quite, ineffable). Mereological nihilist reality is utterly simple, and nearly all of its descriptions are negative descriptions (objects do not have size, do not involve change [see Sect. 4.5], do not have color, etc.).

It is widely known by philosophers that theories of matter (matter as was defined above and as will be further discussed in 2.4) involve antimonies. Even though philosophers typically pass over these, as if they don't put an end to the ideas of time, change, identity, motion, material constitution and so on, I see no reason why these problems do not give reason to doubt the existence of ordinary empirical reality and 
its composite matter. Van Inwagen, in his somewhat nihilistic book, Material Beings, also takes this as a reason to espouse mereological nihilism:

That there are deep and intractable metaphysical problems about material objects - even paradigmatic ones-is evident from the antinomies and paradoxes involving material objects. The best known of these are about artifacts, the puzzle of the ship of theseus being the best known of all... In this book, I shall present and defend a theory about the nature of material things that takes seriously paradoxical features of their unity and persistence. If this theory is correct, the nature of material things is radically unlike what most philosophers suppose it to be... I shall try to show that the "deep and intractable" metaphysical problems about the unity and persistence of material things have simple and intellectually satisfying solutions if my proposals are correct. ${ }^{81}$

Philosophers very often accept and put their trust in empirical reality, unquestioningly believing it really is how we believe we perceive it (such as having surfaces and colors), and then with this dubious assumption, they carry out and construct their philosophical theories and do their philosophical work-work which is based on, and built up out of, paradox- and antinomy-riddled ordinary empirical reality. Quantum mereological nihilism avoids this and thus one might imagine that mereological nihilism would almost unquestionably be the philosophy of the day. But surprisingly, rather than considering mereological nihilism as the welcome savior from a philosophy of absurdity, almost always mereological nihilism is instead labeled absurd or radical (where no argument follows as to why this it is), and no recognition follows where it is revealed that mereological nihilism appears the coherent philosophy, and the philosophies of orindary empirical reality (the philosophy of space, of time, of ordinary objects, and so on) are actually the antinomy-, problem-, and paradoxriddled philosophies. I will next consider an example of such ad hominem to do with the philosophy of time (time is a composite object, consisting of at least the parts past, present, and future) here to illustrate this. (So far I have been discussing mereological nihilism about space and matter, but mereological nihilism about time-which is called presentism ${ }^{82}$-also should be mentioned). The example has to do with one of the most famous paradoxes or problems - the problem of the self-identity of any apparently persisting object through time or change (which is impossible since an object cannot remain itself (the same) if it changes). Rather than mereological nihilism being considered the (perhaps obvious) solution to this problem (a solution that would amount to a denial that there is time or change), instead, and, surprisingly, it is merely demanded that such nihilism is "radical." Consider the following passage from the widely read Stanford Internet Encyclopedia of Philosophy:

\footnotetext{
${ }^{81}$ Van Inwagen (1990, 17-18).

82 Presentism rejects persistence (Zimmerman 1998, 213), rejects past and future, and thus there is just one irreducible unchanging point of presentness. But if it is unchanging and does not have any past or future parts, then it does not involve persistence, endurance, change, or time flow. Thus, presentism leads to the position that time does not exist. These ideas are discussed in much more detail in Grupp (2005a, c), which both deal with mereological nihilism about time (no parts of time means there is no time at all). In this article I focus much more on nihilism about space and matter than I do on nihilism about time in this article since I exhaustingly covered nihilism about time in other articles.
} 
Temporal-parts accounts of change seem OK, but can endurantists come up with anything equally good or better? How can one and the same thing have different properties at different times? One radical answer is presentism, the view that only present objects, events and states exist: past and future do not exist. Presentism seems to dissolve the problem of change: since only the overripe banana is present, its unripe state does not exist, so there's nothing to worry about... But unless there are independent reasons for accepting presentism..., it seems like a drastic response to the problem of change. ${ }^{83}$ (Emphasis added.)

Although this passage is short, nevertheless, twice in this passage, emphasized by the italicized words, is the urging and the attempt to persuade that the presentist position is radical and drastic. No argument is given however for why this is the case. This is the standard way nihilism is dealt with: although it solves perhaps nearly all of the many famous paradoxes and problems of philosophy thus (finally) making philosophy consistent, and although it fully aligns philosophy with the most progressive area of science (quantum mechanics), it is instead rejected without argument nor evidence, but rather with unphilosophical ad hominem "reasoning." Since there are no clearly outlined claims made regarding why presentism is drastic and radical, using these words takes the appearance of being an attack that is, as I mentioned, the informal fallacy of ad hominem-much like when Camus' Meursault was found guilty of murder largely for sleeping through his mother's funeral, rather than for killing the Arab. And it is no secret among professional philosophers that mereological nihilism avoids the antinomies and paradoxes of empirical reality. In a recent article, Markosian attempts to refute nihilism (his attack is discussed elsewhere in this article), but before doing that, he comments on the obvious strengths of mereological nihilism, such as that it avoids the widely discussed absurdities and paradoxes just mentioned about the empirical and composite objects. He writes: "Another virtue of Nihilism is that one who subscribes to it need not worry about any of the traditional puzzles concerning the identity of composite objects." 84

Certainly some readers will be puzzled as to the implication here that it is only the empirical level of reality that involves paradox, not the quantum level. It is widely held that the domain of the quantum is a domain of fantastical paradox. But most of these alleged paradoxes have to do with physicists creating them for us, merely informing us, rather than discoverying for us, that quantum reality involves impossible states of affairs "that exist." Countless paradoxes and mysteries emerge in science and philosophy (e.g., why do galaxies appear to have much more matter than they exhibit [this is the dark matter problem]), and there are many in physics. But for some reason when the paradoxes involve a few issues to do with the fundametnal particles, we are informed by physicists that they actually indicate real facts about reality as being nonlogical-rather than mere problems with interpretation of data, and rather than mere need for further investigation into the mysteries of quantum reality. The equations physcists used to discover and formulate quantum reality do not appear to be absurd, so it is puzzling as to why the reality they lead us to is alleged to be. There are no arguments for why we must accept the paradoxes, and certainly not all physicists do. What exist are a few famous and, frankly, often even quite hostile physicists (such as Feynman) who have literally outspokenly castigated anyone (especially philosophers)

${ }^{83}$ Hawley (2004), section 3.
${ }^{84}$ Markosian (1998b, 219). 
that does not merely accept that nature is describable as absurd at the fundamental level. Indeed it may be paradoxical at that level, but that it is would need to be proven, not asserted: lack of data does not reveal paradox, but rather only lack of data. Dark matter will appear mysterious until we discovery what it is. The transfer and passing along of traits by reproducing animals appeared irreducibly mysterious until humans discovered genetics - now we have genetic engineering.

If one looks at the alleged paradoxes of quantum physics, we can see that they are certainly not anything more than shoddy non-philosophical theory-building originating from merely a few very famous quantum physicists, as I will show in Sect. 6. My use of the word "shoddy" may appear overblown, but these paradoxes are only the result of (a few) physicists doing metaphysics. For example, it is now popular for physicists to tell us that electrons are "waves" (probability waves) due to various quantum mechanical findings. But no physicists has ever observed a wave in the way we can observe a water wave (where we can see the extended wave waving up and down, etc.). (The waves are only "observed" as highly contrived computer images.) Physicists only witness point particles, and thus the waves are purely metaphysical-advocated by a few physicsts after Bohr to demand that nature is paradoxical, rather than merely acknowledging that there is a mystery in their data that could be figured out with further research. But since it is this non-scientific metaphysics that leads to their conclusion that there are so-called probability waves, it seems fair to label the theory-building as "shoddy:" either a product of poor metaphysical theorybuilding by physicists (poor because of the aforementioned desire to specifically make the theories paradoxes), as just described, or are a product of not looking at the experimental data closely. Stenger writes:

Particles and fields have become intertwined in the usual expression of quantum physics. Photons and electrons are said to exhibit properties of both particles and fields, appearing localized and distributed, discrete and continuous. This is usually called wave-particle duality. You will often hear that something is a particle or a wave, depending on what you decide to measure. I have said this many times myself in the classroom, parroting what I heard in class and read in textbooks when I was a students. But in recent years. I have recognized what a dangerously misleading and indeed incorrect statement this is... In fact, no experiment provides data that speak against the fundamental particulate nature of light. Whatever you decide to measure about light, if you do so with sufficiently sensitive photoelectrons you will always detect something localized and discrete-a photon. ${ }^{85}$

\subsection{Types of nihilists}

There are a few varieties of mereological nihilism, and they differ by the ways in which a mereological nihilist discusses consciousness and phenomenal states, and human persons. I list three below, the first of which is the position of the contemporary nihilists other than myself:

I. Impure physicalistic mereological nihilism. This has also been called quasi-mereological nihilism, and it is a position involving two primary features.

\footnotetext{
85 Stenger $(2000,110)$.

iㅡ. Springer
} 
Ia. Atoms are physical and they interact in some way (they contact or are interconnected), and

Ib. There are some objects-namely the minds or bodies of some living organisms - that do have parts, and thus are mereological wholes that are admitted into this impure mereological nihilism (hence the word "impure," since there are some composites), but for the most part, of the items that exist, it is the minority of them that have parts. There are questions as to whether or not the proponents of this position have specifically described how minds and/or bodies can be composite items while other items cannot. ${ }^{86}$

II. Dualist mereological nihilism. This has similarities to position I except for the following difference: minds are not reducible to atoms, and minds are ineffable non-composites (which I will call McGinn minds). (There could be cases where a nihilist endorses both I and II.) According to this position, it is unclear what minds are made of, but it can be made clear that they are not reducible to the quantum abstract atoms that are identical to the rest of reality. McGinn minds can only be made of some undiscovered non-particulate, non-physical "mind-stuff." There is no reason to argue that the mind is either partless or composite. Simply put, this exotic "mind-stuff" would be entirely unknown "stuff," which no theory could ever describe.

III. Quantum panpsychistic mereological nihilism (QPMN). This position could also be called pure mereological nihilism. Non-nihilistically, both I and II above involve making a distinction between quantum abstract atoms and personhood (human bodies or states of consciousness), but QPMN does not. On this pure mereological nihilist view, there is only one sort of "stuff," which is the indistinguishable quantum abstract atoms (which is the basis of a pure mereological nilihilsm), and since consciousness also exists, then those structureless quantum abstract atoms can only be identical to consciousness. This is an panpsychistic theory, since it indicates that consciousness is everywhere quantum abstract atoms are, which is to say that consciousness is reality. ${ }^{87}$

In this article I will not formulate an argument as to which of these is correct, but I will discuss in Sect. 9 why III appears to be the best option. For pure mereological nihilism that is non-metaphysical and that is scientifically founded, it seems that III is the only option.

The problem with what is being discussed here is that this discussion involves the philosophy of mind or consciousness, but it is unclear how the mind or consciousness exists if mereological nihilism is correct, due to the fact that it is unclear how mind or consciousness is identical to quantum atoms. This might seem to be a weakness of this article (many philosophers have told me they believe it is), but that claim is flatly false, for the following reason. This problem just described in this paragraph to do

\footnotetext{
${ }^{86}$ For example, van Inwagen points out why we should reject composite objects, but does not explain how a human body can be one.

${ }^{87}$ Some readers might believe that it makes more sense to assert that according to the QPMN position, that consciousness is identical to the universe. But as is shown in this article, universes do not exist, unless they are considered to be denoted by the following statement: "unstructured unconnected unattached particles." But that does not seem to be the way that universes are typically described, and instead, the ordinary conception of what a universe is, is a mereological whole-and thus "universe" denotes nothing whatsoever, on the ordinary usage of the word. The information in this footnote shows how mereological nihilism is similar to the theory called acosmism.
} 
with mereological nihilism is merely the same problem that is found in the other areas of philosophy, and in the philosophies of mind or consciousness, which goes as follows: How do fundamental items (that appear to be non-mental "stuff") support, connect to, or give rise to our "Technicolor phenomenology," as McGinn puts it? Since this is a problem for all of philosophy, I will in no way imagine that it is only a problem for mereological nihilism. It is safe to say that nobody has discovered what the building blocks of phenomenological "mind-stuff" are, and if there are any, how they give rise to phenomenological "stuff." These are unsolved problems of philosophy, and are no more of a problem for mereological nihilism than they are to non-reductive materialism, idealism, and so forth.

As for type-I, the impure-mereological nihilists, one variety, endorsed by van Inwagen, involves the position that there is one type of object that has parts, namely, human persons (by which van Inwagen apparently means humans' physical bodies). In van Inwagen's impure-nihilism there are no other objects with parts, and all other objects that we believe to be composite-chairs, trees, etc.- therefore do not exist. Rather, other than persons, which are composites, there are only partless fundamental particles. For example, van Inwagen and other impure physicalist mereological nihilists, as we are calling them, maintain that our language and our sense information is not totally mistaken since when we refer to things like chairs and galaxies, we are referring to particles that are arranged in a certain way, and thus when we say "chair", we are not referring to one item, but rather we are referring to a collection or network of quantum particles in a specific "arrangement". Consider this diagram, which illustrates impure physicalist mereological nihilism:

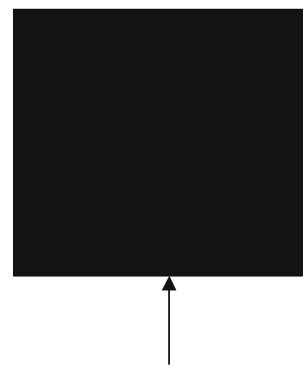

This is how humans observe things empirically: there appear in the consciousness of that observer items with a color, solidity and smoothness.

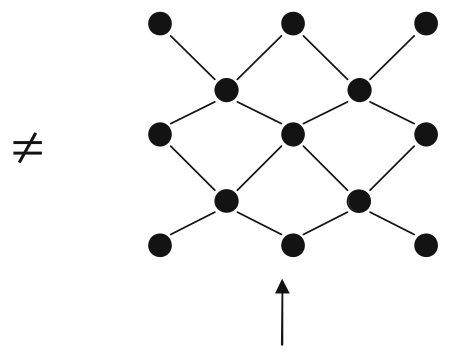

This is more in accord with how things really are at the most fundamental scale, according to the impure mereological nihilists: there is not one thing, but many interconnected philosophical atoms which, we are told by impure physicalistic nihilists do not make up single objects. Much of this article is devoted to showing that there are no such interconnections.

Below I will argue that impure physicalist mereological nihilism is an impossible theory and should be rejected in favor of a pure mereological nihilism (a nihilism not involving any parts or any interconnections of any sort, as the phrase "mereological nihilism" most faultlessly denotes). I will come to this conclusion because I find that there are no such relations of arrangement or relations of part-to-whole. Even if there were such relations between atoms, it is unclear why they would not allow atoms to give rise to mereological wholes, and thus type-I nihilists would not be nihilists at all. The reason that interconnected atoms seem to give rise to mereological wholes is because the touching and contacting between any two objects 
(such as an atom and a relation) involves a shared part between the items that touch or contact. ${ }^{88}$ But if there is a shared part, then the objects that share the part (in this case, a relation and an atom) form a fusion and it would no longer be the case that they are entirely distinct items. Therefore, if mereological nihilism is a correct theory, there are no interconnected atoms (or any interconnected items at all), which we will find out is the case in Sect. 4. It is not clear how atoms could have parts to share and thus it appears dubious to imagine that an extended atom can touch or contact anything else, such as another atom, or such as "touching" (linking to) another relation or an instantiation tie of a relation that bridges ${ }^{89}$ the atom and the relation. This is one reason why atoms cannot touch one another, and I will point out other reasons why atoms do not touch one another in Sect. 4.

\subsection{DIV1 and DIV2: two ways of experiencing}

Reality as it is ordinarily believed to be is viewed in terms of levels, or strata: the chemical level is made up of the particles of physics, biological organisms are made of chemicals, and so on. If, as Schaffer writes, "the central connotation of the 'levels'

\footnotetext{
${ }^{88}$ See Grupp $(2003,2004 a$, b) for details on why relations (polyadic properties) cannot be shared by items since they cannot contact the items they allegedly interconnect. But ignoring the issues in those articles, and discussing why networks of interrelated items can only involve shared parts (and thus can only be mereological holes), consider that contact and touching between objects involves the relation of collocation between surface points (which is a relation I show does not exist in Sect. 4). Consider a passage from Chisholm:
}

Let us recall an ancient problem. "Consider two discrete physical bodies thought to be continuous with each other; the east side of body A, say, is continuous with the west side of body B. How is this possible? Either (i) the eastmost part of A is in the same place as is the westmost part of B or (ii) no part of A occupies the same place as does any part of B. In the case of (i), we would have two discrete things in the same place. But this is impossible. In the case of (ii), since A and B occupy different places, there is a place between the place where $\mathrm{A}$ is and the place where B is. But if there is a place between A and B, then A and B are not continuous." (Chisholm 1989, 84)

Chisholm's passage indicates that it appears to be the case that there cannot be contact, abutting, or touching unless there are items that coincide. Collocation or coincidence also means that there is a shared part between collocated objects: "Two individuals overlap mereologically if and only if they have a part in common." (Simons 1987, 11) Chisholm does not go on to give answers to this "ancient problem". But Chisholm states that "it holds of things that are merely continuous with each other but which are not continuous with each other (for example, two blocks pushed together). A similar objection applies to the suggestion that, if two bodies are continuous with each other, then there is no space between them. Barry Smith has written about Chisholm's work in a passage that includes discussion of point-sized items in the problem of their touching and contacting:

Imagine... two perfect spheres at rest and in contact with each other. What happens at the point where they touch? Is there a last point $p_{1}$ that belongs to the first sphere and a first point $p_{2}$ that belongs to the second? ... [C]learly not, for then we should have to admit an indefinite number of further points between $p_{1}$ and $p_{2}$ and this would imply that the two spheres were not in contact after all... [T]o admit that the point where the two spheres touch belongs to neither of the two spheres seems to amount to the thesis that the two spheres do not touch at all. (Smith 1997, 535-536.)

In Sect. 4 I will show that contacting between any coinciding items (even point-sized ones) is impossible, and I will show that all contact is impossible. In pure mereological nihilism, there are no interrelated items, and no items that contact each other in any way.

${ }^{89}$ Loux uses the word "bridge" to denote the special instantiation tie (also called the exemplification tie) that ties a relation to its relata. See Loux $(1998,38-41)$. 
metaphor is that of... a mereological structure, ordered by a part-whole relation," 90 then the mereological and compositional nihilist can only conclude that there are no levels to reality. As discussed above, quantum particles are not directly observable (they cannot be observed directly from the ordinary empirical-material perspective). For that reason, I will explain in this subsection that the divisions of reality can be divided into two sorts: empirical-material, and quantum abstract atoms. I will call the empirical-material division 2 (abbreviated DIV2) and I will call the quantum abstract atoms division 1 (abbreviated DIV1). The following diagram illustrates these ideas of DIV1 and DIV2:

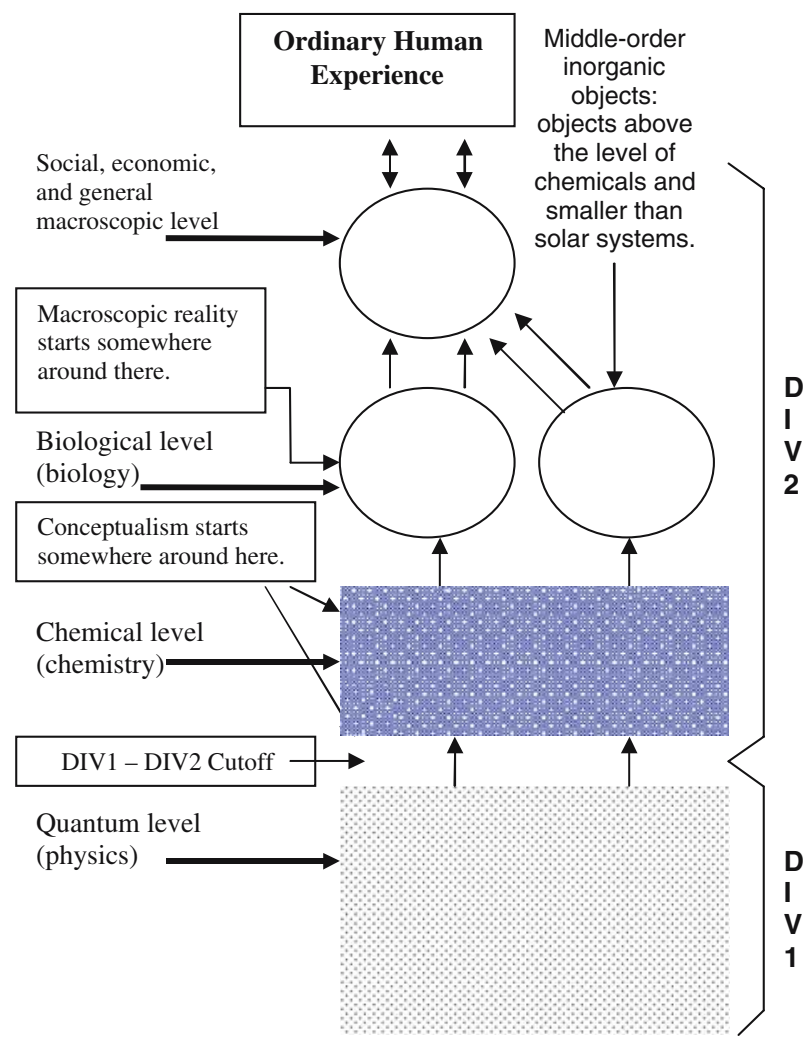

These divisions correspond to types of experience one can have. One can have either empirical experience of material things that have a surface and a color (DIV2 experience), or one can have I-empirical experience of the quantum atoms (DIV1 experience). DIV2 experience will occur through the apparatus of DIV1 experience, as I will discuss, but nevertheless, the objects involved in either of the two sorts of experience are the inverse of one another: one has surface and color, the other does not, etc. DIV2 consists of anything larger than the scientific atom (which, as

\footnotetext{
90 Schaffer (2003, 500).

Springer
} 
mentioned above, is distinct from the philosophical atom). And DIV1 consists of the quantum abstract atoms. ${ }^{91}$

Although DIV1 is typically considered to make up DIV2, according to the arguments of this article, DIV1 is real and DIV2 is merely a conceptual construction of consciousness. The characteristics of DIV2 objects and the rules that objects in DIV2 operate according to are the inverse (opposite) of characteristics of DIV1 objects: if a DIV2 object has property $\mathrm{P}$, then any DIV1 object has property $\sim \mathrm{P} .^{92,93}$ In the first words of his recent book, Omnés, a physicist, writes:

If we must rethink today the links between philosophy and science, it is because we are in the aftermath of a fracture. The most fundamental sciences, those dealing with space, time, and matter,... have broken out of the limits of common sense and traditional philosophy. ${ }^{94}$

\begin{abstract}
${ }^{91}$ It is unclear whether or not the scientific atom is of DIV2 or DIV1. It is "observed" through spectroscopy, and what is observed during the spectroscopy is a surface shape, viewable on the computer screen. But this surface is generated by the computer based on where the sensor tip is absorbed by the quantum atoms that allegedly make up the scientific atom. I will show below that electrons, for example, are not probability clouds, contrary to popular belief, and electrons can only be point particles (quantum atoms). So in spectroscopy, through absorption of the sensor's micro-tip by the scientists' atom's quantum atoms, the image of a surface is fabricated. For these reasons, the scientists' atom is not at all clearly a DIV2 extended item. But on the other hand, it is not a quantum atom since it is a composite that is alleged to exist. For these reasons, I do not know where the scientists' atom belongs, in DIV1 or DIV2. But my guess is that it goes in DIV2, and can merely be thought of as the illusion of a surface generated by quantum atoms.

92 I have found this an interesting and surprising characteristic of the differences between DIV1 and DIV2. The rule seems to hold in all cases except two: properties that DIV2 items have are always the negation of properties DIV1 items have except for the case of tiny motion and duration for particles. The data of quantum experimentation appears to reveal that DIV1 objects (quantum atoms) may have an extremely tiny duration.
\end{abstract}

${ }^{93}$ Actually, quantum abstract atoms are more likely propertyless items (see Grupp 2006a). Traditionally, this has not been specifically an issue to do with mereological nihilism, which has been considered to only require partlessness and the ramifications of a partless reality. Propertylessness has to do with a similar theory, blob theory (it is similar to blob theory since both theories involve a reality that does not involve wholes, structure, space, time, diversity or differences, and so on, and in a note above I discussed how the reasoning in Sect. 4 may reveal that mereological nihilism is actually identical to blob theory). Blob theory, and perhaps also mereological nihilism, would indicate that DIV1 is a propertyless realm, where accordingly DIV2 would consist of little more than aimless and illusory (i.e. non-representational) concept-making (i.e. property-making) of consciousness (assuming consciousness somehow exists in a mereological nihilist reality). I do not describe the DIV1 - DIV2 differences in this article in terms being propertyless (DIV1) and having properties (DIV2) for two reasons. (1) Discussing propertyless reality is impossible (that is, a blobby, propertyless reality is ineffable), since any sentence describing that reality would appear to be a description denoting properties it has (for example, here is a description of blob reality, it is noncausal, immaterial, propertyless, etc., but the words "non-causal," "immaterial," "propertyless" appear to pick out properties of DIV1 objects). So it is better to describe things in more familiar terms, with familiar language, just in order make the case for mereological nihilism and blob theory — which can only be done by using DIV2 language - rather than also attempting the difficult task of trying to make the case for the propertytlessness of the quantum abstract atoms. (2) Discussing the DIV1 - DIV2 difference as having properties that are the inverse of one another, as I have indicated I am doing in this article, basically achieves the same goals as if I were to attempt discuss reality in the difficult propertyless versus. not-propertyless manner-in either way of description (propertyless versus having properties, or having parts vs. not having parts), I am ultimately describing DIV1 to be entirely unlike DIV2, where it shares no similarities with DIV2.

94 Omnés (1999b, 3). 
Watson, another physicists, writes: "The subatomic world is a world where what physicists call 'classical rules,' applicable to objects large enough to be held in the palm of the hand, do not apply." 95 Pointing out the difference between the empirical and non-empirical nature of DIV2 versus DIV2, Omnés writes:

There is so wide a chasm between classical and quantum physics that one still marvels that it could be bridged. These two conceptions of the world are almost opposite in every respect. Classical physics relies directly on a reality that one can see and touch, and which one can act. It is basically causal, to the extreme of determinism. On the other hand, quantum physics deals with a world in accessible to our senses and our common sense, a world that can only be described by abstract mathematics. The most commonplace objects have a shadow of wave functions, simple quantities become matrices, operators, pure constructs of pure mathematics. In place of the excessive certainty of determinism, one is facing absolute randomness. Could there be a more drastic opposition $?^{96}$

The famous physicist Brian Greene writes:

[Our] day-to-day experiences are classical experiences; with a high degree of accuracy, they conform to the laws of physics set down by Newton more than three centuries ago. But of all the discoveries in physics during the last hundred years, quantum mechanics is far and away the most startling, since it undermines the whole conceptual schema of classical physics. ${ }^{97}$

And in another passage, consider what the physicist, Ford, writes:

...the physics of the past hundred years has taught us that common sense is a poor guide in new realms of knowledge. No one could have predicted this outcome, but no one should be surprised by it. Everyday experience shapes your opinions about matter and motion and space and time. Common sense says that solid matter is solid, that all accurate watches keep the same time, that the mass of material after a collision is the same as it was before, and that nature is predictable: sufficiently accurate input information yields reliable prediction of outcomes. But when science moves outside the range of ordinary experience [when it moves outside of DIV2] —into the subatomic world, for instance — things prove very different. Solid matter is mostly empty space; time is relative; mass is gained or lost in a collision; and no matter how complete the input information, the outcome is uncertain. ${ }^{98}$

\subsubsection{No causation in DIV1}

Physicists are very familiar with the way that DIV1 characteristics are the inverse of DIV2 characteristics, but it is not as widely acknowledged by philosophers. In DIV1,

95 Watson $(2004,8)$. Watson's example- "large enough to be held in the palm of the hand"-is more astute than it may first appear. Notice he does not say that what fits into the palm of the hand is visible or not. According to DIV2 logic, what can be held in the palm of your hand really is anything larger than some smaller molecules.

96 Omnès $(1999 \mathrm{a}, 3)$.

97 Greene $(2004,177)$.

98 Ford $(2004,4-5)$. Stenger $(2000,44)$ also gives a lucid passage about this issue, where he maintains that the DIV1 - DIV2 cutoff is around the level of the scientists' atoms (which are composed of nuclei and electrons) as opposed to the quantum abstract atoms. 
causation does not exist. In criticism of the invention of probabilities for describing quantum phenomena, Omnés writes:

Probabilities had appeared previously in the "old quantum theory" with the quantum jumps of an electron going from an atomic trajectory to another one. Einstein had also introduced probabilities in the emission of black-body radiation. In both cases however, it had been thought that probabilities were needed for the same reasons as in classical physics, simply because all of the laws were not yet known. Now, however, with a full-fledged theory of quantum mechanics, absolute randomness was plainly there. ${ }^{99}$

And Herbert writes:

The essence of quantum randomness is simply this: identical physical situations give rise to different outcomes. Once you get down to the quantum randomness level, no further explanation is possible. You can't get any deeper because physics stops here. ${ }^{100}$

Some philosophers maintain that DIV1 involves so-called "probabilistic causation" at the quantum level, and it seems that this cannot be denied, given the statistical nature of quantum particle behavior. But this probabilistic "causation" does not change the fact that there is pure unpredictability of the location of, for example, an electron in its "cloud-zone," as Herbert's passage discusses. I find it more appropriate to call this non-causation rather than probabilistic causation, since "probabilistic causation" seems to imply that there is some predictability in the electron's region, when in fact there is none whatsoever-it may be more likely that we will find the particle here rather than there, but ultimately it is not an issue that can be predicted. How can pure unpredictability involve the rhythm of causation in any way? Since it seems it cannot, it seems that the phrase "non-causation" is much more appropriate when discussing particle measurement than probabilistic causation. This can be further clarified by a passage from Matthieu and the physics professor Trinh Xuan Thuan from a passage in their book on Buddhism and physics:

Up through the nineteenth century, classical science argued that objects had an intrinsic existence governed by well-determined laws of cause and effect. But quantum mechanics, which was developed at the beginning of the twentieth century, seriously undermined the idea that the basic ingredients of matter have such a definite existence, and also raised doubts about whether the world was governed by strict rules of cause and effect. The Buddhist idea of emptiness [the insignificant duration of quantum abstract atoms] seems to be in harmony with the quantum view of reality. ${ }^{101}$

\subsubsection{DIV1 cannot compose DIV2}

DIV2 involves objects that have structure and shape because they have spatial extendedness and a surface and color, but in DIV1 this is not the case, and objects involve none of these. DIV2 involves space and time, but in DIV1 space and time do

\footnotetext{
99 Omnés (1999a, 43).

100 Herbert $(1985,118)$.

101 Matthieu and Thuan $(2001,13)$.
} 
not exist. ${ }^{102}$ In DIV2 a human witnesses what appear to be composite items that have surface and color are observed (e.g., due to its surface and colors, a chromosome molecule is observed in the microscope, and a galaxy through a telescope can be observed due to its surface and color), but in DIV1 composite items are not found.

There seem to be paradoxes that can be found if one asks how DIV1 can make up DIV2, since that would mean that a spaceless, timeless, unextended, non-causal, structureless, colorless reality that only contains point-objects makes up a spatial, temporal, causal, structured, colorful reality that contains extended objects. Overall, physicists and philosophers make the assumption that quantum abstract atoms make up DIV2 reality; but how this occurs is virtually unknown, and discussion of this is virtually ignored. ${ }^{103}$ What is important for the purposes of this article is that if DIV2 contains mereological composites, and DIV1 does not, then DIV1 items are real, and DIV2 items are conceptual-mental items (they are unreal items). In DIV2 experiencing, the experiencer has no idea that what she/he is experiencing is an illusion, and that the language she is using does not denote anything but her/his own mindbound concepts. The reasoning of this article shows that there is no DIV2 world that quantum abstract atoms give rise to in the first place, and thus it will remain one of the primary unsolved mysteries of science until it is acknowledged that it is an unsolved mystery because there cannot be any composing of the empirical world out of quantum abstract atoms.

\subsubsection{How can a structureless quantum atom be detected by a computer that does not exist? The I-empirical apprehended through the empirical}

Above I have more than once referred to the process used by scientists to detect quantum atoms I-empirically by using quantum instrumentation. What is involved in this process is the following: from the non-existent (conceptual) DIV2 dream-realm, scientists discover the real entities (quantum atoms) of DIV1. This involves using material machines and computers to detect immaterial unstructured particles. According to mereological nihilism, those material machines and computers do not exist: they are products of conceptualizers (minds that generate an empirical reality). In other words, quantum abstract atoms are detected via items that do not exist outside of consciousness. Is this is a conundrum for mereological nihilism? I will explore this issue in this subsection.

\footnotetext{
102 At the most fundamental quantum domain of reality that physicists discuss, and which they call the Planck scale, these physicists, scientists inform us that reality is "timeless" and "spaceless," in the words of Brian Greene, a leading string theorist (Greene 1999, 379).

103 A good example of the avoidance of this discussion is found in Kane (2000), chapter 2 (especially around pp. 19-22), where Kane mentions how quantum atoms (he even uses this word, "atom") "build up rocks, cells, and all of the world around us." (20) But no description of just how this occurs is found-no description of how the quantum atoms can compose material surfaces and colors is given. Kane's example here is just one of seemingly countless examples of how this issue is avoided. My guess is that the discussion is ubiquitously avoided since there are no theories that specifically describe how this occurs. The mereological nihilist, of course might say that there are no theories since it is an absurdity to believe that quantum atoms can build up a reality of composite or empirical items. Grünbaum (1952) attempted to describe how extensions composed of points-which would be a huge step forward in solving this problem-but the alleged solution depends entirely on invisible topological metaphysical relations. I attack his theory in Sect. 4.
}

Springer 
Partless quantum objects are known through indirect empirical means. Quantum atoms are experienced through the DIV2-experineces of dots on computer screens or wall screens that are generated by quantum instrumentation through spectroscopy and other quantum detection processes. In other words, scientists have empirical (DIV2) experiences of images of quantum atoms, and these empirical experiences are therefore illusions of I-empirical items. Dots, splotches and splats on various sorts of screens are all DIV2 objects, and thus all dot experiences are DIV2 ordinary empirical objects. But if, according to mereological nihilism, all DIV2 vision experiences are illusions (fabrications of conceptualizers), how do we know that these dot experiences are not also to be rejected, just as the mereological nihilist rejects any other DIV2 object? In other words, real DIV1 objects are invisible, but can be known through visible (DIV2-experiences and conscious events) by nonexistent DIV2-empirical dots and data. Is there a problem with the idea that the computer which conveys this information does not exist, but the quantum atom which is discovered with the non-existent computer does?

I will next with show that this is not a problematical issue for quantum mereological nihilism. If one merely inquires into the properties of the surfaces and colors that are given in empirical experience, one finds that the surfaces and colors are absurd, and cannot exist. It is like looking very carefully into the details of a mirage: looking carefully only reveals that the mirage does not exist. I will describe how the very act of carrying out critical analysis of any DIV2 surface and color experiences leads to a disintegration of the surfaces and colors into structureless quantum atoms. In other words, the empirical-material DIV2 perspective collapses on itself due to its inherent wrongness, and the product of that collapse, what comes out of that collapse, is quantum atoms.

Consider a wood table. From the perspective of ordinary empirical-material consciousness, I can cut it into halves. I can cut the halves into quarters, the quarters into eights, and so on until the items I am cutting are no longer empirical with my unaided eyes. The items of nature do not slice in the way that mathematical objects do. For example, in my high school geometry class, I could dissect a sphere any way I wanted. There were few, if any, restrictions. This is not how the items of DIV2 empirical-material nature dissect. If I cut apart a table, much of my cutting will consist of merely separating wood fibers and/or the plant cells that the wood consists of in DIV2 reality. This is different than the sphere in my geometry class, which could slice in any way I chose, and not in a way that was determined by the structure of the tiny constituents of the geometrical shape, as is the case with the pieces of the table. Further, if I wanted to cut in half the wood fibers or plant cells, that process would largely consist of separating the molecules that compose the fibers and cells. When I get down the scientists' atom, I make a split from an item which is perceived in DIV2 reality as having parts and an extension (such as the diameter of a scientists' atom via spectroscopy). I am quite limited in how I can split the scientist's atom, since there are only a few items to choose from in order to do with splitting, unlike the geometric object. Starting from the wood table, I have dissected down to the scientists' atom, but splitting the scientists' atom is a different sort of splitting than the previous processes of splitting, such as from table to table-halves, or plant cells to molecules. Splitting the scientists' atom is the last split, since that splitting process leads to items that are partless and not able to be further split, namely quarks and electrons. As mentioned, I am going to show in a section below that items that do not have parts can only be point-sized. For that reason, splitting the scientists' atom can 
only be the splitting from DIV2 to DIV1: from items with surface and color, to items that are unstructured and surfaceless. The quantum physicist will empirically witness DIV2 splotches and dots on wall-screens and computer screens representing these partless DIV1 items, but those representations are inaccurate since they are DIV2 extended items (e.g., a tiny patch of light appearing to be a millimeter across on the computer monitor) that represent DIV1 unextended items. Since partless items can only be points, the quantum scientist will know that the splotches and dots on wall-screens and computer screens are not completely accurate: they represent quantum atoms, but the representations have to be interpreted as approximations, since they are meant to represent points, but do not appear as points when they appear as splotches and dots on wall-screens and computer screens. For reasons just given, ordinary empirical-material experience can only reveal the existence of quantum point-atoms. In other words, if one merely inquires into the constitution of the items of their ordinary empirical-material experience, they can only arrive at the mereological nihilist thesis: the surfaces and colors of empirical-material reality can only be shown to be in diametric opposition to its constituents, wherein one must further ascertain which domain of reality is real, the surfaces and colors, or the pointatoms. This article shows that the latter are real, and the former the fabrications of conceptualizers.

\subsubsection{DIV1 and DIV2 are the inverse of one another}

The following table shows a summary of some of the details involved with the polarity between DIV1 and DIV2:

\begin{tabular}{ll}
\hline DIV1 & DIV2 \\
\hline Real & Imaginary and conceptual \\
Invisible (not available to ordinary empirical experience) & Empirical \\
Non-causal & Causal \\
Does not involve surfaces and colors & Involves only surfaces and colors \\
Objects are sizeless & Objects have size \\
Immaterial & Material \\
Quantum objects & Non-quantum objects \\
Do not persist & Persist \\
Coherent & Paradox and antinomy \\
Simple & Complex \\
\hline
\end{tabular}

It is important to remember that many of the ideas in this article are not necessarily new, and other than the arguments against composite objects in this article (in Sects. 3 and 4 and in some of the footnotes), many of the ideas presented are not original. It is only that this sort of philosophy is oddly out of favor in contemporary philosophy, and it is supplanted by the realist-empirical (ordinary empirical) theories, which paradoxically can only be given in terms of non-empirical and antinomous metaphysics. To illustrate the long tradition of the sort of philosophy being articulated in this article, consider a passage from Kant, where he is writing about Leibniz, and which illustrates Kantian transcendental idealism, which is, as I understand it, very closely aligned to the type-II dualist mereological nihilism (Sect. 2.3 above) of this article:

...space is in no way a property that attaches in itself to any thing at all outside our senses. It is, rather, only the subjective form of our sensibility, 
under which objects of the outer senses, with whose constitution in itself we are not acquainted, appear to us, and we then call this appearance matter... The metaphysician was then free to composed space out of points, and matter out of simple parts, and thus (in his opinion) to bring clarity into this concept. The ground for this aberrations lies in a poorly understood monadology, [a theory] which has nothing at all to do with the explanation of natural appearances, but is rather an intrinsically correct platonic concept of the world devised by Leibniz, insofar as it is considered, not at all as objects of the senses, but as thing in itself, and is merely an object of the understanding, which, however, does indeed underlie the appearances of the senses. Now the composite of things in themselves must certainly consist of the simple, for the parts must here be given prior to all composition. But the composite in the appearance does not consist of the simple, because in the appearance, which can never be given otherwise than as composed (extended), the parts can only be given through division, and thus not prior to the composite, but only in it. Therefore, Leibniz's idea,... so far as I comprehend it, was not to explicate space through the order of simple beings next to one another, but was rather to set this order alongside space as corresponding to it, but as belonging to a merely intelligible world (unknown to us [i.e., it is not empirical and does not have a surface and/or color]). Thus, he asserts nothing but what has been shown elsewhere: namely, that space, together with the matter of which it is the form, does not contain the world of things in themselves, but only their appearances, and is itself only the form of our outer sensible intuition. ${ }^{104}$

Although amid the modern philosophical environment it is not common to find anti-realist theorization of this sort, through the history of philosophy this trend has been very prevalent, often even being the dominant philosophy of entire cultures and eras. There are many examples, but a primary one I am emphasizing in this article is the pre-Classical Indian Buddhist tradition, due to the fact that it is so similar to the scientific philosophy of quantum abstract atomism. While the point of this article is to discuss quantum philosophy and the evidence for mereological nihilism, and not the commonalities mereological nihilism has with subtraditions in Buddhism, it is perhaps worth noting that I don't think I can hide the obvious fact that there are deep similarities between mereological nihilism and some aspects of the Buddhism of India. This is most apparent in the analysis of the quantum abstract atoms, conceptualism, immaterialism (discussed below), and the philosophy of DIV2 unreality that has been discussed in this subsection. Consider this passage from Feldman in a recent article on the Buddhist, Vasubandhu:

The inference from illusion appears at the beginning of the Vimśatik $\bar{a}$ here Vasubandhu states:

This is consciousness only, because there is the appearance of non-existent things, just as a person with cataracts sees non-existent hairs, moons, et cetera...

104 Kant (2004, 44-45). 
[This] is... a positive inference, designed to establish conclusively that external objects do not exist... It follows from the appearance of these seemingly external objects that they are merely appearances in the mind. ${ }^{105}$

And lastly, in considering philosophies that have similarities to mereological nihilism due to the rejection of ordinary empirical reality (and other similarities), there are few more relevant traditions than the one I am primarily concerned with in this article than, of course, modern physics. Consider the following passage from one of the world's most famous physicists, in a passage about time, who so clearly points out the level-headedness in rejecting, rather than accepting, the reality of ordinary empirical experience in favor of scientific information:

Certainly... the feeling that time flows is deeply ingrained in our experience and thoroughly pervades our thinking and language. So much so, that we have lapsed, and will continue to lapse, into habitual, colloquial descriptions that refer to flowing time. But don't confuse language with reality. Human language is far better at capturing human experience than at expressing deep physical laws. ${ }^{106}$

\subsection{All DIV2 experiences are experiences of surface and color that are constructions of consciousness}

In the last part of this subjection, I will defend why above I made the claim that all empirical conscious constructions that are (believed to be) about an external reality are experiences only of surfaces and colors. I will consider that every experience a human has about alleged "mind-independent" empirical reality is only an experience of a surface and/or a color. Other philosophers have also described external empirical experience this way, as if at least predominantly involving experiences of surfaces and colors. Strawson writes:

You look out a window, and you see an armoured personnel carrier rusting under a tree on the far side of a river. In such a case, you take in a spatially distributed array of color patches... [T] fundamentally determined by your sense of position relative to other objects, your immediate and automatic judgments of size, three-dimensional space, and distance, and your equally immediate experience-conditioning deployment of special concepts, like the concept of tree and water. ${ }^{107}$

I have not found an exception to this idea that all empirical experience constructions of the supposed external world are experiences of surface and/or color (an "array of color patches"). A piece of glass is a surface that might not have a color, depending

\footnotetext{
105 Feldman (2005, 529).

106 Greene $(2004,142)$. Greene is writing this at the end of a discussion about the illusion of timeflow in Einsteinian relativity, which mereological nihilism attacks. The point of giving this passage is merely to show how commonplace it is in the domain of physics to reject and/or seriously question ordinary empirical experience. It is interesting, however, to note how Greene does not mention that time is metaphysical (outside of experience), as I discuss in 5.2-as if he is not aware of that issue (it seems must humans are unaware that their experience of time is an illusory one). The findings in this article (and of Grupp 2005a) attack both the idea that there can be something like time as a feeling (of time-flow), and the time as a scientific item (such as with relativity theory).

107 Strawson (1994, 3-4).

型 Springer
} 
on the circumstances of the glass (how clean it is, etc.). Heat and sound are empirically experienced by humans, but they are also instances of the DIV2experience of surfaces. With respect to sound, as far as the DIV2-experinecer can know, it consists of the air-surface hitting the eardrum and which supposedly gives rise to the experience of sound. This is surface experience: ear-drum is contacting disturbed (sound-producing) air-surface in DIV2 reality. Experience of heat also is the DIV2-experience of an air surface with certain properties contacting the surface of the body.

In this article I am considering surfaces and colors as they are experienced. When a person sees a cloud, they do so because witness a white surface and color patch in the sky in their DIV2 experiencing. If it happens to be the case that they believe that the cloud does not involve a surface, even thought the whiteness and surfaceness is obviously witnessed in DIV2-experience, it is typically because the DIV2experiencer has been told it is not a solid item, such as if a science teacher told them that the cloud is composed of vapor. But regardless of what they have or have not been told, if they are going to have cloud-experience they will only experience the cloud as a surface and/or a color, such as the white surface seen when looking at the cloud in the sky. The DIV2 conscious constructions persons typically refer to as the experiences produced by the five senses are all described in terms of surfaces and colors. Smell is describable in terms of air surface hitting the inner nose surface. Hearing is describable in terms of an eardrum hitting a disturbed (i.e. noise-producing) air surface. Taste is describable in terms of the surface of food hitting the surface of the tongue. Tactile experience is describable in terms of the interplay of surfaces: skin surface to surface of a physical object. Sight experience is describable in terms of the experience of colors and surfaces (light reflecting off of a surface). A strong wind blowing on an autumn day pushes against our chest, and we say we feel the air. It appears to be a necessity of any description of DIV2 experience of external reality that such a description be given in terms of surfaces and colors. We cannot understand reality, including the interaction of DIV2 bodies of reality, without surfaces, extension, volume, and magnitude. Without these, we do not understand how things can contact.

Some philosophers have suggested to me that the color-experience is not the experience of a surface, but the experience of a wavelength of light that has reflected off of a surface. In a similar vein, some philosophers have suggested to me that heat is not the product of the air surface, but of the quantum particles of the body of air. I do not consider this option, however, since in considering DIV2 I am only concerned with reality as it is experience empirically, and external empirical reality is only experienced as a surfaces and colors. As far as I know, there are no exceptions to this rule. For example, even perfectly clear class (i.e. glass so clean that the surface is invisible to the ordinary empirical observer), which is apparently a colorless surface, will nevertheless only be experienced in DIV2 conceptual reality as a surface, such as if it is touched, tasted, if sound is altered because it bounces off the clear glass.

On a side note, I find wavelength claim made at the outset of the last paragraph very interesting. Those who have suggested these ideas to me also claimed to be antireductionists. But to maintain that the feeling of heat, seeing a color, or the hearing of a sound is due to quantum particles would appear to be a reductionist position, since the macroscopic DIV2 effects are apparently the result of quantum particles according to the wavelength claim, as if the heat properties of the air are only due to particle motion, and thus is best describable in terms of quantum particles. And to 
maintain that color is the result of the experience of photons (quantum particles of light) is also to have a macroscopic experience that is due to quantum effects. It seems that anti-reductionists cannot maintain that macroscopic items can be described in terms of quantum effects lest they be susceptible to reductionism.

Stroll claims that there are surfaceless things we frequently experience, such as shadows, lightening, and rainbows. (Stroll 1988, 32) But I am not sure I understand how this can be, as these seem to be mere DIV2 surfaces. My claim above is that we experience all of external reality as surfaces and colors, and this certainly holds for shadows, lightening, and rainbows. Shadows are experienced as color patches of some area that is not as bright as the color patches of the same material-say a piece of concrete-around it. So the shadow just appears to be the DIV2 perception of concrete (surface) under darker lighting conditions. I don't see how that is not a surface, when a fluctuation in the level of light is all that is at stake. If I have a bright room, and I make the light even brighter, just altering the level of light in my DIV2 experiencing, the ordinary empirical DIV2-experiencer will not maintain in that case that the brightness has given rise to an alteration of the surfaces. So why is a shadow a different circumstance? As for lightening, it is experienced as a white or golden line suddenly appearing in the sky, which is perceived to have some thickness, and thus is perceived as a surface due to that thickness. So lightening experience is surface experience. Rainbows also are perceived as surfaces with striped colors, and as the cloud example, we will only believe they are not surfaces if we are told they are vaporous. Stroll also maintains that things like trees and cats do not have surfaces (33-36). I think that what Stroll means is that there is not one DIV2-perceived color patch that is a surface of these items (assuming one is looking up close enough-from a distance, a crow will surely appear to have a smooth, continuous surface with no variation, but up close this is not the case, since the details of the feathers will become noticeable). This is true of almost any surface that is witnessed in DIV2 experience: from a distance DIV2-experineced items involve smooth and unbroken surfaces (the black crow from a distance), whereas if one moves closer, details will appear (such as the feathers on the crow) and what was considered a smooth unbroken surface will closer-up will be experienced to have clumps of color patches, but where at the closer view, the clumps themselves will then be viewed as continuous unbroken surfaces (example: a piece of the feather that appears to be one color). In DIV2, the closer-up to an object we move in our experiencing, the more the smooth patches will gain more detail and cease to be smooth. Smooth patches will be revealed to involve separate clumped patches which, in turn, appear smooth and continuous, until the observer moves in closer. What is perceived at one scale as smooth and unbroken will be shown to be a false view at a larger or smaller scale. Failing to recognize this relativity of DIV2perception experience gives rise to countless errors in academic work.

\section{A new argument for the impossibility of composite objects}

In this article I give several novel arguments for the non-existence of composite objects. These arguments show that it is not only the case that quantum science gives evidence for mereological nihilism, and reveals the existence of the nihilistic 
quantum abstract atoms, but philosophical reasoning does also. In this section, I will first give the simplest argument for this thesis.

Composition is typically described to be something like this:

If $\mathrm{a}$ and $\mathrm{b}$ compose whole $\mathrm{C}$, then it follows that $\mathrm{a}$ and $\mathrm{b}$ compose something, and if they do then there is an entity (C) that a and b compose.

This description makes it appear that $\mathrm{a}+\mathrm{b} \neq \mathrm{C}$ since, according to the antireductionist assumption in the description, $\mathrm{C}$ is not just its parts ( $\mathrm{a}$ and $\mathrm{b}$ ); rather, it is something more than the parts, and, ipso facto, $\mathrm{a}+\mathrm{b} \neq \mathrm{C}$. Another way to put this issue is this: a whole that is made of parts cannot be fully describable in terms of those parts lest reductionism ensue.

Consider the following diagram of object $\mathrm{C}$, which is an object that is not reducible to its parts, a, b, c, d.

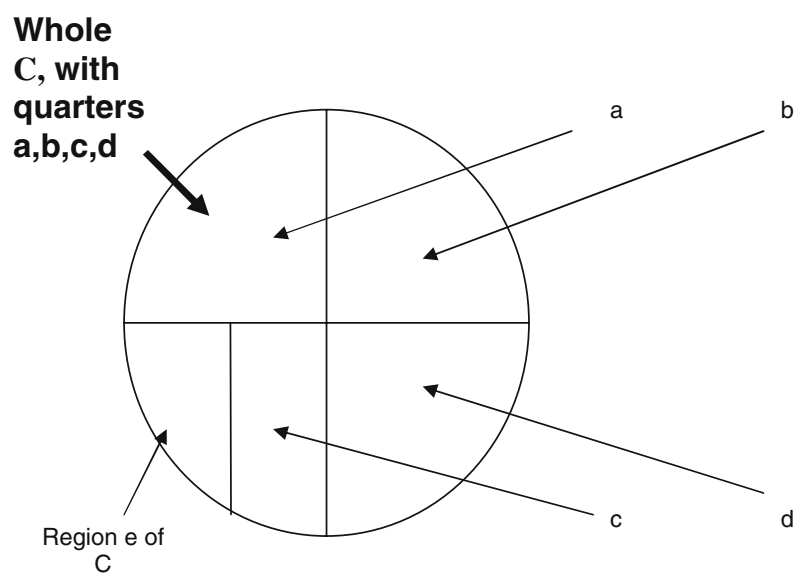

Actually, according to traditional mereological account, $\mathrm{C}$ can be considered to have five proper parts, a, b, c, d and the interrelatedness (part-whole) relation among $\mathrm{a}, \mathrm{b}, \mathrm{c}, \mathrm{d}$, and $\mathrm{C}$ (call the interconnectedness I). In order to avoid reductionism, C cannot be describable only in terms of these proper parts, since according to the antireductionist, the whole is not identical to the parts. This is a standard way to consider wholes in non-reductive metaphysics of objects, and in the philosophy of emergentism. For example, Hoffman and Rosenkrantz write: "...as we have argued, a material object in the ordinary sense cannot be identified with... a collection or mereological sum." 108 Putting the matter in different words, we can consider whole, $\mathrm{C}$, is not equal to the set of parts, [a, b, c, d, I]:

$$
\mathrm{C} \neq[\mathrm{a}, \mathrm{b}, \mathrm{c}, \mathrm{d}, \mathrm{I}] \text {. }
$$

Considering the whole as distinct from the parts is a standard position, as Wiggins discusses, in a passage where rather than using the symbols " $C$ " and "a", "b", "c", "d", and "I", he uses " $T$ " and " $W$ ":

$\overline{108 \text { Hoffman and Rosenkrantz }}$ (1997, 79-80). 
A certain tree $T$ stands (leafless, suppose) at a certain spot at time $t_{1}$ and occupies a certain volume $v_{1}$ at this time $t_{1}$. All and only $v_{1}$ is also occupied by the aggregate $W$ of the cellulose molecules which compose the tree. Indeed it is their occupation of $v_{1}$ which precisely determines that the volume which the tree occupies is volume $v_{1}$. The tree $T$ and the cellulose molecules $W$ are thus in exactly the same place at exactly the same time. Are they identical? Now $T=W$ only if whatever is true of $T$ is true of $W$ (Leibniz' Law). It follows, I think, that $T=W$ only if $T$ and $W$ have exactly the same conditions of persistence and survival through change. But self-evidently they do not.

Suppose $T$ is chopped down and then dismembered and cut up in such a way that no cellulose molecule is damaged. It seems that $\mathrm{W}$ then survives. And there is just as much wood in the world as there was before. But $T$, the tree, cannot survive such treatment. Conversely, suppose the tree is pruned and the clippings are burned, or that it undergoes an organic change which destroys some of the original wood cells. Then the tree $T$ survives but $W$, the aggregate defined as the aggregate of such and such particular cellulose molecules, does not survive. ${ }^{109}$

We can consider $\mathrm{C}$ in ways that are different than the set [a, b, c, d, I]. (Below I will refer to this set of C's parts as $\mathrm{P}$, so that $\mathrm{C} \neq \mathrm{P}=[\mathrm{a}, \mathrm{b}, \mathrm{c}, \mathrm{d}, \mathrm{I}]$ ) If the whole (C) cannot be reduced to parts, and cannot be describable in terms of parts, then the whole is, in some sense, partless. The crux of my argument in this section, and which I will give below, has to do with this issue of the apparent partlessness of $\mathrm{C}$, which I will focus on next.

If $\mathrm{C}$ is not only its parts (i.e., if reductionism is wrong), as just discussed, then in some sense $\mathrm{C}$ is not composite (it is partless). In other words, a whole is a partless item that is distinct from the parts.

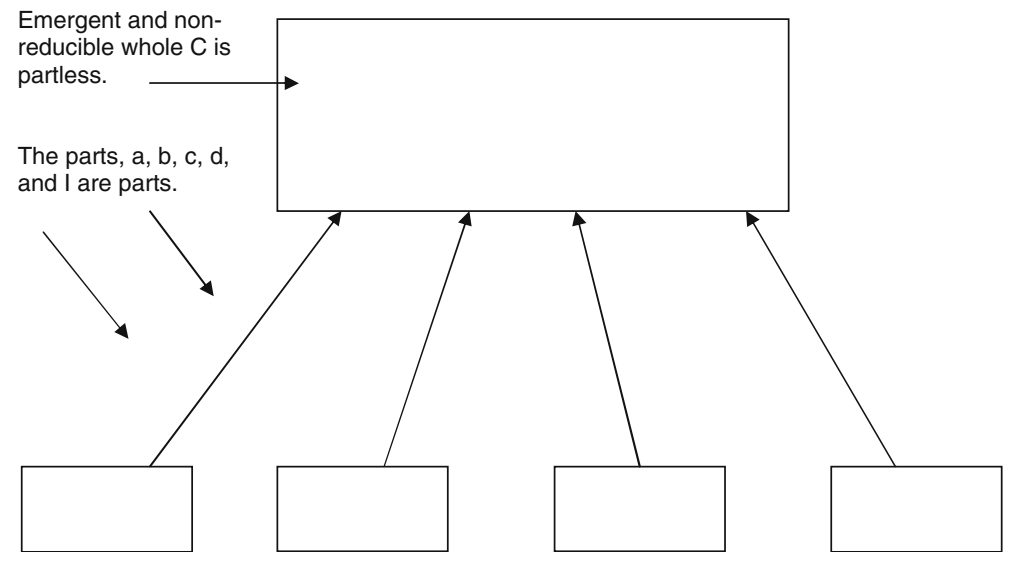

I imagine that some philosophers will object to the idea that $\mathrm{C}$ is partless item, since they may assert that $\mathrm{C}$ cannot be a true partless item, since, to the nonreductionist, $\mathrm{C}$ is intended to be a whole that is more than its parts. It is whatever is meant by "more than" that I am referring to when I maintain that $\mathrm{C}$ is a partless item. If $\mathrm{C}>[\mathrm{a}, \mathrm{b}, \mathrm{c}, \mathrm{d}, \mathrm{I}]$, then I do not see how I can avoid the conclusion that there

\footnotetext{
109 Wiggins (1997, 4).

Springer
} 
is something, call it $\mathrm{C}_{\mathrm{A}}$, about $\mathrm{C}$ that does not involve [a, b, c, d, I]. And I do not see a way to avoid the conclusion that whatever it is that is "more than" $\left(\mathrm{C}_{\mathrm{A}}\right)$ is truly partless. I will come back to this idea that $\mathrm{C}_{\mathrm{A}}$ is partless in a few paragraphs, and when I do I will find that $\mathrm{C}_{\mathrm{A}}$ is an impossible item, wherein objects made of parts are impossible.

Reductionism involves the idea that composite object $C$ is only its parts; call this principle $S_{R}$. The only way to avoid $S_{R}$ is to merely maintain $\sim S_{R}$, the negation of the statement " $\mathrm{C}$ is only its parts", which is $\sim(C$ is only its parts $)$. This statement can be written it is not the case that $C$ is only its parts, which we are calling $\sim S_{R} \cdot\left(\sim S_{R}\right.$ leads to the conclusion that there must be partless item $C_{A}$.) It appears that $S_{R}$ and $\sim S_{R}$ exhaust all the possibilities that there can be for $\mathrm{C}$. But I have noticed that many philosophers tell us that $\mathrm{S}_{\mathrm{R}}$ and $\sim \mathrm{S}_{\mathrm{R}}$ somehow do not exhaust all the possibilities that there can be for $C$, as if there is a middle between $S_{R}$ and $\sim S_{R}$. Instead, below we will see an example of how they tell us that

A. a whole is not completely distinct from is parts, and

B. a whole is in some sense distinct from its parts. ${ }^{110}$

To see an example of this, consider a passage that is a continuation of the passage given above from Wiggins. This passage represents the way anti-reductionistic philosophers attempt to maintain that $\mathrm{S}_{\mathrm{R}}$ and $\mathrm{S}_{\sim \mathrm{R}}$ do not exhaust all the possibilities:

None of this is to say that $T$ is something over and above $W$. It precisely is not. The "is" of material constitution is not the "is" of identity. The tree is made of (orconstituted of orconsists of) $W$, but is not identical with $W$. And " $A$ is something over and above $B$ " denies $A$ is (wholly composed of) $B$ " or "A is merely (or mere consists of) $B$." If $A$ is something over and above $B$, then of course $A \neq B$, but the proper point of saying "over and above" is to make the further denial that $B$ fully exhausts the matter of $A$. But $W$ does fully exhaust $T$ and so $T$ is not something over and above $W{ }^{111}$

I will next show how this passage shows that Wiggins is maintaining both $\mathrm{A}$ and $\mathrm{B}$ (and I will argue in the next paragraph that to do that is a contradiction). If we look at Wiggins's words, we see in the first sentence the assertion that, "None of this is to say that $T$ [the set of parts] is something over and above $W$ [whole];" this is statement A. (In line with what I wrote above, I do not see how this "over and above" is not a reference to the aforementioned $\mathrm{C}_{\mathrm{A}}$.) Wiggins's third sentence involves the idea that set of parts "is not identical with" the whole; this is statement B. Maintaining that the whole "[not] over and above" its parts appears incompatible with the position that the parts are "not identical with" the whole. To see why Wiggins's passage involves a contradiction, we only need to put these incompatible claims together in one statement (which we can do since both Wiggins's statements A and B are descriptions of one whole):

C. A whole $W$ is "[not] over and above" its parts, and the set of $W^{\prime}$ s parts "is not identical with" the whole.

110 To hold both $\mathrm{A}$ and $\mathrm{B}$ - as we will see with examples that anti-reductionists attempt to do this-is to say that both anti-reductionism and reductionism are not correct. (A is an anti-reductionist statement, and $\mathrm{B}$ is reductionist.) This would be to maintain that $\sim \mathrm{R}$ and $\mathrm{R}$ are admitted, which leads to this: $\sim \mathrm{R} \wedge \mathrm{R}$.

111 Wiggins $(1997,4)$. 
The problem can even appear more prominent if I paraphrase statement C. It appears safe to paraphrase the first half of statement C, W "[not] over and above" its parts, to read, nothing about $W$ is found outside of its parts. And it appears safe to paraphrase the second half of statement $\mathrm{C}$, set of $W^{\prime} s$ set of parts "is not identical with" the whole, to read,there is something about $W$ found outside of its parts. If I put together these paraphrased halves of statement C, I get statement D:

D. Nothing about $W$ is found outside of its parts, and there is something about $W$ is found outside of its parts.

$\mathrm{D}$ is an obvious contradiction. The contradiction is generated by attempting to maintain that there is a middle between two statements that are negations of one another: $\mathrm{S}_{\mathrm{R}}$ and $\sim \mathrm{S}_{\mathrm{R}}$.

From Wiggins's first passage given in this section, we find that $T \neq W$. But from this second Wiggins passage just given, we find that $T$ is not over and above $W$. It is very difficult to understand how Wiggins wants us to interpret "over and above." (He does not define it for us, and other anti-reductionistic philosophers also do not define how a whole is not "over and above" its parts without reductionism following.) It seems that "over and above" is merely a way to describe, in evasive terms, that $T$ is its parts $W$, but not in a way that directly says $T=W$. Consider the statement " $W$ does fully exhaust $T$ " in the last sentence of Wiggins's second passage. How is this not to be interpreted as being identical to this statement: "to know $W$ means we know $T$ "'? I see no reason why these are not paraphrases of one another, and if they are, then " $W$ does fully exhaust $T$ " is a reductionistic position. If it is reductionistic, then " $W$ does fully exhaust $T$ " contradicts the position that $T$ is not over and above $W$, which is in the first sentence of the second passage, and Wiggins's position is impossible. ${ }^{112}$

And now for the problem surrounding the partlessness of $\mathrm{C}_{\mathrm{A}}$. As discussed above, $\mathrm{C}_{\mathrm{A}}$ is the aspect of Composite $\mathrm{C}$ that cannot have anything to do with the parts, which is the something that is "more than" the parts. For that reasons, $\mathrm{C}_{\mathrm{A}}$ can only be the wholeness of $\mathrm{C}$. This wholeness, $\mathrm{C}_{\mathrm{A}}$, must encompass all of $\mathrm{C}$, fully coinciding with all of [a, b, c, d, I], for if it did not, it would not serve as the wholeness of C's parts since there would be parts, such as $\mathrm{b}$, that are parts of $\mathrm{C}$ but which do not exist in the domain of the whole of $\mathrm{C}$. If $\mathrm{C}_{\mathrm{A}}$ is distinct from its parts, this indicates that $\mathrm{C}_{\mathrm{A}}$ coincides with its parts. But the whole does this in a way where the whole does not exactly collocate with any of its individual parts. For example, if $\mathrm{C}$ is a sphere, as in the diagram above, since part $b$ is not the same size of the whole sphere, then the whole sphere does not perfectly coincide with quarter $\mathrm{b}$. In other words, $\mathrm{C}_{\mathrm{A}}$ and $\mathrm{b}$ do not perfectly coincide. All of $\mathrm{b}$ coincides with $\mathrm{C}_{\mathrm{A}}$, but not all of $\mathrm{C}_{\mathrm{A}}$ coincides with $\mathrm{b}$. But if that is the case, then a serious problem with $\mathrm{C}_{\mathrm{A}}$ can be pointed out, which I will do next.

\footnotetext{
112 There is another way that philosophers try to explain $\mathrm{C}$ without maintaining that it is only its parts, but which seems to involve serious problems. If the parts (P) of $\mathrm{C}$ are interconnected parts (IP) and $\mathrm{P}$ ? IP whereby $\mathrm{C}=\mathrm{IP}$ and $\mathrm{C} \neq \mathrm{P}$, this may make it appear as of $\mathrm{C}$ is not reducible to its parts, where I is the difference-maker. (It seems that William Vallicella has either advocated this position in unpublished articles.) But this is of no avail, however, for the anti-reductionist, since I see no reason why the interconnection (I), as just described, should not be considered a constituent of C (a part of C).
} 
Each of $\mathrm{a}, \mathrm{b}, \mathrm{c}, \mathrm{d}$, and I entirely coincide with $\mathrm{C}_{\mathrm{A}}$; but $\mathrm{C}_{\mathrm{A}}$ does not perfectly coincide with a, b, c, or d (I will discuss why I does below). In the following analysis, I will also now begin also discussing part $\mathrm{e}$ in the above diagram (which is approximately an eighth of $\mathrm{C}$, approximately a half of part $\mathrm{c}$ ). $\mathrm{C}_{\mathrm{A}}$ can be described by the following statements:

S1: coincides with a,

S2: coincides with $b$,

S3: coincides with c,

S4: coincides with d,

S5: coincides with e,

S6: coincides with I.

In the diagram above, we saw that $\mathrm{a}, \mathrm{b}, \mathrm{c}$, and $\mathrm{d}$ are quarters of $\mathrm{C}_{\mathrm{A}}$, and $\mathrm{e}$ is an eighth of $\mathrm{C}$. Notice that, for example, $\mathrm{a}$ and $\mathrm{b}$ do not coincide, $\mathrm{c}$ and $\mathrm{d}$ do not coincide, and $\mathrm{e}$ and $\mathrm{b}$ do not either. Considering $\mathrm{e}$ and $\mathrm{b}$, e and $\mathrm{b}$ are not in each other's domains. The coincidence that each $\mathrm{e}$ and $\mathrm{b}$ have with $\mathrm{C}_{\mathrm{A}}$ are different sort of coincidence they have with $\mathrm{C}_{\mathrm{A}}$. This leads to the conclusion that $\mathrm{b}$ is not coinciding with $\mathrm{C}_{\mathrm{A}}$ where $\mathrm{e}$ is coinciding with $\mathrm{C}_{\mathrm{A}}$. From this I can maintain that

S6: $\mathrm{b}$ does not coincide with $\mathrm{C}_{\mathrm{A}}$ where e is.

Here is where the problem comes in. $\mathrm{C}_{\mathrm{A}}$ is partless and cannot have different parts coinciding in different ways with different things (such as with parts $\mathrm{b}$ and $\mathrm{e}$ ). If partless $\mathrm{C}_{\mathrm{A}}$ is discussed, then only the entirety of $\mathrm{C}_{\mathrm{A}}$ can be discussed. Thus, if S1 - S6 are statements that describe $\mathrm{C}_{\mathrm{A}}, \mathrm{C}_{\mathrm{A}}$ involves contradiction and is impossible, as I will next show, and since that means that all of $\mathrm{C}_{\mathrm{A}}$ must be at any of the parts of $\mathrm{C}$, and at the entirety of $\mathrm{C}$. If all of $\mathrm{C}_{\mathrm{A}}$ must be at e, for example, as $\mathrm{S} 6$ indicates, then $\mathrm{S} 7$ follows:

S7: $\mathrm{C}_{\mathrm{A}}$ does not coincide with $\mathrm{b}$.

$\mathrm{C}_{\mathrm{A}}$ is partless, and thus any statement about it can only describe the entirety of $\mathrm{C}_{\mathrm{A}}$, since there is no part of $\mathrm{C}_{\mathrm{A}}$ that can be discussed. Each of $\mathrm{S} 1-\mathrm{S} 7$ describe the entirety of $\mathrm{C}_{\mathrm{A}}$. But notice that, for example, $\mathrm{S} 7$ contradicts $\mathrm{S} 2$ : putting $\mathrm{S} 7$ together with S2 into a compound statement (we can do this since both S2 and S7 describe all of $\mathrm{C}_{\mathrm{A}}$, and we can combine them in one redundant statement that describes $\mathrm{C}_{\mathrm{A}}$ ), we get the following statement that describes $\mathrm{C}_{\mathrm{A}}$ :

S8: coincides with $\mathrm{b}$ and does not coincide with $\mathrm{b}$.

$\mathrm{S} 8$ shows that $\mathrm{C}$ involves contradiction and is thus impossible. ${ }^{113}$

\section{The impossibility of relations or contact between any entities}

In the previous subsection I showed that composite objects do not exist. In this section, I will however give much more evidence for this position by specifically attacking the ways philosophers try to describe composite objects. Mereological

\footnotetext{
113 I imagine that mereotopologists will maintain that the mereotopological idea that entities are seamless coincidences of boundaries can get around the problems just pointed out. Mereotopology requires that the boundaries contact ("contact" is the actual word used by mereotopologists), but the reasoning I present in Sect. 4 shows that there cannot be any such contacting between coinciding boundaries.
} 
ideologies are so firmly established in human DIV2 consciousness that I believe it is worthwhile to explore the specific ways philosophers, mathematicians, and some physicists attempt to describe objects as being composite in order to attack those positions in the interest of mereological nihilism. The metaphysical ways that philosophers, mathematicians and some physicists go about describing structured reality enables and promotes a mereological model of reality, and enables theorists to ignore quantum mereological nihilism. But, as we will see, their accounts are entirely metaphysical. For example, consider this passage from Hoffman and Rosenkrantz:

Our analysis of the... relation that unites the parts of a mereological compound seems to answer the monadistic [i.e., nihilistic]... arguments [against the existence of mereological wholes]..., since our analysis of the ... relation appears to have found the appropriate middle ground between the overly restrictive monadistic account [nihilistic account]. (1997, 90)

I discuss in detail at various places in this article that relating is not empirical in DIV2, and I show in this section that relating is impossible between any objects. Contacting is empirical in DIV2 experiencing, but quantum physicists widely agree that it is an illusion, ${ }^{114}$ and I will show through several arguments in this section that it is impossible. Showing that these accounts are impossible leads to the position that composite objects do not exist. I will also show that action-at-a-distance (AD) might be believed to be a way to avoid these problems I point out regarding contacting and relating, but in Sect. 4.4 I given a novel argument for what appears to be fatal problems for AD. But before discussing problems with contacting and interrelating of quantum abstract atoms, I will first discuss in detail the way that philosophers, mathematicians, and some physicists assume that there are composite items by assuming that parts touch or fasten or link to one another to give rise to wholes, or by assuming that there are relations or connections between parts to give rise to wholes.

\subsection{How are wholes (allegedly) constructed?}

When mathematicians, philosophers, and scientists discuss what they believe are the mereological wholes of nature, such as a lion that is a collection of cells, a proton that is a collection of quarks and gluons, a flock of birds, a galaxy, a beach, a portion of empty space, a collection of books on a bookshelf, or a force or field (such as the gravitational force or an electric field), they usually unconcernedly refer to these as single things (composite items, mereological wholes), even though they are made up of many other things (parts). Even when entities are considered that have distinct parts that are obviously unattached (not directly touching), as in the case of galaxies, flocks of birds and any particles that can be indirectly detected, philosophers and physicists (and just about anybody else) nevertheless typically discuss such objects as if they are single objects (wholes). The idea involved in mereological nihilism is that if it can be shown that there are no interconnections between things, just as we can easily see that there are no connections between the birds of a flock, and if there is

114 I have given many textual examples of this, but perhaps another is worthwhile. See Ford $(2004,4)$. 
no touching between items, then there is no way that composite objects can accumulate in any universe, and there is no way any universe can exist.

Those who describe material constitution and/or mereology unconcernedly assume that the idea that an item made up of other items (a whole made up of parts) is coherent, since a whole can exist due to

1. The touching or contact between the parts of the composite material object in question can lead them to accumulate into a whole, ${ }^{115}$ and/or

2. The relationships or interconnections among the parts of the composite material object in question can lead them to interlink so as to give rise to a whole. ${ }^{116}$

The following diagram illustrates each of 1 and 2, where position 2 is on the left and 1 on the right:
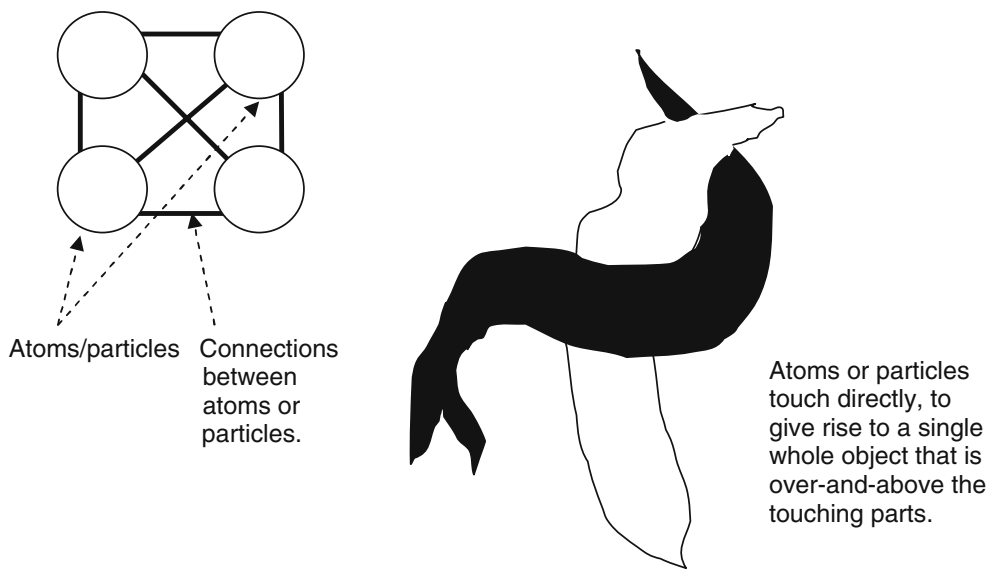

Of course touching or contacting (point 1) could just be a type of relating (point 2 above), but it seems that philosophers and physicists often discuss these separately in their research, and only very rarely make a specific point to tell us that touching is a type of relatedness. ${ }^{117}$ For that reason, I too will discuss relating and touching separately as I have in points 1 and 2 . Also, while 1 may merely be a specific variety of 2 , philosophers have often argued in a way where 2 need not be a specific variety of 1 , since philosophers typically have no problem considering that things that are not touching can be related (as seen, for example, in the Hoffman and Rosenkrantz

115 The concept of direct touching or contacting, such as when two physical items (are believed to) touch one another, is a very dubitable position, and it is not endorsed by many physicists or philosophers of physics. Lange writes:

...action by contact is not straightforward, I have just surveyed three ways of understanding two colliding billiard balls as sharing a point, as separated by a point, and as momentarily forming a continuous body. Each view encountered serious difficulties-perhaps not severe enough to prove it false, but enough to be worrisome. (Lange 2002,10)

116 For a nice discussion of this position (particles exist in relations to one another and thus give rise to objects, such as the objects humans perceive in their daily life), see Merricks (2001), chapter 1.

117 Hudson (2001a) specifically points out that touching is a relation (p. 120). Also, Hoffman and Rosenkrantz (1997, 84-86) appear to also point this out. 
passage just given, where they maintain that although subatomic particles do not touch one another, they still can account for mereological wholes), such as, for example, if two points in the manifold of space are at a distance from one another but (are alleged to) share a relation (such as the topological relation connectedness). Consider the following passage from Kline and Matheson that advocates force-interaction without touching:

[The premise,] (1) A collision between two bodies involves their touching... is highly dubitable. It is easy to imagine a mechanism for collisions whereby they occur as the result of some short or long-ranged force. Thus two objects could repel each other violently without touching, in the way that electrons repel each other. While this situation makes perfect sense, it is not a mechanistic one. According to collision mechanics, collision requires touching... To deny (1) is to subscribe to some sort of field theory. ${ }^{118}$

They are asserting that objects can interconnect without touching: interconnecting and/or relating does not have to be a way that objects touch or connect. There are a few problems with this assumption, however:

a. Don't forces have to contact and/or touch the particles they interconnect? Kline and Matheson provide no account for this particle-to-force interaction as being something other than touching or contracting. (Referring to force-connections as being the means of interaction between particles, Kline and Matheson tells us "it is easy to imagine [this] mechanism" but until we know how a field interacts with a particle, it seems almost impossibly difficult to imagine two particles interacting via a field connection.)

b. We will see in Sect. 5 below that forces have been discovered by scientists not to be metaphysical items (such as metaphysical relations between particles, as Kline and Matheson seem to believe is the case) but rather to be streams of particles. So discussing particle interaction via forces or fields is ultimately particle activity only.

There is no scientific evidence that DIV1 involves either 1 or 2 , and there are scientific reasons to believe they cannot. ${ }^{119} \mathrm{We}$ will also see philosophical reasons which show that 1 and 2 are impossible. Before going into specific argumentation that shows that 1 and 2 are impossible. I will give examples of how philosophers and physicists use 1 and 2 .

In an interesting passage I will give next, Hoffman and Rosenkrantz demonstrate both 1 and 2, showing how philosophers need 1 and/or 2 for a theory of mereological composition. In this passage Hoffman and Rosenkrantz use "monadist" to be synonymous with "mereological nihilist." And this is a particularly interesting passage since in it Hoffman and Rosenkrantz emphasize that we do not yet have a philosophy of relations, such as part-whole relations, between particles, and they discuss composition as if there is no satisfactory account of composition we have available to us, and we are still waiting for one to be given. Also, in this passage Hoffman and Rosenkrantz interestingly do not consider that this is the case because there is no account of composition, and because mereological nihilism is correct.

\footnotetext{
118 Kline and Matheson (1987, 510).

${ }^{119}$ For example, the electron, one of the most common particles that (allegedly) makes up empirical reality, cannot directly touch other particles, according to scientists. Davies (1984, 107-108). 
In the absence of a clear account of the relations which are commonly thought to unite parts of material objects, the monadist might argue that two material objects compose a third object only if they are at a zero distance from one another. The monadist [i.e., nihilist] could then appeal to the aforementioned implication of modern physics, and conclude that the common belief that there are nonfundamental material objects is mistaken. Given that there are particles (which are indivisible), it would follow that such particles are the only material objects...

As we have noted, the commonsensical view presupposes that mereological compounds and organic living things can be created or destroyed by assembly or disassembly. Thus, the skeptical attacks of the monadist and the collectivist upon the commonsense view can be answered if one can provide a satisfactory analysis of the... relations that unify or organize the parts of mereological compounds and organic living things which is consistent with this presupposition of the commonsense view. In particular, skeptical attacks of these kinds are answerable if one can provide an adequate analysis of these relations that meets [at least this] condition...... [I]t must be less strict than the monadistic [nihilistic] view that two things are united only if they are at a zero distance from one another. ${ }^{120}$

As for scientists, the standard way for chemists and physicists to consider fundamental reality, like the aforementioned philosophers, is to consider particles as being in an arrangement, where their arrangement gives rise to a composite object made up of those arranged atoms, and where if the arrangement is changed, the composite object made up of the atoms may be changed. Any college physics or chemistry textbook illustrates this, where particles and molecules are typically drawn in diagrams like these:
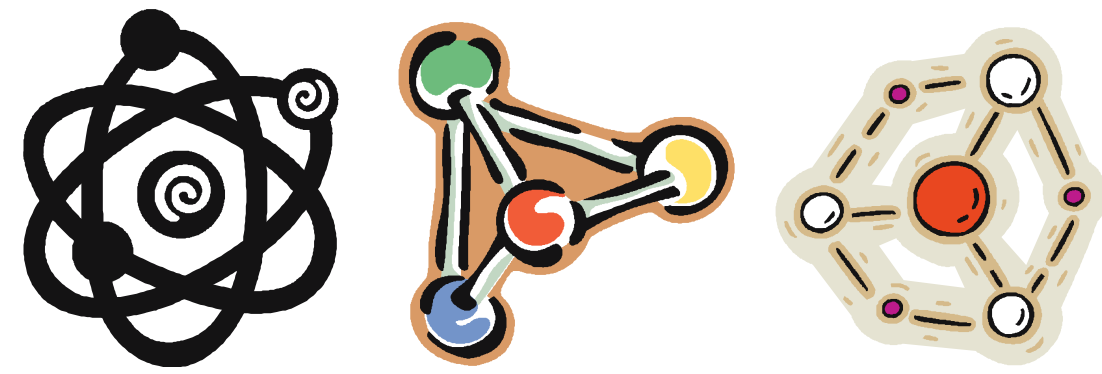

We will see in Sect. 7 below that the forces between particles and which bind particles have been discovered to be made up of unattached particles. But notice that the center and left diagrams show continuous (unbroken) connections/lines between the particles. It is these sorts of erroneous connections which foster incorrect beliefs that lead people to imagine that molecules or scientific atoms are mereological wholes. This is because these diagrams illustrate the particles as being

\footnotetext{
${ }^{120}$ Hoffman and Rosenkrantz (1997, 78-79). Markosian also writes on what appears to be 1 and 2, and as if they are the only these two options (Markosian 1998b, 212). For further discussion of 1 and 2 , see van Inwagen (1990, chapters 1-8). Some philosophers only discuss relating, apparently believing contact or touching to be a subset of relating. Consider what Horgan writes about van Inwagen on this issue and on van Inwagen's philosophy of composition from van Inwagen's book Material Beings: "[van Inwagen's] theory accommodates various plausible, deep-seated, beliefs that many or most of us have about the world-such as the belief that... whether certain objects add up to or compose some larger objects does not depend on anything besides the spatial and causal relations ... to one another." (Horgan 1993, 693)
} 
interconnected, where the connections, which are drawn as continuous (unbroken, not particle-like), directly touch the particles they interconnect. There is no evidence in any experiment of physics or chemistry that shows that the forces between particles (which is what these lines are meant to represent) are continuous-unbroken connections (in fact we will see that it is quite the opposite). Notice also that in the diagrams the particles have volume and size (and thus a surface and color), as if they are tiny balls. This is a picture of fundamental reality that is closely aligned with the billiard-ball reality of the mechanistic philosophers of the Enlightenment, and thus is a picture of fundamental reality that is hundreds of years out date, having literally nothing in common with contemporary experimental physics research. From what I can tell, contemporary philosophers are virtually unaware that forces between particles have been discovered to merely be unattached quantum abstract atoms (to illustrate this lack of knowledge, consider how Swinburne writes that: "there is nothing more to large-scale material objects except the fundamental particles and the relations they have to each other" ${ }^{\prime 21}$ ).

From what I can tell, position 2 is by far the more widely held position held by philosophers, and by the decreasing number of physicists (typically non-quantum physicists) who still happen to discuss reality or quantum reality in terms of relations. Often relations-talk, for example, will exist in quantum theoretical physics (as opposed to quantum experimental physics). Consider a passage from a recent article on quantum mechanics by Jenann Ismael, a philosopher:

The heart and soul of quantum mechanics is contained in the Hilbert spaces that represent the state-spaces of quantum mechanical systems. The internal relations among states and quantities, and everything this entails about the ways quantum mechanical systems behave, are all woven into the structure of these spaces, embodied in the relations among the mathematical objects which represent them... This means that understanding what a system is like according to quantum mechanics is inseparable from familiarity with the internal structure of those spaces. Know your way around Hilbert space, and become familiar with the dynamical laws that describe the paths that vectors travel through it, and you know everything there is to know, in the terms provided by the theory, about the systems that it describes. ${ }^{122}$

As mentioned in the introduction, mereotopology has been developed as an attempt to avoid problems with topology and mereology. But mereotopology also depends on 1 and 2 (specifically, on the touching of boundary points of extended objects, ${ }^{123}$ and the

\footnotetext{
${ }^{121}$ Swinburne $(1995,395)$. I am grateful to Trenton Merricks for this passage.

122 Ismael ((2004)), section 1.

123 Mereotopology is a theory of boundaries. Barry Smith writes:
}

We wish... to capture the commonsensical intuition to the effect that boundaries exist only as boundaries, i.e. that boundaries are dependent particulars: entities which are such that, as a matter of necessity, they do not exist independently of the entities they bound... This thesis-which stands opposed to the set-theoretic conception of boundaries as, effectively, sets of points, each one of which can exist though all around it be annihilated-has a number of possible interpretations. One general statement of the thesis would assert that the existence of any boundary is such as to imply the existence of some entity of higher dimension which it bounds. Here, though, we may content ourselves with a simpler thesis, one whose formulation does not rest on the tricky notion of dimension, to the effect that every boundary is such that we can find an entity which it bounds of which it is a part and which is such as to have interior parts. (Smith 1996, 295.) 
relation connectedness ${ }^{124}$ ), and thus if 1 and 2 are impossible as I will argue, then mereotopology is also impossible, just as mereology and topology are.

In 4.2 I will show why contacting of any sort is impossible. Then in $4.3 \mathrm{I}$ will discuss why this reveals that relations or connections of any sort are impossible.

\subsection{Fatal problems involving the alleged contacting of democritean atoms}

\subsubsection{Introduction}

In 4.2.2 I show why it is only the alleged extended philosophical atoms (philosophical atoms that have size but no parts) that are theorized to exist by some philosophers and by some physicists that can be considered to touch or contact each other. Then in 4.2.3 I will give a novel argument that shows that such extended atoms are impossible, wherein it is revealed that contacting or touching is impossible. This shows why there are no relations or connections between any items, which I show in 4.3. And in 4.4 I show why, despite what many philosophers appear to imagine to the contrary, quantum physics does not involve the idea that quantum abstract atoms touch or contact each another. I will show how the way that quantum physicists discuss their research often hides this fact. As with nearly all the issues in this article, this last point about the impossibility of touching and/or contacting will be shown to involve noteworthy agreement between the philosophical reasoning and the data of quantum physics.

Of those philosophers who allow touching and contacting between items into their philosophy of reality, they typically refer to contact and touching between particles as relations between the objects that are allegedly touching. I find it suspicious that touching and contacting are given in this case via a metaphysical description, since as I pointed out above, and as I will more below, touching or contacting is a very dubious philosophical issue, and it is usually when empirical explanations do not exist and/or when paradox and antinomy are being covered-up that a metaphysical description is invented in order give a description. Regardless, I will show in 4.3 that relations do not exist. Empiricist-oriented philosophers, and many scientists, may not engage in such "relation-talk," and may not consider touching and contact in the terminology of being "a relation." (For example, I have never read a relativity theorist assert that objects A and B are engaged in contact because they share a metaphysical n-adic relational property, touching.) In addition to attacking the metaphysical accounts of touching, in this section, I also show that non-metaphysical accounts of contacting and touching are impossible. I imagine that many philosophers and scientists will just believe contacting and touching as being as simple as a finger touching the table: one surface contacts another, and there really is not much more to it than that. I will attack that sort of undeveloped and plebian account of touching and contacting because I can prove that there are no surfaces that touch at the quantum level (Sect. 4.2.3), and if there are not, then there is no

${ }^{124}$ See Cohn and Varzi (2003, 362-365). Also, consider what Pratt and Schoop have to say about this:

Mereotopological calculi vary as to which primitives they employ, and the axioms they propose.

Clarke's calculus has a single binary relation of "connection "with the gloss that two regions are connected if they share a common point. Randall, Cui and Cohn also use a binary connection relation, but take two regions be connected if their closures share a common point. (Pratt and Schoop 1998, 622.) 
touching at all since a quantum level that involves no extension and no touching at all cannot give rise to a macroscopic level that does involve extension and touching.

In this section, I will show why there is no contact or touching between any two or more quantum abstract atoms. I will show this by showing that quantum abstract atoms can only be point-sized whereby they do not have a surface, and without a surface, there is no way they can contact or touch one another (and there is no way that quantum abstract atoms can touch any other items, such as any metaphysical items [relations, etc.] that metaphysicians might think up). It was pointed out above that it is very suspicious to imagine that quantum abstract atoms can touch or contact with each other or with any other objects. I will nevertheless imagine that there are extended quantum atoms with surfaces in order to show that, toward reductio, absurdities arise if it is postulated that atoms touch each other or touch any other objects, so as to accumulate. There are two options for the quantum atoms: they either have size or they do not. (Atoms not having size is what leads to the aforementioned philosophy of immaterialism.) Atoms that can touch or contact one another or any other items are those which (somehow) might have size (extended quantum non-abstract atoms). Atoms that cannot touch or contact one another are those that do not have size (unextended quantum abstract atoms). Next in 4.2.2 we will see why only extended atoms can contact or touch other atoms (but only in the aforementioned plebian and unphilosophical rough-and-ready way), but then in 4.2.3 I show that extended atoms are impossible, and thus there is no touching or contacting between quantum objects.

\subsubsection{Surface contacting and touching is the only sort of contact or touching}

Why is surface contact or touching the only sort of contact or touching I am considering? Here is a summary of what I will argue in this subsection. Quantum abstract atoms that do not have size, and if they are going to contact or touch each other, they must exactly coincide with one another, and if that occurred, then they would be coinciding points. But coinciding points are merely one point, and thus touching and contacting between points never occurs, since points can only unify with one another rather than keep their individuality in the act of contacting or touching. ${ }^{125}$ I will next go through these issues in detail in order to clarify why surfaceless touching or contacting is impossible.

\subsubsection{DIV2 conceptualizers of surface and color In this subsection I will expand} on the discussion from sections above where it was found that all DIV2 empiricalmaterial experiences involve surfaces and colors. In other words, if a DIV2 conceptualizer believes she witnesses something in her exteriority, it will only be a fabrication of a concept of an item that has a surface and a color. In this subsection, I will show that if an item is surfaceless, it must be a point, which is important to issues later in this article.

\footnotetext{
125 This is why, as discussed in other footnotes, the particles of physics-which are points-are observed (indirectly observed) to unify and amalgamate, rather than bump or collide with one another, when they (appear to) have any sort of interaction with one another: two points cannot bump or tap since they do not have surfaces, they can only unify and thus give rise to an entirely new particle.
}

글 Springer 
Surfaceless items can only be points, and thus contacting or touching between surfaceless items can only occur via point-sized items. The only accounts I know of where philosophers attempt to maintain that surfaceless items are extended are in two cases: vague objects like clouds or fog, and objects with scattered and/or discontinuous surfaces like trees and lion fur. In this subsection, I will be concerned with showing that this is incorrect, and each does not affect my position that the only surfaceless items are points. Such objects as those just listed, I will show, really do have DIV2 experienced surfaces (the fog is experienced as a semi-transparent body with a hazy "edge"), and/or they involve sub-surfaces (the tree may be surfaceless, but the leaves aren't), and we don't have reason to imagine that they do not.

I can explain my case for each of these by just focusing on the second account, which I will do starting in the next paragraph. But first, as for the vague objects, Sorensen (1998) elegantly argues that vague objects-objects with fuzzy, unsharp boundaries (imagine the misty "edge" of a body of fog) — do in fact only have sharp boundaries. The human DIV2 observer will only experience the vague object by witnessing what surely appears to be a color patch, and thus a surface. The misty body of fog will appear to the human observer via a color and a semi-transparent surface. So it is unclear as to why these items are really surfaceless. Furthermore, vague items like fog blobs do appear to interact with other items in the way that other DIV2 items interact with one another, such as when the fog body will reflect light, and thus one item (light) bounces off another. Also, if the fog blob extends over a highway and a truck drives through it, it will surely appear that the truck has interacted with the fog body-perhaps in much the same way a racing boat interacts with water, producing a wake, eddies and swirls through the fog. For reasons just given, I am not sure how the vague object, such as the fog blob, does not fit into being an object with a surface.

I am considering the experience of surfaces and objects with surfaces differently than the way some philosophers do, such as Stroll, ${ }^{126}$ where he holds the position that many ordinary empirical objects (trees, clouds, dogs) do not have surfaces. I will not be overly concerned with whether or not Stroll is correct on this (although some of what I have to say will lead to the conclusion that he is incorrect), since that issue is not extremely relevant in an article on mereological nihilism, which rejects all mind-independent surfaces and colors, accepting them only as conceptual DIV2 phenomenological intentionalities. What I will be concerned with is specifically showing that regardless of what Stroll says, all experience of externality in DIV2 consciousness are experiences of surface and color: there is never any DIV2 experiencing of (what is believed to be) an exterior item that is independent of being surface experience. This is because DIV2 surface experiencing does not involve experiencing of points or any extended non-surfaces.

First, Stroll's position leads me to discussion of the fact that there is a level of choosiness involved in what DIV2 human observers will consider a surface and not a surface, and accordingly a mereological whole and not a whole. Surfaces tend to be considered smooth and contiguous: if item $\mathrm{p}$ is experienced as smooth and unbroken at moment $\mathrm{m}_{1}$, if at moment $\mathrm{m}_{2}$ it is experienced as unsmooth and/ or broken, the DIV2 conceptualizer will often believe a surface has either drastically been altered, or perhaps as ceased to exist, even if only a curving or

1261988 , chapter 2. 
bending of the surface has occurred from the DIV2 perspective, or even if only mixing or manipulating of the existing matter has occurred from that perspective. For example, a plain crisp white piece of paper will often be considered to be have surfaces from the DIV2 conceptualizer's perspective. If one crumples the paper up into a paper-wad, the same human that unconcernedly considered the paper to have surfaces before the crumpling may have reservations about considering it to have surfaces after the crumpling. By manipulating the paper, what was considered a single DIV2 surface from the ordinary empirical perspective might not be considered a surface at all after the crumpling-even though all that has changed about the paper is that it has gained some DIV2-perceived bends and creases which were not perceived to exist before the crumpling, and even though the paper's surface before-and-after involves the same level of permeability and continuity (unbrokenness) from the one moment (pre-crumpling) to the next (post-crumpling).

Other items, such as two colors (say, yellow and green) of Play-dough that have been swirled together into a smooth ball will be considered to involve either two surfaces touching, or one uniform surface, depending on the level of mixing that the DIV2-experiencer witnesses. If it is mixed weakly (such as only as much as a Yin-Yang symbol involves mixing between the black and white fish of the symbol), the DIV2-observer will likely maintain that the yellow and green are distinct items that involve two surfaces touching, even if the two colors form a smooth ball from the DIV2 perspective. With only light mixing of the clumps, the DIV2-perciever will likely report that there are two contacting surfaces, but if the yellow and green globs are mixed to the point where the distinction between the yellow and the green becomes more complicated or even blurred, a person will likely report that the Play-dough clump has one surface. By manipulating the clump, two DIV2 surfaces can be considered one DIV2 surface, even though one blob of Play-dough is smooth in either light or heavy mixing. So on this case, colors of a surface could influence the DIV2 experiencer to make somewhat arbitrary choices as to if surfaces are touching (light mixing), or if there is one surface with varied colors (heavy mixing). A magazine cover with a picture and with many different colors, or a smooth rock with many different colors-just like the heavily mixed Play-dough clump-will likely be considered one surface by a DIV2 human experiencer. But a magazine cover with one color also will be considered one surface. So the cases where a surface is considered to exist or not exist, or are considered to be one or more than one, are somewhat dependent on the mere choices DIV2-humans make.

These points show how many of the surfaces that a DIV2 conceptualizer perceives, and how one organizes their external experiences of surfaces and colors, can vary and can be rather relative. Stroll is concerned with which items we should call surfaces and which we should not, or which items have two or more surfaces and which items do not. Due to the arbitrariness of such a task as that, it seems a somewhat hopeless project, and I will show below that Stroll's account can only lead to confusion. It is a much safer project to merely accept the arbitrariness of the DIV2 conceptualizer's surface constructing in her ordinary empirical life. This surface constructing is the only thing that any DIV2 perceiver ever constructs about her external reality, for reasons I discuss at a number of places in this article (such as above in Sect. 2.5 when I discussed how heat and sound are only ways in which a DIV2 perceiver generates experiences of surfaces 
in her empirical-material states of consciousness). The idea is that if humans are going to perceive anything in what they believe to be the "external world" in their DIV2 experiencing, it will only be in the form of color(s) and/or surface(s), and whether or not it is considered to be a mereological whole, or distinct mereological wholes, is an issue for the seemingly somewhat random DIV2 consciousness construction, and not of real perception or philosophical analysis.

Stroll $^{127}$ considers some DIV2 visible items, such as trees and clouds, to not have surfaces (this only works if DIV2 perception of a tree is quite close to the tree, such as within 100 feet of the tree, as we will see). The leaves are perceived to have surfaces and thus the tree is a smattering of perceived leaf-surfaces. So if Stroll is correct, and a DIV2 perception of a tree close enough to where it does not appear at all to be one smooth surface, the DIV2 experiencer will nevertheless still only have empiricalmaterial surface experiences of the leaves. Trees are not surfaceless in the sense that they are invisible to the DIV2 consciousness; rather, it is merely the parts of the tree apprehended in DIV2 consciousness that one experiences as surfaces and colors - when, that is, one is close enough to the tree to have these sorts of experiences.

There are in fact a number of questionable claims Stroll makes in his discussion about clouds and trees not having surfaces. Consider this passage:

Surface-talk is not generally applied to certain three-dimensional entities, including some of the epistemologies of the first half of the twentieth century would have called "physical objects," or "material things." Among these are clouds, ... dogs, some planets, trees, and persons...

Clouds are constituted by layers of gas and moisture and have a top layer. But a cloud, like haze, doesn't have a surface. Unlike a submarine, which can come to the surface of a body of water, an airplane does not rise to, or even above, the surface of a cloud. We see the tops of the clouds but not their surfaces. One explanation for the withholding of surface-talk here is that the objects with surfaces must have a certain density or compactness before such talk is applicable; clouds are like most gases (argon, fog, helium) in being insufficiently dense for surface-talk to apply. Clouds lack a feature that solid objects, such as marbles, possess. In general, with respect to these latter, when we come closer to them, so that we see them better, we are able to discriminate some of their surface features more easily... With clouds and other wispy entitles, we cannot do this, and this may be another reason why we withhold surface-talk from such entities. ${ }^{128}$

Van Inwagen contradicts Stroll's claim that surface talk is not applicable to the aforementioned DIV2 objects ("clouds, dogs, some plants, trees, and persons"): "Some philosophers are happy to call clouds and forests and galaxies material objects and others are not.",129 Stroll's claim that surface-talk "is not generally applied to" some of the items he mentions is questionable. Stroll is concerned with how people refer to surfaces and how they believe them to be, but if that is the case, I have difficulty believing that most people-being good DIV2 experiencers-would tell me that the sun does not have a surface. If I consult average

\footnotetext{
127 1988, 33-38.

128 Stroll (1988, 33-34).

$1291990,17$.
} 
persons (who are DIV2 denizens) as to if the big white cloud in the sky has a surface, I just can't believe even $20 \%$ of them would say "no." Even worse, if gases are not surface-creating particle systems, then, for example, the sun would have to be considered to have no surface. The same would go with planets, like Saturn, which in the quote above, Stroll tells us are not prone to surface talk. It seems that the problem with Stroll's account is that in some cases he is trying to give an account of the surfaces of many objects (Saturn, the sun, etc.) in terms of particles, wherein those objects are in fact surfaceless. But, as this article points out, doing that only will lead to the conclusion that there are no surfaces at all (which is the mereological nihilist's conclusion). If the goal is to understand how people refer to which items have surfaces, rather than which chemical mixtures can be scientifically found to have solidity, the latter will likely be outside of the average person's knowledge and outside their DIV2 conceptualizing since the average person is not a chemist or physicist. Even scientists will refer to the aforementioned objects as having surfaces: the sun-spot solar astrophysicist will probably not have much trouble carrying out entire studies on sun-spot activity without discussion of particle activity. So Stroll, at best, can only be claiming that "[s]urface talk is not generally applied to certain three-dimensional entities, including... clouds,... dogs,... trees..."can only be the discussion that some philosophers or scientists are having. (But if that is the case, as will be shown in the next paragraph, things get only worse for Stroll, since referring to macro-properties [surfacelessness] due to particle activities only can show that all macroentities are surfaceless).

Actual surface talk among the usage of scientists and ordinary people has to do with the perceived gaps and/or bends in the DIV2 object perceived. For example, few DIV2 experiencers will have a problem maintaining that the ceramic plate or the smooth ocean-both viewed relatively up-close-have a single surface, since there are few if any perceived bends or gaps that are not extreme enough (as was the case with the crumpled paper) where they evoke the DIV2 perceiver to not refer to the plate or ocean as having surface. But if the plate is shattered, the gaps created in the plate will lead people to maintain that the plate's surface has been destroyed; or if a storm emerges on the ocean and large choppy waves emerge, many DIV2 experiencers will want to maintain from the same close-up perspective that the jerky ocean is no longer a single surface, or a surface at all, due to the countless bends and kinks in it (this is like the paper crumpling example). Stroll maintains that a cloud is gaseous, and I take him to mean that it does not bounce off of other objects in the way that liquids and solids do. (As with the discussion of heat in a previous subsection, this explanation of a cloud is a reductionistic explanation: the DIV2 macroscopic properties of clouds are described in terms of molecular or atomic properties. And it is interesting that this reductionistic position is the same position I argue for in this paper: objects do not have surfaces because they are composed of particles that do not touch or attach. Regarding Stroll's account, the question is: Why does Stroll choose to apply this reasoning only to the microscopic-level nature of a cloud, but not to, for example, the submarine's metal?) All Stroll does is discuss differences in density - which can only mean particle density-in the case of gas versus solid or liquid as accounting for an object's having or not having macroscopically perceivable surface. Stroll takes such surfaces to be explained in terms of the penetrability of the gas or the liquid and/or the manners that the gas 
can bump against a liquid or solid item (the plane can't float on the cloud but the submarine can float on the ocean), where apparently it is significant that gases might be considered more penetrable and less "bumpable" than solids or liquids. (Consistent with what I claimed above, in DIV2 consciousness construction, this however is just dependent on a certain point of view. The plane is penetrable with respect to a bullet, but not with respect to a bird or cloud. And the cloud is penetrable with respect to a plane, but not with respect to the light of the sun which is reflected off of the cloud.) I do not see anything here that indicates why the particle view has anything to do with the construction in consciousness of a DIV2 macroscopically perceivable surface, since particles in chemistry in the states of gas versus liquid or solid are in fact merely a product of the quantum abstract atoms: how quickly particles are found to change positions with respect to one another (i.e., how fast they are moving), and in either case, of gas versus liquid or solid, there are merely just the moving particles, period. (Note, however, that is it unknown how quantum states of matter-solid, liquid, gas, plasma-can give rise to actual surfaces and surface experience.) So why, specifically, there is the claim that one leads to a surface and the other does not, is irrelevant, and it seems a feeble attempt to do what Stroll is really intending to do: merely talk about the macro-experience of surfaces. We already saw that the case of the tree is not a reference to surfaceless experiencing. Stroll is using the idea that, for example, trees and clouds involve interstices, but in the former humans do not typically see them or think of them, and will refer to a cloud's surface. And in the case of the tree, humans will merely refer to the surfaces of the leaves since they can clearly see the interstices between the leaves. So it appears that how humans are looking at things in DIV2 conceptualist experience, and if they can see gaps between surfaces, will it be concluded that objects have surfaces or not. If the same tree-observing human steps into a plane and looks at the forest from 10,000 feet up, she will likely refer to the forest as having a surface, associated with her experience of the somewhat smooth green continuous earth's surface. So in looking at actual language usage, rather than an abstract idea, we can see that Stroll's claims fail. Based on what has been written here, I reject Stroll's account of surfaces and surfaceless visible items (trees, etc.), in favor of my simpler account: we define surfaces by (1) actual DIV2 experiencing of them, and by (2) how people refer to them in their actual language usage.

From what has been discussed in this subsection, I am not sure how there can be any surfaceless extended items in DIV2 reality. If an item is surfaceless, it is unextended: a point. The supposed surfaceless items in DIV2 reality that Stroll mentions are either scattered objects (trees) that have surfaced parts (and thus ultimately involve surfaces in DIV2 consciousness), or they are vague objects that are visible with surface and color, and thus I am not sure how those are not surfaced items. Often philosophers will maintain that items need not have surfaces to interact, such as with the magnet pulling the metal at-a-distance, or with the stars in the galaxy allegedly interacting without contacting in order to give perceived shape the galaxy. But these are clearly quantum mechanical effects involving surfaceless particles, which do not touch, contact, or relate,. And thus those quantum mechanical descriptions are not harmful to the reasoning of this article. From what I have given, it appears that there are no extended unsurfaced items in DIV2 reality, and if an item appears in DIV2 reality to the DIV2 conceptualizer, it will have a surface. 
4.2.2.2 Contacting quantum atoms can only be one atom For reasons I will explain in the few paragraphs, quantum abstract atoms that contact or touch can only lead to the amalgamation and identity of the quantum atoms. ${ }^{130}$ This would prevent there from being any sort of contacting or touching between quantum atoms. There would not be two items contacting or touching, and the quantum atoms involved would not remain individually "themselves" throughout a contact/touching interaction. Pointsized quantum atoms, call them $\mathrm{A}_{1}$ and $\mathrm{A}_{2}$, when involved in what appears to be contact or touching, in doing so, actually coincide and compose one quantum atom. (Any distance between $\mathrm{A}_{1}$ and $\mathrm{A}_{2}$ means there is a gap between them.)

In discovering that contacting or touching of partless point-sized quantum abstract atoms does not occur, this will also reveal that composite point-sized quantum abstract atoms do not exist. If $\mathrm{A}_{1}$ and $\mathrm{A}_{2}$ are partless, then touching or contact between them involves the touching or contact of the "totalities" (their "entireties") of each of $\mathrm{A}_{1}$ and $\mathrm{A}_{2}$. A partless point can only be considered an undifferentiated oneness (I will consider an objection to this in the next paragraph), for lack of a better term. Two contacting or touching undifferentiated onenesses that are continuously integrating (contacting, touching) does not reveal anything but a single undifferentiated oneness upon that continuous integration: the contacting or touching of $\mathrm{A}_{1}$ and $\mathrm{A}_{2}$ would not result in anything but undifferentiated oneness. The item that comprises their continuous integration (contacting, touching) is also an undifferentiated oneness. Twoness cannot emerge out of undifferentiated oneness, difference cannot emerge out of non-difference, and thus the merging of undifferentiated oneness $A_{1}$ with undifferentiated oneness $A_{2}$ leads to a single undifferentiated oneness $\left(A_{1}=A_{2}\right)$ since the points are identical upon merging. In other words, the touching or contacting of the entirety of two partless, unstructured, collocated, point-sized quantum abstract atoms also gives rise to a point-sized, unstructured, partless, point-size quantum abstract atom. For these reasons, quantum abstract atoms that supposed touch or contact in fact do not involve a process of touching or contacting, and instead only involve an indistinguishability. ${ }^{131}$ If that is the case, and if the quantum atoms unify and amalgamate into just one undifferentiated oneness at the moment of their supposed touching or contacting, rather than remaining distinct individuals throughout the process, then contacting and touching between point-sized quantum abstract atoms does not exist, and rather, amalgamation, unification, and identity occurs instead: there was first the appearance of two atoms, but then only one.

I imagine that some philosophers may assert that some sort of property mereology could get around this issue, since with property mereology one can maintain, for example, that point $\mathrm{A}_{1}$, although partless and unstructured (it has no pieces that are

\footnotetext{
${ }^{130}$ Of course, the quantum abstract atoms were indistinguishable before amalgamation, for reasons discussed in Sect. 1 about the indistinguishability of quantum abstract atoms. But as I also said in Sect. 1, I have no choice but to discuss quantum abstract atom as if they are not one (but rather are many), since the ordinary materially oriented human conceptualizer will be conditioned by their language and thought development in DIV2 reality to believe that non-coinciding quantum abstract atoms are not one, but are more than one. The point of what has been just brought up in this first sentence of this subsection is that "touching" and "contacting" among quantum atoms does not occur. Rather, what occurs, as explained elsewhere in this article, is first there will appear to be two quantum atoms that are about to contact or touch, and then upon touching, there is one. Touching and contacting is never in fact witnessed; only amalgamation is.

${ }^{131}$ Other philosophers also come to this conclusion, such as Lange (2002, 6-7).

篮 Springer
} 
not identical from the single improper part), it however has a mereological structure and thus has an individuality (haecceitism) that is distinct from $\mathrm{A}_{2}$ 's. ${ }^{132}$ If this is the case, the haecceitism of $A_{1}$ and the haecceitism of $A_{2}$ allegedly would remain distinct upon the coincidence of points $A_{1}$ and $A_{2}$, and thus $A_{1}$ and $A_{2}$ can allegedly share a part-whole relation upon their coincidence thus giving rise to a composite point-sized item that is "more than" either of the point-parts $A_{1}$ or $A_{2} \cdot{ }^{133}$ But this objection is of no avail, since the haecceitism itself must contribute to the contacting of $\mathrm{A}_{1}$ and $\mathrm{A}_{2}$ : the haecceitisms of $\mathrm{A}_{1}$ and $\mathrm{A}_{2}$ are what are actually doing the contacting or touching: if the points really contact, it must be that the thisnesses of these items are that contact. Now these haecceitisms are surely without haecceitisms of their own, lest an infinite vicious regress ensue (the steps to complete the task of providing thisness to either $\mathrm{A}_{1}$ or $\mathrm{A}_{2}$ is never completed). Therefore, if it is alleged that the haecceitisms of $A_{1}$ and $A_{2}$ remain distinct during the contacting of $A_{1}$ and $\mathrm{A}_{2}$, these haecceitisms, which are haecceitismless points, and which are partless and unstructured, appear to involve the contacting of undifferentiating onenesses, and thus appear to unify and become identical upon contacting, given the reasoning in the previous paragraph about the contacting or touching of undifferentiated onenesses.

A philosopher may wish to object and to maintain that thisness is not actually the item doing the contacting or touching, but rather it is a property that the item has, and thus it is not the thisnesses that contact, but rather it is the items that have the thisnesses that contact. This account is a bare particular account, since this account requires us to consider properties to be distinct from their property possessors. ${ }^{134}$ (If they weren't distinct, we would again be talking about the touching or contacting of thisnessless thisnesses.) And on such an account, then if the point-sized items touch or contact, it would be a "touching" or "contacting" of two bare particulars. But the touching of two bare particulars-where we are really considering only a bare (propertyless, ineffable) item, the so-called propertyless "inner nature" 135 of a substance-is merely the touching of two undifferentiated onenesses, which cannot give rise to a composite since they would only amount to one undifferentiated

\footnotetext{
${ }^{132}$ French describes thisness as follows:
}

Alternatively, the individuality of an object has been expressed in terms of its 'haecceity' or 'primitive thisness' (Adams 1979). As the name suggests, this is taken to be the primitive basis of individuality, which cannot be analysed further. However, it has also been identified with the notion of self-identity, understood as a relational property (Adams ibid.) and expressed more formally as ' $a=a$ '. Each individual is understood to be identical to itself. This may seem like a form of the property-based approach we started with, but self-identity is a rather peculiar kind of property. (2006, section 2)

${ }^{133}$ Only the mereological relation would make the whole more than the point-parts. But recall in Sect. 3 how I mentioned that it is unclear how this composite is more than its parts. Only the relation is more than the parts, but how is the relation not a constituent of the whole? It seems it is since it fits all the criteria: contributes to the make up of the whole (just like any of the other parts), coincides with the object in question (just like any of the other parts), and if it did not exist, the whole would change (or go out of existence) since it would go from being $\mathrm{W}_{1}$ to $\mathrm{W}_{2}$ (just like any of the other parts). If the relation is merely a constituent of the whole, then this leads to whole $=$ three (and the rejection that whole > parts), which is the eliminativist position.

134 The alternatives to this thin/bare particular account are the thick particular account or the bundle theory, both of which I attack in Grupp (2006a).

135 Moreland (2003, 3-4). 
oneness, as discussed two paragraphs back. (These issues are discussed in much more details in Grupp (2006a).)

The reasoning of this subsection and of 4.2.2.1 reveals that if there is to be touching, or contacting between items, such as atoms, they have to have a surface by with to touch other items. And in order to have a surface, they therefore must have an extendedness (magnitude). I will next discuss atoms that are extended. At other places in this article, and following others, I have called atoms that are extended Democritean atoms (rather than calling them abstract [immaterial] atoms, which would not make sense if extended objects are material objects). In order to discuss touching between atoms we must discuss Democritean atoms. Democritus's atoms (somehow) have an irreducible size (an indivisible, uncuttable and partless spatial magnitude) and thus a surface called Democritean atoms. The only way atoms can touch is if they have magnitude and thus a surface.

\subsubsection{The impossibility of atoms contacting, touching, attaching, or abutting: an argument against democritean atoms}

4.2.3.1 The impossibility of non-coinciding atoms contacting, touching, attaching, or abutting The Democritean position for quantum atoms appears to be, from what I can tell, less widely held than the point-particle position. But it has a reasonable following, and it seems to be something like the position held by Planck-scale physicists hold. To give an example of philosophers who advocate the Democritean position, consider a passage Hoffman and Rosenkratz, who argue for this position:

...there are sufficiently strong empirical reasons to affirm the existence of voluminous atomic particles, for example, Democritean atoms in the form of electrons and photons. We doubt that there is at present any good empirical evidence for the existence of point-particles. ${ }^{136}$

In this section, I will assume that there are such Democritean quantum atoms in order to attack the position, showing that the contacting or touching between such supposed atoms is impossible (we don't need to worry about their interconnectedness given what was argued for in 4.3). I will carry out my attack by presenting a novel attack against Democritean quantum atoms which shows that they do not exist.

\subsubsection{Half-less spheres ${ }^{137}$ Consider the following two statements which describe a Democritean atom:}

S1: There are no pieces of the atom that are smaller than the entirety of the atom. S2: There is only one (improper) part of the atom, and that is the atom's entirety.

\footnotetext{
136 Hoffman and Rosenkrantz $(1997,151)$. Hoffman and Rosenkratnz attempt to describe a Democritean atom (extended atom) as having parts by defining a new concept of parthood, and where they call the "parts" of the atom non-substances (51-52). But from what I can tell, it is merely an ad hoc attempt to get around problems to do with extended atoms, and at any rate, if one merely applied the reasoning given in this paper about extended atoms to their "composite atoms," that would only reveal their "non-substance" parts of their atoms to be in fact real parts. Also, the reasoning in Sect. 3 of this article would do away with such "composite atoms."

137 The material in this first subsection (4.4.1) was developed to some degree with Christian Nallenweg of Indiana University Northwest.
} 
Even though an observer can see with her eyes that there are sections of the atom (if she could see extended atoms), such as a top and bottom, nevertheless there is nothing smaller than the atom. If statements S1 and S2 are true, then there are no real items that are referents of the following statements, "half of the atom," "one quarter of the atom," "a point inside or on the surface of the atom." Imagine that the atom is a sphere (what shape the atom is, is irrelevant here, and any shape could be considered). If it is, then it is a sphere that does not have, for example, two halves, if statements of the sort just given ("half of the atom," "one quarter of the atom," "a point inside or on the surface of the atom.") do not have referents. If the halves or hemispheres are merely mental concepts, and not real elements of the Democritean sphere, then we have a sphere without halves or hemispheres. But a sphere (since we are considering the atom to be a sphere at the moment) without real halves or hemispheres is not a sphere at all. Another way to put the matter is as follows. We cannot think of a partless, halfless, and hemisphereless sphere as having a radius, due to the fact that a radius is a ray drawn from the center (which is a part that it seems any sphere must have) to the edge of the circle, and if we are not allowed to talk about the parts of the sphere, then we cannot mention the center of the sphere from which the radius can be considered. So if S1 and S2 are the case, then we have a radius-less sphere, which is an absurdity. None of these parts-center, radius, half, hemisphere, etc.-of the sphere exist and none of them can be discussed, but if that's the case, this is not a sphere at all, and a spherical Democritean atom is impossible. Similar reasoning could be given for Democritean extended atoms that have other shapes.

\subsubsection{Sizeless Distances But is a radius, center, or hemisphere a real proper part of} the sphere? Or are these just concepts in the mind, representations of how a person might think about a sphere, including a partless Democritean quantum atom that is theorized to be the shape of a sphere? An observer could clearly see halves, a center, or quarters and so on of the Democritean quantum sphere (if one could see inside the Democritean sphere, such as if it were semi-transparent), but in the end it may be that they are no more than the mind erroneously clumping off bits (imaginary parts) of the atom-bits that do not exist-assuming they are parts when they are not. Another way to put this objection is as follows: If there are partless, extended atoms, one can't use mathematical concepts, since (most) mathematical descriptions will involve any objects (spheres, triangles, fractals, etc.) that are composites. (For example, in topology extension is considered to be a continuum of interrelated points, where the points are parts.) So radius, half, hemisphere, etc. correspond not to actual parts or the atom, but to thoughts one might have about the imagined parts of the atom. According to this objection, it is the use of language, using terms such as "radius" and "half," that make it appear that the Democritean quantum atom has segments that can be discussed, but if we merely choose different set of statements to describe the atom, the parts talk does not emerge, such as these statements describing the Democritean atom. Critics of the argument against Democritean atoms just given above will therefore likely argue that I did not discuss an actual Democritean atom since I described the atom in mathematical terms. Rather, objectors may assert that I am discussing my ideas about what the Democritean quantum atom is like, which do not denote any mind-independent characteristics of the Democritean quantum atom; I am creating a false idea of what the Democritean atom is like and attacking that idea. Rather, I must describe the Democritean atom non-mathematically and in a way where no person can conceptualize it to be other than an undifferentiated oneness. If this objection is correct, I must 
consider a Democritean atom without using the terms "radius," "half," "left side," "right side," and so on.

I will next discuss serious problems with Democritean quantum atoms which do not involve any mathematical terms whatsoever, and which can be articulated only in terms that involve the Democritean atom as being an undifferentiated oneness. I will only use the concepts of size and distance. Size is the hallmark of a Democritean atom. I will consider "size" to not strictly be only a mathematical term. I will consider size also to be given in terms of distance, and I will also explain why "distance" is a pre-philosophical term, rather than a mathematical term, and thus one that I can use which avoids the above objection.

Before getting to these terms, and before explaining why they are not mathematical terms, first I will explain as briefly as possible what I will do in this section to argue that Democritean quantum atoms do not exist. Democritean atoms have size, and I will argue that the concept of size can only be coherent if two objects do not overlap and are at a distance from one another-call them seg1 and seg2-but then I will show that any Democritean quantum atom cannot have seg1 and seg2 and thus cannot have size: there are no Democritean quantum atoms.

Distance is a concept apprehended in the mind regarding items that do not perfectly coincide. The mind apprehends separate bits of matter, and by their being non-collocated, they are then believed by the mind to involve a third element: a relationship of distance between the bits of matter. (Metaphysical and other realists

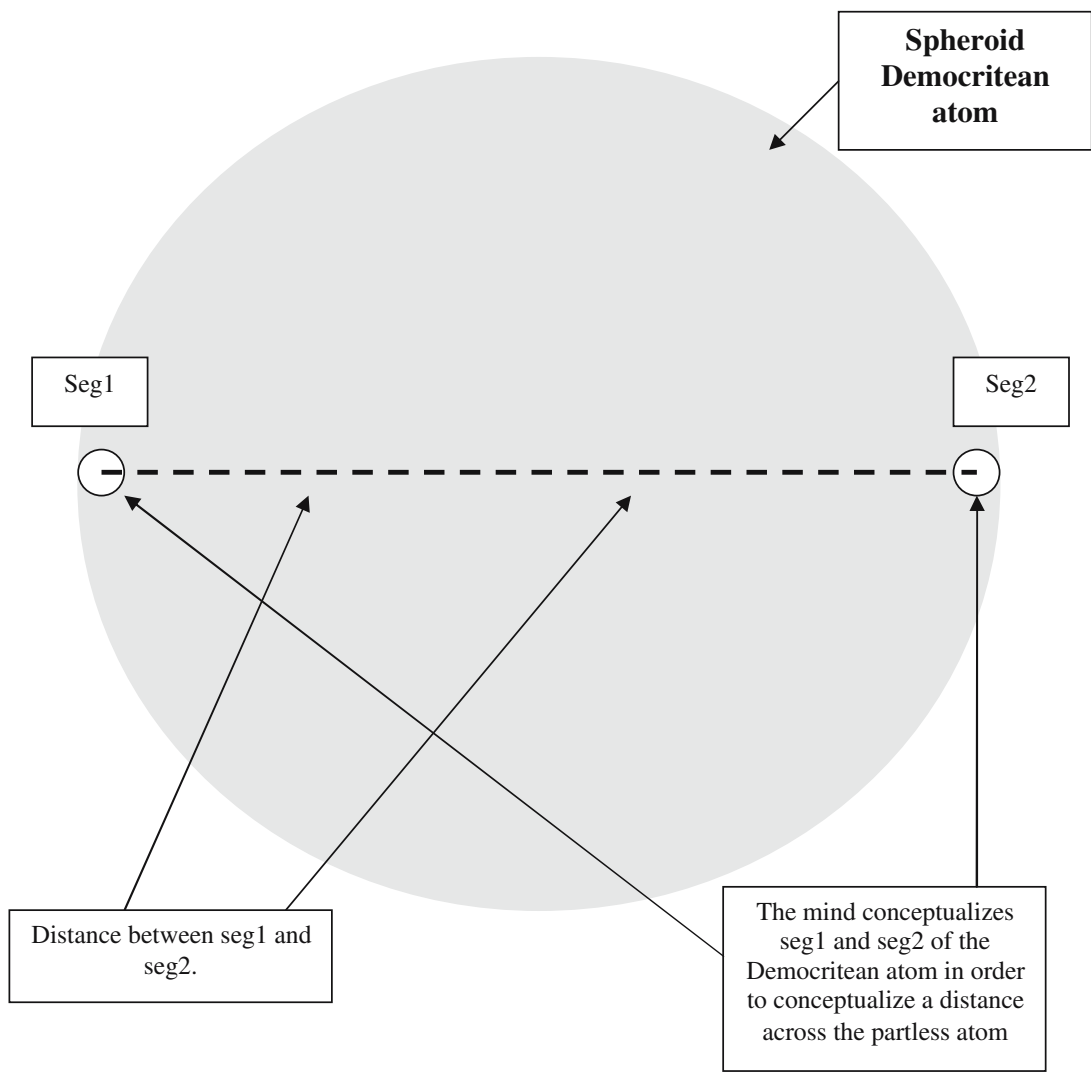


will likely maintain that this relation is a real mind-independent item in reality interrelating the non-coinciding items.) For these reasons, if a Democritean atom involves distance-which it must since it has size-the mind must be able to conceptualize bits of the undifferentiated oneness (the atom)-even if they are only ways of thinking about the Democritean quantum atom, and not real proper parts of the atom-in order to consider distance, and therefore size. If there are no bits of the undifferentiated oneness that the observer can witness, and witness them as if they are at a distance from one another, in order to perceive distance and size-i.e., if all perceivable and conceptualized regions of the undifferentiated oneness that the mind can consider when observing the atom, even though they are not real parts, but are conceptual parts - then there is no distance between the "bits" of the undifferentiated oneness. So only if there are conceptually separable bits of atom to consider, there is no way to theorize that the undifferentiated oneness has any distance.

Some philosophers may object to this definition of distance, since there certainly can be distance when there are no objects present, such as in the void of space where there are cubic meters with no particles. But this is nevertheless a definition of distance in terms of objects, and the objects in this case are points of space. It just happens to be empty space (space without any occupants), and is therefore distance between objects.

Next, let's define the concept of size as follows: any item, such as an atom, has size if it is not a point atom, and if it has an extension that has a distance across it. (Any definition of distance-metaphysical realist conceptions, etc.-will do for my purposes of this argument below.)

My objectors will likely maintain that these seg1 and seg2 are concepts, and not real physical parts of the atom, since, regardless of whether or not the mind can conceptualize bits of the atom that appear at a distance from one another, there is nothing real that corresponds to them, and such concepts are erroneous and cannot generate theories about real existent items. I of course cannot object; but this only helps my case, for the following reasons. The problem is this: if that is the case, and there are no real mind-independently existing seg1 and seg2 parts of a Democritean quantum atom, then it leads to the conclusion that there is no distance across the Democritean quantum atom. Above we saw that distance requires that there be items that actually exist in a non-collocated manner, wherein there can be spaciousness between them. But if a Democritean atom does not have such parts existing in a noncollocated manner, then a Democritean atom does not have distance and therein no size: it is a point. The imagined seg1 and seg2 do not exist since there is only the whole, which is one improper part that itself has no parts. If that is the case, then there are no items that can be considered to be at a distance from one another, and without those, we can only consider the distance and size to do with the atom to involve nonexistent items that are at a distance from one another, which is an absurdity-analogous to discussing the distance between two round squares. If what has been written is correct, I will next discuss that another statement (S3) must be false:

S3: There is a real mind-independent span of size across the Democritean atom, since size involves distance.

S3 is false is because distance, as just defined, involves bits of "stuff" that does not coincide, but a Democritean atom is undifferentiated and there are no 
distinct stuffs "inside" of it. If that is the case, then there is nothing "within" the Democritean quantum that is at any distance from anything else, and thus a Democritean quantum atom cannot involve distance across it-hence S3 is false. In other words, to have real distance, there must in fact be distinct bits of "stuff" not collocated, but a Democritean quantum atom, does not involve any distinctions whatsoever. There are no differentiated "stuffs" that can be described as "at a distance from each other" in a Democritean quantum atom since the Democritean atom is only one undifferentiated being. Since there is no way to define distance at all with respect to a Democritean quantum atom, then the next statement (S4) follows:

S4: There is no mind-independent distance across the Democritean quantum atom and thus the Democritean atom has no size (and is a point)

I will next address a few more objections to the reasoning given in this subsection. The argumentation rests on their being distinguishable items that are at a distance from one another. Even though the Democritean atoms is supposed to be a seamless undifferentiated oneness, perhaps there could be indistinguishable bits the Democritean atom that are at a distance from one another, just as quantum abstract atoms can be indistinguishable but not coinciding. This objection however clearly fails since there cannot be bits of the Democritean atom "inside" of the atom, whether they are distinguishable or indistinguishable. (In the next subsection I will ignore this and imagine that somehow Democritean atoms can have indistinguishable innards in order to further explore this objection.)

Another objection can be goes as follows: Why must we define distance in terms of items? Why can't we merely define distance as the mere irreducible expanse across the undifferentiated oneness of a Democritean atom, where its distance is just that very oneness? On this account, distance is defined in terms of unstructured, primitive expanse of oneness, rather than in terms of the non-coincidence of, or the expanse between, some item $\mathrm{p}$ and some item q. This objection avoids reference to any "bit" of the Democritean atom that is not the identical to the entirety of the atom, or to the "inside" of the Democritean atom, as all of the above accounts did. On this account, a Democritean atom just has an irreducible size, no reference to innards is made, and there is nothing more to assert than that.

This account may involve problems, however. As just maintained, the specific point of this objection is to maintain that it is an account of distance that does not involve distance between or among any two objects. If this is the case, then, distance here is not distance between or among any items, which takes us back to the original problem given above regarding the distance between seg1 and seg2 of a Democritean atom: this distance is not between any two items, but since it is a distance, it is thus is a distance between non-item and non-item-a "distance" between nothings. How can nothing be at a distance from nothing? What can be said about nothing? There is no sentence that can denote it, and thus maintaining that nothing is at a distance form nothing is to give nothing properties, which would indicate that it is something, not nothing - and that is a contradiction. Furthermore, nothing is nowhere, and is thus not proximate to anything, and nothing is not at any distance, or next to, or beside, or 8 light years from, anything at all. According to this objection, there appears to be no distance at all if the objection involves the position that nothing is distant from nothing. 
4.2.3.4 An objection: entendedness I will next discuss an objection proponents of Democritean atoms may convey. Consider the concept of entendedness, as defined by Parsons:

Entended $={ }_{\mathrm{df}}$ an object $\mathrm{x}$ is entended if " $x$ is a material object that is wholly and entirely located at a non-point-sized region, $r$, and for each proper subregion of $r, r^{*}, \mathrm{x}$ is wholly located at $r^{*} ., 138$

The reason I bring this up here is because some philosophers may imagine that a Democritean atom apparently is an entended object, and if it is, it certainly would get around the arguments above. Entendedness may be considered an attractive philosophical principle for Democritean atomism because with it we may discuss "regions" of a Democritean atom, and therefore distance across a Democritean atom, without S4 ensuing.

In what follows I will show that the concept of entendedness is impossible. Following Parsons' definition of entendedness, a Democritean atom, D, can be considered to be entirely located at the topological region, $\mathrm{T}$, it is located at; call the atom from this perspective $\mathrm{D}_{\mathrm{A}}$. Also, $\mathrm{D}$ can be considered to be entirely located at any of any point-sized locations of the $T$; call the atom from this perspective $D_{P}$. Also, D can be considered entirely located at one quarter of T; call D from this perspective $\mathrm{D}_{\mathrm{Q}}$.

There are several problems here. First, notice that if we follow Parsons' definition of entendedness, then $\mathrm{D}=\mathrm{D}_{\mathrm{P}}=\mathrm{D}_{\mathrm{Q}}=\mathrm{D}_{\mathrm{A}}$. Now notice that $\mathrm{D}_{\mathrm{P}}$ is unextended, and $\mathrm{D}_{\mathrm{A}}$ and $\mathrm{D}_{\mathrm{Q}}$ are extended. But if that is the case, then to maintain that $\mathrm{D}_{\mathrm{P}}=$ $\mathrm{D}_{\mathrm{Q}}=\mathrm{D}_{\mathrm{A}}$ is to maintain that a point-sized object $\left(\mathrm{D}_{\mathrm{P}}\right)$ is identical to a non-point-sized object $\left(\mathrm{D}_{\mathrm{Q}}\right.$ or $\left.\mathrm{D}_{\mathrm{A}}\right)$. But if a point-sized object, in this case, $\mathrm{D}$, is identical to a non-pointsized object, then $\mathrm{D}$ is point sized $(\mathrm{P})$ and not point-sized $(\sim \mathrm{P})$, and thus $\mathrm{D}$ is $\mathrm{P} \wedge \sim \mathrm{P}$, which is to say that an unextended atom is an extended atom, and thus is an absurdity. Therefore, $\mathrm{D}_{\mathrm{P}}$ is not a possibility for an entended object. But in considering $\mathrm{D}_{\mathrm{A}}=\mathrm{D}_{\mathrm{Q}}$, more problems ensue. Imagine that $\mathrm{D}_{\mathrm{Q}}$ is some length across, call that length $\mathrm{L}_{\mathrm{Q}}$. (Above I proved that there cannot be any atoms with length or size, but that reasoning does not apply to entended atoms, and that is why I am considering atomic size or length here). Now imagine that $\mathrm{D}_{\mathrm{A}}$ is some larger length across, call that length $\mathrm{L}_{\mathrm{A}}$. Now $\mathrm{L}_{\mathrm{Q}} \neq \mathrm{L}_{\mathrm{A}}$, but $\mathrm{D}_{\mathrm{Q}}=\mathrm{D}_{\mathrm{A}}$. This leads to the conclusion that $\mathrm{D}_{\mathrm{A}}$ is both $\mathrm{L}_{\mathrm{A}}$ and is $\mathrm{L}_{\mathrm{Q}}$ across. This means that the quarter entended Democritean atom, $\mathrm{D}_{\mathrm{Q}}$, can travel through a hole that is length $\mathrm{L}_{\mathrm{Q}}$ wide, but the whole entended Democritean atom, $\mathrm{D}_{\mathrm{A}}$, cannot travel through the hole that is length $\mathrm{L}_{\mathrm{Q}}$. In other words, Democritean atom $\mathrm{D}$ can and cannot travel through the hole in question, which is a contradiction. For reasons such as these, I leave the concept of entendedness, considering it not to be a viable description of any Democritean atom. ${ }^{139}$

\footnotetext{
138 These are the words of Josh Parsons. I took this definition from Hud Hudson's forthcoming book Hyperspace, which he presented a chapter of at Purdue University in November 2005, where Hudson's proof maintained that Parson's description of 'entendedness' is unpublished. Parson's online CV however indicates that the concept of entendedness has appeared in a book chapter in an anthology.

139 Some may imagine that the new theoretical work in string theory can avoid this idea that there are no surfaces or colors that can be composed of quantum objects, since strings are not points, but are 1-dimentional items, and some strings theorists now theorize the existence of 2-dimensional sheets. As mentioned in a note above, strings are basic building blocks that have a 1-or 2D extension, and thus it appears that they are impossible, given the evidence just presented against the existence of any items that are partless and extended (1-, 2-, 3-, ... $n$-dimensional).
} 


\subsection{No relations or connections between quantum abstract atoms}

The reasoning given above in this subsection also reveals that there are no relations between quantum abstract atoms. Relations must link (to used Loux's word) to their relata (if they did not, they would not do the task of connecting them to one another). In order to link, the relation and the relata, we are told by metaphysicians, must have some sort of special contacting capacities-a contacting that metaphysicians typically assert to be primitive and unanalyzable so as to avoid Bradley's regress. Whether or not this alleged primitivism allows the relatarelation linkage to avoid the problems of Sect. 4.2 above is not clear. But that appears to however be irrelevant when we consider what any metaphysical relation, if it existed, can and cannot do (when we consider the capacities any alleged metaphysical relation can and cannot have). A relation, $\mathrm{R}$, either has a surface or it does not. It if does, it is susceptible to the problems of 4.2 .3 , if it does not, to the problems of 4.2.2. So the alleged primitivity does not even come into the picture if we merely ask: What can the relation touch and not touch? And it appears that it cannot touch anything at all-whether a bare particular, a thick particular, and so forth-since it cannot do so with or without a surface. Therefore, relations have no way of linking to their relata, and relations cannot relate. (In Sect. 5.1 I will discuss many additional fatal problems to do with relations (none of which I have presented elsewhere).)

The philosophical position that there are no relations between atoms has been in the past a mainstream traditional atomist position. There have been specific attacks against the existence of such relations put forward by some of the ancient Indian Buddhists philosophers, such as Dharmakīti, ${ }^{140}$ more recently by Grupp, ${ }^{141}$ and I suspect it is an aspect of Quine's philosophy of propertylessness (since relations are relational properties). If any of these attacks are correct, then there are no mereological relations, which would mean that the only way we know how to describe a part as being a part, or a whole as being a whole-which is by using mereological relations-is misguided, and therein we have no way to describe how parts and whole exist. In other words, regardless of what we think we believe about reality from what our senses tell us, it would instead be the case that reality contains no parts and wholes whatsoever, and there are no macroscopic and empirical items.

The theories of Western philosophy and of scientific theory (except the standard model of quantum mechanics, see below) ultimately describe all things (ordinary material objects, partless fundamental particles building blocks, locations in space, etc.) as being in various relationships to one another. In other words, all theories of science and philosophy in the Western tradition ultimately involve, and are given in terms of, a mereological structure. For example, in chemistry, a DNA molecule is believed to be part of a chromosome; in mathematics, a number is believed to be part of its given number set or number line it belongs to. In ordinary daily life, it is believed that one's self is a part of society, and that one's thoughts are a part of their consciousness. My students are considered to be part of the university, and the sun is believed to be part of the galaxy. In the examples of the DNA strand, the self, the students, and the sun,

\footnotetext{
140 See JHA (1990, 13 and 17).

141 Grupp (2005a, b, c, d, 2006a).

型 Springer
} 
the mereological associations and mereological relations believed to exist in those cases involve the interrelatedness of non-collocated objects (objects that are not directly touching). There is really no other way to describe parthood nonmetaphysically. The question is: what is it that makes one item part of another? How is it that any item can be a part of any other item? The only way to answer these questions is to espouse a philosophy of relationships, where relations connect objects (parts and wholes), and where these connections give rise to reality. If it were found that these relations are impossible, then it would be fair to say that the best theory of reality we have is one where reality is in fact devoid any parts and wholes. In other words, regardless of what we think we believe about reality from what our senses tell us, it would instead be the case that reality is wholeless, since there would be no theory (philosophical or scientific theory) describing in a non-contradictory way how parts and wholes can exist, and since our science (quantum mechanics) also points to the non-existence of connections between subatomic particles. This is the conclusion I will arrive at in this article. But first I will discuss the history of relations and the various sorts of metaphysical connections that have been theorized to exist, but which I will show do not exist, but which I will show do not exist.

Philosophers have long maintained that interaction between quantum abstract atoms need not occur by touching, but rather via interrelating, or by mediating forces. For example, Kline and Matheson writes:

Absolutely no one still believes that every physical interaction consists of material bodies bumping into each other. Those who have tried to work out a completely mechanistic physics have been unable to explain common phenomena like liquidity, gravitation and magnetism. ${ }^{142}$

Kline and Matheson also tell us that to reject collision by touching "is to subscribe to some sort of field theory. ${ }^{143}$ In the era of classical physics, which was roughly from 1680-1900, this framework persisted, and Herbert shows us just how important the concepts of fields and forces are:

Classical physicists were able to account for all the world's variety by means of only two physical entities - matter and fields. In those innocent days it went without saying that these entities were really there. Physicists' reality crisis was yet to come.

Real matter. Real fields. Drop an apple from a bridge. The apple is made of matter. It moves because the Earth's gravitational field pulls it. Everything in the world works the same way: matter produces force fields, which move other matter...

Classical physics in a nutshell: The universe consists of nothing but matter and fields-and we know the laws of both. ${ }^{144}$

Continuous (unbroken, non-particulate) fields, forces, relations, and connections between atoms, and which act as intermediaries between atoms, keeping them from directly touching, could be theorized to exist by philosophers in order to avoid the problems of touching and contacting. The issue at stake is this: Is there another means

\footnotetext{
$\overline{142}$ Kline and Matheson $(1987,509)$.

143 Ibid., 510.

144 Herbert (1985, 32-34).
} 
by which atoms can interact and/or connect other than merely by touching, contacting, and abutting directly? It appears that if there are forces, relations or connections between atoms, acting as intermediaries between the atoms, ${ }^{145,146}$ then the atoms

\footnotetext{
145 To see why I am lumping together all these concepts-force, field, relation, connection-see Jammer, Concepts of Force, New York: Dover, where he shows us how these concepts all perform similar functions, and are described interchangeably at times. The history of force relations is long, starting, unsurprisingly, with the Greeks. Jammer writes:
}

Force as a regulative agent in nature appears, perhaps for the first time in Greek thought, in Empedocles' doctrine of love and strife, and in Anaxagoras' theory of the mind (nous). Both doctrines aimed at an explanation of the causes of motion... These agents as causes of motion may rightfully be interpreted as "forces", although they were not held as immaterial, but as extended in space and corporeal. (Jammer 1999, 25.)

Plato interpreted Empedocles' two agents as attraction and repulsion, stating that their operation is conceived in an alternative sequence, whereas, according to Plato, the same forces operate simultaneously in Heraclitus' conception of nature. (Jammer 1999, 27.)

...Aristotle recognizes two kinds of forces, the Platonic conception of force inherent in matter, which he calls "nature" (physis), and force as an emanation from substance, the force of push and pull, causing the motion in a second object, and not in itself... For his mechanics, Aristotle confines himself solely to the concept of force as the agency involved in pulling or pushing, and ignores the Platonic concept of force as inherent in matter or what we may call today energy. (Jammer 1999, 35-36)

In his search for possible other phenomena in which the attractive force of the sun may become perceptible or demonstrable, Kepler was thus left with only one possibility: Gilbert's magnetic forces. No wonder that Kepler, when writing his Tertius in terveniens, is convinced beyond any shade of doubt that his astronomical computations only confirmed his previous assumptions about the importance of magnetic forces. In article No. 51 of this short treatise Kepler asserts emphatically: "The planets are magnets and are driven around by the sun by magnetic force."...

Kepler imagined these magnetic forces, emanating from the central body such as the sun, to be like giant arms propelling the planets on their appropriate orbits. He thought it necessary that for this purpose the central body itself should be in rotational motion. (Jammer 1999, 89-90)

146 To my knowledge, nowadays forces and fields are described completely in terms of particles, which will be significant to the reasoning of this paper. Kane writes:

Past study has led to the establishment of the Standard Model of particle physics, a complete description of the basic particles and forces that shape our world... The world we see is built entirely of three particles: the electron and two particles similar to the electron called quarks... [T] he Standard Model is not expected to be the final stage of particles physics, even though it successfully describes phenomena and experiments... (Kane 2000, 16)

The picture of the electromagnetic force [for example,] that emerges is that electrons and any particles that have electric charge, interact by exchanging photons. The photons carry energy between the electrons; two electrons can scatter off one another by exchanging a photon; and an electron and a proton bind by exchanging many photons, which provide an attractive force that keeps the electron and proton connected in a stable objects, a hydrogen atom. All the forces work in a similar way. The gravitational force arises from exchange of gravitons. The analogous particles for the weak interactions are called $\mathrm{W}$ and $\mathrm{Z}$ bosons, and for the strong force they are called gluons. In all these cases, we speak of the photon, $\mathrm{W}$ and $\mathrm{Z}$ bosons, and gravitons as mediating the forces. (Kane 2000, 18) 
could indeed interconnect or interrelate without touching or contacting, and thus there is a possibility that atoms can give rise to a composite object while avoiding the problems of touching and contacting. This sort of touching, it seems, could be looked upon favorably also by philosophers who endorse the philosophy of point-particles. Point-particles do not have surfaces, and I know of no argument or description in physics or philosophy that reveals how surfaceless items such as point-particles can directly touch one another without a mediating metaphysical relation or interconnection that connects them. Further, it may be hard to imagine how point-particles could touch anything at all, due to the fact that they do not have surfaces, and it may be hard to imagine how relations can touch anything at all, but since this sort of "touching" or linking could merely be a different sort of touching (often called instantiating, or exemplifying) between relation and relata, many may endorse this position. ${ }^{147,148}$

Many philosophers and physicists reject that there is touching and direct contacting between quantum abstract atoms, but freely maintain that there are instances of direct contacting or touching between quantum abstract atoms and fields or forces, or between quantum abstract atoms and relations or metaphysical connections, wherein fields or forces or relations or connections are continuous (unbroken, nonparticulate) intermediaries between the quantum abstract atoms. Typically when this move is made, no description is made as to how it is that there can be a touching interface between these items with the quantum abstract atoms without the same sorts of problems of contacting and touching between quantum abstract atoms emerging. ${ }^{149}$ (The Kline and Matheson passage above is from an article that provides a good example of this.)

But no attempt to justify how these items can serve as continuous (unbroken, non-particulate) interconnections between quantum abstract atoms would be needed since above I showed that there cannot be any composite items, and thus no composite intermediaries connections (section 3); and that there cannot be extended non-composites, and thus no extended non-composities intermediary connections (subsection 4.2.3); and thus the only unextended intermediary connections would be left to consider would be unextended intermediary connectinos, but I showed that they cannot touch any quantum abstract atoms, since that would be alleged pointpoint touching, which I showed is impossible (subsection 4.2.2). Theses findings show that such interconnections of any sort-fields, forces, relations, topological connections, etc.-are all impossible. The reasoning given attacks any mereological relation, any connection between particles, and any sort of intermediary items that allegedly bind two items together that are not directly touching.

\footnotetext{
147 Speaking specifically about the metaphysical relations that philosophers often assume exist between particles, the "touching"- or rather the linking (to use Michael Loux's terminology (Loux 1998, 38-41.)) of relations to the items (relata) the relations interconnect-between relation and point-particle must be analogous to the touching of two non-physical point-items (see Grupp 2006a), which I show is impossible if the contacting items are to keep their individuality when they are "touching.",

148 Grupp (2003, 2004a, b, 2005b, c, 2006a) offer new arguments against the idea that any item can instantiate and exemplify any property.

149 Typically the interfacing or continuous integration of fields to quantum abstract atoms, or forces to quantum abstract atoms, relations to atoms, and so forth, is not discussed. This could hide the fact that the forces, fields, and relations lead to problems in their alleged contacting or touching with the quantum abstract atoms - if there is any such contacting and touching (quantum physicists who are aligned with the Standard Model routinely reject any such contacting or touching, but philosophers seem to think there is).
} 


\subsection{Action-at-a-distance}

An obvious way to avoid all the attacks against relating and contacting between quantum abstract atoms (or between any DIV2 items that the ordinary empirical consciousness can conjure up) given in Sects. 4.2 and 4.3 is by merely rejecting locality, and by asserting that there is action at a distance (AD) between particles. What it means to assert that locality can be violated is to maintain that object $\mathrm{A}$, which is located only where it is and nowhere else, can interact with another item that is not where A is located at, and without touching or contacting the other item. This would be to say that, for example, I can have a pen on my desk that can have an interaction with a book in the next room, while each book and pen remain where they are, without one going in the other's room to interact with each other. ${ }^{150}$ In Sects. 4.2 and 4.3 above, the assumption was made that entities can only involve interaction where they are, and not somewhere else. In other words, this is like maintaining that a lion that is in Africa standing near a zebra can only have interaction with the zebra if the two of them move toward one another and coincide or touch (in the DIV2 sense), such as when the lion attempts to eat the zebra. It seems absurd to maintain that the lion, which is in Africa, can have interaction with the zebra where the interacting with the lion is not where the lion is, and is instead somewhere else: only where the zebra is and not where the lion is, or only in Chicago, or only on Alpha Centauri. On this issue, Samuel Clarke wrote: "That one body should attract another without any intermediate means is indeed... a contradiction: for "tis supposing something to act where it is not." ${ }^{151}$ Lange mentions that this is only true if locality is assumed. ${ }^{152}$

Although philosophers who are not familiar with quantum physics and who are ingrained with classical concepts (discussed above) and something like a mechanistic philosophy of DIV2 consciousness may find nonlocality antiquated and passé, on the contrary, following the work of the physicist John Bell, it appears that physicists have discovered that quantum reality is irreducibly non-local, ${ }^{153}$ and it is the classical concepts and the DIV2 mechanistic and causal philosophy that is passé and antiquated. ${ }^{154}$ Further, the impossibility of contacting and touching, which are localistic concepts, are philosophical issues that quantum physics draws into serious question and which I above showed apparently do not exist. In Sect. 4.3, the purpose of introducing a field, and/or relations and connections to act as intermediaries between particles was to keep the activity between particles local, since contiguity and contacting between fields and/ or relations/connections on the one hand, and the quantum abstract atoms, on the other, remains. As discussed above, fields merely leads to more problems, and from the perspective of quantum mechanics, it is the idea of continuous field connectedness that is antiquated, not AD. (This is discussed much more in Sect. 5 below.)

\footnotetext{
150 See Lange (2002), chapter 1, especially pp. 13-15. Also, Maudlin's work is considered a classic on quantum non-locality.

151 In Alexander, H.G. 1956. The Leibniz-Clarke Correspondence. Manchester: Manchester University Press. Page 53. Cited in Lange (2002, 95).

152 Lange (2002, 95).

153 See Nadeau and Kafatos (1999).

154 As of 2006, to my knowledge, physicists have little knowledge of this multi-decade-old revolutionary discovery in quantum physics. This is a good indication of how much philosophers have distanced themselves from the progress of quantum science.
} 
From what I can tell, AD avoids all the problems discussed above, which all involve localistic descriptions. Non-locality allows for "interaction" of some sort, even if at a distance, which is merely an interaction that is not contacting and/or direct touching: interaction between objects that are not contiguous, but they are nevertheless believed to interact. Sections 4.2 and 4.3 above put strict limitations on how AD can occur. Philosophers and physicists who do discuss AD describe it as some sort of a relation. ${ }^{155}$ The findings in 4.2 and 4.3 above show that AD-interaction cannot occur via touching, contacting, relating or connecting. Therefore, AD would only occur by some utterly unknown and mysterious process, ${ }^{156}$ which makes proceeding uncomfortable, but I will just so I can given an argument that appears to show that action at a distance involves serious problems.

If quantum abstract atoms $\mathrm{A}_{1}$ and $\mathrm{A}_{2}$ are involved in $\mathrm{AD}, \mathrm{A}_{1}$ can act where it is not, and $A_{2}$ can act where it is not, to put it in Clarke's wording. This would mean that $A_{1}$ and $A_{2}$ that are next to me, are in fact interacting eight feet away, or eight light years away. I will refer to this proximity I have with $A_{1}$ and $A_{2}$, where $A_{1}$ and $\mathrm{A}_{2}$ are, as here; and I will refer to their interaction eight feet or eight light years away as there. $A_{1}$ and $A_{2}$ are here, but do not interact here. $A_{1}$ and $A_{2}$ interact there, even though they are not over there. The problem I see with $A D$ for $A_{1}$ and $A_{2}$ is that it is what can be called entityless interaction. $\mathrm{A}_{1}$ and $\mathrm{A}_{2}$ are here, and thus are not there, so their interaction going on there can only be an interaction that does not involve $A_{1}$ and $A_{2}$ being present where the interacting goes on (there). This merely follows from Clarke's comment that AD allows an entity "to act where it is not," and if it does, the action going on does not involve an item that is acting.

P1: $A_{1}$ and $A_{2}$ are here, and are not there.

$\mathrm{P} 2$ : The interaction of $\mathrm{A}_{1}$ and $\mathrm{A}_{2}$ is there, and not here.

$\mathrm{P} 1$ and $\mathrm{P} 2$ reveal that while $\mathrm{A}_{1}$ and $\mathrm{A}_{2}$ are alleged to interact (via non-local means), they however are not present where the interacting occurs. But if they are not, then there is interaction but there are no entities that are interacting: since interaction occurs there but $\mathrm{A}_{1}$ and $\mathrm{A}_{2}$ are not there, ipso facto, interaction is devoid of $\mathrm{A}_{1}$ and $\mathrm{A}_{2}$, and it can be considered entityless interaction. This seems to be an absurdity, as if such a position involves the idea that two non-entities can interact:

\footnotetext{
155 For example, see Nadeau and Kafatos $(1999,3)$. This is one of many that could have been given.

156 AD-interaction may be only a murky issue for AD between distinct (distinguishable) entities, or if it involves interaction at a distance rather than mere correlation via indistinguishability at a distance. (Distance, strictly speaking, does not exist in a mereological nihilist reality, as I discus in this article (distance is given in terms of non-coincidence in nihilism). I merely use it in this footnote for the sake of speaking in familiar terms, and to keep this discussion in the familiar terminology using "action-at-adistance.") In Grupp (2005a) (p. 114) I raised the issue that it could perhaps be the case that the indistinguishable quantum abstract atoms are non-local since if I see atom here (for the sake of brevity, just imagine that an atom can be seen) then it's indistinguishable (literally identical) counterpart there must also have all the same features, and changes in the atom here (such as if it goes out of existence) can only mean there are instantaneous changes in its counterpart there. This can be considered nonlocality via indistinguishability, rather than via a metaphysical relation or some other metaphysical idea. I call this non-locality via indistinguishability, and it is the only mechanism of nonlocality I can think of that is not due to some sort of a metaphysical relation, which is not susceptible to the attacks I present against metaphysics and mereology in this paper, and it is the only one I can think of that is empiricist, since all it requires are indistinguishability of particles (which is an empirically verified for quantum abstract atoms).
} 
nothing $\mathrm{N}_{1}$ can interact with nothing $\mathrm{N}_{2} \cdot \mathrm{A}_{1}$ and $\mathrm{A}_{2}$ are only here, and they are not there, then the interacting that occurs there is interaction without $A_{1}$ and $A_{2}$.

This sort of reasoning applies to any instance where an entity acts where it is not. Consider, for example, a more realistic case than the one just given, where atoms $\mathrm{A}_{1}$ and $\mathrm{A}_{2}$ non-locally interact at a distance and rather than the interaction being entirely not where both $A_{1}$ and $A_{2}$ are (as in the example in the last paragraph), instead the interaction is fully where $\mathrm{A}_{1}$ and $\mathrm{A}_{2}$ are, and it just happens to be AD interaction. But it seems this would still involve entityless interacting. If $A_{1}$ is acting at $A_{2}$ but is not coinciding with $A_{2}$, then the statement " $A_{2}$ interacts with $A_{1}$ " would involve $A_{1}$ not fully being where it is interacting, which is at $A_{2}$. This would give rise to some degree of entityless interaction: $A_{1}$ is non-existent at $A_{2} ; A_{1}$ is not present where the interacting occurs, but is alleged to interact at $A_{2}$. This means that $A_{2}$ is interacting with a nonentity. In other words, if that is the case, then $\mathrm{A}_{2}$ 's having interaction with $\mathrm{A}_{1}$ where $\mathrm{A}_{2}$ is would be analogous to maintaining that $A_{2}$ has interaction with a nothing. ${ }^{157}$

I have apparently shown why no objects at all can touch via AD. This would mean that AD does not occur by touching between particles, or by abutment, attaching, contacting, or by a relation and/or connection between particles. The part-whole relations argued in Sect. 3 to not exist was an attack on relations between items that do not perfectly collocate, and these same sorts of arguments could be run for any sort of relation alleged to exist between objects that are not coinciding perfectly, and thus at the atomic level, all items of reality are unconnected and unattached, implying that they cannot accumulate to give rise to composites of atoms and/or empirical bodies. Therefore, relations and connections cannot account for atoms accumulating to give rise to empirical composite items. This indicates that everything can only be unconnected. ${ }^{158}$

\subsection{Quantum physics does not involve quantum abstract atoms touching}

Contemporary philosophers and physicists often — but certainly not always-freely refer to particle collision as if it is not an incoherent idea. (I suspect that many quantum physicists do this just in order to talk in terms that are familiar to the DIV2 mind that is only aware of classical concepts, such as the concept of surface touching

\footnotetext{
157 Notice that this argument against non-locality only works if A is not indistinguishable to B, and it does not work if $\mathrm{A}$ is indistinguishable from B. As discussed above, quantum abstract atoms are apparently indistinguishable. (I did not state why they are, but it is primarily because if there are no parts and wholes in reality, as mereological nihilism endorses, then there is not way to distinguish any two objects as not being indistinguishable. In Grupp (2006a) I give completely different argumentation that shows why this is the case.) Now it is only in DIV2 that separated, not coinciding objects are considered to be distinguishable; and in DIV1 it is widely acknowledged among physicists that we do not have a clear way to distinguish any particles from any others (See French 2006). So while the argument I just gave seems to show that there cannot be DIV2 non-locality, it does not seem to show that there cannot be DIV1 non-locality. In fact, a primary thesis of physicists is that all quantum abstract atoms may involve non-locality, and that there may not be any non-locally unseparated particles.

158 Some of my previous publications (such as Grupp (2003, 2004a, 2005b, c) attack the philosophy of property possession by pointing out that there are problems with the linking up of a property to a particular. Some have suggested to me that my arguments only work if locality is assumed, and a non-local property possession could involve a particular possesses a property not by directly connecting to the property, but by being involved in $\mathrm{AD}$ with it. The arguments against $\mathrm{AD}$ given in this section would specifically attack there being any such AD property-particular linking.
} 
or contacting.) On the one hand, it is not uncommon to see philosophers discussing serious problems to do with concept of touching, contacting, and/or attaching and abutting between any two physical items ${ }^{159}$ (examples will be given below). Some argue that there could be reasons to think it is impossible. ${ }^{160}$ But on the other hand, many physicists and philosophers nevertheless seem to discuss particles as if they collide, as if there is particle interaction going on in nature via touching, contacting and colliding in the way that macroscopic DIV2 objects touch and contact. Often a physicist or philosophers will assert in an article or book on one page that quantum abstract atoms cannot contact or touch, and then a few pages later they will tell us that they can. To get a taste of this dual-natured discussion, consider just one of many examples that could be given, found in van Inwagen (1990). First, on page 34, he maintains in a parenthetical remark that "It is in fact probably meaningless to say of two electrons that they are in contact." But then in a passage on page 19 he appears to tell us that a prominent feature of subatomic particles (which electrons can only be included) is that they can be in collisions with one another (I assume that collision here means something along the lines of contact to the average DIV2oriented reader of van Inwagen's book), and where it appears to go against the case given later in his book where, at least for electrons, we are told that they cannot contact:

...those things like elementary particles which are not clear cases of material objects but which share many of their salient features (they are substances or continuants; they can move about and collide with and rebound from one another; they have masses). ${ }^{161}$

This is common among philosophers and physicists: they discuss particle collision and/or contact, but also often inform us that it doesn't occur, often on the very same page or in the very same paragraph. Often philosophers or physicists do not even mean to denote a touching and/or contacting event, but nevertheless use the words "touching" or "contacting", for example, to get their point across and describe what is going on between particles. Consider a passage from Lange:

We found it difficult to understand how two bodies could touch: being material, they cannot share a point, but if they do not occupy the same place at the same time, then they must be separated by at lest one point, and so there is a gap between them even while they are supposedly in contact. Fields can rescue locality from this difficulty. Suppose each particle of matter surrounded by a very short-range field of highly repulsive force... The field surrounding a given particle then causes an immense repulsive force on any particle that comes very near to the given particle. When two particles "collide," they do not touch, but they come near enough for each to feel a tremendous repulsive force from the other's field. So the two particles appear to bounce off each other. Each interacts with the field at that moment at its own location, so locality is upheld. Although one body excludes any other from the locations it occupies, those locations can be occupied simultaneously by a field and matter. ${ }^{162}$

\footnotetext{
159 See Lange chapter 1.

160 Kline and Matheson (1987).

161 Van Inwagen $(1990,19)$.

162 Lange (2002, 35).
} 
Lange refers to colliding particles that do not touch. Presumably, he is using the word "collide" to keep his words from becoming mysterious and hard to grasp. (This is likely what van Inwagen is doing also, and probably is also the case with the countless others I could cite who also do this.) But this passage provides a good example of how philosophers and physicists will often use the talk of two particles that "collide," "touch," "contact," when they don't actually mean that any of these are really happening between or among particles.

The evidence of this article shows that particles cannot involve any of these, nor any causal connection between one atom state to another. To understand what I mean by "atom state," consider the diagram below that illustrates what is observed regarding quantum abstract atoms. According to the diagram, since quantum abstract atoms $\mathrm{A}_{1}$ and $A_{2}$ at present moment $p_{1}$ cease to exist, whereby the quantum abstract atom at present moment $\mathrm{p}_{2}$ comes into existence, there can only be a breakage between any alleged continuity of the existence of atoms from $\mathrm{p}_{1}$ to $\mathrm{p}_{2}$, whereby what comes into existence at $\mathrm{p}_{2}$ is in some sense "new" atom-stuff (quantum abstract atomistic energy). It is "new" not in the sense that there has never been a quantum abstract atom that is indistinguishable from atom $\mathrm{A}_{3}$ at present moment $\mathrm{p}_{2}$ at any earlier present moment (i.e. at any moment "before" $p_{2}$ which no longer exists). ${ }^{163}$ Rather, it is new in the sense that it is an abstract atomic instance that does not have any causal connection to the past moment $\left(\mathrm{p}_{1}\right)$ and no causal connection between atoms at $\mathrm{p}_{1}$ and atoms at $\mathrm{p}_{2}$, and thus it "new" since it is completely existent in the present, not causally connected to any past moment. This is in accord with quantum empirical evidence (i.e. quantum randomness), and with philosophical reasoning I have given elsewhere about the nonexistence of time and continuous (unbroken) change from one moment to the next. What I have just written is in accord with the over two thousand year old philosophical discussion of the "problem" of change: if an item changes it can only cease to exist where a new items comes into existence. ${ }^{164}$ The reason $\mathrm{A}_{3}$ is new is the same reason that there is a breakage between moments $\mathrm{p}_{1}$ and $\mathrm{p}_{2}$ : because there cannot be any temporal relations between moments $\mathrm{p}_{1}$ and $\mathrm{p}_{2}$, the two moments cannot causally interconnect, cannot have any sort of interplay with one another, and for that reason, they are not causally connected, but each is "new." The reason that there are no temporal relations between moments $\mathrm{p}_{1}$ and $\mathrm{p}_{2}$ is because there is no way for the relations to interconnect the moments and/or the atoms at those moments, for reasons discussed in 4.3 .

There are quantum abstract atoms $\mathrm{A}_{1}$ and $\mathrm{A}_{2}$ at the initial present moment, and then there is atom $A_{3}$ at another present moment, where the first moment is replaced by the second (when the first moment ceases to exist the second one exists). It is not as if $A_{1}$ and $A_{2}$, which are separate at $p_{1}$, become stuck together at $p_{2}$ like pieces of

\footnotetext{
${ }^{163}$ I put the word "before" in quotes because, on this quantum theory of time being discussed (which elsewhere I have named the R-theory of time, or replacement presentism, and which appears to be the only accurate theory of time for the mereological nihilist quantum reality since it is a theory of time that is based solely on the replacement of particles by one another) because it is the DIV2 way to refer to previous moments. Strictly speaking, according to R-theory presentism, there is no before and after the present moment, so all I mean by "before" is this: present moment that once existed but not no longer exists.

164 This is an endorsement of the philosophy of presentism, and perhaps the specific variety of presentism I discussed in Grupp (2005a), which contains arguments for the Buddhist atomistic philosophy of time-which, in Grupp (2005a), I renamed the R-theory of time for specific reasons outlined in Grupp (2005a).
} 


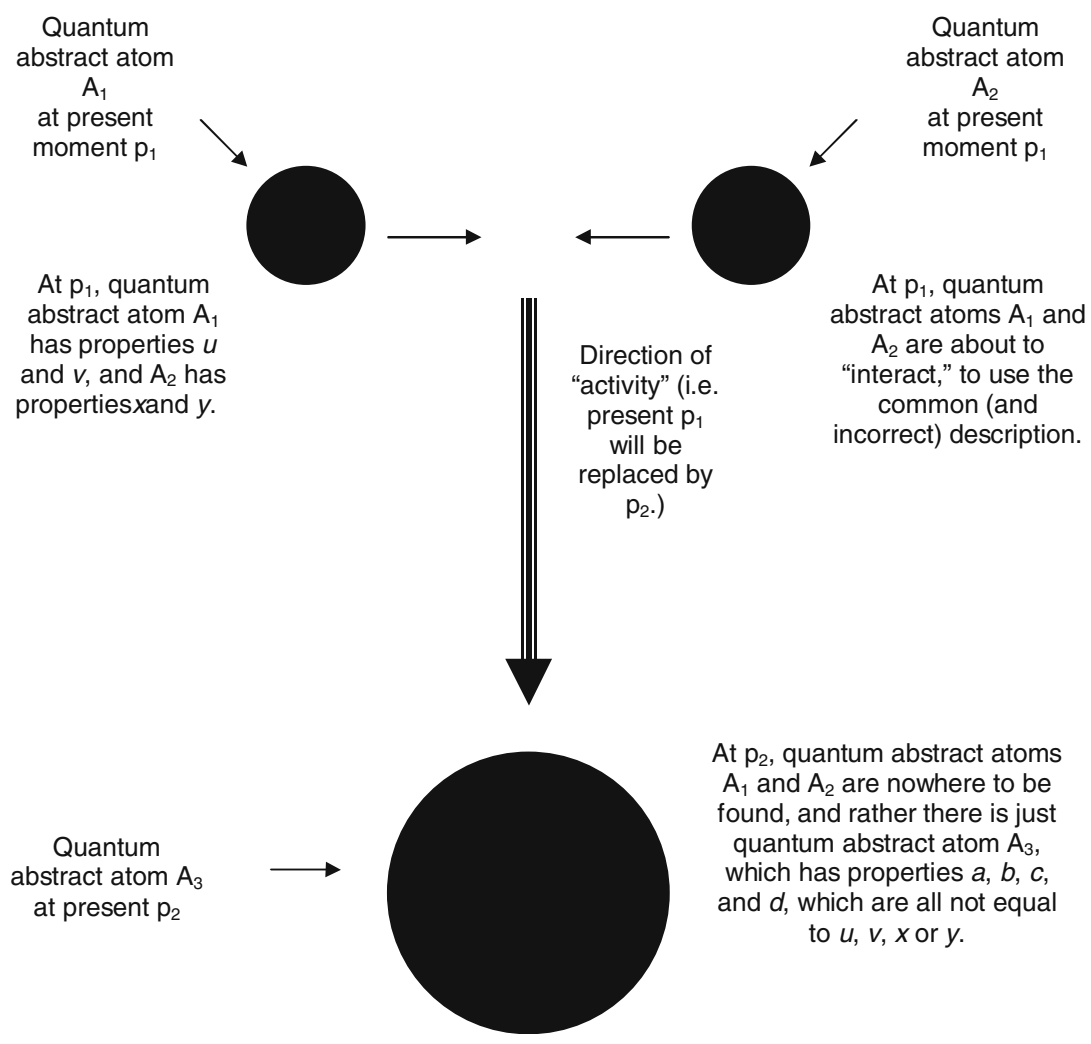

putty, where at $\mathrm{p}_{2}, \mathrm{~A}_{3}=\mathrm{A}_{1}+\mathrm{A}_{2}$. This is likely the way the DIV2-oriented, classical concept oriented mind will fall into believing what is going on from $\mathrm{p}_{1}$ to $\mathrm{p}_{2}$. But noting about any philosophical or scientific evidence that could be presented supports this, whereas the thesis of "newness" just introduced above, where $\mathrm{A}_{3}$ unconnected to a past time, and thus is new at $\mathrm{p}_{2}$, is supported by the evidence. "Between" $\mathrm{p}_{1}$ and $\mathrm{p}_{2}$ the quantum abstract atoms $\mathrm{A}_{1}$ and $\mathrm{A}_{2}$ do not contact, touch or interact in any sort of macroscopic manner, such as by bouncing off one another, or such as by surfaces interacting in some way. ${ }^{165}$ The diagram above may make it

\footnotetext{
165 Specifically why they do not contact or "touch" is not just a matter of empirical confirmation (confirmed in quantum reality). We can know through philosophical reasoning alone that if $\mathrm{A}_{1}$ and/or $A_{2}$ "touched", this would amount to past items $\left(A_{1}\right.$ and $\left.A_{2}\right)$ contacting a present (not-past) item $\left(A_{3}\right)$, and their interface would be past and not-past. I discussed at length in Grupp (2005a) why this is an impossible interface. This hints at a pervasive problem with causation in general in Western philosophy. In such philosophies of causation, moment $t_{1}$ is supposed to somehow contribute to the generation of moment $t_{2}$, or $t_{2}$ is supposed to somehow follow $t_{1}$ because of its interplay with $t_{1}$. If, however, $t_{1}$ is past and $t_{2}$ is present, then somehow the past and present must interact, and in one way or another-either via a temporal relation, or an abutment of moment, or something-something past and something present will have to directly attach which is impossible (see Grupp 2005a, section 2). This was noticed by some of the Buddhist philosophers from India, and it is one of many reasons in this article that an endorsement of presentism is taken up, where presentism involves the avoidance of causal relations, interactions, or processes across times, and instead a philosophy of the empirically verified non-causal quantum randomness is espoused.
} 
appear that what is really happening from $\mathrm{p}_{1}$ to $\mathrm{p}_{2}$ is that $\mathrm{A}_{1}$ and $\mathrm{A}_{2}$ are combining into one composite, $\mathrm{A}_{3}$. But this is not correct, for reasons I will give next. Although the diagram above shows $A_{1}, A_{2}$ and $A_{3}$ all to apparently involve size, in reality they do not, and they are shown here as if they do just for the sake of illustration; in fact each of these are to be considered point-sized quantum abstract atoms. Above we found that there cannot be any point-sized mereological wholes, and there cannot be any touching between point-sized items (or any items). Thus, each of the items in the diagram, actually being point-sized, can only be atomic. To understand the reasons why there is the aforementioned breakage between $\mathrm{p}_{1}$ and $\mathrm{p}_{2}$, imagine, contrary to what has been asserted above, that $A_{1}$ and $A_{2}$ do change into $A_{3}$. As the diagram above illustrates, the properties of the atom stuff that exist at $p_{2}\left(A_{1}\right.$ and $\left.A_{2}\right)$ are different properties than at $\mathrm{p}_{2}$. We can define the atoms in terms of their properties:

$$
\begin{gathered}
\mathrm{A}_{1}=[\mathrm{u}, \mathrm{v}], \\
\mathrm{A}_{2}=[\mathrm{x}, \mathrm{y}], \\
\mathrm{A}_{3}=[\mathrm{a}, \mathrm{b}, \mathrm{c}, \mathrm{d}] .{ }^{166}
\end{gathered}
$$

Notice that if we attempt to maintain that at $p_{2} A_{3}=A_{1}+A_{2}$, we can see this equation to be incorrect if we substitute values for $A_{3}, A_{1}$, and $A_{2}$ :

$$
\begin{aligned}
{[\mathrm{a}, \mathrm{b}, \mathrm{c}, \mathrm{d}] } & \neq[\mathrm{u}, \mathrm{v}]+[\mathrm{x}, \mathrm{y}], \text { or } \\
\mathrm{A}_{3} & \neq \mathrm{A}_{1}+\mathrm{A}_{2}
\end{aligned}
$$

Since $A_{3}$ is not equal to $A_{1}$ and/or $A_{2}$, then $A_{1}$ and $A_{2}$ must not exist at $p_{2}$, and must cease to exist when $p_{1}$ no longer exists, in order for $A_{3}$ to exist. In other words, if $A_{1}$ and $A_{2}$ change into $A_{3}, A_{1}$ and $A_{2}$ must cease to exist if they change from being what they are at $\mathrm{p}_{1}$.In addition to the evidence that there are no temporal connections from $\mathrm{p}_{1}$ to $\mathrm{p}_{2}$ given in 4.3 above and elsewhere, the discontinuity just described here-which is a discontinuity due to change (items change and thus cease to be themselves and thus can only cease to exist, or in other words, they pop out of existence) - from $\mathrm{p}_{1}$ to $\mathrm{p}_{2}$ indicates that contrary to what the DIV2 mind may want to believe, $\mathrm{A}_{1}$ and $\mathrm{A}_{2}$ are not sticking together at time $p_{2}$ to give rise to $A_{3}$. The DIV2-oriented mind may want to believe that $A_{1}$ and $A_{2}$ are really coming together to give rise to $A_{3}$ at $p_{2}$, much in the way in DIV2 one can bring together two distinct snowballs at one moment together a moment later where there seems to be no breakage between the moments, and where it appears that the snow at the first moment is the same snow that exists in the second. But for philosophical reasons just given, this is not what is happening in quantum abstract atomistic reality, and it is not what happens in what is com-

\footnotetext{
166 The metaphysical realist may want to maintain that our account here is incorrect, since the items here should not described only in terms of properties, but rather via properties and the property possessor, or perhaps in terms of a thin particular, and so on. In other words, the metaphysical realist will possibly find this account incomplete since there is no property possessor mentioned. But I don't mention it here because I found in Grupp 2005a (103-104) that such an account cannot coherently give an account of persistence through time. And in the case of a thin particular (bare particular) metaphysics. Also, in Grupp (2006a) I found that the other alternatives, the bundle account of nonbundle substances, and the so-called thick particular substance theory, are inadequate to describe persistence thorough change. Additionally, as pointed out in an above note, property possession is attacked by the reasoning of this article, even though I do not elaborate on this.
} 
monly referred to as "particle interaction." Rather, what is happening is that there are two atoms that exist at $\mathrm{p}_{1}$, and one atom at $\mathrm{p}_{2}$, and the non-connectedness between them prohibits causal interaction: $p_{2}$ is not generated by $p_{1} \cdot A_{3}$ is new: there appears to be amalgamation of particles, but it is most precise to maintain that $\mathrm{A}_{3}$ is distinct from $\mathrm{A}_{2}$ and $\mathrm{A}_{1} \cdot{ }^{167,168}$

Consider the case where an electron emits an photon. More precisely put, what happens is there is an initial present moment, $\mathrm{p}_{1}$, when electron $\mathrm{e}_{1}$ has an energy level $l_{1}$. And then shortly after there is a present moment $\mathrm{p}_{2}$ where a photon that has appeared to come from $\mathrm{e}_{1}$ 's direction and where at $\mathrm{p}_{2} \mathrm{e}_{1}$ no longer exists, and instead there is a new electron present, $\mathrm{e}_{2}$, with a new energy level, $\mathrm{l}_{2}$. There is discontinuity between $\mathrm{p}_{1}$ and $\mathrm{p}_{2}$, so it is not the case that $\mathrm{e}_{1}$ was "cut" apart into pieces, as if a piece of electron was ejected or cut off. Rather, it is the case that $\mathrm{e}_{1}$ existed, then ceased to exist whereupon $\mathrm{e}_{2}$ and the photon began to exist. So, on this example, there are three atomic items to consider: (1) $\mathrm{e}_{1}$ at $\mathrm{p}_{1},(2) \mathrm{e}_{2}$ at $\mathrm{p}_{2}$, and (3) the photon at $\mathrm{p}_{2}$. This appears like a splitting of $\mathrm{e}_{1}$. This scenario leads the DIV2 human observer - that might be tempted into believing in temporal parts, or identity through time-to believe that $\mathrm{e}_{1}$ becomes photon and $\mathrm{e}_{2}$. But points cannot have parts, and thus point object $\mathrm{e}_{1}$ and $\mathrm{p}_{1}$, which is one item, cannot become two, electron $\mathrm{e}_{2}$ and the photon at $\mathrm{p}_{2}$. Rather, $\mathrm{e}_{1}$ changes cannot be a composite. Rather, $\mathrm{e}_{1}$ changes (into $\mathrm{e}_{2}$ and the photon), and the only way this can occur for an atomic entity that does not have parts that can mix is by ceasing to exist entirely so that new energy manifestations can exist.

\footnotetext{
167 In response to the information in this section, my students often ask me: "But don't particles collide and smash apart in atom-smashers, sending pieces of the particles splattering all over? How is that not a collision?" Here is a response to this question:
}

Why do physicists want to smash atoms? The simple answer-to find out what is inside them —-has an element of truth, but there is a more general reason. Atoms-smashing is really a misnomer...

It is important to understand that these many different unstable particles are not in any straightforward sense the constituents of protons, neutrons, or electrons. When electrons and positrons collide at high energy they do not 'break open and spill out a shower of subatomic debris. Even highspeed collisions involving protons, which certainly do have objects (the quarks) inside them, do not actually involve the protons being smashed apart in the usual sense. It is better to envisage the debris which emerges from these collisions as being created 'on site' out of the energy of impact. (Davies 1984, 80-81)

This part of Davies 1984 also provides a good example of the confusing way that physicists talk about their empirical findings, since on page 80 Davies describes the situation initially as particle collision. He corrects himself, but often physicists will not do so. But then notice in the second to last sentence of this paragraph, Davies refers to what is going on as "high-speed collisions," but then immediately after he maintains that the collisions "do not involve the protons being smashed apart in the usual sense." This gives the reader confusion. Since the particles observed before the new energy do not equal the particles observed after, the particles after can only be new particles. Thus, it perhaps would have been better to merely state that "particles appear to move toward one another, and at the point where they should be imagined to coincide, they instead go out of existence and a plethora of new particles come into existence."

${ }^{168}$ If reality is propertyless at the quantum level, as discussed in footnotes before this one, this account of the breakage with the replacement of $\mathrm{p}_{1}$ by $\mathrm{p}_{2}$ would be misleading since it is given in terms of properties. But I only say it would be misleading, not wrong (and thus the property alteration description just given is worthwhile in that it illustrates the needed conclusion), because a propertyless reality actually also likely leads to the very same conclusion, as I showed in Grupp 2005a (Sect. 5.1). 
In what has been given in this subsection to this point, we can see why there is only popping in and out of existence, not amalgamation or contacting. But physicists often use the word "amalgamation" to describe the account of quantum abstract atom alterations I have just described. Consider the passage from the physicist, Gordon Kane, on these issues:

Nearly all particles are unstable and decay into others. The word decay has a technical meaning in physics-one particle disappears, typically turning into two or three others. A major difference between the way decay is used in physics and its use in everyday life or biology is that the particles that characterize the final state are not in any sense already in the decaying particle. The initial particle really disappears, and the final particles appear. The photons that make photons that make up the light we see provide an example: The photons emitted from a light bulb when it is turned on are not particles that were in the bulb just waiting to come out, and photons that enter our eyes... are absorbed by the molecules in our eyes and disappear. All particles can be created or absorbed in interactions with other particles. ${ }^{169}$

What is thought of in quantum reality as by physicists and philosophers as interaction, particle collision, or quantum abstract atom interaction, is really a very misleading way of describing quantum reality. These descriptions should not be used, and instead the phrases "particle replacement," or "quantum abstract atom vanishing and appearing" should be used instead. The words "interaction," "touching" or "contacting" regarding quantum reality are words that appear to actually mean momentariness (short-lived). I suspect that quantum physicists of the past began using the erroneous descriptions in order to discuss their observations in a way that makes sense to the DIV2-oriented empirical mind, but in fact it just leads to significant error and misinformation.

\subsection{Zeno's Measure Paradox is not a paradox}

So far I have shown that there are no relations between atoms, and atoms are pointsized. This leads to the Measure Paradox (or Zeno's Measure Paradox): an extended continuum is an aggregate of unextended points. This "paradox" is also called the Geometric Paradox, ${ }^{170}$ or Zeno's Paradox of Plurality. ${ }^{171}$. But according to the reasoning of this article, it is no paradox at all, since unextended points (or atoms) cannot give rise to a magnitude. In other words, point-sized philosophical atoms cannot compose empirical reality.

For over two thousand years, many philosophers have had reservations about how a line, or a geometric entity of one or more dimension, can be extended if it is an aggregate of unextended parts. But in recent decades, philosophers often appear at ease with the Paradox, due to the work of Adolf Grünbaum, in his alleged solution of the Paradox, and his discussion of the interconnectedness (called the set-theoretic union), of the points in a manifold. Before Grünbaum, the Paradox often evoked the question: If the basic constituents of an extended line, plane, volume are infinitely

\footnotetext{
${ }^{169}$ Kane $(2000,19)$

170 This is what Grünbaum calls it $(1955,165)$.

171 Zimmerman (1996a, 1).

型 Springer
} 
many unextended points, then how can the aggregate ${ }^{172}$ of the unextended constituents not also be unextended? If the basic unextended parts give rise to an extended whole, does this not imply that $(0+0+0 \ldots) \geq 1$, since the unextended entities give rise to an extension?

Grünbaum's solution allegedly gets around this contradiction, but I will argue that it does not. There has been little debate in the literature about Grünbaum's alleged solution. When discussing the Measure Paradox, philosophers often merely inform their readers that Grünbaum has solved the paradox, and no further explanation follows. An example of this lack of discussion is found in Zimmerman's article, "Could Extended Objects Be Made Out of Simple Parts? An Argument for "Atomless Gunk,", where Grünbaum is merely mentioned at the beginning of the article as having solved the Paradox, but no further discussion ensues:
Although Zeno's mathematical paradoxes of plurality were long thought to raise insurmountable difficulties for the supposition that an extended thing could be composed of unextended simple parts, Adolf Grünbaum has shown that these paradoxes are significantly defused by Cantor's discovery of the distinction between denumerabily and non-denumerabily infinite numbers. If Grünbaum is right, the traditional reasons for doubting the consistency of "conceiving of an extended continuum as an aggregate of unextended ele- ments" have been laid to rest... ${ }^{173}$

Grünbaum's alleged solution fails however, due to the fact that, as Edgar writes: "Grünbaum proposes to solve the Measure Paradox by arguing that extended space must be conceived as a relation among uncountably many unextended elements." 174 Relations however are impossible, due to what was ascertained in 4.2 and 4.3. Therefore, I do not see how Grünbaum's work is to provide a solution to the Measure Paradox. Rather, it seems the Measure Paradox is not a paradox at all, and merely reveals what quantum mereological nihilist reality involves: there are only quantum abstract atoms, and they cannot give rise to magnitudes.

\section{The DIV2 conceptual (non-existent) items}

In the next two sections I do three things: discuss the DIV2 conceptual reality that does not exist (Sects. 5.1-5.3), and that discussion leads to discussion of two quantum issues: quantum atomism (Sect. 6) and what I call the philosophy of immaterialism (5.4). I start by showing that space and time do not exist (Sects. 5.1 and 5.2). The conclusions I draw here in Sect. 5 will be used to show in Sects. 6.2 and 6.3 that there are no quantum paradoxes.

\subsection{Space does not exist}

Space and time are so ingrained in the DIV2 mind that it is very relevant to merely point out the metaphysicality involved with space and time, in addition to showing

\footnotetext{
${ }^{172}$ Grünbaum tells us that the Zenoic Paradox arises from the Zenoic manner of considering the points adding up, aggregating, or summing up to a magnitude.

173 Zimmerman (1996a, b), 1.

174 Edgar (1979, 324).
} 
that they do not exist. Space and time are considered to have parts by physicists and philosophers (the parts of time are called temporal parts in the case of time, and in the case of space the parts of space are called points in space, spatial locations, topological regions, and so on), and they are items that are used justify a plethora mereological and metaphysical theorization-including the so-called uncertainty principle in quantum mechanics, just to give one example. In the next two subsections, I will not only show that time and space do not exist, but also I will also give a general discussion of their aforementioned metaphysicality. Showing how space and time are non-empirical and extrasensory adds power to my arguments that show that they do not exist, and I need all the power I can get due to the degree of inculcation the typical human has that the invisible items, space and time, really in fact exist.

References to space and time are embedded in our common and widespread scientific and philosophical language. Yet if I merely ask the scientist or philosopher, Can you describe to me what space looks like?, I get little more than this sort of a response:

Space poses a central, intriguing and challenging question for metaphysics... On the one hand we are drawn to make very powerful statements about it. Everything that is real has some spatial position. Space is infinitely large, infinitely penetrable and infinitely divisible. On the other hand, despite our confidence in these strong claims, space seems elusive to the point of eeriness. It seems to be largely without properties, apart from the few strong ones just recited. It is imperceptible by any mode of perception. It has no material property, no causal one, it does nothing. It seems to have no feature which we can learn about by observation. Arguably, it has a prominent role in natural science but it is far from obvious just what it is. Though being spatial is a mark of the real, space itself seems, paradoxically perhaps, unreal[,] a mere nothing. ${ }^{175}$

If there is no answer to the question of what space looks like, then space is a metaphysical item. Space plays a "fundamental role in physics and philosophy,"176 but it is a theoretical construct of an invisible item that, according to the theoretical and metaphysical account of it, may not even interact with any other item.

There are two dominant theories of space. Einstein discussed each of these (notice in this passage also the way that Einstein tells us that the concept of space is a product of imagination):

(a) space as a positional quality of the world of material objects; $(b)$ space as container of all material objects. In case $(a)$, space without a material object is inconvincible. In case $(b)$, a material object can only be conceived as existing in space; space then appears as a reality which in a certain sense is superior to the material world. Both space concepts are free creations of the human imagination, means devised for easier comprehension of our sense experience. ${ }^{177}$

I will next show that there is no coherent account of space, wherein, our best account is that space does not exist. The argument I have to attack both the substantivalist and relationalist accounts of space attacks in one sweep: it shows that (b) the container model (the substantivalist account), involves fatal inconsistencies, and that

\footnotetext{
175 Nerlich $(1994,1)$.

176 Jammer (1993, ix).

177 In Jammer (1993, xv).

Springer
} 
(a) the relationalist model involves inconsistencies. The reason for this is because both of the substantivalist and relationalist accounts involve items that are interrelated. In the substantivalist account, the items that are interrelated are spatial points and/or spatial regions, and the relationalist account involves DIV2 matter items that are interconnected and interrelated, where according to relationalism, they give rise to a spatial network.

To move toward reductio, imagine that that either of the two accounts of space just given are correct. And imagine that there are two atoms, $\mathrm{A}_{1}$ and $\mathrm{A}_{2}$, which are either themselves interrelated, or the spatial locations they are located at are interrelated.

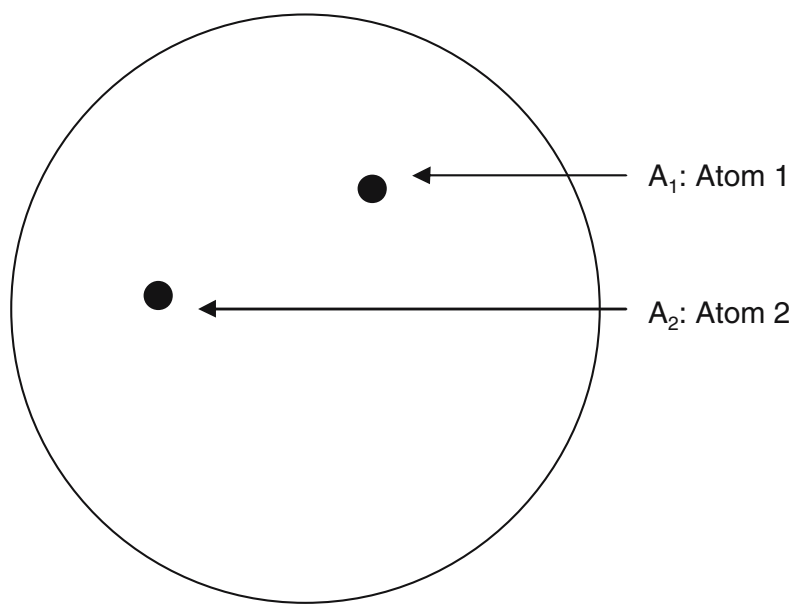

On the substantivalist account, $\mathrm{A}_{1}$ and $\mathrm{A}_{2}$ coincide with spatial points, call them $\mathrm{p}_{1}$ and $\mathrm{p}_{2}$, and $\mathrm{p}_{1}$ and $\mathrm{p}_{2}$ are allegedly interconnected by a topological relation. On the relationalist account, $\mathrm{A}_{1}$ and $\mathrm{A}_{2}$ are allegedly interrelated and by that interrelatedness it is presumed that there is spatiality. Physicists and philosophers very often consider the metaphysical relations between $A_{1}$ and $A_{2}$ and between $p_{1}$ and $p_{2}$ to be real items - as if they are real constituents of reality-and not mere tools for thinking about spatiality. Both accounts however fail, since the argumentation in Sect. 4 shows that there cannot be any such relations. But there are many fatal other problems that can be pointed out, and doing so it critical for the interests of the mereological nihilist, and thus I will describe them (even though the reasoning in 4.3 above would appear to be sufficient).

If there are complex relations (relations with parts), they would be mereological wholes, and thus could not exist due to the reasoning of Sect. 4, so we only have simple relations to consider. Partless relations either connect from $A_{1}$ and $A_{2}$ by "stretching" from $A_{1}$ to $A_{2}$ and all points between (analogous to the way a rope connects a boat and a dock), or they do not, and they are therefore only at $\mathrm{A}_{1}$ and $\mathrm{A}_{2}$, wherein they somehow interconnect nonlocality, as if to exhibit AD. Considering the first case, where the relation connects from where $A_{1}$ and $A_{2}$ are by a continuous (unbroken) simple relation that is at $A_{1}$ and $A_{2}$ and at any and every relation and location between $A_{1}$ and $A_{2}$ that it needs to be to give rise to the continuous (unbroken) connection. But we saw above in 4.2.3 that there cannot be 
any extended partless items. Distance or size is the non-coincidence of distinct items. (If they are not distinct, they are not at a distance from one another.) For there to be distance or size within the relation, it must involve inner structure so as to have inner parts that can be described as at a distance from one another. But that can only occur if relations have parts.

\subsection{Time does not exist}

Time is described as a set of moments that are interrelated. The relations are however susceptible to the attacks against relations given above, and thus do not exist. The A- and B-theories of time, so widely discussed by Western philosophers, but which depend on relations between non-identical times, are incorrect accounts of time, due to the reasoning of this article, and the non-existence interconnections between moments. ${ }^{178}$ Rather, what exists is only one indivisible present moment. For time to exist, there must be past and future moments each enmeshing with the present somehow to give rise to the idea of a series of moments. But without the interrelatedness of moments, and without moments being able to connect or touch in any way, there is no time flow, no time series: moments cannot be connected or interrelated. Moments cannot be positionally distinguished form one another, and if they cannot, they cannot be distinguished form one another at all, and there is thus only one moment: the present. For these reasons, the concept of time-a flow of time, or a series of time moments-is metaphysical and extrasensory, since it requires there to be non-experiential past and future moments that are linked by non-experiential temporal relations.

I use the word "extrasensory" to denote the impossibility of experience of a past or future moment: The DIV2 empirical mind only exists presently. If the DIV2 empirical consciousness could witness past times, where in doing so the states of DIV2 consciousness would exist as past in order to experience a past moment, then consciousness would be past and present, and thus could only consist of two distinct parts (the past part, and the present part-if consciousness is partless it could not have these opposing properties). Having two distinct parts, this distinctiveness requires that the part in the past, $\mathrm{C}_{\text {past }}$, cannot have continuity with the part of consciousness in the present, $\mathrm{C}_{\text {present }}$, due to the arguments against relations-such as relations between times-given above. ${ }^{179}$ Also, $\mathrm{C}_{\text {past }}$ and $\mathrm{C}_{\text {present }}$ cannot directly contact, since if they did, they would share a part via their overlapping, and that part would be both present and past, which is impossible. Without continuity, the consciousness at the present $\left(\mathrm{C}_{\text {present }}\right)$ cannot be the same consciousness as the one at the past $\left(\mathrm{C}_{\text {past }}\right)$. So the same consciousness could not experience both past and present moments, and present experience can only occur in the irreducible instantaneous present. But since real experience for human persons only occurs in the present and only exists presently (no human persons have had experiences of past moments, and thus believing there are such experience is merely metaphysical fabrication), then from what has been written above, real experience is only atemporal. Accordingly, DIV2 consciousness of time flow, and any feeling of time flowing that persons may believe they have, can only be an illusion, since consciousness is locked in the unextended irreducible now-which is to

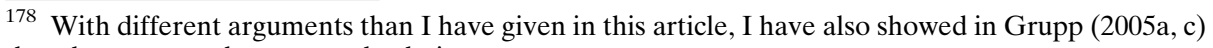
that there cannot be temporal relations.

179 And due to the much more detailed accounts in Grupp (2005a, c).

答 Springer
} 
say that experience of time, and the experience of time flow, is impossible, and can only be a presently generated hallucination.

In this illusory DIV2 generation of time-flow experience, somehow the present consciousness of DIV2 conceptualizers generates the belief that they witness surfaces and colors apparently that are moving, thus leading to the illusion of time flow-but where in fact each moment is new, and is unconnected to any other moment. When the DIV2 empirical experiencer somehow gains the ability, through present memory, to compare the present states of DIV2 surfaces and colors from present to present, the idea of movement comes about. Memory only occurs in the present, but it can apparently be about the past (about present moments that no longer exist), since we have illusory experiences of motion and time. The present can only be a durationless irreducible instant, and being durationless and irreducible, there is no movement within it.

With the experience of time, DIV2 observers are merely watching their own conscious states of surfaces and colors which appear to be moving about. Thus we are not measuring an item called time at all, but rather we are measuring our DIV2 consciousness of DIV2 surfaces and color rearranging in replacing presents, but where one never "witnesses" anything but his own conscious experience of arrangements of matter (surfaces and colors) rearranging; mind-independent times are never observed. When a human believes she is measuring time she is really just measuring her own conscious states of illusory DIV2 extended matter objects (objects with externally perceivable surface and/or color) as we witness them in DIV2 experiencing.

When one refers to temporal relations, or to spatial relations, she is referring to her experiences of empirical objects that are distinct and separate in our consciousness experiencing. When we here someone utter: "I see the lion here, and the zebra over there," the philosopher will typically assert that she is referring to spatial relations, and more specifically she is making a claim about the fact that the lion and the zebra are at a spatial distance form one another and are accordingly involved in spatial relations with one another. If I ask the philosopher, Can you describe what the spatial relations look like?, she will likely reply either that they are metaphysical and thus cannot be observed (and thus all that is observed is conceptual matter items with surfaces and/or colors), or that by merely seeing the lion and zebra at a distance from one another I am therefore seeing the relationship of distance in that instance of seeing the lion and zebra being not collocated with one another. But my DIV2 generation of matter-items experienced in consciousness only reveals surfaces and/or colors, and not the distance relationship - an invisible, metaphysical item, which reasoning above shows does not exist. With respect to this second account, since what is believed to be experienced externally in DIV2 consciousness are only surfaces and colors seen spread out from one another, then I do not see anything but a lion and a zebra, and there is no third mind-independent entity between then that I can label "distance." Instead of seeing distances between the lion and zebra, I only see other matter objects of the landscape (i.e., I only see other surfaces and colors): a few trees, a snake, and some bushes and dirt. Nowhere do I see a metaphysical relationship, and nowhere do I see anything but varying DIV2 color patches. The italicized word "between" in the statement, "... between the lion and zebra...," only refers to the DIV2 empirical surfaces and color patches (snake, trees, dirt, etc.). I do not see why asserting that there is a mind-independent, invisible, metaphysical relation called distance between lion and zebra could be a better way of describing 
what is between the lion and the zebra. And given the problems discussed above, and which I have discussed elsewhere, to do with mind-independent metaphysical relations which are believed to be real constituents of reality, it seems to remain coherent I cannot refer to them in my DIV2 experiencing. I do not see how I can avoid the conclusion that such an invisible relation is merely the DIV2 observer's way of passing over all the empirical objects between lion and zebra in order to quickly refer to the two of the color patches (zebra and lion) without having to arduously but precisely describe the situation as I just have. The concept of a metaphysical relation called distance between the lion and the zebra are the result of the quick ways that DIV2 experiencers refer to their reality, to the surfaces and colors. If we go to the Amazon, instead of asserting discussing every cell in every leaf and trunk of every tree, we will often just use one word, "forest" in order to save mental processing. Similarly, when I look at the zebra and the lion, it is more mentally economical to merely say they are 50 yards apart, rather than to refer to every empirical color patch between them. But this economical approximating can lead to the idea that there is something other than the mere color patches. We may imagine that there are real metaphysical items interconnecting items of reality, and this gives rise to the idea(s) that there is a thing called space.

In DIV2 consciousness, I do not witness a container, called space that the lion and the zebra exist within. I only experience the lion and the zebra (and the empirical objects that I perceive as being color patches assorted between the lion to the zebra). Space is typically defined as a continuum or manifold of spatial points interconnected by a topological metrical relation, and I certainly do not empirically witness that. Rather, I empirically observe objects lined up, where no relations or manifolds are perceived. This would imply that the word "space" is just a tool used to explain DIV2 surface and color patterning, but in fact we only experience the objects (the surfaces and colors), not the space or spatial relations, and we do not co-experience these assortments of color patches from moment to moment. In DIV2 conscious experiencing, all that is ever experienced are color patches of material surface, not mysterious items such as "locations" (temporal or spatial locations).

\subsection{A List of non-existents (if mereological nihilism is the correct theory)}

We have seen that space and time are impossible metaphysical items. The use of metaphysical relations are taken up in descriptions of DIV2 material empirical items, and physical and material reality involve such relations. Consider Lockwood's definition of "materialism:"

Materialism is the theory that everything that exists is material. I shall not attempt to define precisely what I mean by 'material'; but, roughly speaking, those things are material that occupy or take place in space, and whose existence is ultimately constituted by the properties and relations, actions and interactions of particles and fields, or whatever basic entities physics treats of. ${ }^{180}$

In addition to time, space, and matter, also causation is discussed in terms of metaphysical relations. A fundamental aspect of DIV2 is that academics have ubiquitously explained it in terms of metaphysical relations-but the relational

\footnotetext{
${ }^{180}$ Lockwood $(1989,20)$.

Springer
} 
reality only leads to a reality of paradox, antinomy, and philosophical problems, such as those I have discussed above (and elsewhere) and such as those discussed by other philosophers as far back as Parmenides.

I will next give a list of "commonsensical" and/or ordinary empirical DIV2 items that do not exist in a mereological nihilist reality, and which are largely metaphysical and invisible items, but which are widely assumed to exist by realist philosophers and surprisingly by many scientists.

(1) Space.

(2) Time and Time-flow.

(3) Motion. The non-existence of motion follows from the non-existence of time, point 2).

(4) Change. The non-existence of change follows from the non-existence of time and motion, points (2) and (3), and from the fact that the DIV2 experiencer only experiences an irreducible present moment, even though she will only believe she experiences change.

(5) Extension and Surface. I showed that surface and extension cannot exist in Sect. 4.

(6) Colors. The non-existence of colors follows from the non-existence of surface (point 5)) since to have color there must be extended surfaces. (Colors are not free-floating items.)

(7) Locations. This follows from 1) above: without space and time there are no locations in space and/or time.

(8) Causation. This follows from the non-existence of time and motion. Causes and effects are events that have some sort of interaction: one moments gives rise to, and/or comes before, the next. Causes and effects are events. Events are moments in time. So causation can be discussed in terms of time. With no time, there is no causation between non-identical moments. Cause and effect are just productions of imagination.

The impossibility of each of these are discussed at various places in this article. Only two of the DIV2 non-existents listed-namely surfaces and colors-are among those that DIV2 experiencers believe they perceive externally. In other words, only (5) and (6) are among the ordinary empirical experiences in DIV2 experiencing-all others are strictly metaphysical and unobservable items (why each is metaphysical is discussed below), that cannot be explained in philosophy without contradiction.

\subsection{Immaterialism and energy}

It is often assumed that the fundamental particles of reality are physical items. For example, Schaffer writes that "the physicalist claims that microphysical theory (or some future extension thereof) describes the fundamental level of reality on which all else supervenes." 181 I will argue at various places in this article, and specifically in this subsection, that there is no reason to believe that quantum atoms (electrons, gluons, etc.) are material items: the quantum abstract atoms are not describable

181 Schaffer $(2003,498)$. 
as being physical or material items, but rather only to describe them as being immaterial items.

Although I have not seen any philosophers put forth this theory, the idea that quantum particles are non-physical is an idea that is occasionally suggested. van Inwagen writes:

...submicroscopic objects like quarks and protons are ... not clear cases of material objects; nevertheless, every material object would seem pretty clearly to have quarks and protons as parts... A "part," therefore, need not be a thing that is clearly a material object. ${ }^{182}$

According to the inferences of this article, the only items that exist are quantum abstract atoms, and the following arguments show that they only can be considered to be immaterial items, and thus reality is immaterial:

1. Definition of "material" or "matter," comes from ordinary DIV2 empirical experiences, and consists of the DIV2 descriptions given above in 2.4.

2. Quantum particles (quantum abstract atoms) are utterly different sorts of objects than the material objects mentioned in 1: if a DIV2 item has property $P$, then a DIV1 item has property $\sim P$.

3. Conclusion 1: Quantum particles (quantum abstract atoms) are immaterial.

1. If immaterial quantum abstract atoms are reality, then reality is immaterial.

2. According to mereological nihilism, quantum abstract atoms are the only items that exist.

3. Conclusion 2: reality is immaterial.

Since the quantum abstract atoms are immaterial, then reality is immaterial. I call this position immaterialism (or mereological nihilist immaterialism). Immaterialism can be defined as follows: there is no material thing that exists; if an entity exists it is necessarily an immaterial item, and, more specifically, it is a structureless particle (quantum abstract atom). Given conclusions 1 and 2, the reasoning given in Sect. 2.4, and the case for mereological nihilism I present in this article, I am not sure how to avoid the conclusion that reality is describable by the conclusion of immaterialism (conclusion 2).

In premise 1 of the first argument, the word "material" in the previous sentence (and throughout this article) denotes the objects in reality that humans ordinarily believe exist in the world external to the DIV2 experiencer, and which are empirically perceivable items that (are believed to) exist in space (have a spatial location), have a spatial extension (i.e., have a surface and/or a color), and endure (or perdure) through a duration without flashing in and out of existence. van Inwagen writes:

A thing is a material object if it occupies space and endures through time and can move about in space (literally move about, unlike a shadow or a wave or a refection) and has a surface and has a mass and is made of creation stuff or stuffs. Or, at any rate, to the extent that one was reluctant to say of something that it had various of these features, to that extent one would be reluctant to describe it as a material object. Few philosophers would be perfectly happy

\footnotetext{
182 Van Inwagen $(1990,19)$.

Springer
} 
about calling a quark or a proton or even a large organic molecule; a material object, for one has to be very careful in ascribing any of the feature in the above list to such things; and talk about the surfaces of submicroscopic objects, or about the stuffs they are made of, tends to verge on nonsense... Some philosophers are happy to call clouds and forests and galaxies material objects and other are not. But virtually all philosophers believe that there are central, perfectly clear cases of things that fall under the concept material object. ${ }^{183}$

Mereological nihilism shows that items that (are believed to) have these material properties are the entities that do not exist since, as discussed above, if mereological nihilism is correct (which depends on the reasoning in Sect. 4), all DIV2 items are only mental concepts (not mind-independent items). Instead, we will find such properties to be impossible, and thus we will find that if any person or being (believes she/he has) experiences of a material world, filled with color patches and moving surfaces, then it can only be the case that they are hallucinating the experience of the materialism: materiality is the mental generation of concepts that do not map on to any mind-independent reality. The characteristics of matter discussed above and discussed in the van Inwagen passage are not characteristics of the particles of quantum physics. The quantum abstract atoms have been discovered to have no location, have no enduring existence or to involve any significant duration, they do not have structure, they do not touch, ${ }^{184}$ they exhibit little or no movement, they do not have spatial extension (they are not observed to have surface, and thus no color, and quantum particles cannot be directly empirically observed at all.), and compared to the "logic" ordinary empirical perspective, quantum reality appears non-logical. ${ }^{185}$ Most philosophers who discuss quantum objects typically erroneously claim they are not immaterial, but are the smallest physical objects.

I have been told since I was forming my earliest DIV2 memories at 2 years of age, that what reality is, consists of the set of surfaces and colors that I (believe I) experience outside of me. I have also been told that this DIV2 reality is material. But

\footnotetext{
183 Van Inwagen $(1990,17)$. In a note above I pointed out how often even metaphysical entities are sometimes also put into the definition of "matter."

184 Van Inwagen discusses this $(1990,34)$. Also, Hoffman and Rosenkrantz, in their book Substance, discuss this issue, and how it might lead to skepticism about the existence of parts and wholes (i.e., as evidence for mereological nihilism):
}

\begin{abstract}
Skepticism about the intelligibility of the relations which are commonly thought to unify or organize the parts of material objects... might be thought to provide... argument in favor of monadism [Hoffman and Rosenkrantz are using "monadism" here as synonymous with "mereological nihilism," see page 77 of their 1997]. Such skepticism or claims of ignorance might be fueled by developments in modern science, developments which are themselves well confirmed by experimental data. For example, the laws of physics imply that in all but extremely exceptional circumstances, no two physical objects touch (strictly speaking) because of the presence of repulsive forces between fundamental particles. (Hoffman and Rosenkrantz 1997, 78)
\end{abstract}

It is interesting to note the way quantum physicists also come to the conclusion that the fundamental particles (some of which, such as electrons or quarks, may be true philosophic atoms) apparently do not contact, touch or interact in any sort of macroscopic manner, such as by bouncing off one another, or such as by surfaces interacting in some way. Instead, what "interaction" in quantum physics means is really a misleading word that physics use, because "interaction" or "touching" or "contacting" of particles at the quantum level are words that appear to all be synonymous with the word "momentariness".

185 As discussed, DIV2 is in fact absurd, and DVIV1 is coherent. 
if I investigate and peer into it, if I probe these surfaces and colors, and if I try to find out what they are made of, I arrive at a quantum reality that only involves the aforementioned immateriality. In other words, if I study the objects of my ordinary empirical experience enough, I come to the discovery that these objects do not exist. The famous physicists, John Gribbin and Paul Davies, express a similar outlook in a passage about how investigation into matter leads the way out of materialism. This passage, which summarizes nearly all issues of quantum mereological nihilism, indicates that the immaterialist quantum abstract atoms are invisible energy, which will be relevant to the discussion near the end of this subsection:

It is fitting that physics-the science that gave rise to materialism-should also signal the demise of materialism... [T] he new physics has blown apart the central tenets of materialist doctrine... [Q]uantum theory... totally transformed our image of matter... [One of the primary sectors] of... quantum theory, known as quantum field theory... goes beyond even this; it paints a picture in which sold matter dissolves away, to be replaced by... excitations and vibrations of invisible field energy. In this theory, little distinction remains between material substance and apparently empty space, which itself seethes with ephemeral quantum activity. ${ }^{186}$

Due to the reasoning I presented up to this point in this article, I see now way to avoid immaterialism. But I do see philosophers (and physicists) attempting to veer around the thesis of immaterialism in order to claim that (somehow) quantum abstract atoms are material objects. If immaterialism is correct, the only way such a claim could be made, and that quantum objects could be considered to have the aforementioned material properties, is by plainly giving false description of quantum reality. This is in fact what I have found to be the case in the literature in philosophy and physics, where philosophers and physicists make the conclusion that quantum atoms are material by presenting obviously incorrect accounts of quantum objects in order to get to that claim. An example can found by merely looking at the same page of van Inwagen's widely discussed book that I have already cited in this section upon which van Inwagen claims that quantum atoms are "not clear cases of material objects," but where we can see van Inwagen non-inferentially resisting the immaterialist thesis in this passage:

There is one relation called 'parthood' whose field comprises material objects and those things like elementary particles which are not clear cases of material objects which share many of their salient features (they are substances or

$\overline{186}$ Davies and Gribbin (1992, 14).

Also on page 14 , in response to these ideas, and right after he passage just given, they write that: the culmination of these ideas [regarding quantum field theory] is the so-called superstring theory, which seeks to unite space, time, and matter, and to build all of them from the vibrations of submicroscopic loops of invisible string inhabiting a ten-dimensional imaginary universe.

As discussed in other notes of this article, string theory is a non-experimental, primarily mathematical theory of quantum reality. But what is interesting to note is that the theory agrees with virtually everything about the quantum mereological nihilist thesis of this article - except for one issue: the quantum abstract atoms are not points, but rather are vibrating strings or sheets of energy. This is even true on issues of non-contacting. From what I understand from physicists, these strings or sheets do not contact or touch, but only amalgamate or unify, as mereological nihilism involves. 
continuants; they can move about and collide with and rebound from one another; they have masses). ${ }^{187}$

I have shown in numerous passages in this article from the experimental accounts of physics that there is very little correct about this passage. I give textual evidence from physics that show that quantum objects do not collide, that they do not have any real persistence. So the mystery is: Why does van Inwagen, who is considered a leading American philosopher, present inaccurate information? It seems to me, as mentioned elsewhere in this article, that physicists could be partly to blame, as they often carelessly describe their data, often in a way where they are merely trying to carry out the nearly impossible task of explaining DIV1 logic understandable to the DIV2 consciousness, thus giving rise to mistakes by those who interpret it. ${ }^{188}$

The philosophy of immaterialism appears to be closely linked to the concept of energy, and to the idea that quantum abstract atoms are fleeting points of energy. For example, the photon was discovered by Max Planck to apparently be composed of energy and describable by the equation $E=h f$ ( $f$ is frequency, and $h$ is Planck's constant). (Interestingly, it was perhaps the discovery of this equation, and the discontinuous "motion" is predicted, that more than any other equation brought out what is called the new physics, which is the quantum-oriented physics.) Then it was found that all particles could be fully described in terms of their energy, or, in other words, as being energy (what Ford calls "the energy of being" in a passage below). Heisenberg wrote: "[e]nergy is in fact the substance from which all elementary particles, all atoms and therefore all things are made." ${ }^{, 189}$ Also, the very popular new theory of quantum physics called string theory (discussed above) also involves the idea that all basic building blocks are composed of strings of energy.

The reason that the quantum particle energy thesis, and the philosophy of immaterialism, are closely associated-even to the point that we can maintain that quantum abstract atoms are immaterial energy - is due to the following argument:

Quantum abstract atoms are immaterial,

Quantum abstract atoms are energy.

Conclusion 3: Quantum abstract atoms are immaterial energy.

The thesis that quantum abstract atoms are immaterial points of energy might seem to be a similarity between DIV1 and DIV2, contrary to the idea that they are the inverse properties of one another, since they are both are ultimately energy (DIV2 objects are energy due to Einstein's $E=m c^{2}$, which shows that all massy items are energy). This would contradict the principle that if a DIV1 object is P then a DIV2 object is $\sim \mathrm{P}$. This however assumes that there is a macroscopic reality "out there" to be called $m c^{2}$ — which the mereological nihilist, and the quantum physicist, have found there is not. At any rate, the essence of being a DIV2 conceptualizer of surface and color experiences is to not see the world as energy, but as stagnant matter, which appears very different than energy, as is seen in the passages above, and the one I am about to give from Professor Ford. The immaterial energy that is

\footnotetext{
187 Van Inwagen $(1990,19)$.

188 Interestingly, physicists even admit that their usage of terminology is imprecise, admitting it is highly metaphorical. See Kane (2000) (39) where he discusses this (as if it is a good thing), maintaining that "physicists have chosen to humanize [the equations and data]..."

189 Heisenberg (1999 (1958)), 63.
} 
the quantum abstract atoms is not quite the same as the potential and kinetic descriptions of energy given in Einstein's relativity-due to the fact that the "behaviors" of quantum objects are different than those of macroscopic objects. A passage from the physicist, Kenneth Ford, describes what the word "energy" means when we are considering quantum energy:

[T]he total importance of energy springs not just from its variety of form, but from its conservation: the total amount of energy in the cosmos remains always the same, since the loss of one kind of energy is always being compensated by the gain of another kind of energy... In the particle world, there are just two significant forms of energy: kinetic energy and mass energy. Kinetic energy is energy of motion; mass energy is energy of being. ${ }^{190}$

Ford's passage emphasizes how the kinetic energy versus potential energy distinction, being more of a metaphysical DIV2 conception, can be given in terms of motion and existence, as if a quantum abstract atom is an irreducible bit of existence.

\section{The mereological nihilist interpretation of quantum mechanics: no space, no metaphysics, quantum atomism, and the metaphysically generated illusion of quantum paradoxes}

In this section, I show how mereological nihilism is an alternative interpretation to the interpretations of quantum mechanics we have been given (Bohmian, Multiple Worlds, Copenhagen, etc.). What is most important about the mereological nihilist interpretation of quantum physics is that there are no quantum paradoxes, which makes the mereological nihilist interpretation diametrically opposed to the other interpretations. Physicists have fooled the world into believing that there are paradoxes of reality at the quantum level, and by the word "paradoxes," we are asked to believe that reality involves absurdities and impossibilities. Deriving these paradoxes is strictly a metaphysical endeavor which has nothing to do with the I-empirical data. It is one of the great threats to critical thinking in the contemporary world, contributing to widespread abandonment of critical thinking by people world-wide. These metaphysical paradoxes have led many people to, in some sense, forget that it is only through strict non-paradoxical and straightforward statistical mathematics that led to the quantum revolution (by Heisenberg, Dirac, etc.) and the discovery the quantum nature of ultimate reality. The creation of quantum physics is a stunning, but straightforward, scientific undertaking, based soundly in standard scientific inductive analysis. To assert that there are metaphysical absurdities and paradoxes at the end of this strictly logical, empirical, and mathematical pursuit, and to claim a logical mathematical analysis brought about this illogical reality, seems to go against the entire spirit of science. Scientists are famous for relishing, and delving into, mysteries. Outside of quantum physics, I do not know of one other scientific area that involves the assertion that the mysteries that the scientific area turns up are not to be investigated further, and rather, they are merely to be accepted, and no further investigation is needed. So why does quantum physics involve the assertion that there are facts about reality that do not need questioning and examination, and where we are asked to believe that they are merely absurdities that reality involves, period? This would be

\footnotetext{
190 Ford (2004), 18. 
analogous to astrophysicists maintaining that since dark matter appears to be a paradoxical mystery, inquiry into what dark matter is, is not needed, and no further investigation is needed, we are to just accept that it is out there without inquiring. It would be analogous to if physicists in the late 1887 had maintained that since the ether seemed paradoxical due to the findings of the Michelson and Morley experiment, we should not question the ether, and just accept its existence, and accept that it is a paradoxical entity. And this would be like demanding that research at Princeton on telepathy ${ }^{191}$ be stopped, since we should just accept that telepathy is a fact, and no explanation is needed. Since the cutting edge areas of science are replete with mysteries, if all scientists had the attitude that so many quantum physicists have exhibited, then much of the progress of science would instantly halt.

Imagine if the dark matter astrophysicists announced that their theory of galactic rotation is an utter paradox and mystery, since it involves the idea that there are galaxies that have been scientifically verified to be both of mass $m_{1}$ and mass $m_{2}\left(m_{2}\right.$ is significantly less than $\mathrm{m}_{1}$ ), and since one object cannot have two masses in this way, nature therefore involves irreducible paradox. And then imagine that a few of the dark matter theorists openly, publicly and frequently berated almost anybody who happened to question them! Clearly this would be entirely opposed to the spirit of science, but it is exactly analogous to what some of the pioneering physicists (Feynman, etc.) have established.

The paradox-less mereological nihilist interpretation of quantum mechanics is similar to the waveless variety of the positivistic Copenhagen Interpretation, which is an interpretation that is closely associated with Bohr. But the paradox-less mereological nihilist interpretation of quantum mechanics involves (at least) one significant difference with the Copenhagen Interpretation: the reasoning above about mereological nihilism reveals that quantum reality is atomistic (unlike the aforementioned version of Copenhagen). There are specific reasons that Copenhagen does not arrive at this conclusion, but I will show that those reasons are to be rejected. The I-empirical findings of quantum reality are of partless objects and therefore of point objects (e.g. electrons are points; protons, which do not exist, are really just quarks and gluons, which are points). And quantum point particles are quantum abstract atoms, for reasons given above.

In this section, I will first give a general discussion of the aforementioned early version of the Copenhagen Interpretation, since pointing the problem with that version can help to vindicate the mereological nihilist interpretation of quantum mechanics. I will also discuss the quantum paradoxes, showing that they are actually not paradoxes, and that they are the result of metaphysical concepts which can only be absurd due to the reasoning in Sects. 3 and 4 (among other reasons), and due to the empirical findings of the physicists themselves. Understanding that the paradoxes are merely misguided and incongruous metaphysics also leads directly to the mereological nihilist interpretation of quantum mechanics, as I will point out. Lastly in this section, I will further discuss relevant issues to do with the way that the metrological nihilist interpretation of quantum physics is an atomistic thesis, and where the avoidance of absurdist metaphysics and the illusion that there are paranormal quantum paradoxes leads directly to the nihilistic philosophy of quantum abstract atomism. Due to length limitations in this article, the only quantum

191 This is being carried out by the group known as "PEAR": Princeton Engineering Anomalies Group. 
paradoxes I will discuss are what seem to be the two most famous ones: waveparticle duality (Sect. 6.2) and the Heisenberg uncertainty principle (Sect. 6.3).

\subsection{The Copenhagen Interpretation}

In this subsection I do not give any sort of detailed analysis of the extremely popular (among physicists) Copenhagen Interpretation of quantum mechanics. My only intention is to discuss its most basic conceptual principles, and to show that those principles have some interesting similarities with mereological nihilism (which is perhaps not entirely surprising, since both theories are non-metaphysical and quantum-based. ${ }^{192}$ ) Discussing the Copenhagen Interpretation will lead to a discussion on wave-particle duality and other alleged quantum paradoxes, where I will find that there are no such paradoxes. The Copenhagen Interpretation is the interpretation of quantum mechanics accepted by physicists. ${ }^{193}$ It has many varieties, where one variety often has opposite features of another (e.g., some maintain that there are waves, some don't). The version of the Copenhagen Interpretation I will be discussing is an early anti-metaphysical, ${ }^{194}$ even positivistic (or phenomenalist) interpretation of quantum physics. The Copenhagen Interpretation comes from the great inventors of quantum physics: "Bohr, Heisenberg, and Pauli laid the foundations for interpretation long ago, and nothing that has since been discovered really undermines that groundwork." ${ }^{195}$ It is an attempt to make sense of quantum reality to the DIV2 experiencer (who is partial to DIV2 modes of experiencing): to the ordinary empirical perspective, quantum reality appears to be a domain of intangibleness which allegedly generates a "crisis of concepts" generated by it. ${ }^{196}$

At the start of Faye (2002) we find this passage: "the Copenhagen Interpretation is mostly regarded as synonymous with indeterminism, Bohr's correspondence principle, Born's statistical interpretation of the wave function, and Bohr's complementarity interpretation of certain atomic phenomena." We will find that each point raised here is very similar to the philosophy of mereological nihilism being purported in this article. Other interesting similarities between Copenhagen and mereological nihilism are as follows. Both theories involve a strict rejection of determinism, and involve serious questioning of causation in general, since quantum reality does not involve patterns that can be labeled as causal or regular. While

\footnotetext{
192 Being more precise, the Copenhagen Interpretation was non-metaphysical and positivistic at its Bohrian outset, but this changed when varieties of the Copenhagen Interpretation arose. In this section, I consider only the original Bohrian formulation of the Interpretation to be in line with mereological nihilism.

193 Griffiths (1995, 3-4), Stenger (2000, 213).

${ }^{194}$ It is very odd that the Copenhagen Interpretation gets lumped together with metaphysics. This is not so for the Bohrian interpretation. Stenger writes that: "Bohr...tended to adopt the... extreme positivist doctrine that an unmeasured property is too meaningless, too metaphysical, to even talk about." (Stenger 2000, 117) The Copenhagen Interpretation is used by non-philosophers often to attempt to establish a mystical metaphysics, but this is to completely sidestep what Bohr intended, which was to remain more-or-less positivistic, which is to say: to remain as anti-metaphysical as possible. (Oddly, Stenger later implies that the positivistic Copenhagen Interpretation "raises deep metaphysical issues about what constitutes reality," (132) and thus appears to commit the very error about applying metaphysics to Bohr's philosophy, and contradicts the passage on 117.)

195 Omnés (1999a, 3).

196 Omnés (1999a, 3).
} 
Copenhagen involves the idea that there is an indeterministic quantum reality, ${ }^{197}$ for reasons given above, it is safe to say that this indeterminism is such that it leads to an obliteration of causation, just as is the case with mereological nihilism.

Now for the differences that the Copenhagen Interpretation has with mereological nihilism. Surprisingly, the very first versions of the Copenhagen Interpretation denied that a quantum reality actually exists, and rather involved the idea that only consciousness exists. ${ }^{198}$ Herbert describes this as the position that " $[\mathrm{t}]$ here is no deep reality... There is no quantum world. There is only abstract quantum description."199 Unless consciousness is identical to the quantum abstract atoms (which apparently, according to the Copenhagen Interpretation, it is not), this is a major difference between the Copenhagen Interpretation and the quantum-based mereological nihilism. This is the primary difference I know of where there appears to be a conflict between the claims of mereological nihilism and that of the Copenhagen Interpretation. But in fact it may not be much of a conflict: I am not convinced that postBohr physicists really hold to this aspect of the Copenhagen Interpretation on a regular basis. In other words, physicists hold to the tents of the Copenhagen interpretation, except for this one (and they will still consider themselves Copenhagen since they meet the rest of the tenets of Copenhagen). ${ }^{200}$ If one listens to the way contemporary quantum physicists talk, they maintain that there is a quantum world, and (as seen in one of the Griffiths' passages just given) when we are not looking it is wavy, and when we look at it those waves collapse into I-empirical points. Therefore, there is something there when we are not looking, and Copenhagen has changed since the original Bohr idea that there is no quantum world. Herbert refers to this as a principle tenet of the original formulation of the Copenhagen Interpretation, but we can find countless instances of physicists who tell us they are part of the Copenhagen Interpretation tradition, telling us that there is a quantum reality. In fact, Herbert also refers to the quantum world as being real; to give one example, consider the wording of this passage: "...new quantum facts force physicists to admit that the world most certainly rests on some bizarre deep reality." 201 For these reasons, I find that we can reject the idea that there is no quantum reality as being part of the current usage of the Copenhagen Interpretation by physicists.

From this we can see how the Copenhagen Interpretation promotes the idea that consciousness creates reality. ${ }^{202}$ I take this to mean-in mereological nihilist terms - that there is no DIV2 reality without consciousness creating it. ${ }^{203}$ This is in precise agreement with mereological nihilism, at least from the perspective that both theories involve the strict position that consciousness creates DIV2, and there is no DIV2 when nobody is looking (generating experience).

\footnotetext{
197 Faye (2002).

198 See Herbert $(1985,16-18)$.

199 Herbert (1985, 16-17).

${ }^{200}$ Gibbins writes that "there is no one Copenhagen Interpretation of quantum mechanics... [O]ne should not think of the Copenhagen Interpretation as a single consistent Interpretation of the theory." $(1987,47)$

${ }^{201}$ Herbert $(1985,55)$.

202 Herbert $(1985,17)$.

${ }^{203}$ John Wheeler wrote: "No elementary phenomenon is real phenomenon until it is an observed phenomenon. (Quoted in Herbert 1985, 18)
} 
The rest of this section deals with quantum paradoxes and quantum atomism, in order to lead into the next subsection. Both of these involve the wave half of the wave-particle duality thesis, and with the discovery that such waves are entirely metaphysical and can be shown to not exist. The positivistic accounts of the Copenhagen Interpretation do not accept the wave function as real, ${ }^{204}$ but, as the Griffiths' passages show, physicists however often put aside this aspect of Copenhagen in order to metaphysically theorize that, for example, "according to quantum mechanics, every probability wave extends throughout all of space, throughout the entire universe." 205 This is to maintain that, although particles appear to move in wavy patterns, the wave itself is never observed, and only particles are observed. In other words, the wave is entirely metaphysical, and the particles (which are points) are I-empirical. Usually physicists refer to this Iempirical experience of the particle as measurement, and refer to the wave as what is there when nobody is looking, and the wave undergoes a transformation from wave-status to particle status when measurement is carried out (i.e., when an observer attempts to view the particle). Griffiths, who wrote the primary textbook that is used for upper level quantum mechanics classes, discusses this early in this book in a section on the Copenhagen interpretation, and where he uses " $\mathrm{C}$ " to denote measurement (experience of a particle) and " $\psi$ " to denote the metaphysical wave (non-experience of the particle):

It was the act of measurements that forced the particle to "take a stand" (though how and why it decided on the point $\mathrm{C}$ we dare not ask). Jordan said it most starkly: "Observations not only disturb what is to be measured, they produce it... We compel (the particle) to assume a definite position." 206 This view [is]... the so-called Copenhagen Interpretation... [that is] associated with Bohr and his followers. $^{207}$

Evidently... measurement radically alters the wave function... We say that the wave function collapses upon measurement, to a spike at the point $\mathrm{C}$ ( $\psi$ soon spreads out again...). There are, then, two entirely distinct kinds of physical processes: "ordinary" ones, in which the wave function evolves in a leisurely fashion... and "measurements", in which $\psi$ suddenly and discontinuously collapses. $^{208}$

204 Stenger $(2000,132)$.

${ }^{205}$ Greene $(2004,90)$. Greene does not entertain the idea that the wave is a mere mathematical descriptive tool, and not an outcome of mere particle observations, even though, as I will cite, below on this same page he refers to this as "[mere] description." It is mysterious that the interference patterns of the two-slit experiment arise, as if the particles interact across time. But just because it is a mystery does not mean it leads to a contradiction in quantum mechanics, but rather a limitation due to DIV2 experiential shortcomings.

${ }^{206}$ Mermin, David N. "Is the Moon There When Nobody Looks?" Physics Today. April 1985. Page 38.

207 Griffiths $(1995,3)$. Notice how Griffiths' refers to the quantum world as if it is waiting there when we are not measuring it, waiting to be measured. This will be significant at the end of this subsection when we discuss how Bohr originally thought there is no quantum world, only abstract measurement. ${ }^{208}$ Griffiths (1995, 4-5). Notice how Griffiths' uses the term "physical" to describe these particle processes, which shows how physicists assume the position that particles are physical, in disagreement with the findings above regarding the philosophy of immaterialism. 
Waves are just waves, whether they exist in sound, light, or elsewhere. But waves, strictly speaking, do not exist. The water wave is merely $\mathrm{H}_{2} \mathrm{O}$ molecules moving up and down, but they do not do so in the same way, and thus the appearance of a moving wave exists. Notice how this diagram shows that the pieces of waves only move up and down:
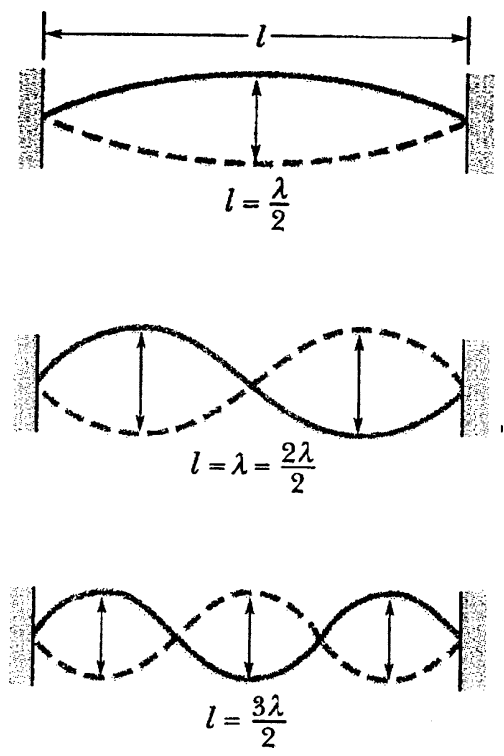

The same can be said for other waves in other mediums: waves are just unattached particles. If water waves do not exist, why would quantum waves exist? An important point is that the waves referred to above, being spatially extended items, cannot exist, given the reasoning of Sect. 4 above, and since the argumentation there showed that there can be no extended item, whether composite or non-composite. This is one of many reasons I will find that the wave part of the wave-particle duality equation does not exist: it is a metaphysical invention. Without waves, there is no wave particle duality.

6.2 No quantum paradoxes: the myth of wave-particle duality and uncertainty about location and motion

The argumentation in Sect. 4 shows that there can not be any extended objects. The waves in wave-particle duality are not points, they are (alleged to be) extended items. Therefore the reasoning in Sect. 4 shows that there cannot be any metaphysical-invisible waves. There is no wave-particle duality, there are only particles (quantum abstract atoms).

Why do some physicists go against the Copenhagen position that the wave function does not, strictly speaking, exist? We can find the answer if we consider what Greene-certainly one of the most famous and widely respected physicists today-says in the very next paragraph of Greene 2004 after the passage we just gave: "Thus, the success of quantum mechanics forces us to accept that the electron, a constituent of matter that we normally envision as occupying a tiny, pointlike region of space, also has a description involving a wave that, to the contrary, is 
spread through the entire universe." ${ }^{209}$ (My emphasis.) As mentioned above, this is the metaphysical theory of wave-particle duality, and we can see that it is also connected to the idea that there all particles are metaphysical (unobservable) waves, in addition to empirical points (points on the screen). Notice the way that Greene uses the word "thus" in his passage. If one looks at the page that the passages are given, it appears that this word "thus" connects from the mere metaphysical assertion that any particle, in addition to being a point, is a probability wave spread out through the universe. Greene's reasoning (from pages 88-90) is as follows: (1) Born theorized that there are probability waves as the best description of the collapse of the wave function; (2) this is the best description of quantum mechanics; (3) this indicates that there are metaphysical wave aspects to every point-particle, and (4) "[t]hus, the success of quantum mechanics forces us to accept that the electron... also has a description involving a wave..." (page 90, my emphasis) Notice that, using some of Greene's own words, we see that "description" "forces us to accept" a metaphysical wave. But there is no reason I can see why description does this. Description can be an instrumentalist tool, of course, without denotation. Greene goes to admit that waves are merely instrumentalist when he tells us that the "utility of quantum mechanical probability waves to predict and explain experimental results has been established beyond any doubt." 210 But then, surprisingly, and in disagreement with what was just written, he writes:

Yet there is still no universally agreed-upon way to envision what quantum mechanical probability waves actually are. Whether we should say that an electron's probability wave is the electron, or that it's associated with the electron, or that it's a mathematical device for describing the electron's motion, and that it's the embodiment of what we can know about the electron is still debated. ${ }^{211}$

This seems a rather shortsighted discussion, which is coming from one of the world's most famous physicists. I put the issue in this harsh way not to wastefully produce slander, but rather in order to make a certain point, one which philosophers often agree with: physicists very often do not want to do philosophy, they are uncomfortable interpreting their work for the public, and they are more comfortable with mere data and experimentation. These are dangerously broad generalizations, of course, but they are worth stating it seems since they may be able to give us some indication as to why there exist all the oddities we are going through in this article that occur when physicists develop philosophy (such as developing the wave-particle duality paradox, etc.). Returning to Greene, in just three pages of his book The Fabric of the Cosmos, he tells us that quantum waves do and do not exist, and that they instrumentalist and this instrumentalism forces us to acknowledge that they are mind-independently real (i.e. non-instrumentalist) - two claims which are clearly contradictions in terms. But, against Bohr, Greene's account is the account of where wave-particle duality arises. I see no way to avoid the conclusion that it appears that the entire "paradox" is not well established as a description of how nature really is. ${ }^{212}$ It seems to me safe to assert that we are given absolutely no reason why there are extended probability waves-which

\footnotetext{
209 Greene $(2004,90)$.

210 Greene $(2004,91)$.

211 Ibid.

212 Murdoch appears to entertain the idea that a no-nonsense empiricism that is not averse to instrumentalism indeed "need not be troubled by wave-particle duality." (Murdoch 1987, 27) 
would go against the principles of mereological nihilism-and instead we can agree with Stenger (cited in 2.4) that there is no wave in the wave-particle duality. If there is anything that physicists should know, it is that utility of description do not mean reality is this or that way beyond the description, lest we forget what happened with the blunder of the ether. ${ }^{213}$ I will discuss wave-particle duality more in the next section.

\subsection{Quantum atomism}

As mentioned elsewhere in this article, quantum physicists are often very eager to distance themselves from the philosophy of atomism. ${ }^{214}$ One of the principle ways they do so is by using the metaphysical thesis of wave-particle duality. Consider this passage from Omnés:

Let us look at an object,... [such as] a billiard ball... Nothing could be simpler for us: everybody has seen such an object, and as you were reading the first sentence of this paragraph, an image of the ball formed in your imagination. Less than a century ago, a physicists would not have thought otherwise, except for some additional precision... To an atomist, the ball would have appeared as a dense pack of atoms, with each atom being imagined as another very small kind of ball.

There is nothing resembling all that in quantum physics. The physicist does not start from the idea of the ball as an assemblage of a colossal number of atoms, but this idea is immediately replaced by a wave function which depends on as many variables as there are electrons and atomic nuclei in the ball. ${ }^{215}$

Omnés only considers one variety of philosophical atomism, as if there can only be one variety. He assumes atoms can only be classical (non-quantum) extended solids (Democritean atomism), and he does not recognize that there are many varieties of philosophical atomism that have emerged through the millennia, such as Buddhist

213 At many points in this article, such as was just argued, I am giving evidence for the thesis that the quantum paradoxes do not exist: there are no paradoxes, there are only erroneous interpretations. Some philosophers, like Quine and Putnam, put enormous importance on these quantum paradoxes, using them to make conclusions in their philosophy, based on the literalness of the paradoxes. But if the reasoning I am presenting is correct, and there are no quantum paradoxes, it would draw the conclusions Quine and others made into question. For example, it would bring into question this claim by Quine:

$[\mathrm{N}]$ o statement is immune to revision. Revision even of the logical law of the excluded middle has been proposed as a means of simplifying quantum mechanics; and what different is there in principle between such a shift and the shift whereby Kepler superseded Ptolemy, or Einstein Newton, or Darwin Aristotle? ("Two Dogmas of Empiricism," page 43).

214 For example, consider this passage from the famous physicists, Davies and Brown:

The discoveries of the electron and of radioactivity, the success of Planck's quantum hypothesis and the inception of Einstein's theory of relativity swept away the entire basis of NewtonianMaxwellian physics. Newton's laws of motion and his commonsense assumptions about space and time were abandoned. Even Democritus' atomic hypothesis had to be replaced by a more subtle and complex view of the microworld in which atoms could no longer be regarded as indestructible particles with a well-defined position and motion. It became apparent that the foundations of classical physics had collapsed.

By about 1930 their place had been taken by new theoretical schemes: quantum mechanics, the general theory of relativity and a more elaborate model of the atom. (Davies and Brown 1988, 4)

Of course the discovery that the atoms that scientists study having parts is not threat to the philosophy of atomism, as that merely pushes back the issue to the parts of the atoms (electrons, etc.) (and thus show that scientists' atoms do not exist).

215 Omnés (1999b, 148). 
atomism, which is virtually identical to quantum atomism. Also note the other assumptions that Omnés makes: there is a wave to consider in the metaphysical wave-particle duality assumption, and the wave-particle duality is also a problem for atomism. But in this subsection I have shown that there are reasons to reject wave-particle duality, and to only accept the I-empirical items (quantum abstract atoms), and not the metaphysical inventions (waves) associated with the I-empirical observation of those atoms. And these assumptions therefore, should merely be rejected. If there is no wave to wave-particle duality, then there are only the particles. And since we are finding physicists to be describing many of these as partless particles, I do not see a way to avoid the conclusion that they are obviously atoms. This is why I come to the conclusion that mereological nihilism is a theory of quantum atomism. Although I am sure critics of this article may feel inclined to attack the anti-metaphysical mood of the reasoning here, but the reason there is such a mood is because metaphysics certainly appears to lead to errors in reasoning, and I know of no better examples of this than wave-particle duality, which literally leads to the espousal of: reality $=$ contradiction.

The particles giving rise to a wave pattern is no more than a series of simple events giving rise to a predictable repeatable pattern over some period of conceptual time. More specifically, the wave-particle duality paradox came from the two-slit experiment, which, very simply put, reveled that there were specific quantum experiments that could be done that show quantum abstract atoms behave as follows: (1) there are unconnected events (firing electrons one-at-a-time) (2) which give an apparently random outcome (unpredictability of where they will hit on the screen), (3) which give rise to an overall ordered pattern when many random firings are compared. The overall pattern in (3) has led physicists to maintain that the items of (1) have that pattern "in" them somehow. But this is analogous to maintaining that the single hydrogen atom has the whole wave's nature "in" it. Even physicists' own description of the interference patterns in wave mechanics does not allow for this, since waves are understood to be produced when particles are "together," not when they are "alone." Further, there are many instances in DIV2-experineced reality, where we see the 1-2-3 pattern just described in nature, but where it is not asserted that the individual items in (1) individually have the pattern observed in (3) "in" them. To give just one example: solar flares appear to occur (somewhat) randomly (less randomly now that we are understanding them better), but their overall activity forms an ordered pattern (reaching what are called "peaks"). ${ }^{216}$ But astrophysicists don't assert that the individual solar flare has the pattern-nature "in" it. Countless other examples could be given.

The real mystery is why our DIV2 or DIV1 experiences regularly appear to us in patterns at all. In any series of experience impressions - wave or otherwise-reality presents itself through instantaneous moments that do not have any known connection to the past (see Sect. 4.5), but for some reason give rise to a pattern when we experience many of the instants. The two-slit experiment is just one account of how seemingly random and unconnected events present themselves as patterns to DIV2 experiencers through time and via memory (see Sect. 5.2). Apparently virtually all moments of our lives are this way, where unconnected instants create patterns in our

216 See the June 2, 1999 NASA story: "Solar Flares Show Their True Colors. New research points to a common mechanism for spectral behavior in Solar Flares." <http://science.nasa.gov/newhome/ headlines/ast02jun99_1.htm>.

Springer 
memory. The mystery is why we have these patterns, whether in waves from individual particles, or due to anything else.

In exploring the thesis of quantum abstract atomism, quantum uncertainty about location and motion is a relevant issue to discuss. Physicists typically tell us that quantum uncertainty about location and motion reveals that quantum objects have some sort of extendedness (magnitude, size) due to the fact that their location is smeared. Smearing is understood when we understand that an object only has a precise (point-sized) location if it is unmoving, and any object in motion thus cannot be described as being unsmeared and at a precise location. The various notions of quantum uncertainty involve "[m]easurable physical properties like color and hardness are said to be "incompatible" with one another, since measurements of one will (so far as we know) always necessarily disrupt the other." ${ }^{217}$ Regarding motion and location, issues to do with uncertainty lead physicists to conclude that particles are, for example, blurry cloud-probabilities. ${ }^{218}$ The smeared, blurry, streaked particles clouds or lines are not considered by physicists to be products of erroneous perception, but rather to be real extended objects: the point-electron (somehow) smears to form an $n$-dimensional object $(n>0)$. How is an electron, which is a structureless point, extended (not a point)? Also, the reasoning of Sect. 4 specifically showed that extended partless objects do not exist. Therefore, there has to be a problem with the conceptual analysis of imagining that quantum uncertainty leads to the idea that an electron or any quantum abstract atom can smear to give rise to an extended item. It is not that there is not uncertainty in measurement and quantum physics, the specific issue the argumentation in this subsection and in Sect. 4 is that quantum uncertainty cannot lead to the conclusion that there are extended objects in quantum reality. For that reason, if in electron microscopy, for example, an extended electron cloud is observed, then that must be a DIV2 illusion, somehow created by the measuring of the electron's instances. The Heisenberg uncertainty principle shows us that if we want to know the velocity and location of an object simultaneously, that measurement will only give rise to an extended object-above referred to a smeared object. This uncertainty specifically stems from measurement, not from the real characteristics of a particle. This is a controversial issue, since physicists claim that the smearing is not a product of measurement error or limitation, but rather the electron is really extended by its being smeared. In discussion of Heisenberg's uncertainty principle, consider this passage from the later chapters on quantum physics in the fourth edition of one of the most widely used introductory physics text books, Fundamental of Physics (Extended Version), ${ }^{219}$ by Halliday, Resnick, and Walker, page 1169: What Feynman is saying, in effect, is: "Think in terms of matter waves. Throw out the notion of the electron as a tiny dot. When you want to think of electrons, do so statistically, being guided by the probability density of the matter wave." In effect, this is a plea for metaphysical analysis of quantum wave metaphysics, since priority is given to metaphysical wave over I-empirical particle. Physicists reject the idea that the electron is like, for example, a glowing bullet shot through a dark room makes a line-smear. Such a line does not exist, strictly speaking, outside of consciousness, and it is generated by the inability to see finely enough to decipher the smaller bullet locations while it traveled. The line only

\footnotetext{
217 Albert (1992, 6-7).

218 Omnés (1999b, 149).

2191993 (1974). New York: John Wiley and Sons.
} 
exists due to the inability of the DIV2 perceiver (perceiver $=$ mind-dependent generator of surfaces and colors) to see the motions of the bullet on a more finegrained level.

There are numerous fatal problems, however, with imagining that the smear is not like the streaking bullet (according to which, the steak is generated by consciousness, and the real item, the bullet, is somewhat hidden behind this illusion). If we reject the glowing bullet model of the smeared electron, we must take up the ideas that the electron is really extended, and the extended image generated on the computer screen in electron microscopy is a real three-dimensional solid. But the problems with extended electrons are well-known. Firstly, extended objects have parts, as we saw above. Not only would such a view fall under the attack of the reasoning given in sections above, where we found that composite and extended objects do not exist, but also there are plenty of problems with the extended electron that physicists have pointed out for us, which are enough to toss the idea of the extended electron. First, extended electrons are impossible since their halves would repel and the extended electron could only explode. ${ }^{220}$ Also, being a Democritean atom, an extended electron would be in violation of Einstein's relativity theory. ${ }^{221}$ And the electron, having parts due to its extension, would violate its empirical foundation of being revealed it to be unstructured (see Kane's quote in Section 1), and would also, as stated, being in violation of every bit of reasoning in Sects. 3 and 4 above. ${ }^{222}$ For these, and perhaps other reasons not even listed here, I do not see how I am supposed to accept the conclusion that there can be an extended, solid, electron-smear. If an image is generated of an electron probability cloud in a computer screen, I do not see how I am to avoid the conclusion that there is no real solid item that is not an illusion created by a computer screen and/or DIV2 consciousness. And I do not see how I am to avoid the conclusion that electron smear is an illusory (mind-generated) smear, just like the glowing bullet's smear. I do not see how it is not the case than, as Green writes: "[w]hen we locate an electron, we always find all of its mass and all of its charge concentrated in one tiny, pointlike region.",223

But there are other problems with uncertainty beyond those just listed. Physicists are endlessly describing their theories in terms of space-which is a metaphysical idea. If there is quantum uncertainty about location and motion, this would be a primary motivation for physicists to find quantum reality to be not to be atomicious: in this article, through philosophical reasoning, I have found that quantum atomism can only involve immaterial point atoms that flash in and out of existence, and extended cloudy quantum objects are the antithesis of such a philosophy of quantum atomism. ${ }^{224}$ But it is not hard for us to see the fallacy and vacuousness regarding quantum uncertainty about motion and location. First, notice that the concept of location only has meaning if relationalist or

\footnotetext{
220 This is a widely discussed issue. For just one example of a good account of this, see Davies (1984, 107-108), who is a well-known physicist.

221 Davies (1984, 107).

222 Greene discusses problems similar to these regarding extended and/or spread-out electrons on page 88 of 2004 .

223 Greene $(2004,88)$.

224 The abstract atomism involved with the R-theory of time in Grupp (2005a) also involves a complete rejection that there is quantum uncertainty at the level of ultimate particle reality, and of the idea that uncertainty leads to extended and/or "cloudy" quantum objects.
} 
substantivalist space exists. As discussed above, labeling objects as being at locations in space is going beyond the empirical into the paradoxically metaphysical. Without locatedness-which no particles have, according to the conclusions of this article-there is no uncertainty about location and motion. The glowing bullet is a construction of DIV2 experiencing, due to the imprecision of empirical experience, not the way the bullet is. Similarly, there are no quantum probability clouds without locatedness to make particle uncertain. The issue is not whether a quantum abstract atom has definite position or not, since it has no location at all. To get a further idea of where the metaphysicality of quantum uncertainty about motion and location comes from, consider how it comes from the wave notion, which we can see in a passage from Greene, "Uncertainty is built into the wave structure of quantum mechanics and exists whether or not we carry out some clumsy measurement." 225 In the sentence after this on the same page Greene mentions how quantum uncertainty is due to probability waves, the very ones we have found above to be metaphysical fabrications. Nobody has ever observed the particle clouds that physicists so often refer to, and if there are no probabilities waves or spatial locations, there is no way that such extrasensory and metaphysical quantum probability clouds can exist. And if they do not, there is no reason to maintain that quantum uncertainty threatens quantum atomism.

I am confident that some physicists will employ a familiar ad hominem attack against the mereological nihilist interpretation of quantum mechanics since it is a no-collapse interpretation, since it is not so respectful to Heisenbergian smear-cloud particles, it is waveless, there are no probability clouds, and so on. But the reasoning above dictates that I go this route, and of course there are interpretations of quantum mechanics that do not involve collapse, ${ }^{226}$ that do not involve respect to waves, or to probability clouds.

In conclusion, I do not see how I am to accept the idea that there are quantum paradoxes, when, as discussed, the paradoxes themselves appear to be not well established. Given the empirical data, and given the logical reasoning of this article, both of which are in agreement, I do not see how I can avoid the conclusion that the quantum paradoxes are a hoax, and that what is really waiting to be discovered is an answer as to why quantum reality is how it is.

\section{Scientific evidence for mereological nihilism: conceptualism, nihilism, forces, and the quantum revolution}

Mereological nihilism is a remarkable philosophical position due to the fact that all of the empirical objects that humans ordinarily perceive in their daily life (such as trees, lions, mountains, or any of the macroscopic objects that can be perceived by their surface and/or a color) are objects that have extension and parts, and thus do not exist, due to the reasoning found in the above sections, and if mereological nihilism is a correct theory. If mereological nihilism is a correct theory, it means that nearly all of the beliefs humans ordinarily hold about reality are incorrect, and there is something completely misleading about all of the ordinary perceptions that humans typically

\footnotetext{
225 Greene $(2004,98)$.

226 For example, see Albert, David Z, and Loewer, Barry. 1989. “Two No-Collapse Interpretations of Quantum Theory. Nous 23 169-186.
} 
have about reality in their daily life. If mereological nihilism is a correct theory, then nearly none of our science (except for some research areas in quantum physics) is correct, and nearly all of our language is incorrect: scientific and ordinary language are wrong (they do not denote) since almost always about objects that do not exist.

If we magnify the extended matter that humans ordinarily (believe that they) perceive down to the quantum abstract atoms (electrons, quarks, etc.), we find that the perceived extended matter does not in fact possess any continuous spatial extendedness nor colorfulness. Also, during the indirect quantum observation of particles that scientists routinely carry out, they do not witness any metaphysical relations between the particles, such as those espoused by Newton, Faraday, almost any other pre-1900s physicist, and by multitudes of contemporary metaphysical realists. If particles do not touch or relate, then there is a failure in our only ways for describing how particles accumulate to give rise to the extended and/or colorful solid, liquid and gaseous objects humans experience empirically. It would seem that the quantum reality discovered in experimental physics is in strong agreement with mereological nihilism. For reasons given so far in this article, quantum science can be considered evidence for mereological nihilism and for the conceptualism (mental fabrication) of empirical experience. Petitot and Smith write:

The rise of mathematical physics has long been seen by many as dictating a dismissal of the phenomenal world - the world of macroscopically organized in objectual forms, shapes, secondary qualities and states of affairs-from the realm of properly ontological concerns and as dictating a concomitant 'psychologization' of phenomenal structures. ${ }^{227}$

The brand of extreme conceptualism that mereological nihilism leads to can be defined as follows: all of the surfaces and colors humans experience empirically are not objects that are out in the world, but rather they are mental constructions created in the act of being conscious. Mereological nihilism appears to give evidence for this variety of conceptualism, since in a mereological nihilist reality, solids, liquids, or gases, and any conceivable macroscopic objects that have a surface and/or a color do not exist. Rather, they are concepts and ideas in our imagination, whereupon it would follow that ordinary life, and everyday empirical experience is no more than a stream of imaginal thought-experiences. The idea that surface and color do not exist except as mental concepts are not at all new to philosophy (even though it is not a position widely accepted in-and to some degree it has been banished from-philosophy in the contemporary era), which can be seen by considering a passage from Lockwood about Hume and Hobbes on the colorfulness of objects (one of the two ways we experience the external world-surface being the other):

Some philosophers, of whom Hobbes is one and David Hume possibly another, adopt an error theory of colour... That is to say, they hold that our prereflective, or at any rate pre-philosophical, conception of the material world is actually mistaken, in so far as it holds that physical objects really are coloured. According to the error theory, our tendency to believe that objects are

227 Petitot and Smith (1997, 233). In their article, Petitot and Smith goes on to argue against the position that quantum mechanics is strongly opposed to common sense. 
coloured is a consequence of an illegitimate projection on to the external world of things that are going on exclusively in our own minds. ${ }^{228}$

Quantum science has changed to become more and more of a mereological nihilist endeavor due to the past 100 years of revolutionary quantum theory. There was a revolution in science in the early 1900s, with the inverse-physics found in the discovery of the quantum world, Physicists such as Faraday, Newton, Einstein, and so on, before the quantum revolution, held that positions 1 and 2 referred to in Sect. 4.1 (contacting and/or relating between quantum particles and quantum abstract atoms) were responsible for the way in which quantum particles or philosophical atoms give rise to the empirical objects we perceive, but that is no longer the case. Physicists, before the quantum revolution, typically asserted that metaphysical relations (that we found to be impossible in Sects.4.3 and 5.1) are ultimately behind the descriptions of the structure of matter, space, fields and forces in their physics. Jammer writes:

With the rise of Newtonian dynamics and its interpretation along the lines of Boscovich, Kant, and Spencer, the concept of force rose almost to the status of an almighty potentate of totalitarian rule over the phenomena. And yet, since the very beginning of its early rise to power, revolutionary forces were at work (Keill, Berkeley, Maupertuis, Hume, d'Alembert) which in due time led to its dethronement (Mach, Kirchhoff, Hertz). This movement in mathematical physics, from the time of Newton onward, was essentially an attempt to explain physical phenomena in terms of mass points and their spatial relations. For it became increasingly clear that the concept of force, if divested of all its extrascientific connotations, reveals itself as an empty scheme, a pure relation. ${ }^{229}$

After the quantum revolution, when physicists leaned away from 1 and 2 (contacting and/or relatedness between particles), physicists still discuss atoms and particles as involving "connections" (and rather strange ones) between them, and they still even refer to them as forces and/or fields. But since the quantum revolution, now the forces and fields are not considered to be relations or metaphysical continuous connections between particles. Rather, the forces and fields are indirectly perceived by scientists through their measuring apparatuses to nothing but unattached, noncontacting, unconnected quantum abstract atoms, and no relations or connections of any sort are anywhere to be found in the I-empirical observations of the quantum world. Gribbin discusses this in a passage that is about the basics of what a field is according to the quantum mechanical framework:

In classical physics, a field is something which stretches out from an object and conveys a force (there are really only two forces in classical physics, gravity and electromagnetism). The force can be described in terms of ripples in the field, or waves. But in quantum mechanics we know that waves can be described in terms of particles. So the concept of a field in the classical sense is replaced by the concept of particles which carry forces as they are exchanged between other quantum entities. The classic example is the photon, which mediates the electromagnetic force... ${ }^{230}$

\footnotetext{
228 Lockwood $(1989,5)$.

229 Jammer (1999, 241-242).

230 Gribbin (1998, 316-317).
} 
From what has been discussed in this section, we can see that the idea is that reality is composed of interrelated networks of particles, or of continuous fields and forces between particles, is antiquated, and the most progressive view (progressive in scientific terms) appears to be one where reality is composed of unrelated and unattached particles, which is also the mereological nihilist position. However, many philosophers often still erroneously refer to the fields and forces of physics (e.g., gravity, electromagnetism, the color force, dark energy, etc.) as relations and continuous connections and metaphysical connections or forces between particles, and they often refer to them as if those forces, relations and connections are purely metaphysical entities. ${ }^{231}$

It is interesting to note how often philosophers still maintain that the "push-pull" forces between particles are describable as relations, when physicists have discovered them to be merely unattached non-touching quantum abstract atoms, whereupon it seems dubious to describe the forces as being in any way primarily metaphysical relations rather than merely what we have evidence for the existence of: namely particles! Hoffman and Rosenkrantz write:

According to currently accepted [science],... the adherence relation instantiated by parts that compose an actual mereological compound results from an equilibrium of attractive and repulsive forces of certain kinds among those parts. The particular nature of such an adherence relation is an empirical question to be decided by scientific investigation of the mereological compound in question. $^{232}$ (Emphasis added.)

Since science (quantum physics, specifically) appears to point toward the veracity of mereological nihilism, we should expect there to be a movement among philosophers who want to go beyond an evidential account in order to reject scientific and philosophical evidence, and in order to fulfill their desires for, and beliefs in, the flimsy veil of perception and, as stated in the introduction, to believe the world they perceive is more or less the way the reality really is. If the evidence for mereological nihilism is as strong as it appears to be in this article, we should expect to find such philosophers, in going outside of an evidential inquiry in order to fabricate theories that support their mere belief in the world they see, can only do so by constructing prephilosophical and/or prescientific theories. We will see below that this is what happens, and we know it because we will see that such philosophers admit it. In a passage about Jay Rosenberg, Markosian maintains that we cannot use science to aide in issues to do with composition:

The puzzling [thing]... is that Rosenberg seems to want to suggest that sciences such as microphysics, physical chemistry, and biology can help us to answer SCQ. This is puzzling because those sciences are, after all, empirical sciences, whereas a correct answer to SCQ would have to express a proposition that is necessarily true. For this reason, it is hard for me to see how exploring any of the sciences mentioned by Rosenberg could help us to find a correct answer to SCQ. ${ }^{233}$

\footnotetext{
${ }^{231}$ For example, Elder appears to make this error, in a passage about atomic rearrangement of an exploding building:

The microparticles... affected by the explosion call on relations of spatial proximity and electromagnetic charge (or, for quarks, relations with respect to 'colour') for their sufficiency to produce violent alterations in the microparticles of the second tier... (Elder 2003, 30-31).

${ }^{232}$ Hoffman and Rosenkrantz (1997, 83).

233 Markosian (1998b, 229).

Springer
} 
Here Markosian is admitting that science cannot tell us how atoms compose composites, and that if there is a solution to this problem, it will be found in philosophy, and it will be specifically found in metaphysical philosophy, since he is saying that it is not in the realm of the observable (it is not empirical/scientific) to find the answer.

It is by a prescientific and prephilosophical (i.e., non-scientific and non-philosophical) pursuit that one can deny mereological nihilism, given that the evidence for it appears to come on many fronts, as this article demonstrates. I will next discuss this prescientific and prephilosophical (non-scientific and non-philosophical) attitude that some philosophers exhibit.

\section{Attacks on mereological nihilism}

The few contemporary philosophers who mention mereological nihilism typically tend to describe it in derogatory terms, and via ad hominem and other informal fallacies. Consider what Hudson writes (in a rather placid passage, compared to others in cited in this article):

Nihilism [is].. roughly, the view that there are no material objects with proper parts. Nihilism is usually mentioned (as it will be here) only to be more or less immediately rejected. It earns a place among the popularly discussed theories primarily because it lies at one of the extremes along the continuum of answers... Nihilism is a non-starter. ${ }^{234}$

Hudson's comment is surprising, in light of the what I have written in the sections above. Perhaps Hudson is only referring to contemporary philosophers in his passage, and when he asserts that few philosophers have espoused the nihilistic position. In various epochs of the past mereological and compositional nihilism has been the mainstream position (Democritus and Greek atomism, or some accounts of the preclassical Indian Buddhism are two good examples; others were mentioned above; and even some physicists espouse mereological nihilism. A good example of a physicist who appears to hold a nihilistic or somewhat nihilistic position is Stenger. ${ }^{235}$ ) But I will next discuss that it is common for philosophers to misrepresent nihilism as an outrageous and radical philosophy, that can be rejected without need for an argument showing why it can be, and where it is demanded that it is "a non-starter", making it appear to be a philosophy that has no scientific evidence behind it, no philosophical justification, and as if it is a philosophical position that no philosophers have ever espoused. As I show in this article, nothing could be further from the truth.

\footnotetext{
${ }^{234}$ Hudson (2001b, 81-82).

235 This is what Victor Stenger refers to as a "particle reality":
}

The standard model offers a picture of elementary quarks and leptons, interacting by the exchange of a set of elementary bosons... In this book I am making the unremarkable suggestion that the quarks, leptons, and bosons of the standard model can be safely regarded as elements-perhaps the only elements - of an objective physical reality... The alternative ontology in which continuous fields are "more real" than particles was discussed in the previous chapter. First, we saw that a dual ontology of fields and particles, as existed in the nineteenth century [physics], contradicts the oneto-one correspondence between particle and field in modern quantum field theory. We can have either a reality of fields or a realty of particles (or other localized objects). We cannot have both without asserting some new physics not described by relativistic quantum mechanics. Such an assertion is uneconomical-not required by the data... Second, we saw that any viable field ontology based on relativistic quantum fields necessarily entails a Platonistic view of reality. (Stenger 2000, 253-254.) (Emphasis added.) 


\subsection{Two sorts of empirical experience: ordinary and scientific}

Many physicists and philosophers (attempt to) reject mereological nihilism by means that are prescientific, and in the words of a few of them, prephilosophical. Markosian writes on pages $214-215$ of 1998 b that he will find in that article that the widely discussed answers to SCQ unacceptable. ${ }^{236}$ (It is interesting that he finds the given responses to the SCQ "unacceptable," since that appears to be a claim that mereological nihilism would so far be our best philosophical theory.) He also, however, maintains that he finds mereological nihilism to be unacceptable (pp. 219-221). But he only presents two objections to mereological nihilism: (i) according to mereological nihilism, "there are far fewer objects in the world than most of us take there to be." (220) And (ii) according to mereological nihilism, "there are no physical objects composed of many parts. So nihilism seems to entail that you and I do not exist. And we can't have that." (220) I take each of these claims to involve the same grounding: mereological nihilism disagrees with commonsensical intuitions and with ordinary empirical experience. But I will nevertheless treat each of these separately. (I do not discuss ii) until Sect. 8.2.)

As for (i), it is a claim that our intuitions about the world are trustworthy enough that we can use them to reject the scientifically oriented mereological nihilism. (This is, of course, diametrically opposed to the scientific method which was developed for us by Bacon and Descartes, where we are to let discovery guide intuition rather than intuition guide discovery-the latter of which was Aristotle's method that actually prohibited science for millennia, just as metaphysical opposition to mereological nihilism appears to be doing today, thousands of years after Aristotle.) This is the standard way the philosophers attack mereological nihilism: by pleading for respect of ordinary empirical experience, and of commonsense intuitions about reality. I will next show that they are acting in opposition to science and to what I will discuss is a refined empiricism. They are instead operating in accord with a non-scientific, commonsense empiricism (what could be called a plebeian and popular DIV2 empiricism). For these reasons, I will show that these philosophers are not presenting evidence and reasoning that attacks mereological nihilism. It is merely an urging to accept the intuitive view as merely given by common everyday DIV2 experience, which Markosian calls "pre-philosophical intuition at the start of 1998b, as if that it is a good thing to keep hold of when creating philosophical theories, rather than accepting a post-intuitive (scientific and logical) view. Science and reason have been much better in the past at informing us what reality is like than intuition has. (Examples are given below.) Intuition leads to quasi-Aristotle's anti-scientific investigation of nature, and science leads to an anti-Aristotelian investigation of reality. I understand Markosian's being troubled by the fact that ordinary empirical experience is in disagreement with

\footnotetext{
${ }^{236}$ He does not do this in order to show that mereological nihilism is the best theory due to the fact that compositional theories are "unacceptable." Rather, he does this merely to introduce his theory of "brutal composition," which is supposed to be an acceptable account of composition, but it is no more than a demand that we not discuss composition, since "brute" basically means "unanalyzable." This is an attempt to block philosophical analysis of something as important as material composition. This is a great example of how philosophers will often attempt to reject that there can be philosophical analysis of DIV2 reality when the going gets tough. All the aforementioned paradoxes basically enjoy this status among philosophers. This is also a great example of the dearth of philosophical theorization the theories of mereological and compositional philosophy give us in understanding reality: they are so vacuous that we have to resort to demanding that there is no explanation possible for composition, as if to demand that, composition just is, and you can't know how or why.
} 
the empiricism of quantum physics. But science is all about overturning (sharpening, improving) mere ordinary commonsensical DIV2-empirical experience, which tends to be crude and imprecise, plebeian, popular, and uninformed, ${ }^{237}$ and which tends to believe such things such as continents don't move (float) across the surface of the earth, or that the cosmic background radiation is not all around us, has no idea that neutrino particles far outnumber ordinary matter particles, has no idea that dark matter and dark energy exist, and is unaware that elephants are talking to one another (even though we can't here anything, when we are around them).

This prompts us to define two sorts of empirical experience: 1 . honed and scientific empiricism, and 2. ordinary and plebeian empiricism. I will name 1 and 2 as follows:

1. Non-scientific empiricism (NS-empiricism): this is plebeian, popular, unquestioning DIV2 empiricism that has not be refined by the shock of scientific discovery where the empirical experiencer tends to merely accept most of what is witnessed in this mode of experiencing (perhaps not mirages, but definitely a blue patch on their computer screen is not smooth, but is composed of individual lit units that have separations between them.)

2. Scientific-oriented empiricism (S-empiricism): this is DIV2 or DIV1 oriented empiricism that is discerning in a way that NS-empiricism is not, and which is critical in its approach of what is believed and accepted about what is experienced, and about what is to be questioned or rejected. NS-empiricism likely believes that a marble's surface is entirely smooth and shiny, but S-empiricists know that sufficient magnification of the surfaces is needed to find out if it is (and such magnification reveals that it is neither smooth nor shiny but is as jagged as a fractal edge.) I-empirical experience is a subset of these S-empiricist experiences (and as pointed out in 2.4, only the I-empirical experiences of quantum abstract atoms can be experiences of real objects).

I take the metaphysical realist to appeal to 1 (even though they may attempt to try tell us that they are aligned with 2), and I will show why below.

It seems that few scientific statements could be more obvious than this one: science is empirical, but the way a scientist empirically experiences can only change by the process and progress of science. For example, when one begins studying biology, one may think that "living things" denotes trillions of bugs and microbes and macroscopically visible animals (i.e., the sorts of living creatures one might be familiar with under NS-empiricism and before S-empiricism). But after the study of biology begins, and NS-empiricism begins to transform into S-empiricism, one will discover that denotation of "living things" just given false due to its truth value of false, and rather something like these statements may be one more in the direction of being correct:

One doesn't have to venture far into the underground for new discoveries. Step out into the backyard, for example, push your thumb and index finger into the root zone of a patch of grass, and bring up a pinch of earth. You will likely be holding close to one billion individual living organisms, perhaps ten thousand

\footnotetext{
237 I realize that I might risk sounding haughty, in a 19th century science sort of way, by referring to ordinary experience in this way, as plebeian, and as popular and uninformed, as compared to scientific empiricism. I do not mean these comments to be haughty and cruel, but rather to merely indicate that the greater population of the world has poor knowledge of science (and thus poor knowledge of reality), and thus has an empirical view based on that.
} 
distinct species of microbes, most of them not yet named, cataloged, or understood. Interwoven with the thousands of wispy root hairs of the grass would be coils of microscopic, gossamer-like threads of fungal hyphae, the total length of which would best be measured in miles, not inches. That's just in a pinch of earth. In handful of typical healthy soil there are more creatures than there are humans on the entire planet, and hundreds of miles of fungal threads. ${ }^{238}$

This example is meant to show how S-empiricism reveals the truth values of NS-statements to often be false (if the universe of discourse is only DIV2). In this example, it is presumed that the observer's concept of the quantity of living creatures on the earth changed (became larger), and by changing, the first belief was replaced by the second. Unfortunately, many realists (as defined above in the introduction) are not trained in the skeptical tradition of science, which involves an extreme undermining of NS-empiricism, and thus they are not aware of the fact that there are reasons to avoid NS-empiricism. (Mereological nihilism may be the ultimate undermining of commonsense and of NSempiricism, which undermines all the rest of science, where from the perspective of mereological nihilism, all of science except quantum physics involves statements about a reality that does not exist.) I do not see how I cannot avoid rejecting Markosian's (i), since it seems to be a variety of NS-empiricism, by Markosian's own admission at the start of, and throughout, his 1998b, where he shows that he is just trying to appeal to prephilosophical intuition, which must be some sort of commonsense, since both (i) and (ii) appear to common sense to be the grounding, rather than an S-empiricism.

\subsection{Markosian and selves or minds}

In exploring Markosian's (ii), it seems that Markosian is assuming both of the following: the self exists and that it is a composite item. All one needs to do is require an argument rather than an assumption or an account of intuition for these positions, and until they are given, we can disregard this as being an attack against mereological nihilism. In carrying out (ii), Markosian appears to be asserting that mereological nihilism can be disregarded since it cannot explain the "we" in Markosian's (ii), which is apparently the self or consciousness. (In a passage above we saw Hudson make a similar sort of claim.) But of course, as I will discuss in a section below, and as I will cite others discussing also: all of philosophy is plagued by a lack of coherence as to what mind and self are. What other branch of philosophy can explain it adequately? I am not clear why Markosian imagines that this is something only mereological nihilism is plagued by, as if the rest of philosophy is not. Aren't all philosophies stumped by the description of mind or self? So why is it only a problem for mereological nihilism? Markosian's attack against mereological nihilism is analogous to telling the mathematician that since nobody has a clear definition of whether or not numbers exist and/ or what the definition of an irrational number is, or what a complex number is, for some reason only ring theory in abstract algebra is in trouble, and the rest of mathematics is not-calculus and topology are amazingly unharmed. Or, as another example, Markosian's attack against mereological nihilism is analogous to telling the physicist that nobody has a clear definition of what dark energy or vacuum energy is, and nobody has clearly outlined what is really going on inside a black hole, or what the nature of Big Bang is (all base-level areas of physics, which is defined as the study of

238 Wolfe $(2001,1)$. Wolfe is a biology professor, and this is the first paragraph of Wolfe's book.

Springer 
energy), but only one small fringe area of physics, such as constructing experiments to verify electron entanglement, is in trouble rather than the entire field of physics. From the perspective of the DIV2 conscious experiencer, who has no idea that the surfaces and colors experienced are just concepts, and who describes her reality in terms of DIV2-oriented logics rather than quantum logics, Markosian's passage involves pointing out a problem with the whole (a DIV2 conceptual whole) but maintaining that only a part (a tiny DIV2 part) has a problem, which seems to confuse the DIV2 concept of part and whole.

Consider again the passage from Ned Markosian in an article of his where he discusses mereological nihilism (in this passage he uses "Nihilism" to be synonymous with "mereological nihilism"):

But there are serious difficulties associated with Nihilism. One of them concerns the fact that you and I are surely physical objects composed of many parts, if we exist at all; but according to Nihilism there are no physical objects composed of many parts. So Nihilism seems to entail that you and I do not exist. And we can't have that. ${ }^{239}$

Similarly, Peter van Inwagen, who some philosophers consider to be, to some degree, a mereological nihilist, however attempts the same attack against mereological nihilism as Hudson and Markosian when he maintains that "[n]ihilism is wrong because we are living, thinking animals, and composite objects therefore exist." 240

But van Inwagen, Markosian, Hudson and others do not offer us more specific argumentation for their position than this. For example, they do not show us, as just written, why and how we can conclude that the mereological nihilist's difficulties in explaining consciousness and personhood in a mereological nihilist reality reveal that mereological nihilism should be rejected, rather than just the less aggressive conclusion that personhood and consciousness cannot be nervous system emanations - which of course the mereological nihilist will enthusiastically agree with.

The passages from Hudson, van Inwagen, and Markosian (many others could have been mentioned regarding this issue) rest on the assumption that consciousness and/or personhood cannot be explained by mereological nihilism, and thus mereological nihilism is in trouble. Focusing just on Markosian's passage-the first passage we gave from the set from van Inwagen, Hudson, and Markosian-this is the structure Markosian's "reasoning" on this issue:

There is an all-important unsolved problem $p$ at the heart of popular/important theory $t_{m}$ of academic discipline $d$. $p$ reveals that only theory $t_{f}$ of $d$ should be rejected, and not $t_{m}$ and $d$.

Following Markosian's passage at involves these values of $t_{m}, p, d$ and $t_{f}$ :

$t_{m}=$ philosophy of mind and consciousness,

$p=$ explanation of consciousness or mind,

$d=$ philosophy, and

$t_{f}=$ mereological nihilism .

239 Markosian (1998b, 220).

240 Van Inwagen $(1993,684)$. 
It appears that this passage involves a number of informal logical fallacies, such as missing the point (expected conclusion not the one given: why is only mereological nihilism $\left(t_{f}\right)$ threatened rather than all of philosophy of mind $\left(t_{m}\right)^{241}$ or all of philosophy $(d)$, since these all need a consistent theory of consciousness?), appeal to ignorance (nobody has proven mereological nihilism can explain consciousness therefore there is no mereological nihilist reality), suppressed evidence (Markosian fails to reveal how all philosophies of mind, and all of philosophy in general, involves $p$, and I cannot see how to avoid the conclusion that it is through such suppressed evidence only that he can drawn the conclusion that he does), false dichotomy (either mereological nihilism has an explanation now for consciousness and mind or it is to be rejected-a third possibility, such as that mereological nihilism may be able to explain consciousness in the future, is ignored), red herring (rather than discussing the problems consciousness poses to mereological nihilism and to philosophy, and rather than explaining how they cannot explain consciousness and what should be done about it, we are distracted by the insignificant and fallacious such claim that this supposed to be a specific and fatal problem for only mereological nihilism), and, of course, begging the question (key premise is omitted, namely, the premise showing why only $p$ has a connection to $t_{f}$ in showing $t_{f}$ has some sort of specific problem due to $p$ : "only $t_{f}$ specifically involves $p$ because...").

(i) and (ii) are all Markosian (and the other plethora of anti-nihilists) have presented as (alleged) evidence against mereological nihilism: mereological nihilism disagrees with NS-empiricism, and it is not (yet) known how there can be consciousness in a mereological nihilist reality. Since that is all that is presented against mereological nihilism, it is safe to maintain that opponents of mereological nihilism do not have any real means of finding mereological nihilism "unacceptable," as Markosian puts it (1998b, 214-215). The analysis here from Markosian shows us how some philosophers are willing to avoid philosophical

\footnotetext{
241 This is my understanding of the philosophy of mind: it is agreed upon that consciousness and mind are not explained by any of the theories of mind and consciousness offered to us. Here's how McGinn puts the issue, in the first lines of his 1991 book (and where if one peruses the pages surrounding this passage, it appears that McGinn is using "mind-body problem" as synonymous with "consciousness"):
}

We have been trying for a long time to solve the mind-body problem. It has stubbornly resisted our best efforts. The mystery persists. I think the time has come to admit candidly that we cannot resolve the mystery... How can our technicolour phenomenology arise from soggy grey matter?... How could the aggregation of millions of individually insentient neurons generate subjective awareness? We know that brains are the de facto causal basis of consciousness, but we have, it seems, no understanding whatever of how this can be so... Somehow, we feel, the water of the physical brain is turned into the wine of consciousness, but we draw a total blank on the nature of this conversion. Neural transmissions just seem like the wrong kind of materials with which to bring consciousness into the world... (McGinn 1991, 1-2)

Also see a passage form Chalmers given in Sect. 7 below. There are many passages that could have been given by the famous philosophers of mind that directly indicate, or strongly imply, that current options given to us for explaining consciousness and mind are not sufficient.

Lastly, regarding McGinn's passage, it seems, suggest that brains do not support or generate consciousness. Quantum abstract atoms are no better candidate, at least as far as I can tell given the level of research and theoretical development that exists for panpsychism and quantum consciousness. This quite speculative conjecture is, however, of not help, as we will see in Sect. 7, since atoms seem to be no better candidates than neurons as producers of consciousness. 
analysis (which is based on inference and reason) in favor of their intuitions and their trust in common sense (NS-empiricism). ${ }^{242}$ Markosian even admits that this is what he is doing (that he is putting intuition over philosophical analysis) in a passage where, after presenting the scant "evidence" against mereological nihilism he writes:

I think that Nihilism remains extremely counter-intuitive. Speaking for myself, at least, the relevant intuition... is that there really are such composite objects as starts and chairs... That is, according to my intuitions, there simply are far more composite objects in the world than Nihilism allows. This seems to me to be a fatal objection to Nihilism, and I conclude, on the basis of this objection, that Nihilism is not the correct answer to SCQ. ${ }^{243}$

242 This is also very common in other areas of metaphysics, which are replete with paradox, but which are nevertheless embraced by some philosophers, especially metaphysicians. To just give one example, in Grupp (2005a) I specifically showed how persistence through time is impossible, how philosophers know there is no solution to this paradox that has yet been offered, and that an Rtheory of time avoids all of these issues, and an R-theory of time is needed to be in accord with science and philosophy, instead of mere NS-empiricism, commonsense and intuition. But the point is: it is interesting how willing some philosophers are to being friends of intuition over philosophy, and paradox over consistency, as this example—one of many-illustrates.

Instead of mereological nihilism, contemporary philosophers (namely realists) commonly hold one of the two widely popular metaphysical realist theories: the bundle theory or the substance theory. Both of these also have problems of being counter-intuitive and not explaining consciousness, just as Markosian and others claim is the case for mereological nihilism. For example, the bundle theory describes all objects as being collections of non-physical items so it is unclear how this non-physicality gives rise to the physical DIV2 reality (Hoffman and Rosencrantz chapter 1, section 4), and there the properties must flash in and out of existence every time there is change, and where the properties are to come into existence out of nothing whenever there is change (for example, if bundle B has properties $\mathrm{F}, \mathrm{G}, \mathrm{H}$, and $\mathrm{I}$ at time $\mathrm{t}_{1}$, but gains properties $\mathrm{J}$ at time $\mathrm{t}_{2}$, where did $\mathrm{J}$ come from? What caused it to come into existence?). As for the substance theory, it involves describing all items of the universe as involving a metaphysical, invisible, non-empirical items to be the "holder" or properties (usually called a thin particular, a thick particular, or something else) that ultimately in some way is a propertyless item that somehow is the holder of properties. Additionally, substance theory appears to be entirely in contradiction to the empirical particulate manner in which reality is observed to be in chemistry and physics (see Davis 2006).

${ }^{243}$ Markosian (1998b, 220-221).

In the next section, which is on van Inwagen's semi-nihilistic metaphysics in Material Beings, Markosian presents the very same "objection" to van Inwagen's theorization.

Insofar as this is one of my fundamental intuitions, I take the inconsistency of [van Inwagen's theory]... with this intuition to e the basis for a fatal objection to [van Inwagen's theory]. (221-222)

It is as if Markosian is indicating that intuition wins over philosophical analysis, and thus intuition, not argument, determines philosophy. I maintain this because it seems that Markosian is even positing that pre-philosohphic intuition wins over argument, because the very "intuition" Markosian is discussing on pages $220-222$ of $1998 \mathrm{~b}$ is "the intuition that there really are stars, chairs, and bicycles." (222) This is the groundwork Markosian lays at the outset of his article about prephilosophic intuitions. (211) So it does seem that Markosian is maintaining that intuition is stronger than reason and argument. But obviously intuition can be ever-so-wrong (the famous Harvard paleontologist Stephen J. Gould once said: "Science has not been kind to common sense.") Maintaining Markosian's position is analogous to maintaining that if a theory, theory $\mathrm{x}$, is in disagreement with one's pre-philosophic intuition then theory $\mathrm{x}$ is fatally flawed. But there are obviously many place-holders of " $x$ " that would show Markosian's trust in pre-philosophical intuition to be quite in error. For example, consider that theory $\mathrm{x}=$ plate tectonics. Not long ago at all, geologists once intuited so assuredly that plate tectonics was an inane theory (how could continents be moving?). 
"Relevant intuition," in the NS-empiricist way Markosian is using the word, might also inform us that tables are motionless, that bricks are solid, that boiling water is primarily chaotic rather than organized, that one stick is longer than the other in the Möller-Franz Illusion, that holes exist (such as the holes in a sponge, a keyhole, etc.), and that water waves involve water moving horizontally and not only vertically. Since "relevant intuition" obviously varies from person to person, and can even become inverted in a single person over time (for example, consider how fast Einstein's ideas radically changed the conceptions of people on basic issues about reality and how they experienced their world), unlike the selfchecking nature of science (philosophy is also supposed to involve a self-checking nature), intuition is quite free and malleable. Depending on who one is listening to, and regarding the following DIV2 concepts, "relevant intuition" may even inform us that humans are not animals, the universe is expanding, that some people can walk on water, and that militaries must invade other countries. The point of these examples is not to be fanciful and extreme or to push a biased position, but rather to show how serious ignoring reason in favor of intuition can be. In the case of the last example (militarism), favoring "relevant intuition" (to use Markosian's phrase, and where relevant of course means something more like "caused," in the DIV2 sense of the word) and ignoring reason can lead to unfathomable suffering for children and families, and decades or centuries of revenge and remorse. So considering Markosian's passage is no small issue when we see that he is not following philosophical reasoning or scientific discovery, but rather is following "relevant intuition," in his words, and that is why I assert that he is avoiding philosophical and inferential analysis, and instead he is promoting a position that is non-philosophical intuitive analysis, or prephilosophical, analysis. This is the mainstream "philosophical method" for metaphysical realists. He even implies himself that his article is based in pre-philosophical intuition rather than philosophical analysis, since at the beginning of his article on "brutal composition" he maintains that "[a]ccording to standard, pre-philosophical intuitions, there are many composite objects in the physical universe. There is, for example, my bicycle, which is composed of various parts." ${ }^{244}$ Throughout his article, he only relies on this intuition to give his "philosophical" positions (which appear more like religious positions, since they are based on intuition rather than on intellection, and on preference rather than inference), such as when he presents "evidence" against mereological nihilism, and in this passage given in the previous sentence he maintains that this same intuition is pre-philosophical, and for that reason, Markosian's attack is not an instance of philosophical reasoning, and mereological nihilism is unharmed.

\subsection{Attacks on mereological nihilism due to conflict with ordinary empirical experience}

I will next further discuss the type (i) attacks against mereological nihilism, where it is alleged that mereological nihilism is criticized for not matching up to ordinary $\mathrm{m}$ empirical experience (NS-empiricism). Most attacks on mereological nihilism, as can be seen in Hudson's passage at the start of this section, is merely due to the fact that people have sense experiences that they do not agree with

\footnotetext{
${ }^{244}$ Markosian (1998b, 211).

望 Springer
} 
mereological nihilism. Consider another example, in a passage from Hossack, a metaphysical realist:

...it seems there are at lest three distinct ways in which a complex might arise from a multiplicity of more elementary things: it might arise by composition into a mass, or by composition into a composite individual, or by collection into a set. But atomists deny that composition or collection ever really occurs, for they deny that there really are such particulars as masses, composite individuals, or sets.

An argument from Leibniz' Law can be used at this point against atomism. In the case of masses, we would argue as follow. A mass of matter is made up of particles. For example, we know that if there is some water, then it is composed of many water molecules. Now it is surely true that this water is wet. But of course no water molecule is wet. Hence "this water" must refer to something other than the individual molecules. Conclusion: it refers to this mass of water. Therefore, masses exist. ${ }^{245}$

In Hossack's passage, his position rests entirely on this demand: "...surely it is true that this water is wet." (Notice, in a similar fashion as with Markosian's and Hudson's passages above that Hossack gives no argument for this claim, and it therefore takes the form of a demand, rather than a philosophical position which involves an inference to a conclusion-the philosophy is merely based in mere trust that all will agree that the senses are infallible on such matters, which seems nearly shocking, since there is a long tradition of philosophers and scientists who would disagree.) Hossack uses this demand to presume that wetness can only be "in the water," rather than being a secondary quality (and thus in the mind), and it is as if this is avowed to be enough to reject mereological nihilism. But as implied above, once one makes this simple and seemingly harmless and even scientific move of questioning NS-empirical experience, one can easily notice that our sense experience is inadequate. So if, like Markosian and Hudson, only the crude and uncritical NS-empiricism is used to challenge mereological nihilism, then this objection does not seem to be an objection at all. Hossack's passage does not question his non-scientific empirical experience (NS-empiricism), and thus it seems that "it is surely true" that there is wetness, rather than there being a simple misperception, just like one has a simple misperception when they look at a red picture in a magazine and believe it to involve areas of continuous (unbroken) red or other colors patches rather than red or other colored pixels that have gaps between them (merely getting a magnifier out could correct the erroneous perception belief), or just as when one has a simple misperception when one sees a cloud and believes it to be a continuous (unbroken) while blob rather than detached and non-touching water droplets.

As we have seen, it is not difficult to see that the senses are plagued with inaccuracies, and we do no need to use quantum physics to see why. When we look at a piece of ordinary white paper with the naked eye, it appears smooth, white, spatially extended, and whole, and it appears to have no discontinuities or interstices across of its surface. For those reasons, and since there are no visible interstices, it appears to

245 Hossak (2000, 412-413). 


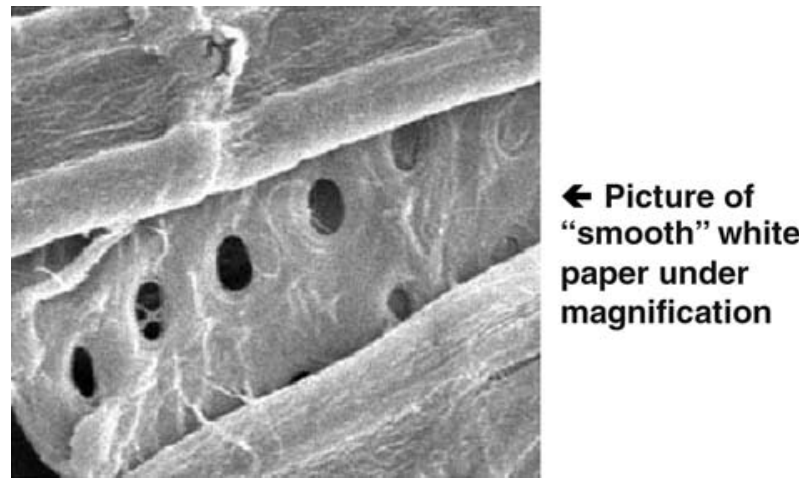

be one thing from this NS-empirical perspective. But when we look at the same paper with a microscope, S-empiricism enters the picture since I have more experiences compounding at that point and where I am no longer under the spell of merely blindly following my NS-empirical experience, and one sees that the paper is not smooth at all, but is composed of individual wood fibers, and thus in looking more close-up at the piece of paper, it does not appear to be one item: ${ }^{246}$

\footnotetext{
246 It is interesting to note that Avrum Stroll, in his book Surfaces, is aware of the conflict between empirical experiences of surfaces, depending on how close up or far back the observer is when looking at the surface, wherein, it would seem that the perspective creates the experience so the surface, not the surface itself creating it. Stroll first tells us that "marbles are good examples of things that are normally said to have surfaces." (1988 15) Then later Stroll tells us that
}

We do not speak about the surface of the sun. It is said that the sun's temperature at the surface is such and such; that giant gas tendrils stretch up from its surface and extend many miles above it; and that it is possible to calculate the distance from its center to its surface. We can say some of these things about marbles, too- that their temperature at the surface is such and such, for example. But there are some things we can say about marbles that we don't, perhaps can't, say about the sun. Is the surface of the sun pitted, blemished, smooth, rough, scratched, bruised, damaged, chipped? If we can't say these things, why not? Perhaps it is because the sun is not solid or even liquid...(1988, 28)

I am not sure why Stroll considers the smooth view of the surface to be in any way the same entity as the jagged surface. I am not sure how to avoid the conclusion here that these are experiences from differing perspectives (one closer-up than the other), and thus the experiencing creates the surface appearances. Stroll even maintains on page 15 that "[ $\mathrm{t}]$ he surfaces of marbles can be described as rough, smooth, slippery, chipped, sticky, blemished, pitted, or damaged...' If we take Stroll at his word, and if we believe that he wants to consider the surface of a marble to be a single entity, not eliminativistically reducible to a set of parts, then the surface of a marble, call it S, can described by the statement $\mathrm{M}^{1}$ :

$\mathrm{M}_{1}: \mathrm{S}$ is both rough and smooth at present $\mathrm{p}$.

If we take the word "smooth" to be synonymous with "rough", then Stroll's position that $\mathrm{M}_{1}$ can be both rough and smooth can be rewritten as $\mathrm{M}_{2}$ :

$\mathrm{M}_{2}$ : S can be both rough and not rough at present $\mathrm{p}$.

This is a contradiction, and would seem to merely imply what I am arguing in this article: a surface cannot be continuous (DIV2 perspective) and non-existence (DIV1 perspective) at the same time. 
If there was a person who had never seen a piece of paper before, and if I showed the microscopic picture of the paper to them and told them that this was a picture of an object that is smooth, continuous (unbroken, with no gaps between the parts), and white, I may be accused of hallucinating, and/or of not understanding language.

When I was a child, I wondered why when I looked at the hands of the clock I could not see them moving, only to see shortly after that they had moved. It appeared to me that the hands were still when I looked at them, and between long-enough times of looking at the clock hands, when I was looking away from them, they "jumped" to a new position. But my parents told that there is no such jumping. I was told that, surprisingly, the hands of the clock were moving-contrary to what my empirical experience told me-I just could not see them moving. In other words, I was told that I should not trust my empirical experience. Also when I was young, I was told that the earth was not flat, but rather was a giant ball, even though the earth appeared to me to be flat, other than minor inconsistencies such as mountains and hills. This also conflicted with my empirical experience, but I was told by parents and teachers to ignore my empirical experience. The sun appeared to me to be very much like the clock hands: the sun would not be seen to be moving at any instant, but over time it could be seen to have moved. So I imagined that, like the clock hands, the sun moved slowly and my senses could not detect the motion. This seemed satisfactory to me. But I was eventually told that the sun was not moving, but rather it was the earth that was moving, even though I surely could sense throughout the day that the sun was moving across the sky, just like I could sense that the clock hands were moving, and I could not detect the earth to be moving. I was told, again, that my sense information was wrong. Other examples like this which illustrate the fallibility of sense perception and of intuitive pre-philosophical analysis that is associated with NS-empiricism, are not difficult to find. Examples such as these and the many other ones I have given make it clear that, although empirical experience is the starting point of science and philosophy, under the more refined S-empiricism, there is understanding that blind faith in any sense experience (which is the hallmark of NS-empiricism) is not a careful sort of empiricism.

Example after example, I was told to ignore the inaccuracies of my senses. I grew up and got used to it, and then started to read philosophy, only to see philosophers surprisingly basing theories on the reliability of sense information. It seemed to me that humans forget about the inaccuracies of the senses as they grew up. When I arrived in college, I took classes in chemistry and physics, where I studied reality at the microscopic scale, at the microbiological level and smaller. If this was not enough to inform me of the truly staggering way my senses deceive me, I also studied the particles and forces of physics, and I was just told that they compose the reality we perceive; there was virtually no discussion of how these particles and forces, which, as we saw in above sections, did not at all appear to be such that they could compose the world I experienced in my daily life. Ford writes: 
When we look at the smallest specks of space and the tiniest ticks of time, we see what can only be called fireworks. Myriad new particles pop into existence, some long-lived, most short-lived,... and each capable of being destroyed as well as created. ${ }^{247}$

My point in bringing up these issues is to show that Markosian's error is merely the standard position among humans.

\section{Quantum consciousness, selves, ordinary experiencing}

\subsection{Overview: selves and experience}

As I have discussed, many readers may find mereological nihilism to have serious problems since it may not be currently known how consciousness (either DIV1 or DIV2 consciousness) can exist in a mereological nihilist reality, or since mereological nihilism may seem to imply that people do not exist. For example, Hudson appears to hold something along these lines, as seen in a passage of his that is about persons, and where he is talking about mereological nihilism and consciousness:

On this view the only material objects that exist are subatomic particles... To be sure, many philosophers have advocated the view that human persons are simples-immaterial ones-but no philosopher has seriously maintained (or should seriously maintain) that we are material subatomic particles such as bosons or classons or leptons. Even Roderick Chisholm who... suggested that human persons were smallish incorruptible material objects located somewhere in the brain, never went so far as to insist that we were material simples. $^{248}$

In the last section, we saw that this was analogous to asserting that, to give a third example, since there is not a clear definition of "life" that biologists agree on, then this is for some reason only a problem for small groups of biologists, such as exobiologists, or perhaps those that study parasite rex, ${ }^{249}$ which, although respectable areas of philosophy with a long tradition, are small and/or fringe areas of biology, and the "life" definition problem is for some reason not than being a problem for all of biology and philosophy of biology. Mereological nihilism does not necessarily lead to the conclusion that persons or consciousness do not exist. Mereological nihilism merely shows that consciousness and personhood cannot be composite entities. They are not the spatially extended physical human body, or a spatially extended physical brain-part-since the body and brain are composites and thus do not exist.

The inability to explain consciousness is not just a problem for mereological nihilism, but it is a problem for all of philosophy, and for physics, chemistry, biology, psychology, and neuroscience, since none of these theories are getting at what conscious experiencing is, or getting at what it is that explains what our

\footnotetext{
${ }^{247}$ Ford $(2004,2)$.

248 Hudson (2001b, 81-82).

${ }^{249}$ See Parasite Rex, by Carl Zimmer. 2000. Parasite Rex: Inside the Bizarre World of Nature's Most Dangerous Creatures. New York: Touchstone.
}

Springer 
"Technicolor phenomenology" (McGinn's terminology). It seems to be a basic quality of the philosophies of mind is that they really do not get at the explanation of the mental. ${ }^{250}$ Others have noticed this also. Chalmers writes: "Consciousness poses the most baffling problems in the science of the mind. There is nothing that we know more intimately than conscious experience, but there is nothing harder to explain." ${ }^{251}$ (A passage I will give from McGinn declared this same position in the last section.) The many substance and bundle theorists, ${ }^{252}$ for example, who are opponents of mereological nihilism and who are opponents the strictly empirical and scientific approach to the study of reality, do no better job than the mereological nihilists at explaining consciousness. ${ }^{253}$

In this section, I will give a general discussion of the only two possibilities I see for how consciousness can exist in a mereological nihilist reality. These positions depend on two positions that can be taken fore how one believes what atoms have to do with consciousness:

(i) it is believed that quantum abstract atoms cannot be conscious.

(ii) it is believed that quantum abstract atoms can be conscious, and

First I will discuss (i), which is a dualistic thesis (Sect. 9.2), and then I will discuss (ii), which is a monistic thesis (Sect. 9.3). The dualist position involves what I will call the McGinn mind, for reasons I will discuss. And the monistic position is a variety of what can be considered panpsychistic quantum consciousness. It should be admitted that each of these theories are unsatisfactory-at least from their current level of development, and unless a further advancement occurs. As for the unsatisfactoriness of the dualist position, dualism is currently largely rejected in Western philosophy for reasons that have been widely discussed. ${ }^{254}$ As for the unsatisfactoriness of the monistic position, it rest on the idea that partless unstructured point-particles that do little more than flash in and out of existence can give rise to consciousness. Needless

\footnotetext{
${ }^{250}$ I am reminded of an interesting quote here, which seems to perfectly illustrate the problem of the mind I am discussing: "The mind is a strange and wonderful thing. I'm not sure it will ever be able to figure itself out-everything else maybe, from the atoms to the universe, everything but itself." From Invasion of the Body Snatchers (1956 version).

251 Chalmers (1995, 200).

252 This is the group of metaphysical realist theorists who explain reality in terms of the way attributes of things (which are reducible to surfaces and colors of DIV2 experiencing) conglomerate and connect to give rise to objects of DIV2 experiencing. It seems this group of realists comprises the majority of American philosophers today. See Grupp (2006a, b, c) for problems with the account of reality this group presents.

${ }^{253}$ I have seen these critics who are bundle and substance theorists believe that they are describing consciousness by merely labeling brains as "having mental properties," as if that has explanatory value for the problem and mystery of consciousness. I am not sure how that is, in fact, not much more than a sentence that does very little to help understand anything at all, and which is really no more than the common sense view that is learned in elementary school when the teacher first-grade teacher teaches the students that "it is your brains that you use when you are thinking." The mereological nihilist also could merely label an atom of a nihilistic quantum abstract atomic reality as having mental properties if she/he so desired. This would produce the same level of explanation as maintaining that a set of non-mental on-off (firing-not-firing) neuron cells produce mental properties. In either case, explanation is not given as to how mental properties come out of the non-mental base material (neurons for the typical philosopher of mind, and atom(s) for the mereological nihilist).

${ }^{254}$ Blackmore $(2004,13)$.
} 
to say, it is not clear how this can be. ${ }^{255}$ But as unsatisfactory as these options may seem, they are no worse in explanatory value than the currently philosophies of mind we have been offered by philosophers in any other area of philosophy. In fact, I will discuss the problems of consciousness that encounter mereological nihilism are virtually the same problems that non-nihilistic philosophers run up against, and nonnihilistic philosophers are in the very same predicament as the nihilistic philosopher. The basic problems involve the idea that non-mental items (atoms, neurons, etc.) do not seem to be able to do the job of giving rise to our Technicolour phenomenology.

\subsection{Option 1: McGinn minds: non-physical minds that are ineffable}

I will first discuss the idea that quantum abstract atoms cannot be considered to be conscious. Consider the following two arguments, which give an idea of the difficulty involved with the question of consciousness and mental experience in the mereological nihilist reality:

A. Consciousness exists.

B. There is no brain or body. (This follows from the sections above.)

C. Therefore, consciousness is not the brain and/or body activity.

As for premise A, many philosophers claim it is self-evidently true that there are experiences, mental states, phenomenal states, etc. If it is, then DIV2 experiencing would be consciousness that does not represent reality accurately, but it nevertheless exists. Now consider another argument:

D. Consciousness exists.

E. It does not seem that quantum abstract atoms can be consciousness nor experiences.

F. Therefore, it does not seem that consciousness is made up of quantum abstract atoms.

Putting $\mathrm{C}$ and $\mathrm{F}$ together implies that if there is consciousness, it is not nervous system activity, and it is not atomic activity, and thus there can only be a dualism in mereological nihilism: in mereological nihilist reality, there are two kinds of items that exist: unconnected and unattached quantum abstract atoms, and phenomenal states of consciousness. Quantum abstract atoms can be studied through phenomenal states of consciousness, but phenomenal states of consciousness study themselves. This appears to be something like the position that Colin McGinn has espoused in his "cognitive closure" position:

\footnotetext{
255 Also, I imagine that many philosophers will consider the monistic position to be questionable since they will be associate it with a type of mysticism since it involves panpsychistic substratum of energy (particles). But I see this as perhaps incorrect to associate this with mysticism, since Western mysticism appears to involve oneness of some sort with God, and Hindu and Sufi mysticism also appears to involves some sort of bond with God. Nowhere in this article does the metaphysical concept of God fit in, whether Hindu, Sufi, or Christian, and thus I am not sure how quantum mereological nihilism can be associated with mysticism in any way. Buddhistic accounts from India are astonishingly close to the quantum panpsychistic idea. But I am not sure how and why those accounts can be considered mystical, since Buddhists from India are infamously atheistic, and since that tradition involves rigorous philosophical analysis to get to theory Buddhist atomism. So I do not know how Buddhism from India can be considered mystical.
} 
The problem arises, I want to suggest, because we are cut off by our very cognitive constitution from achieving a conception of that natural property of the brain (or of consciousness) that accounts for the psychophysical link. This is a kind of causal nexus that we are precluded from every understanding, given the way we have to form our concepts and develop our theories...

Let me introduce the idea of cognitive closure. A type of mind $\mathrm{M}$ is cognitively close with respect to a property $\mathrm{P}$ (or theory $\mathrm{T}$ ) if and only if the concept-forming procedures at Ms' disposal cannot extend to a grasp of $\mathrm{P}$ (or an understanding of T). Conceiving minds come in different kinds, equipped with varying powers and limitations, biases and blindspots, so that properties (or theories) may be accessible to some minds but not to others. What is closed to the mind of rat may be open to the mind of a monkey, and what is open to us may be closed to the monkey... This is particularly clear for perceptual faculties, of course: perceptual closure is hardly to be denied... [N]o species can perceive every property things may instantiate (without artificial instrumentation anyway). But such closure does not reflect adversely on the reality of the properties that lie outside the representational capacities in question; a property is no less real for not being reachable for a certain kind of perceiving and conceive mind. ${ }^{256}$

If conscious experiencing is not particles, and if mind and conscious experiencing exists, it seems that the following conclusion is a viable option for the mereological nihilist: consciousness is some sort of "exotic stuff:" it is not the same type of stuff as quantum abstract atoms and not made up of quantum abstract atoms. It must be admitted that this is a dubious suggestion, since, due to the reasoning in the sections above, McGinn minds must be partless point-atoms, and I do not understand how they could be described in any way as being different from quantum abstract atoms. But I will assume that somehow they can be, in order to further discuss this dualist option more in this subsection, and I will the account of their being identical to the quantum abstract atoms in the next section.

According to the dualist option, consciousness would be an item that is an entirely inconceivable "stuff" that cannot be labeled matter, particles, or quantum abstract atoms. With quantum abstract atoms, we can at least maintain that they are as we have described them in this article (immaterial, partless, etc.), but the McGinn minds, we could do little more than generate a statement the following in describing them: they are partless, inconceivable, and ineffable.

On this account, I see no reason why I should avoid the suggestion that somehow McGinn minds might be able to directly apprehend quantum abstract atomic reality: if a McGinn mind ever were to experience something real, it can directly experience quantum abstract atoms, giving rise to a DIVI I-empirical experience. Surely this may sound inane to many philosophers of mind, since I am suggesting here both that a consciousness is not a brain activity, and I don't see a reason why that non-nervous system consciousness cannot directly (not with the aid of the machinery and apparatuses of physics: supercolliders, atoms smashers, mathematical description, etc.) experience quantum abstract atoms. So McGinn minds in a mereological nihilist reality are not so controversial, and they involve some of the primary tenets of philosophy of mind. Also, other philosophers of mind do not have an account for consciousness. The idea that McGinn minds might be able to directly

256 Ibid., 2-3. 
apprehend quantum abstract atoms would make the atoms directly empirical. Above I referred to empirical experience as "ordinary empirical experience." Witnessing quantum abstract atoms directly might need to be called something else, such as nirvana, as Buddhist philosophers may choose. Perhaps our DIV2 oriented minds are often susceptible to interstices of DIV1 experience.

The nihilists, Rosen and Dorr, appear to come to a very different conclusion: in a passage where they discuss the self (so they may or may not be referring to merely conscious experiencing, as I have above, and which does not necessarily require the existence of a self or persisting self): rather than maintaining that there is a self, they conclude that if premise E is true, then the self would not exist: "Since you are not one of these particles [quantum abstract atoms] and since there are no other candidates, the compositional nihilist maintains that strictly speaking, you do not exist." ${ }^{, 57}$ If Rosen and Dorr mean that self is consciousness, then there might not be a self and furthermore there might not be any consciousness. If they do, it seems they are merely assuming the negation of my premise D. But they do not provide an argument for this, and I therefore instead follow my immediate experience, maintaining that it necessarily exists. If it did not, right now I would be a thinker that was trying to theorize that there are no thinkers, but that is a contradiction. Premise D is an assumption, but it seems a good one due to the fact that, so far as anyone can tell, inner/subjective mental states, phenomenal states, etc. cannot be made of one atom, or multiple unattached/ unconnected atoms, and therefore are not made of atoms at all.

If mereological nihilism is correct, then brains do not exist, and consciousness is in no way a brain activity. I imagine many philosophers may find this a very strange position, but it is important to note that McGinn minds are very much like traditional dualist accounts. For example, McGinn minds involve a characteristic of the traditional and mainstream dualist philosophies of mind, where it is held the mental properties are not reducible to brains, and thereby are not brain activities. The idea that experience is not a brain activity, but is the activity of a brainless mind, if you will, has been held by some of the aforementioned philosophers who have been mereological nihilists or who hold positions very similar to mereological nihilism. For example, some accounts of the pre-classical Indian Buddhism and in Kantian transcendental idealism, there is found to be two sorts of "stuff" that composes reality: phenomenal states and atoms (in Kantian terminology, these would be called transcendental egos and thing-in-themselves). In a passage about Kant and Indian Buddhism, Stcherbatsky calls the Buddhist "double reality, the ultimate reality of things by themselves and the psychologically constructed reality (i.e., unreality) of empirical things." 258 The "double reality," to use Stcherbatsky's term, is empirical versus atomic reality, or unreal-

\footnotetext{
257 Rosen and Dorr (2002, 152).

258 Stcherbatsky, F. Th. 1962 (1930). Buddhist Logic. Volume 1. page 143. Stcherbatsky discusses this in a very interesting passage where he is comparing Buddhism to the philosophy of Immanuel Kant. Many have drawn similarities between some Europeans, such as Hume and Kant, and the Indian Buddhists. But note that we bring up Kant only to discuss his dual reality, which is in agreement with this article. There is however an important difference to note between this Kantian metaphysics on the one hand and Buddhist atomism and this article on the other. Kant held that the real (things in themselves, which apparently are philosophic atoms) do not influence or causally impact the mind in any way. This is an assumption that I reject, and I hold the opposite assumption: mind is impacted by atoms, but the mind misinterprets the atoms as being extended surfaces and color patches of the empirical.
} 
empirical versus actual-atomic reality: the conceptual versus the real. In this section, I will show that this double reality means that, at the present moment, our best alterative in the philosophy of mind is to maintain that experience is "made of" some sort of non-atomic "exotic stuff" which are do not have a theory for, and may never have a theory for.

\subsection{Option 2: Quantum panpsychism}

As we saw, it is very questionable as to how McGinn minds, which must be nonphysical point atoms can be considered somehow a different kind of stuff than quantum abstract atoms. I will next explore the idea that they are not different. This leads to a philosophy that consists of a mixture of panpsychism and perhaps a theory of quantum consciousness.

Theories of quantum consciousness involve the theory that consciousness has to some degree something to do not just with neurons, but also with the quantum effects of reality. But according to mereological nihilism, there are no neurons, and consciousness has only to do with quantum abstract atoms. Since there are no brains, no neurons, no immaterial mental substances-since each of these are mereological wholes, which do not exist-and since there are only quantum abstract atoms, then consciousness can only be identical to, the quantum abstract atoms themselves. I can think of no other option for consciousness other than this if mereological nihilism is correct and the dualism of the previous section is not correct. If that is the only option, then this is a theory of quantum consciousness, ${ }^{259}$ and it is also a theory that appears to be panpsychistic: since quantum abstract atoms are structureless (point-sized) items with no means of being distinguished from one another, and if consciousness has something to do with one or a few of the atoms, then it can only have to do with all of them.

Since this thesis of panpsychism being suggested here in this part of this article involves a monistic universe-less reality that is immaterial and conscious, some may wish to assert that it is also spiritual also. The model of reality being described in this article and especially in this section, has many similarities to, for example, the logical epistemology of the Buddhist Dharmakirti (India, 7th century), and perhaps to much of the Buddhism of India in general-which also often takes the form of a theory of quantum consciousness. But nothing in the above argumentation would lead to the inference that in addition to being all of

\footnotetext{
259 It is a quantum consciousness theory in that the only real items that consciousness can be aware of, and/or be caused by, are Buddhist atoms (quantum abstract atoms). This is called the standard interpretation in Indian Buddhism. In his book on Dharmakīrti, Dreyfus writes:
}

According to the Sautrāntika explanation, only infinitesimal atoms and moments of consciousness are real. Everything else, such as a shape or a color, is real only inasmuch as it is taken as an object of conventional practice. This view is not unlike Wilfrid Sellar's claim that objects such as tables, ice cubes, and colors do not really exist... Our commonsense notions of such objects are false but cognitively useful......[A]lthough Dharmakīrti never provides a detailed statement of this ontology, we could expect him to follow th[e] Sautrātika view. Several traditional and modern scholars have explained Dharmakīrti in this way, emphasizing that in his system reality is reducible to partless atoms interacting with moments of consciousness... This... explains our perceptions of extended objects. In reality, there is no extension but just the causal interaction of infinitesimal atoms with partless moments of consciousness. I call this interpretation of Dharmakirti's ontology the standard interpretation. (Dreyfus 1997, 85) 
these, this reality is therefore also spiritual. The spiritual usually has to do with something metaphysical, and as discussed above this article is largely about eradication of the metaphysical.

I am not the only one to notice stark similarities between science and Buddhism with respect to the study of consciousness. In her recent book, Consciousness, Blackmore reserves the last chapter for an analysis of Buddhism and consciousness, where she comes to the following point, which is in precise agreement with my findings of this article:

One point that Buddhism and psychology both make is that our experience is, in some sense, illusory. Since an illusion is not something that does not exist, but something that is not what it seems, this leaves plenty of room for different interpretations. In science we have already met the idea that the visual world might be a grand illusion, that the stream of consciousness might be illusory, as might both the self and free will. We can now see that there are distinct similarities between the illusions discussed in science and in Buddhism. ${ }^{260}$

\section{Conclusion}

The attacks against material composition I have discussed in this article reveal fatal problems for composition to which I see no solution. This article also establishes mereological nihilism as a leading interpretation of quantum physics-and perhaps the most appealing to the anti-metaphysical crowd. My intention in this article has been to bring these issues to the attention of philosophers and physicists since, oddly, there have been so few cohorts of quantum mereological nihilism. If mereological nihilism is the correct interpretation of quantum reality, then as I see it, there would be three mysteries surrounding it:

1. How can there be consciousness in a mereological nihilist reality?

2. Why and/or how to particles emerge into existence, seemingly "from nothing"?

3. Why do humans see patterns (interference and wave patterns, etc.) in the particle flashings (such as in the two slit experiment)?

If the reasoning I have presented in this article is correct, matter does not exist, reality is immaterial energy, only quantum atoms exist, which are immaterial points of energy that "vibrate" in and out of existence. It seems to me that this description of reality is so similar to some of the Buddhists of pre-classical India that this article could (and perhaps should) lead to a revolutionary upwelling of interest in Buddhist psychology, positivism (in Comte's sense of the word) and empiricism, pragmatic philosophy, all in combination with a non-metaphysical interpretation of quantum physics. To a huge extent, a Baconian-level trust in science is lacking in the denizens the contemporary world. Such a trust in them would lead to affirming revolution. The power and enlightenment that can be found in science is largely absent from, and unknown to, the denizens of the world. The denizens of the world are often taught how to perform at unfulfilling occupations that give little pay, long hours, and shallow existence, but they are nearly never taught how to live-what Thoreau called

\footnotetext{
${ }^{260}$ Blackmore $(2004,411)$.

型 Springer
} 
"the art of living." Buddhism is the pinnacle of study of the inner (mental) world, ${ }^{261}$ and quantum physics is the pinnacle of science. The unification of these represents the pinnacle of the study of reality as-a-whole, which is the most worthwhile study. The world today is replete with citizens who are in need of this study. They are in need of a proper psychology to help them get by rationally with their daily life lest ennui and violence cripple their lives. The citizens of the world also are in drastic need to know that scientific thinking is not something special that is reserved for laboratories and universities, but, rather, it must be the essence of one's daily life, needed in order to live properly: the proper life is the one that studies, both the inner and the outer, and, ultimately, the blurred amalgam of the two. The improper life, the life that consists of confusion and lostness, is the life that is devoted to distraction only. (It is improper because it does not contribute to humanistic pursuits, but rather only in only contributes to the pursuits and dreams of those who have enslaved that mind and life.) To a large extent, the citizens of today's world, it appears safe to assert, are lost in metaphysics, entertainment, uncritical thinking, guilt, various kinds of poverty, and an utter lack of feeling of profundity and energy. The positivistic amalgam of quantum physics and Buddhism - which is the truest sense of what quantum mereological nihilism involves-offers an antidote to the this pervasive and contemporary human state of being unaware, uninformed, unintelligent, stolid, confused, and without an inner fire that is burning. Mereological nihilism evokes a release from concern over the sea of trivial matters that tie one to shallow existence, and instead leads to a focus only on the immaterial energy that is reality, and which is all human subjective vitalism-which is the essence of Buddhist ethics. ${ }^{262}$

\section{References}

Albert DZ (1992) Quantum mechanics and experience. Harvard University Press, Cambridge Blackmore S (2004) Consciousness. Oxford University Press, New York

Brower J (2002) Relations without polyadic properties: Albert the great on the nature and ontological status of relations. Arch Geschichte der Philos 83(2001):225-257

Casati R, Varzi A (1999). Parts and places. MIT Press, Cambridge

Chalmers D (1995) Facing up to the Problem of Consciousness. J Consciousness Stud 3(1):200-219

Chisholm R (1989) On metaphysics. University of Minnesota Press, Minneapolis

Cohn A, Varzi A (2003) Mereotopological connection. J Philos Logic 32:357-390

Davies P (1984) Superforce. Touchstone, New York

Davis I (2006) The notion of haecceitism: are haecceities able to account for individuation? Midsouth Philosophy Conference. Memphis Tennessee, February 25, 2006

${ }^{261}$ From his classic text, Recognizing Reality: Dharmakìrti's Philosophy and its Tibetan Interpretations, Dreyfus discusses this:

Buddhism teaches that human beings can liberate themselves from suffering through a correct understanding of reality. We usually understand reality through perception and from wrong views, such as the substantial existence of persons, on the basis of these experiences. So the first step in a Buddhist liberative strategy is to gain a clear understanding of experiences. This is achieved by developing mindfulness toward the four kinds of objects: body, feeling, consciousness, and mental factors. (Dreyfus 1997, 98.)

This seems, to some degree, to possibly reflect the Socrartic maxim, "the unexamined life is not worth living."

${ }^{262}$ I am grateful to two anonymous referee at Axiomathes for helpful comments. I am grateful to Quentin Smith for discussing Sect. 4.2.3 with me. I am grateful to Professor John Morris of Indiana University Northwest's Physics Department for discussing many ideas in physics with me. 
Davies PCW, Brown JR (eds) (1986) The ghost in the atom. Cambridge University Press, Cambridge Davies PCW, Brown JR (eds) 1992 (1988) Superstrings: a theory of everything? Cambridge University Press, Cambridge

Davies P, Gribbin J (1992) The matter myth. Touchstone, New York

Dorr CS (2002) The simplicity of everything. Dissertation, Princeton University

Dreyfus GBJ (1997) Recognizing reality: Dharmakīti's philosophy and its Tibetan interpretations. State University of New York Press, Albany

Edgar W (1979) Locations. Can J Philos IX(2):323-333

Einstein A (1950) The meaning of relativity. Princeton University Press, Princeton

Elder CL (2003) Destruction, alteration, simples, and worldstuff. The Philosophical Quarterly 53(210):24-38

Faye J. (2002) Copenhagen interpretation of quantum mechanics. In: Zalta EN (ed) The Stanford Encyclopedia of Philosophy (Summer 2002 Edition), URL $=<$ http://plato.stanford.edu/archives/sum2002/entries/qm-copenhagen/ >

Feldman J (2005) Vasubandhu's illusion argument and the parasitism of illusion upon veridical experience. Philosophy East and West 55(4):529-541

Ford KW (2004) The quantum world. Harvard University Press, Cambridge

French S (2006) Identity and individuality in quantum theory. In: Edward N. Zalta (ed) The Stanford Encyclopedia of Philosophy (Spring 2006 Edition), URL = http://plato.stanford.edu/archives/ spr2006/entries/qt-idind/

Gibbins P (1987) Particles and paradoxes: the limits of quantum logic. Cambridge University Press, New York

Greene B (1999) The elegant universe. W.W. Norton and Co., New York

Greene B (2004) The fabric of the cosmos. Vintage, New York (a division of Random House)

Griffiths DJ (1995) An introduction to quantum mechanics, 1st edn. Prentice Hall, Upper Saddle River

Gribbin J (1998) Q is for Quantum: an Encyclopedia of particle physics. Touchstone, New York

Grünbam A (1952) A consistent conception of the extended linear continuum as an aggregate of unextended elements. Philos Sci XIX:288-306

Grünbaum A (1955) Modern science and refutation of the paradoxes of Zeno. In Salmon W (ed) Zeno's paradoxes 2001. Hackett, Indianapolis

Grünbam A (1967) Zeno's metrical paradox of extension. In Salmon W (ed) Zeno's paradoxes, 2001. Hackett, Indianapolis

Grupp J (2003) The impossibility of an exemplification tie between particulars and universals. Metaphys: The Int J Ontol Metaphys 4(1):27-38 (This paper can be read at www.abstractatom.com.)

Grupp J (2004a) Problems with the platonist exemplification tie between located entities and an unlocated entity. Dialogue: Can Philos Rev XLIII:491-498 (This paper can be read at www.abstractatom.com.)

Grupp J (2004b) Compresence is a bundle: a problem for the bundle theory of objects. Metaphy: The Int J Ontol Metaphys 5(2):63-72

Grupp J (2005a) "The R-theory of time, or replacement presentism: the Buddhist philosophy of time. The Indian Int J Buddhist Stud (IIJBS) 6:51-122

Grupp J (2005b) The impossibility of relations between non-collocated spatial objects and nonidentical topological spaces. Axiomathes 15(1):85-141(57). (This paper can be read at www.abstractatom.com.)

Grupp J (2005c) The impossibility of temporal relations between non-identical times: new arguments for presentism. Disputatio: Int J Philos, May (This paper can be read at www.abstractatom.com.)

Grupp J (2005d) Western analytic metaphysics reduces to a philosophy of Brahman, part 1. J Indian Council Philos Res (JICPR). XXI(2) (In press.) (This paper can be read at www.abstractatom.com.)

Grupp J (2006a) Blob theory. Sorites 17 (Forthcoming). (This paper can be read at www.abstractatom.com.)

Grupp J (2006b) Western analytic metaphysics reduces to a philosophy of Brahman, part 2. J Indian Council Philos Res (JICPR). (This paper can be read at www.abstractatom.com.)

Grupp J (2006c) God's spatial unlocatedness prevents him from being the creator of the universe. A New argument for the nonexistence of god. Sophia: Int J Philos Religion, Metaphys Theol Ethics 45(1) (This paper can be read at www.abstractatom.com.) 
Hawley K (2004) Temporal parts. In: Zalta EN (ed) The Stanford Encyclopedia of Philosophy (Winter 2004 Edition), URL $=<$ http://plato.stanford.edu/archives/win2004/entries/temporalparts/ >

Heisenberg W 1999 (1958). Physics and philosophy: the revolution in modern science. Prometheus Books, Amherst New York

Herbert N (1985) Quantum reality. Anchor, New York

Herbert N (1989) Faster than light. Plume, New York

Hoffman J, Rosenkrantz G (1997) Substance: its nature and existence. Routledge, London

Horgan T (1993) On what there isn't. Philos Phenemonel Res LII(3):693-700

Hossack K (2000) Plurals and complexes. Brit J Philos Sci 51:411-443

Hudson H (2001a) Touching. Philos Perspect 15:119-128

Hudson H (2001b) A materialist metaphysics of the human person. Cornell University Press, Ithaca Ismael J (2004) Quantum mechanics. In: Zalta EN (ed) The Stanford Encyclopedia of Philosophy (Fall 2004 Edition), URL $=<$ http://plato.stanford.edu/archives/fall2004/entries/qm/ >

James W 1996 (1912) Radical empiricism. Bison Books, New York

James W (1992) Reflex action and theism. In: Myers GE (ed) William James: writings: 1878-1899. The Library of America, New York, pp 540-565

Jammer M 1993 (1954) Concepts of space. Dover, New York

Jammer M 1999 (1957) Concepts of force. Dover, New York

JHA VN (1990) The philosophy of relations (Containing the Sanskrit text and English translation of Dharmakīrti's Sambandha-parìksā Prabhācandra's Commentary), Sri Satguru Publications, Dehli

Jones T (2004) Reductionism and antireductionism: rights and wrongs. Metaphilosophy 35(5): 614-647

Kane G (2000) Supersymmetry. Perseus Publishing, Cambridge

Kant I (2004) The metaphysical foundations of natural science. Translated and Edited by Michael Friedman. Cambridge University press, Cambridge

Kline DA, Matheson CA (1987) The logical impossibility of collision. Philosophy 62:509-515

Lange M (2002) An introduction to the philosophy of physics. Blackwell, Malden

Lockwood M (1989). Mind, brain, and the quantum: the compound "I". Blackwell, New York

Loux M (1998). Metaphysics: a contemporary introduction. Routledge, New York

Loux M (2001) Metaphysics: Contemporary readings. Routledge, New York.

Lowe EJ (2002) A survey of metaphysics. Oxford University Press, New York

Markosian N (1998a) Simples. Aust J Philos 76:213-226

Markosian N (1998b) Brutal composition. Philos Stud 92:211-249

Matthieu R, Thuan TX (2001) The quantum and the lotus. Three Rivers Press, New York

Maudlin T (2001). Quantum non-locality and relativity. Blackwell, Malden

McGinn C (1991). The problem of consciousness. Blackwell, Cambridge

Merricks T (2001). Objects and persons. Oxford University Press, New York

Moreland JP (2003) Bare particulars and individuation: a reply to mertz. Aust J Philos 81(1):1-13

Murdoch D (1987). Niels Bohr's philosophy of physics. Cambridge University Press, New York

Nadeau R, Kafatos M (1999). The non-local universe. Oxford University Press, New York

Nerlich G (1994) The shape of space, 2nd edn. Cambridge University Press, Cambridge

Omnès R (1999a) Understanding quantum mechanics. Princeton University Press, Princeton

Omnès R (1999b) Quantum philosophy. Princeton University Press, Princeton

Petitot J, Smith B (1997) Physics and the phenomenal world. In Poli R, Simons PM (eds) Formal ontology. Kluwer, Dordrecht/Boston/London pp 233-253

Pratt I, Schoop D (1998) A complete axiom system for polygonal mereotopology of the real plane. J Philos Logic 27:621-658

Pyle A (1995). Atomism and its critics. Thoemmes Press, Bristol New York

Rae M (1997). Material constitution: a reader. Rowman and Littlefield Publishers, Lanham

Richard M, Xuan Thuan T (2001) The quantum and the lotus. Three Rivers Press, New York

Rosen G, Dorr C (2002). Composition as fiction. In: Gale R (ed) The Blackwell guide to metaphysics. Blackwell, Malden, pp 151-174

Schaffer J (2003). Is there a fundamental level? Noûs 37(3):498-517

Sider T (1993) Van Inwagen and the possibility of gunk. Analysis 53(4):285-289

Sklar L (1977) Space, time, and spacetime. University of California Press, Berkeley

Simons P (1987) Parts: a study in ontology. Oxford University Press, Oxford

Smith Q (1984) The conceptualist argument for god's existence. Faith Philos 11(1):38-49

Smith B (1996) Mereotopology: a theory of parts and boundaries. Data Knowledge Eng 20:287-303 
Smith B (1997) Boundaries: an essay in mereotopology. In Hahn L (ed) The philosophy of Roderick Chisholm (Library of Living Philosophers). Open Court, LaSalle, pp 534-561

Sorenson RA (1998) Sharp boundaries for blobs. Philos Stud 91:275-295

Stcherbatsky FTh 1962 (1930) Buddhist logic, Vol. 1. Dover, New York

Strawson G (1994) Mental reality. MIT Press, Cambridge

Stroll A (1988) Surfaces. University of Minnesota Press, Minneapolis

Swinburne R (1995) Thisness. Aust J Philos 73:389-400

van Inwagen P (1993) Précis of material beings. Philos Phenomenol Res LIII(3):683-686

van Inwagen P (1990) Material Beings. Cornell University Press, Ithaca

Watson A (2004) The quantum quark. Cambridge University Press, New York

Wiggins D (1997) On being in the same place at the same time. In Rae M. (ed) Material constitution: a reader. Rowman and Littlefield Publishers, Lanham, pp 3-9

Wolfe D (2001) Tales from the underground: a natural history of subterranean life. Perseus Publishing, Cambridge

Zimmerman D (1996a) Could extended objects be made out of simple parts? an argument for 'atomless gunk'. Philos Phenomenol Res LVI(1):1-29

Zimmerman D (1996b) Indivisible parts and extended objects: some philosophical episodes from topology's prehistory. The Monist 79(1):148-180

Zimmerman DW (1998) Temporary intrinsics and presentism. In: van Inwagen P, Zimmerman D (eds) Metaphysics: the big questions. Blackwell, Oxford, pp 206-219 